\title{
Improved Melt Pool Monitoring Systems for Direct energy Deposition Processes
}

\section{Sampson, Robert J.}

How to cite:

Sampson, Robert J. (2019) Improved Melt Pool Monitoring Systems for Direct energy Deposition Processes. Doctoral thesis, Swansea University.

http://cronfa.swan.ac.uk/Record/cronfa52474

Use policy:

This item is brought to you by Swansea University. Any person downloading material is agreeing to abide by the terms of the repository licence: copies of full text items may be used or reproduced in any format or medium, without prior permission for personal research or study, educational or non-commercial purposes only. The copyright for any work remains with the original author unless otherwise specified. The full-text must not be sold in any format or medium without the formal permission of the copyright holder. Permission for multiple reproductions should be obtained from the original author.

Authors are personally responsible for adhering to copyright and publisher restrictions when uploading content to the repository.

Please link to the metadata record in the Swansea University repository, Cronfa (link given in the citation reference above.)

http://www.swansea.ac.uk/library/researchsupport/ris-support/ 


\title{
Improved Melt Pool Monitoring Systems for Direct Energy Deposition Processes
}

\section{ROBERT JOHN SAMPSON}

\section{Submitted to Swansea University in} FULFILMENT OF THE REQUIREMENTS FOR THE Degree of Doctor of Philosophy

\author{
SWANSEA UNIVERSITY
}

2019 


\section{Declaration}

This work has not previously been accepted in substance for any degree and is not being concurrently submitted in candidature for any degree.

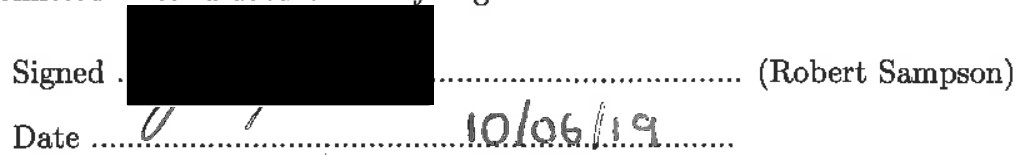

This thesis is the result of my own investigations, except where otherwise stated. Other sources are acknowledged by footnotes giving explicit references. A bibliography is appended.

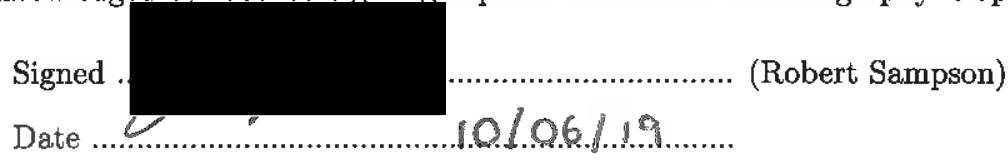

I hereby give consent for my thesis, if accepted, to be available for photocopying and for inter-library loan, and for the title and summary to be made available to outside organisations.

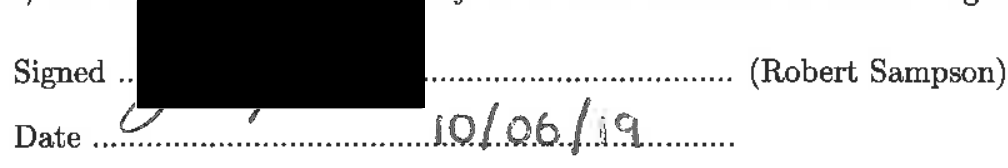




\section{Acknowledgements}

Thank you to both Swansea University and TWI for providing academic and industrial support for this research.

Thank you to Robert Lancaster, Mark Sutcliffe, David Caswell, Carl Hauser and Josh Barras. You have all provided me with valuable information throughout the years.

Finally a special thanks to my friends, family and partner that have constantly shown support throughout my life. Your love and affection provides the inspiration for this research. 


\section{Abstract}

Additive Manufacturing (AM) processes have previously benefited from the introduction of parameter monitoring systems, with melt pool monitoring majorly contributing to this field of research. Current melt pool monitoring systems are typically emissivity-based image processing techniques. Melt pool monitoring systems have successfully been used to grant advanced process understanding, develop control systems and improve deposition quality. By improving the optical acquisition techniques in melt pool monitoring systems, a more enhanced and clearer image of the melt pool has been created, which contains features that indicate the true edges. These new features have been used to compare the conventional emissivity-based image processing techniques with a newly developed image processing technique. Comparing both techniques with extracted frames has highlighted potential flaws in conventional image processing algorithms and improved the understanding of melt pool radiation mechanics and dynamics throughout direct energy deposition processes. The newly developed algorithm provided more accurate melt pool width calculations when compared with the emissivity-based edge detection technique and has been used to study changes in melt pool width with varying laser power settings. The new algorithm was used for subsequent parametric studies to understand the relationship between powder mass flow rate, path velocity and melt pool width. It was discovered that the relationship between these parameters and melt pool width was complex and further parametric studies using the optimised melt pool algorithm should be conducted. The new algorithm was successfully used to calculate melt pool widths for multiple materials without the need for emissivity values. Melt pool width calculations were performed on multiple materials after minor calibration procedures. 


\section{Acronyms}

AM Additive Manufacturing

AOI Area Of Interest

API Application Programming Interface

BoW Bag of Words

CCD Charged-Couple Device

CMOS Complementary Metal-Oxide Semiconductor

CNN Convolutional Neural Network

COM Centre Of Mass

CT Computed Tomography

DBN Deep Belief Networks

DED Direct Energy Deposition

DOF Depth Of Field

EBM Electron Beam Melting

FOV Field Of View

IR Infrared

LMD Laser Metal Deposition

LUT Laser Ultrasonic Testing

ML Machine Learning

MLP Multi-Layer Perception 
NDT Non-Destructive Testing

NIR Near-Infrared

PBF Powder-Bed Fusion

PMFR Powder Mass Flow Rate

ROI Region Of Interest

SIFT Scale Invariant Feature Transform

SL Stereolithography

SLM Selective Laser Melting

SLS Selective Laser Sintering

SVM Support Vector Machine

WAALM Wire and Arc Additive Layer Manufacturing 


\section{Contents}

$\begin{array}{lll}\text { Declaration } & \text { i }\end{array}$

$\begin{array}{ll}\text { Acknowledgements } & \text { ii }\end{array}$

$\begin{array}{lll}\text { Abstract } & \text { iii }\end{array}$

Acronyms $\quad$ iv

List of Figures $\quad$ xvii

List of Tables $\quad$ xviii

1 Introduction $\quad 1$

1.1 Motivation for Research . . . . . . . . . . . . . . . . . . . . . . . . 1

1.2 Principal Hypothesis . . . . . . . . . . . . . . . . . . . . 4

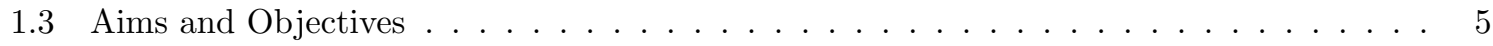

1.4 Research Methodology . . . . . . . . . . . . . . . . . . . . . 5

1.5 Summary of Contribution to Knowledge . . . . . . . . . . . . . . . . . . . 6

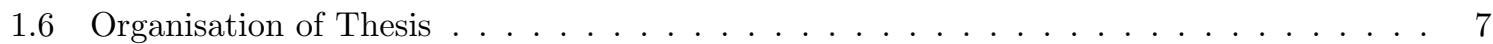

2 Review of Literature $\quad 9$

2.1 A Brief History of Additive Manufacturing . . . . . . . . . . . . . . . . . . . . . . 9

2.2 Additive Manufacturing Processes . . . . . . . . . . . . . . . . . . . . . 10

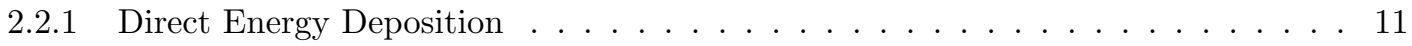

$2.2 .2 \quad$ Powder Bed Fusion . . . . . . . . . . . . . . . . . . . . . . . . . . . 14

2.3 Metal Powders in Additive Manufacturing . . . . . . . . . . . . . . . . . . 16

2.4 The Economics of Additive Manufacturing . . . . . . . . . . . . . . . . . . . . . 17

2.5 Parameter Monitoring in Additive Manufacturing . . . . . . . . . . . . . . . . . 18

2.5 .1 Pyrometry . . . . . . . . . . . . . . . . . . . . . 19 


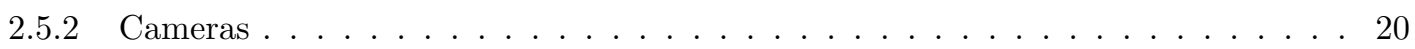

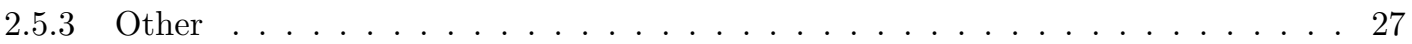

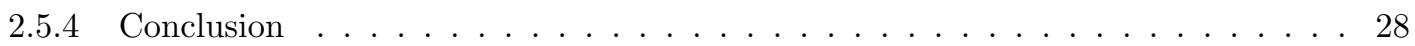

2.5.5 State of Knowledge and Literature Gaps . . . . . . . . . . . . . . . . 29

$2.5 .6 \quad$ Final Review . . . . . . . . . . . . . . . . . . . . . 30

3 Machine Vision in Laser Metal Deposition Processes 33

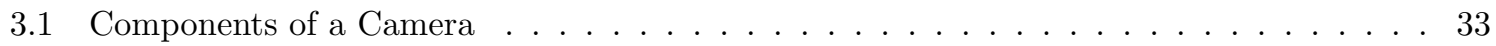

3.1 .1 Image sensors . . . . . . . . . . . . . . . . . . . . . 33

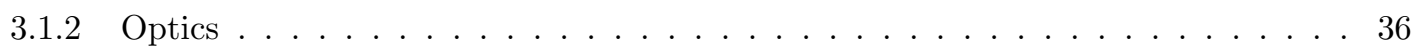

3.2 Camera Settings . . . . . . . . . . . . . . . . . . . . 37

3.2 .1 Shutter Mode . . . . . . . . . . . . . . . . . . . 37

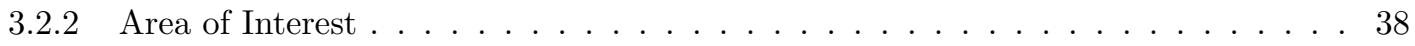

$3.2 .3 \quad$ Frame Rate . . . . . . . . . . . . . . . . . . . . . . . . . . 38

3.2 .4 Exposure Time . . . . . . . . . . . . . . . . . . . 38

3.3 The Directional Emittance Phenomena . . . . . . . . . . . . . . . . . . . . . 39

3.4 Experimental set-up for advanced melt pool imaging . . . . . . . . . . . . . . . 42

3.4 .1 Direct Energy Deposition Machine . . . . . . . . . . . . . . . . . . 42

3.4 .2 Camera . . . . . . . . . . . . . . . . . . . . . . 43

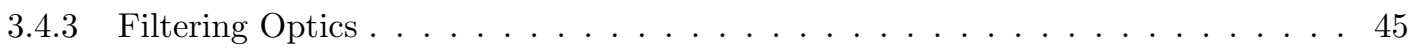

3.4 .4 Optics Installation and Imaging Parameters . . . . . . . . . . . . . 46

3.4.5 Measuring Powder Mass Flow Rate . . . . . . . . . . . . . . . . . . . 48

3.4 .6 Powder Material . . . . . . . . . . . . . . . . . . . 50

3.5 The Effects of Exposure Time on Melt Pool Imagery . . . . . . . . . . . . . . . 50

3.6 Preliminary Exposure Time Experiment . . . . . . . . . . . . . . . . . . . 54

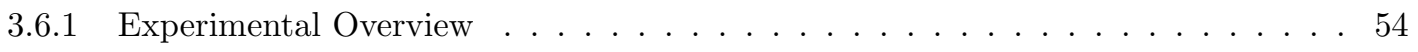

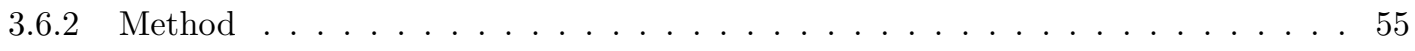

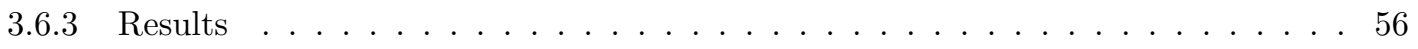




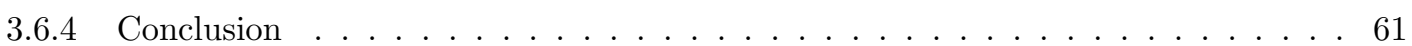

3.7 Adaptive Exposure Times for Varying Laser Power Settings . . . . . . . . . . . . . . . 62

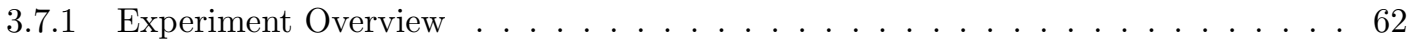

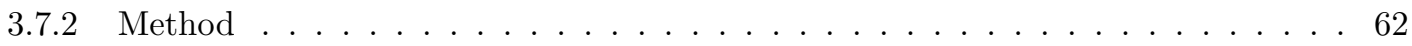

3.7.3 Experimental Results . . . . . . . . . . . . . . . . . . . . . . 64

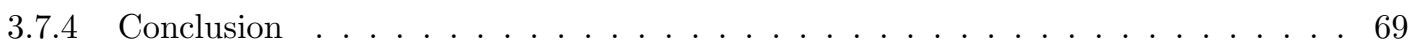

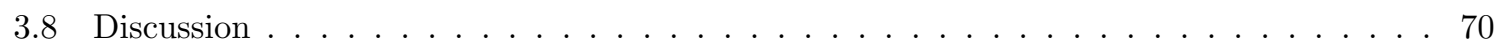

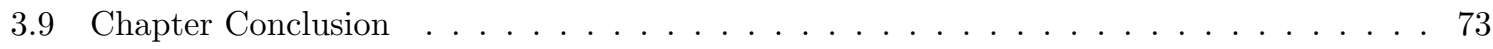

4 Algorithm Development $\quad 75$

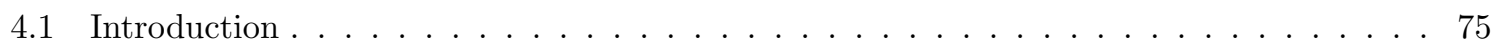

4.1.1 Conventional Melt Pool Dimensioning Techniques _ . . . . . . . . . . . . 75

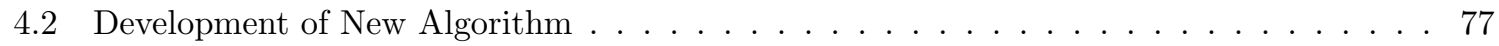

$4.2 .1 \quad$ Frame Averaging . . . . . . . . . . . . . . . . . . . . . . 78

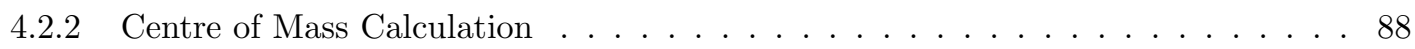

4.2 .3 Edge Detection . . . . . . . . . . . . . . . . . . . . 92

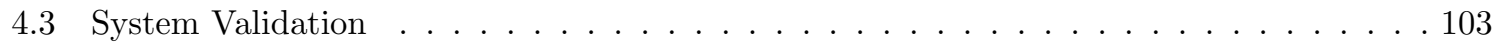

$4.3 .1 \quad$ Experimental Aim . . . . . . . . . . . . . . . . . . . . 103

4.3 .2 Experimental Methodology . . . . . . . . . . . . . . . . . . . . . 103

4.3 .3 Results . . . . . . . . . . . . . . . . . . . . . 105

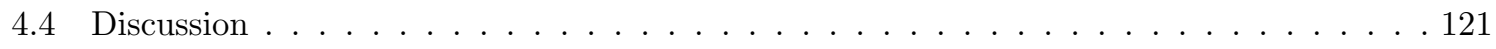

4.5 Chapter Conclusion . . . . . . . . . . . . . . . . . . . . 123

5 A Further Understanding of Laser Metal Deposition Systems 125

5.1 The Influence of Powder Mass Flow Rate on Melt Pool Width . . . . . . . . . . . . . . 125

$5.1 .1 \quad$ Experimental Set-up . . . . . . . . . . . . . . . . . . . 127

$5.1 .2 \quad$ Results . . . . . . . . . . . . . . . . . . . . . . 128

5.1 .3 Discussion . . . . . . . . . . . . . . . . . . . . 130

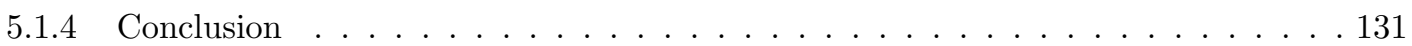


5.2 The Influence of Path Velocity on Melt Pool Width . . . . . . . . . . . . . . . . . 132

5.2 .1 Experimental Set-up . . . . . . . . . . . . . . . . . . . 132

5.2 .2 Results . . . . . . . . . . . . . . . . . . . 133

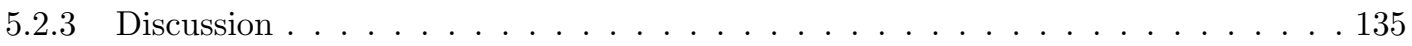

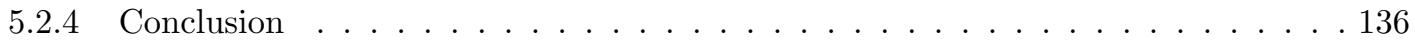

5.3 Chapter Conclusion . . . . . . . . . . . . . . . . . . . . 137

6 Melt Pool Monitoring of Different Materials 139

6.1 Calibration For New Materials . . . . . . . . . . . . . . . . . . . . . . 140

6.1.1 Methodology for Calibrating Different Materials . . . . . . . . . . . . . . . 142

6.1 .2 Experimental Methodology . . . . . . . . . . . . . . . . . . . 143

6.1 .3 Results . . . . . . . . . . . . . . . . . . . . 143

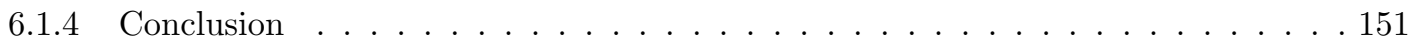

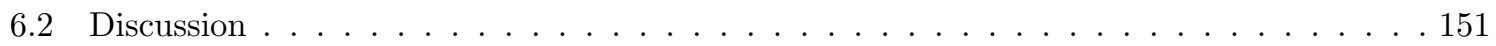

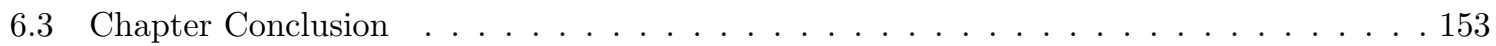

7 Conclusion $\quad 155$

8 Future Work $r$

8.1 Detailed Parameter Monitoring System . . . . . . . . . . . . . . . . . . 160

8.2 Future Work of Melt Pool Monitoring Systems _ . . . . . . . . . . . . . . 162

A Appendix - System Architecture Design and Algorithm Optimisation $\quad 181$

A.1 Practical Implications of Concept Systems . . . . . . . . . . . . . . . . . . 181

A.2 System Requirements . . . . . . . . . . . . . . . . . . . 181

A.2.1 Parameter Monitoring . . . . . . . . . . . . . . . . . . 182

A.2.2 Control Feedback . . . . . . . . . . . . . . . . . . . . 182

A.2.3 Defect Detection . . . . . . . . . . . . . . . . . 183

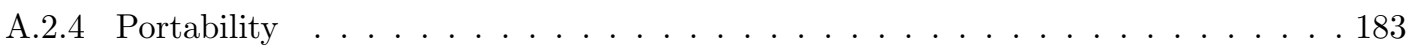

A.2.5 Adaptability . . . . . . . . . . . . . . . . . . . . . 184 
A.2.6 Processing Speed . . . . . . . . . . . . . . . . . . . . . . . . . 184

A.2.7 Data Storage . . . . . . . . . . . . . . . . . . . . . . . 184

A.3 System Architecture Design . . . . . . . . . . . . . . . . . . . . 185

A.3.1 The Layered Architecture Model . . . . . . . . . . . . . . . . . . . . . . . 186

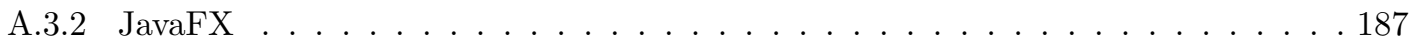

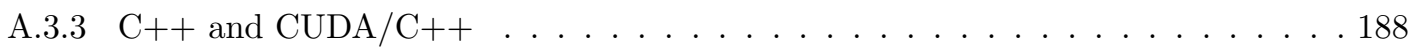

A.3.4 Final Architecture Concept . . . . . . . . . . . . . . . . . . . 188

A.3.5 Initial System Modules _ . . . . . . . . . . . . . . . . . . . . 193

A.4 Algorithm Optimisation . . . . . . . . . . . . . . . . . . . . . . 194

A.4.1 C++ Development . . . . . . . . . . . . . . . . . . . 194

A.4.2 CUDA/C++ Development . . . . . . . . . . . . . . . 197

A.4.3 Experiment - Speed Comparison . . . . . . . . . . . . . . . 198

A.4.4 Timing Results . . . . . . . . . . . . . . . . . . . 200

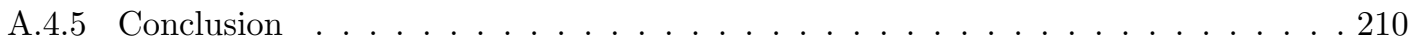

A.5 Graphic User Interface Design . . . . . . . . . . . . . . . . . . . . 211

A.6 Appendix Conclusion . . . . . . . . . . . . . . . . . . . . . . . . . . 212 


\section{List of Figures}

1 A schematic diagram representing a powder blown DED process. . . . . . . . . . . 12

2 Schematics representing: (a) Singular lateral powder injection delivery system. (b) Radially symmetrical powder injection delivery system. (c) Coaxial based powder delivery system. (d) Wire based delivery system . . . . . . . . . . . . . . . . 14

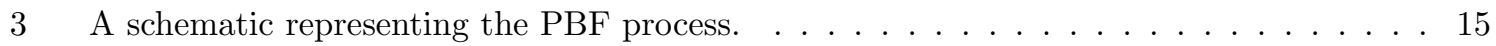

4 A graph displaying the increasing AM market revenue. . . . . . . . . . . . . . 18

5 A schematic representation of the trinocular set-up used to perform melt pool measurements in experiments carried out by Asselin et al. . . . . . . . . . . . . . . . . . . 21

6 A schematic representation of the coaxial CMOS-photodiode parameter monitoring systems used by Kruth et al. . . . . . . . . . . . . . . . . . . . . . . 22

7 A schematic representation of the off axis powder bed monitoring system used by

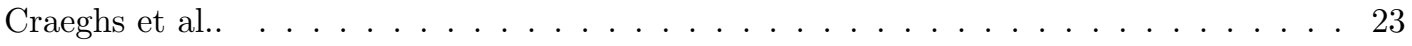

8 Image processing calculations of melt pool width using both the old (box) and new (ellipse) algorithms on three separate occasions. . . . . . . . . . . . . . . . . 25

9 A schematic representing the X-ray imaging systems used to study the dynamics of powder spattering in PBF processes. (a - entire system. b - close up) . . . . . . . . . 27

10 Diagrams representing: The deposition mode of a single layer (Left), the scanning mode after the production of a single layer (Middle), and the coordinate translations during scanning with axis reference (Right) . . . . . . . . . . . . . . 28

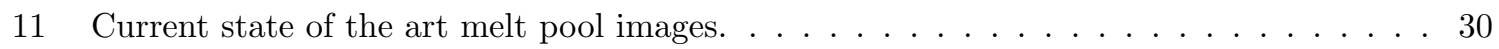

12 An image displaying the effects of long exposure times on moving objects . . . . . . 39

13 Left: A schematic representation of directional emittance from a flat surface. Right: Directional emittance of a blackbody and real body. . . . . . . . . . . . . . . . 41

14 Left: A high quality melt pool image achieved using a high-speed CCD camera. Right: A high quality melt pool image achieved using a high speed CMOS video camera. . . . . 41 
15 Left: CCD camera with C-mount mounting fixture. Right: Laser cladding head with exposed optical path. . . . . . . . . . . . . . . . . . . . . . . 44

16 The quantum efficiency of the CMOS sensor used for melt pool imagry. . . . . . . . . . 45

17 A graph showing the permitted wavelengths allowed through the UV/VIS cut-off filter. . 46

18 a) An image of the new camera installed on the laser deposition head. b) A schematic representing the stand off and FOV of the camera: (i) Laser deposition head. (ii) CMOS Camera. (iii) Melt Pool. (iv) Substrate . . . . . . . . . . . . . . . . . . . . . 47

19 A schematic diagram representing how directional emittance effects digital imaging of melt pools. .............................. . 51

20 a - exposure time $0.5 \mathrm{~ms}$. b - exposure time $1.0 \mathrm{~ms}$. c - exposure time $1.5 \mathrm{~ms}$. d - exposure

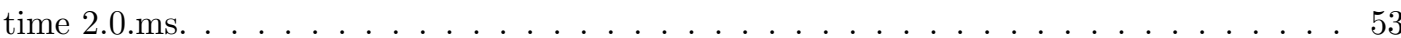

21 a: A typical melt pool image showing no directional emittance feature (exposure time - 2.0ms, LP - 600W). b: A typical melt pool image showing powder particles emitting large amounts of radiation (exposure time - 2.0ms, LP - 1200W). . . . . . . . . . 56

22 A melt pool still image displaying the melt pool dark region (exposure time - $1.5 \mathrm{~ms}$,

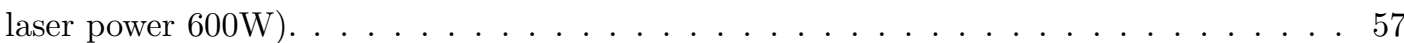

23 Melt pool images displaying the directional emittance feature. The exposure times for all images are 1.0ms. The laser powers are: a - 600W, b - 800W, c - 1000W, d - 1200W. 58

24 Four still images taken from a recording of a single line deposition displaying the issues of flares (exposure time - 1.0ms, laser power - 1200W) . . . . . . . . . . . . . 59

25 a - A still image from a recording of track deposition (exposure time - 0.5ms, laser power - 600W). b - A still image from a recording of track deposition (exposure time - 1.0ms, laser power $-600 \mathrm{~W}) . \ldots \ldots \ldots \ldots$. . . . . . . . . . . . . . 6 60

26 A still image taken from a video recording of track deposition showing a reduced number and magnitude of flares (exposure time - 0.5ms, laser power - 1200W). . . . . . . . . 61

27 A graph displaying the relationship between the low, best, and high exposure times and laser power settings in DED processes. . . . . . . . . . . . . . . . . . 65 
28 A series of melt pool images for various laser powers taken using the best exposure time. The laser power settings are: a.600W b.750W c.900W d.1050W e.1200W . . . . . . . . 67

29 A series of melt pool images for various laser powers taken using the low (*1) and high $\left(*_{2}\right)$ threshold exposure times. The laser power settings are: a.600W b.750W c.900W

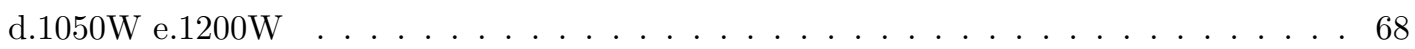

30 A series of images displaying the change in size and location of the high intensity pixel region at the centre of the melt pool for adjacent frames (exposure time - 0.55ms, laser power $-600 \mathrm{~W}) . \ldots \ldots \ldots \ldots \ldots \ldots \ldots$

31 A single frame taken from a melt pool video recording deposition at $600 \mathrm{~W}$ with an exposure time of $0.55 \mathrm{~ms}$. This image highlights bright regions around the edge and in the centre of the melt pool. . . . . . . . . . . . . . . . . . . . . .

32 A single frame taken from a melt pool video recording deposition at $600 \mathrm{~W}$ with an exposure time of $0.55 \mathrm{~ms}$. This image highlights long strips of saturated pixels caused by high speed powder particles. . . . . . . . . . . . . . . . . . . . .

33 A graphical representation of how the initial frames contribute to the averaging of frames. This diagram displays how raw images contribute to average images using a averaging window width of three. . . . . . . . . . . . . . . . . . . 83

34 A series of melt pool images that have been subject to the frame averaging technique. . 84

35 Images displaying the effects of varying $n$ for the averaging of melt pool images. a) $\mathrm{n}=$ 2 , b) $\mathrm{n}=3, \mathrm{c}) \mathrm{n}=7, \mathrm{~d}) \mathrm{n}=10$, e) $\mathrm{n}=15, \mathrm{f}) \mathrm{n}=25 \ldots \ldots$

36 Centre of mass locations over fifty sample frames using both the raw image feed and averaged image feed. . . . . . . . . . . . . . . . . . . . . 87

37 A graphical representation of the reduction of columns and rows for the averaged melt pool image. . . . . . . . . . . . . . . . . . . . . . . . . . . 89

38 A graphical representation of the scan arrays that were achieved using the reductions arrays subject to Equation 6 . . . . . . . . . . . . . . . . . . . . . 90

39 An image of the melt pool with the calculated COM. Radial distributions have been added to more easily distinguish equal distances from the COM location. . . . . . . . . 91

xiv 
40 An averaged image of the melt pool showing the north and south locations. . . . . . . . 93

41 Left: an image of the melt pool after frame averaging has occurred. Right: The raw signal that is extracted from the melt pool along the row at the COM-Y location. . . . . 94

42 Three graphs displaying the transition between the raw signal, averaged signal, and smoothed signal . . . . . . . . . . . . . . . . . . . . . . . 97

43 A figure displaying the smoothed signal with the calculated central intensity, lower threshold and upper threshold superimposed. . . . . . . . . . . . . . . . . . 100

44 A figure displaying the differentiated signal sections with calculated north and south

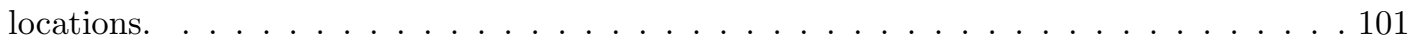

45 Left: The calculated north and south locations superimposed on the original melt pool image. Right: The corresponding smoothed signal used to extrapolate north and south

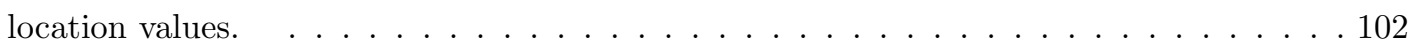

46 Top: An original signal of the melt pool width calculations for a recording of a track deposition. Bottom: The extracted signal used for melt pool dimensioning comparison. . 106

47 Top: An original signal of the melt pool width calculations for a recording of a track deposition. Bottom: The extracted signal used for melt pool dimensioning comparison. . 107

48 A graph representing the average melt pool width values for a range of different laser power settings. . . . . . . . . . . . . . . . . . . . . . . 108

49 Top: An original signal of the melt pool width calculations for a recording of a track deposition. Bottom: The extracted signal used for melt pool dimensioning comparison. . 109

50 Microscope images with corresponding measurements for different laser power settings.

a) $600 \mathrm{~W} \mathrm{b)} 800 \mathrm{~W}$ c) $1000 \mathrm{~W}$ d) $1200 \mathrm{~W} \ldots \ldots \ldots \ldots$

51 Microscope images with corresponding measurements for different laser power settings.

a) $600 \mathrm{~W}$ b) $800 \mathrm{~W}$ c) $1000 \mathrm{~W}$ d) $1200 \mathrm{~W}$. . . . . . . . . . . . . . . . . . . . . . . . . . . . . . . . . . .

52 a: An original melt pool image with calculated melt pool edges using the Canny edge detection technique. b: The melt pool binary image after it has undergone the binarisation, erosion and dilation processes. . . . . . . . . . . . . . . . . . 115 
53 An image showing two incorrect melt pool edge locations using the Canny edge technique. a: Original Image. b: Binary Image. . . . . . . . . . . . . . . . . . . . . . 117

54 An image showing the north and south locations calculated using the directional emittance technique for frames in Figures 52 (a) and 53 (b) . . . . . . . . . . 117

55 Still frames taken from video recording tracks deposited at 600, 900 and 1200W. Red lines indicate the calculated north location, green lines indicate the calculated south

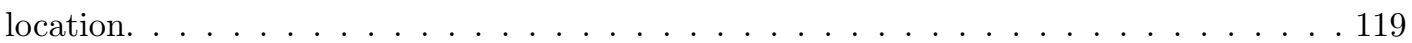

56 A still frame taken from a video recording with its corresponding smooth signal. . . . . 120

57 A detailed image of the melt pool displaying the smooth gradient at the edge of the melt pool that the directional emittance image processing technique could not resolve. . 121

58 A graph representing all calculated average melt pool values for various parameter settings used in the PMFR experiment. . . . . . . . . . . . . . . . . . 128

59 A graph representing all calculated average melt pool values for various parameter settings used in the path velocity experiment. . . . . . . . . . . . . . 134

60 A series of images comparing melt pool saturation levels for a range of different laser power settings. $a \& b=600 \mathrm{~W}, \mathrm{c} \& d=750 \mathrm{~W}, \mathrm{e} \& \mathrm{f}=900 \mathrm{~W}$. All melt pools on the left are EN25 melt pools and all melt pools on the right are 316L melt pools. . . . . . . . . . . . 141

61 A graph representing part of the image processing algorithm. The smooth signal is extracted from the incorrectly measured frame, and the upper and lower thresholds used to extract the directional emittance signal are displayed. a) Thresholds attempting to measure the south location. b) Thresholds attempting to measure the north location. . 145

62 A melt pool image frame that gave an incorrect south location. . . . . . . . . . . . 146

63 Snippets extracted from the melt pool width calculations for tracks depositing at various laser power settings. . . . . . . . . . . . . . . . . . . . . . 148

64 a: A still image taken using the exposure time selected by the operator for the material 316L. b: A still image taken using the exposure time selected by the operator for the material EN25. The laser power setting for both images is $900 \mathrm{~W} \ldots$. . . . . . . . . . . 148

65 Average melt pool readings over different laser power settings for the material 316L. . . 149 
66 A graph to compare average melt pool widths for 316L and EN25. . . . . . . . . . 150

A.1 A schematic diagram representing the architecture structure design to be used for further system development. . . . . . . . . . . . . . . . . . . . . . . . 190

A.2 A schematic diagram representing a future architecture expansion. . . . . . . . . . . 192

A.3 Information about the GPU used to develop the new image processing algorithm. . . . . 200

A.4 A screenshot of the final GUI design implemented into TWI's industrial environment. . 212 


\section{List of Tables}

1 The conversion rates between machine percentage and grams per minute for EN25. . . 49

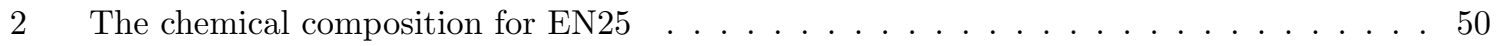

3 Laser power and exposure time settings for preliminary exposure experiment $\ldots \ldots \ldots 5$

4 Average measured track widths . . . . . . . . . . . . . . . . . 111

5 The matrix of parameters used in the PMFR experiment. . . . . . . . . . . 127

6 The matrix of parameters used in the path velocity experiment. . . . . . . . . . 133

7 Chemical composition of SS 316L powder used in Chapter 6 deposition . . . . . . . . . 140

A.1 The average time taken to execute the image process algorithm using CPU and GPU techniques with their corresponding frame rates. . . . . . . . . . . . . . . . 201

A.2 Detailed timing results showing the executing times for both the circular ROI and frame

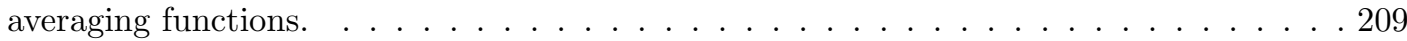




\section{Introduction}

\subsection{Motivation for Research}

Additive Manufacturing (AM) is the industry standard term for all applications of the technology (ASTM F2792). It is defined as the process of joining materials to make objects from 3D model data, usually layer upon layer, as opposed to subtractive manufacturing methodologies [1]. Synonyms include additive fabrication, additive processes, additive techniques, additive layer manufacturing, layer manufacturing, and freeform fabrication [1].

$\mathrm{AM}$ is a collective group of dynamic manufacturing processes where techniques, materials and components vary depending on the desired application. AM techniques have taken many different forms and produce components that range from small plastic desk ornaments, to large aerospace parts currently being used in the Trent XWB-97 engine [2]. Techniques vary from the injection of material into the pathway of a laser beam - otherwise known as Direct Energy Deposition (DED), to Stereolithography (SL) - the use of photopolymerisation to link chains of molecules together.

AM has many advantages and disadvantages that vary depending on the technique used to fabricate the desired component. Whilst differences between techniques are present, certain advantages and disadvantages can be generalised for the collective group of manufacturing techniques.

One advantage of AM is that it promotes flexible design, as many constraints that are present in subtractive manufacturing techniques no longer exist [1]. AM is optimised for low volume production, and geometrical variations can be made between different components without the need to manufacture new moulds or tools. The ability to manufacture immediately after the design process greatly reduces the lead time in production. AM can produce moving parts and assemblies within single builds. The amount of waste in an AM process is also greatly reduced compared to subtractive manufacturing and in some cases all material deposited is used in the end component.

With every manufacturing process there are also disadvantages. AM can suffer from slow build rates. Whilst the technique can drastically reduce lead time, the increased design flexibility of AM often comes with the cost of increased manufacturing times, particularly for large batch production. 
Comparing the material extrusion AM technique to conventional injection moulding, one would expect to see production to be magnitudes faster on the later. AM machines can also require large capital investment and the cost of materials used in AM are often higher than the same materials used in conventional manufacturing techniques. This is due to AM materials often having to undergo extra processing to form specific material shapes such as fine powder particles and extrusion filaments [1]. AM fabrication techniques can also limit the size of components, and post processing is often required. AM also suffers from the disadvantage of requiring skilled workers with in-depth knowledge of specific AM processes to operate machinery. Parameters need to be optimised, which can be incredibly difficult to do. Without the optimisation of parameters, and even sometimes with, AM fabrication processes can produce parts with poor mechanical properties. The repeatability of AM components is also known to be poor.

Much research has been directed towards the improvement of AM processes, but rarely has research aimed to improve all AM techniques due to the sheer diversity between them. Research usually focuses on the development of one family of AM techniques. The focus of this research studies in detail DED processes. DED processes are metal-based AM process that uses an incident laser on a substrate, combined with an introduced material (usually powder or wire) to produce a molten metal pool that fuses with the substrate. This molten pool (melt pool) of injected metal mixed with underlying substrate is built up using robotic machinery to produce 3D components in a layer by layer fashion. This technique experiences all the advantages and disadvantages that are explained above for AM techniques in general. One of the most prominent disadvantages that the DED process experiences is the requirement for a skilled operator and the demand for optimum parameter settings. Nonoptimised parameter settings can often result in build defects, build failures and components without the required mechanical properties for the application. This research focuses on this disadvantage and aims to enhance the DED process by producing techniques that can be used to study the complex problems of optimising parameters. The techniques also aim to be used as tools to perform such optimisations in industrial environments. DED techniques are explained in detail in section 2.2.1.

Optimising parameter settings in DED processes is difficult and usually requires a long trial and error iteration period. Technical staff that operate these machines in industrial environments regularly 
deal with new materials and geometries. These require a constant change in parameter settings and operators often undertake multiple builds before optimised parts can be used for production. Even after the parameter iteration period where optimal parameters are calculated, certain variations in the DED process result in the manufacturing process having poor repeatability. Substrate heat accumulation, variations in laser power, acceleration/deceleration periods in robot position translations and uneven Powder Mass Flow Rate (PMFR) are just a few examples of variations that can cause poor repeatability in the DED process. Over fifty parameters are known to effect AM builds, and changes in one parameter can have a cascading effect on multiple parameters in the build [3]. Complex parameter interactions, when studied in detail and understood to enable manufacture of a specific component, are completely changed when a new material or geometry is introduced. Monitoring parameters in DED has proven to aid in the selection of optimised parameter settings, reduce the time taken to optimise parameters, and gain a better understanding of the complex DED process. Parameter monitoring systems have also been used in the development of AM control systems that can change parameters in-situ to counteract issues such as heat accumulation and variations in build height. This subsequently produces higher quality components. Parameter monitoring systems vary and include PMFR monitoring, powder particle speed monitoring, melt pool monitoring and deposition height monitoring. A detailed analysis of these techniques will be discussed in the literature review (Chapter 2). This research will use melt pool monitoring techniques to study DED parameters in detail to give a better understanding of the complex process. This research will create a system that can be used to monitor melt pools in the DED process in an industrial environment. The newly developed melt pool monitoring system has improved melt pool image quality and can reliably measure melt pool width, without the need for emissivity values. This technique can be used on multiple materials after minor calibration procedures.

Melt pool monitoring is a technique used in AM in both DED and Selective Laser Melting (SLM) processes. The technique uses equipment to monitor properties of the melt pool that are governed by input parameters. Properties of the melt pool have received much scientific research, and the melt pool width forms the focus of this thesis. The specific type of melt pool monitoring researched and developed here is melt pool monitoring using machine vision cameras. The melt pool can be monitored in the DED process using cameras with correct settings, properties and filters to produce images of the 
molten metal during DED build cycles. Images captured from the installed machine vision cameras are often subjected to image processing algorithms to extract the relevant melt pool features. Changes in input parameters often lead to changes in the melt pool and melt pool monitoring can be used to track these changes for any discrepancies. Melt pool monitoring has been used to produce control systems that change parameters in-situ and to understand in detail how parameter alterations effect the final component.

Whilst melt pool monitoring systems can offer advantages when installed on DED systems, they are not widely used in industrial environments due to certain disadvantages. Academic research has utilised these systems, but inherent flaws in current designs have stopped the adoption of these techniques into industrial applications. The current systems have limited accuracy due to the image processing techniques used to extract melt pool dimensions. The current technique is deemed inaccurate due to the use of emissivity values and poor machine vision techniques. The introduction of a new material into the DED process requires new emissivity values due to them being material dependant. These emissivity values require complex experiments to calculate, and the accuracy of these values are known to be poor. Due to inaccurate systems that require long calibration procedures, the adoption of these techniques into industrial environments has been limited.

Currently there is no system that can accurately measure the melt pool width without the need for emissivity values and that can transition between materials with little to no calibration in DED systems.

\subsection{Principal Hypothesis}

Optimising melt pool imagery will highlight features that will indicate the true melt pool's edge. A new melt pool image processing technique will be able to use edge features to calculate melt pool width more accurately than the current techniques, without the need for emissivity values. The new image processing technique will be able to resolve changes in melt pool geometry, with varying parameters, in more detail than the current image processing techniques. The new algorithm will provide new information about the effects of parameter changes on melt pool geometry. The new algorithm will be able to measure melt pool widths for different materials without the need for lengthy calibration 
procedures.

\subsection{Aims and Objectives}

The aim of this research is to produce a novel technique that can more accurately measure melt pool widths in DED systems without the need for material dependant emissivity values. With consideration of the current state of the art melt pool monitoring techniques and overall project aim, the discrete objectives of this research are as follows:

1. To optimise machine vision imaging techniques and improve melt pool image quality.

2. To produce a new and improved melt pool width dimensioning technique with increased accuracy.

3. To eradicate the need for inaccurate emissivity values in melt pool algorithms.

4. To better understand the complex interaction of process parameters.

5. To invent an algorithm that can measure multiple materials with minimal calibration procedures.

6. To produce software to encompass newly developed techniques, capable of future expansion.

7. To optimise algorithm execution speeds for future hardware upgrades and control system development using Graphics Processing Units (GPUs).

\subsection{Research Methodology}

To analyse the improved machine vision imaging techniques, new melt pool images are compared with current state of the art melt pool images to establish the presence of edge features. The presence of edge features in melt pool images validates that the new optical techniques can better capture details of the melt pool.

To compare the new algorithm with the current state of the art, average melt pool width calculations for whole tracks are directly compared with each other for different laser power settings. Individual frames are extracted with melt pool width measurements superimposed upon them to establish algorithm accuracy. Measured average melt pool widths, calculated using both algorithms, are 
also compared with hand calculations and solidified track width measurements. Differences between these measurements are used to validate and highlight limitations in both image processing techniques.

The new technique is used in subsequent parametric studies to better quantify how both PMFR and path velocity effect melt pool width. This is done by recording single line track deposition, with various parameter settings, and comparing calculated melt pool width averages. All parameter settings use cases are plotted onto single graphs and analysed.

The new technique is used to determine the interaction between laser power and melt pool width for a different material. A new material is introduced and the new algorithm is used to calculate melt pool width. The steps required to calibrate the new technique for different materials is detailed and changes to optical acquisition settings and underlying algorithm are made. The calibrated technique is subsequently used to perform a parametric study with varying laser power settings, allowing for the direct comparison of two materials.

The optimisation of algorithm execution speed is analysed by timing both the original and GPU implemented algorithms. Both algorithms are designed in the same language to allow for direct comparison. Algorithm optimisation and software development is detailed in Appendix A.

\subsection{Summary of Contribution to Knowledge}

This research has optimised machine vision imaging techniques in melt pool monitoring systems and highlighted the need for adaptive exposure times. A new technique has been developed that allows for high quality melt pool images, with detailed edge features, at a range of laser power settings. A new algorithm has been developed that uses the newly discovered edge features to calculate melt pool width more accurately than current image processing techniques, without the need for emissivity values. Parametric studies have allowed for a detailed understanding of melt pool behaviour in DED systems and uncovered parameter interactions that have yet to be documented. The new system is able to measure different materials with minimal calibration procedures, allowing for the direct comparison of powder materials. This technique has been optimised using GPUs, and software has been developed to house these academic advancements. 


\subsection{Organisation of Thesis}

This research begins by analysing current literature and highlighting the limitations of state of the art melt pool monitoring techniques. The scope of this work then focuses research into two main technical developments.

The first area of development focuses on the optimisation of machine vision imaging techniques. This technical development improves image acquisition and enhances melt pool edge feature details.

The second technical development creates a new image processing algorithm to extract melt pool width. This newly developed algorithm aims to improve dimensioning accuracy and eradicate the need for emissivity values.

These technical advancements are then utilised to provide a better understanding of the complex interaction of AM parameters by performing a series of parameter experiments. These are used to determine the influence that different parameter settings have on melt pool geometry.

The new technique is then used to measure melt pools of different materials, whilst providing a suitable minimal calibration procedure. These technical advancements are then used to compare the influence of parameters across multiple materials. 


\section{Review of Literature}

\subsection{A Brief History of Additive Manufacturing}

The first concept of AM dates back to 1987 with the initial commercialisation of SL [4]. The original AM system was developed by 3D systems and was named the SLA-1, before being further developed into the more popular models such as the 'SLA 250' and the 'Viper SLA' [1]. SL was the first technique used in the AM industrial sector and the print materials were limited to certain type of polymers.

Additional techniques were developed in 1991 with the creation of three new AM methods. Fused deposition modelling was created by Stratasys, Solid ground curing was created by Cubital and Laminated object manufacturing was created by Helisys - all within the same year. Fusion deposition modelling was the first introduction of an extrusion AM technique, where thermoplastic filaments were melted and deposited to produce three-dimensional parts. The solid ground curing technique passed UV light through masks, created with electrostatic toner on a glass plate, to cure whole layers of UV-sensitive liquid polymers - very similar to today's SL techniques. Laminated object manufacturing was a method of creating three-dimensional products by layering up sheet metal - a process now categorised as sheet lamination within the current British standards [1].

In 1992 the AM technique Selective Laser Sintering (SLS) was made commercially available by Teijin Seiki [1]. SLS being a process that uses a laser to melt specific regions of metal powder that are located on a powder bed. The laser fuses together powder particles to form a layer whose shape is dependent on geometric inputs. When a layer is complete, the powder bed then descends into the build chamber - before scraping another layer of powder across the entire powder bed. The processes of melting powder and introducing new powder layers is then repeated to produce 3D components. The SLS process today is categorised as a Powder-Bed Fusion (PBF) process and is widely used in the field of AM.

Another historical highlight is the introduction of direct metal deposition in 2002 by Precision Optical Manufacturing [1]. Direct metal deposition is an AM method that uses laser power to melt substrate material to form a molten metal pool - before introducing metal powder particles using a 
carrier gas. The metal powder particles interact with the melted substrate and laser to form a melt pool that subsequently cools to form solid material. This process can be moved using robotic systems to create three-dimensional structures.

In 1993 the first binder jetting AM system was created by Soligen and was called direct shell production casting. This system used an inkjet mechanism to deposit a liquid binder onto ceramic powder, forming shells that can be used in the investment casting process [1].

In 1994 the first material jetting AM system was created by Solidscape and was named the ModelMaker. This system used an inkjet head to deposit wax structures in a similar way to the material extrusion process [1].

The last major development to date in AM was the development of the Electron Beam Melting (EBM) process in 2006 by Arcam. The EBM process is very similar to the Selective Laser Melting (SLM) as they are both PBF techniques. The fundamental difference between the two techniques are the energy sources used in the process. EBM uses an electron beam instead of a laser beam as an energy source. British standards today categorise both EBM and SLM as PBF processes [1].

Subsequent developments in AM are progressive changes rather than step changes. Further developments in AM have improved process capabilities, but the alterations of the actual conceptual manufacturing ideas are very small in comparison to these mentioned. This chapter has highlighted the invention of the main types of AM. A detailed history of AM techniques with price comparisons can be found in Wohlers Report [1].

\section{$2.2 \quad$ Additive Manufacturing Processes}

AM includes a range of different processes and subsequently has introduced a long list of name variations. SL for example is also known as optical fabrication, photo-solidification, solid free form fabrication, solid imaging, rapid prototyping, resign printing and 3D modelling. DED can also be called 3D printing, rapid prototyping and free form fabrication. The extensive number of acronyms and differing names for similar (if not the same) processes can cause confusion as to the exact functionality of the discussed techniques. The considerable number of names given to similar processes is a behaviour that is seen throughout the industrial sector due to the infancy of AM standardisation. AM is a young 
industry that has been explored by many different companies that use similar but not identical terminology for their individual systems. Progress is being made towards the standardisation of terms, and the British Standards Institute (BSI) keeps up to date documents with the standard terminology [5]. Standardisation has been somewhat successful by categorising AM techniques into seven categories; Binder Jetting, Direct Energy Deposition, Material Extrusion, Material Jetting, Powder Bed Fusion, Sheet Lamination and Vat Photopolymerization. These seven are listed in the following BSI Standards Publication [5].

As stated in the introduction, the focus of this research is on DED processes. Literature concerning other AM processes allows for a broad view of developments made to AM systems. Many similarities can be drawn between different AM processes. Certain DED and PBF processes involve a melt pool, heat source, gas environments, metallic powder and similar parameters. For this reason, the literature review will encompass various AM techniques and focus more on the system developments rather than the techniques themselves. The following sub-sections will outline the DED and PBF processes in detail due to the abundance of relative literature surrounding them.

\subsubsection{Direct Energy Deposition}

DED is an AM process that produces three dimensional components by creating a molten pool on the surface of a material using an laser, before introducing material using either a carrier gas or wire feeder. The fundamental concept of the DED process is pictured in Figure 1.

Figure 1 shows a two dimensional schematic diagram on the left hand side and a three-dimensional representation of the build process on the right hand side. The left hand side of the image schematically displays the different components including; a laser beam (energy source), a powder stream (material to be added), shielding gas, deposited material, a melt pool, and a work piece (substrate).

The DED process focuses a laser beam through optics onto a substrate to create a molten metal pool. A stream of material shielded by argon gas then injects small powder particles (ranging from approximately $37 \mu \mathrm{m}$ to $150 \mu \mathrm{m}[7]$ ), or wire into the melt pool causing material to build up. This deposition technique causes fusion bonding between the added material and substrate. The laser cladding head has at least three degrees of freedom allowing the cladding head to move in $\mathrm{X}, \mathrm{Y}$ and $\mathrm{Z}$ 


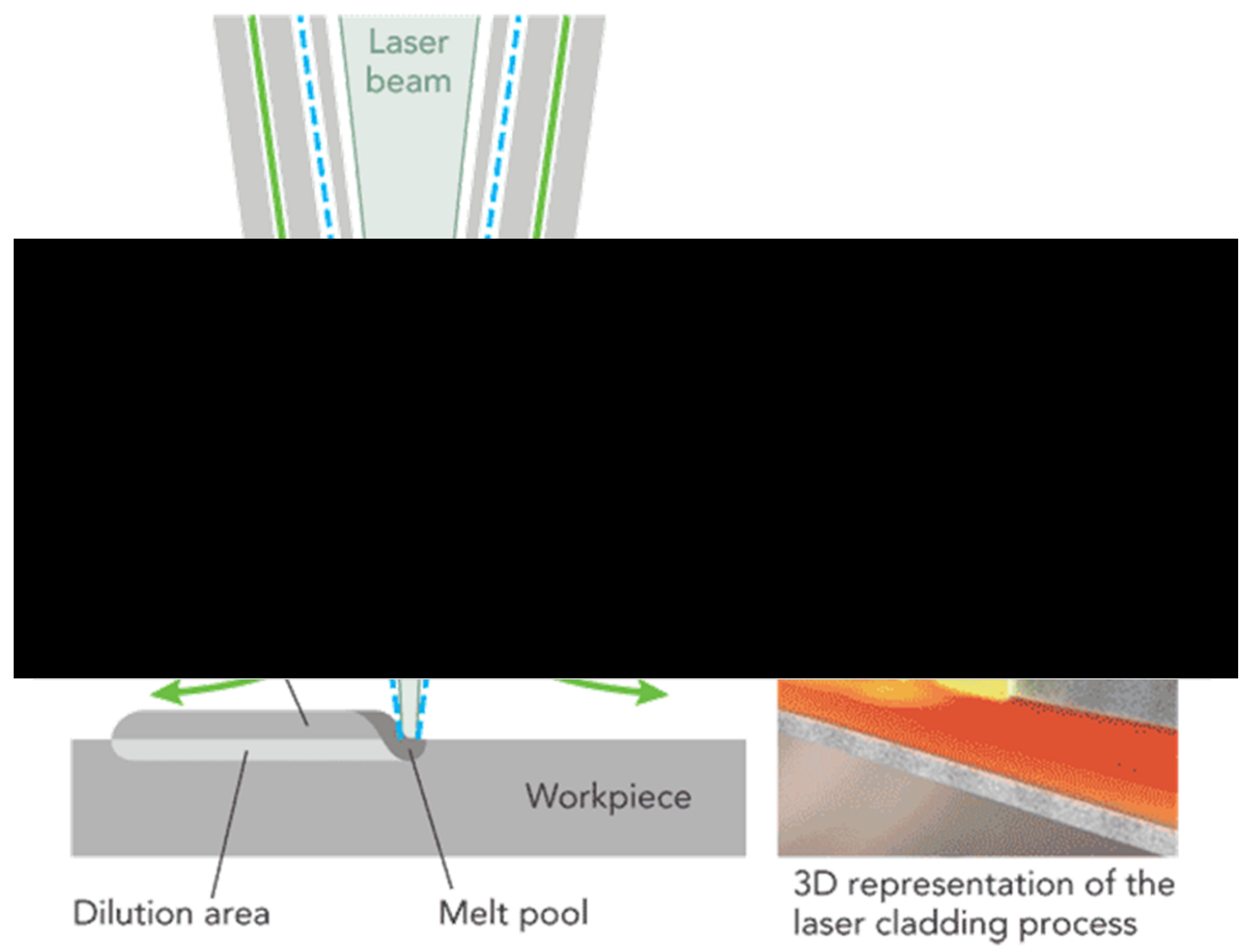

Figure 1: A schematic diagram representing a powder blown DED process [6].

directions. The cladding head moves in the $\mathrm{X}$ and $\mathrm{Y}$ directions to deposit a complete 2D layer before the system translates in the $\mathrm{Z}$ direction. This process is repeated to construct three-dimensional parts from two dimensional layers.

Like with nearly every other AM technique, the DED technique comes with variations depending on what manufacturer produces the system. One of the variations that different systems have is the degrees of freedom available. Early powder blown AM machines were limited to three degrees of freedom with the laser cladding head only being able to perform $\mathrm{X}, \mathrm{Y}$ and $\mathrm{Z}$ translations. DED techniques of today more commonly have more degrees of freedom. Typically 5-6 degrees of freedom for the cladding head, and an additional 2-3 for substrate manipulation. This allows for greater geometric 
freedom in the build process and radially symmetrical parts to be built more fluidly. The sponsoring company TWI have multiple in-house DED systems with six degrees of freedom.

Another variation that may be seen in DED machinery is the energy source used to create the melt pool. There are many different types of lasers used in DED systems. The laser systems in DED processes can vary and include $\mathrm{CO}_{2}$, Yb-fibre and Nd-YAG lasers [1].

The last major alteration between different DED systems is the powder/wire delivery system. Figure 2 shows four different material delivery systems. Figure 2a shows a lateral powder injection system that is situated next to, but not inside, the laser cladding head. This was one of the earlier powder feeding designs and often produced limited quality AM components. This design was expanded on (Figure $2 \mathrm{~b}$ ) with multiple radially symmetrical powder nozzles situated exterior to the cladding head. These nozzles feed material simultaneously into the melt pool. This delivery method was again improved with the creation of a coaxial powder delivery system shown in Figure 2c. This system allows for powder to be equally distributed into the melt pool and has been proven to raise both efficiency and quality of the clad. The last material delivery system in this diagram uses wire feeds. The external wire delivery system feeds wire straight into the melt pool to build three-dimensional parts. Wire based AM is still used today and has the benefit of reduced cost and reduced percentage of wasted material (as powder will often miss the melt pool). Wire fed AM has had success with larger manufactured components but tends to have less resolution when compared with powder-based AM processes. 


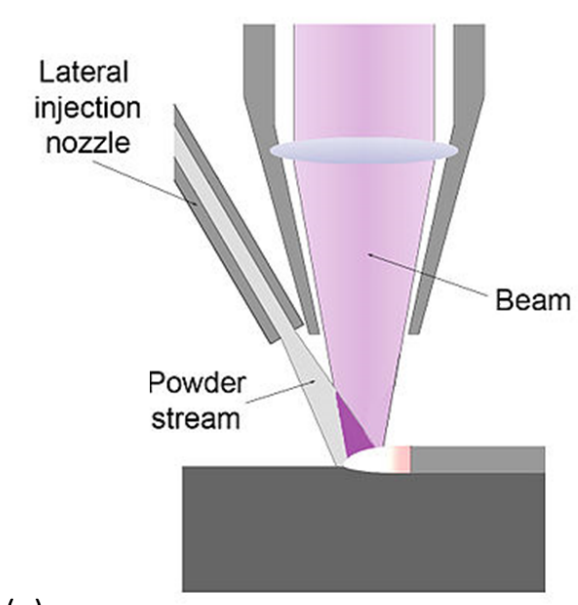

(a)

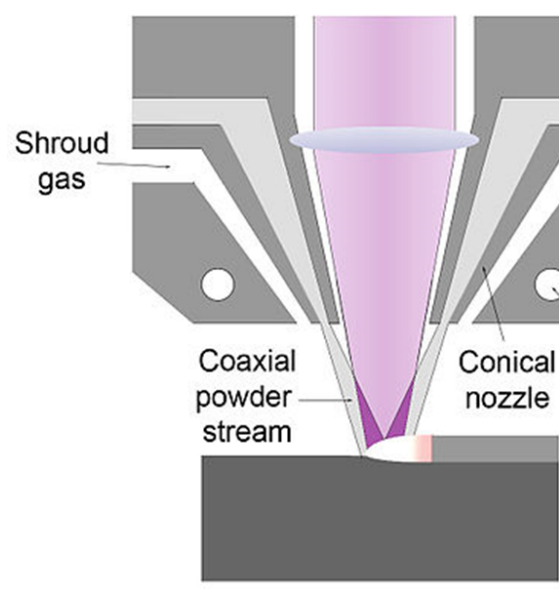

(c) (b)
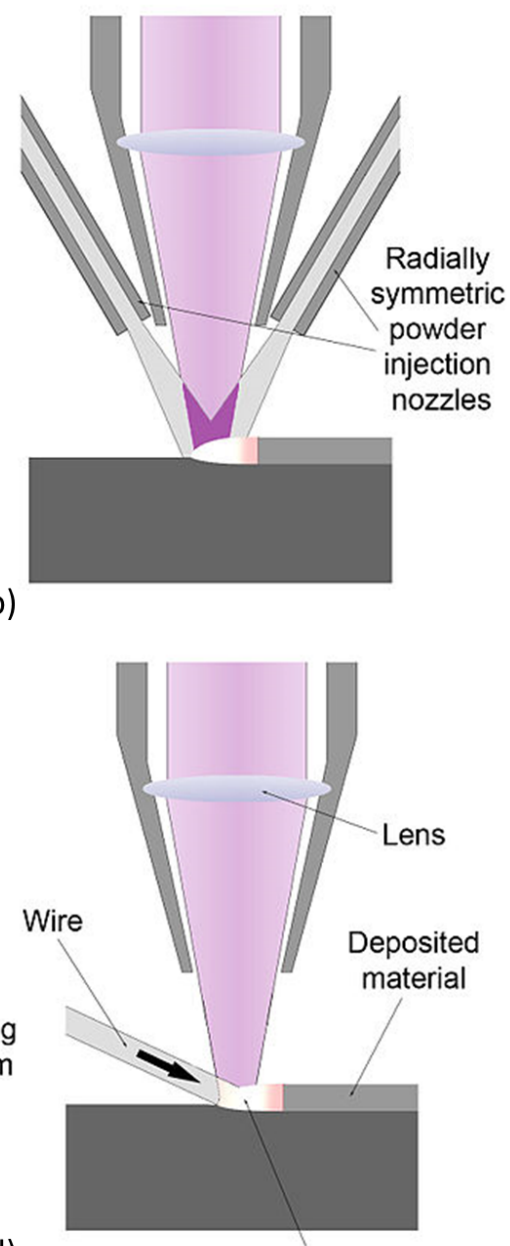

Meltpool

Figure 2: Schematics representing: (a) Singular lateral powder injection delivery system. (b) Radially symmetrical powder injection delivery system. (c) Coaxial based powder delivery system. (d) Wire based delivery system [8].

\subsubsection{Powder Bed Fusion}

PBF methods encompass all techniques that use focused energy, in either electron beam or laser beam form, to selectively sinter or melt layers of powder situated in a powder bed. The names of PBF techniques include EBM, SLS, laser beam melting, SLM, and direct metal laser sintering. Powder-bed 
fusion techniques can be used to produce both metallic and polymer components.

The technique uses either a high-powered laser, or an electron beam, as the energy sources to melt a layer of powder situated on a bed. PBF uses CAD/CAM software to slice a three-dimensional CAD design into layers. Layers are then produced by translating the machines power source in the $\mathrm{X}$ and $\mathrm{Y}$ planes to fuse powder particles together in accordance with the sliced CAD design. Once a single layer in the $\mathrm{PBF}$ process is completed, the build table descends in the $\mathrm{Z}$ direction by a single layer thickness and powder is evenly redistributed using a roller/blade. Once a new powder layer has been laid down, the next layer can be fused together. This cycle repeats itself until all layers are fused and the entire component is built. At the end of the build, the fabricated component will be surrounded by excess powder, which has been used as support material throughout the build. This powder is then removed and brushed off to be reused in future builds. The EBM technique varies from other PBF techniques by using electron beams instead of lasers for the heat source. EBM machines conduct builds in a vacuum chamber and the build times are reduced when compared with other PBF techniques [1].

Figure 3 displays a schematic representation of PBF process.

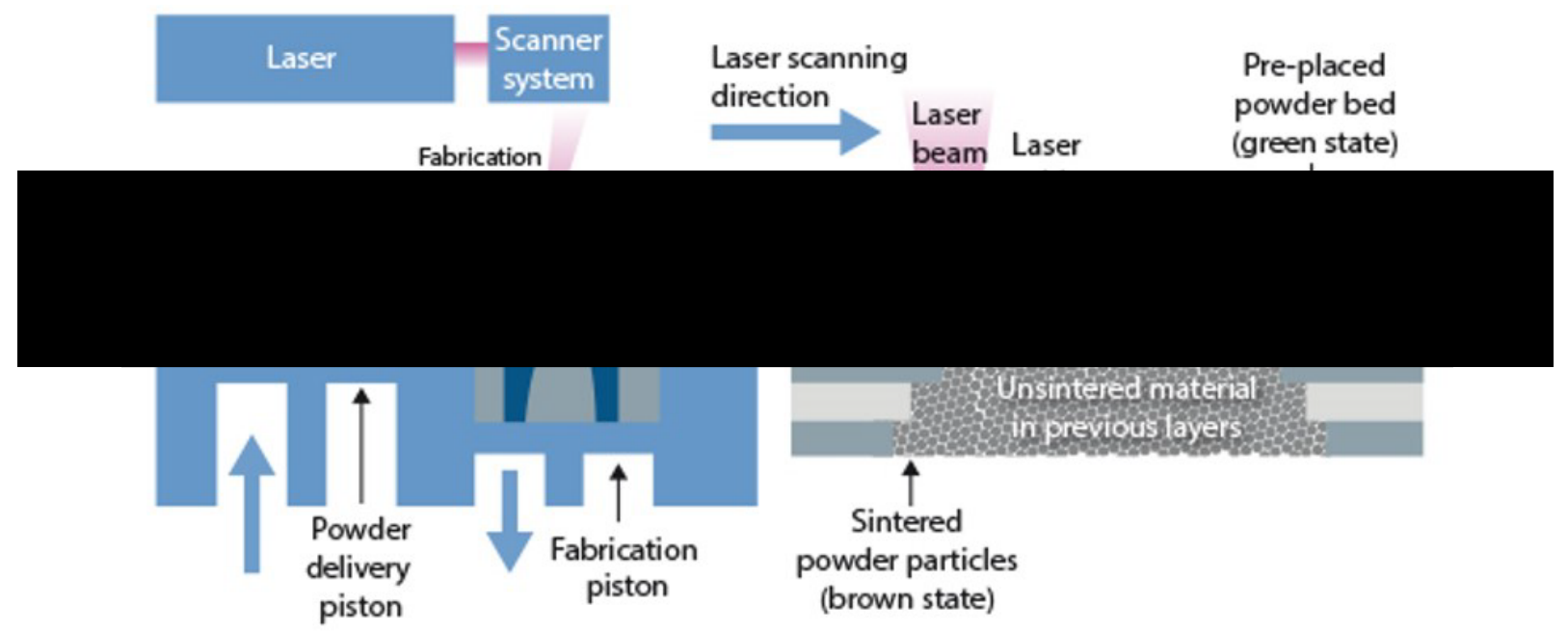

Figure 3: A schematic representing the PBF process [9]. 


\subsection{Metal Powders in Additive Manufacturing}

AM can produce products in a range of different materials, however, the two main material groups that are used in industrial processes are metals and polymers. Composite, ceramic and ceramicmetal hybrid components are achievable through AM processes, but require a more specialist process approach. This research will focus on the use of metal powders.

There are a wide range of metallic powder materials available for AM processes including stainless steels, tool steels, titanium alloys, commercially pure titanium, aluminium alloys, cobalt-chromium alloys, gold, silver and copper-based alloys [1]. The materials that can be used in AM expand each year and metallic AM system manufacturers support the development of new materials. Certain PBF and DED processes can be used to produce metal components, with the DED process having slightly more material flexibility. DED processes have also been used for the deposition of multiple materials within a single build, something that PBF cannot do [1].

Most metallic AM processes use powders as a material feed. All of which are exclusively made through the atomization process [1]. Atomization is the process of melting metal and disintegrating the material with an inert gas. Plasma torches located at the top of an atomization chamber provide the heat to liquefy the metal, and as the molten metal falls, solidification occurs turning the liquid into a spherical powder. Alterations to the gas injection systems, melting area, and nozzles can be undertaken to achieve increased uniformity, different particle diameters, or more spherical shapes. Water atomisation is the dominant technique for powder production, but causes irregular shapes in powder due to the quick solidification cycle [1].

Comparing DED and PBF techniques, the PBF technique is the most demanding with regards to powder regulations. The PBF process requires a powder bed with spherical powder particles that are uniform in size and can create a flat, dense layer of powder [10]. The DED systems tend to have less strict powder requirements [1].

Atomization can produce powder particles with size ranging from $<5$ microns to 240 microns. The less strict powder blown process can use powder particles larger than 100 microns, but certain PBF machines such as the Arcam's EBM system requires powder to be in between 45 and 100 microns [1]. 
The optimised powder size for the majority of PBF machines is $30-45$ microns. It should be noted that PBF systems work better with a narrow distribution of powder sizes to create a flat uniform bed [10]. By not regulating the powder particles, defects such as porosity can occur in AM components [1].

The cost of metals powders in AM will be a major factor in the growth of the AM industry. Metal powder production can be carried out by both the AM system distributors and a range of specific metal powder production companies. This allows the user to more independently control both the cost and quality of their manufactured components. Depending on the price of labour, powder costs are the second or third largest financial contributing factor to metallic AM [1].

The two largest determining factors in the production costs of powder particles are firstly the size yield and secondly the cost of argon. The size yield varies for different machines with EBM systems only being able to use around 50\% of the overall powder produced (powder range 45 - 100 microns), and Laser PBF systems being able to use even less at 20\% (powder range 30 - 45 microns) [1]. Nearly one third of metal powder produced cannot be used in PBF processes which is of high importance when PBF sales outweigh DED sales by seven to one. Over $60 \%$ of the production cost of AM powder can be attributed to argon gas but using argon gas has the added advantage that it can be recycled with additional resources [1].

\subsection{The Economics of Additive Manufacturing}

$\mathrm{AM}$ has begun to dominate the news within engineering due to its future potentials in a range of different markets including aerospace, medical/dental, automotive and robotics. The advantages of AM have been noted by industrial companies, and many of them are looking to increase their AM capabilities. GE predicted a 14\% annual growth for AM through 2017 [11] and the AM industry surpassed $\$ 5.1$ Billion in 2016 [1]. The number of AM related articles published rose from 1,600 in 2011 to 16,000 in 2012, and Figure 4 shows the increase in AM markets worth from 2011 to 2016. Government funding of AM research has been seen globally - with China, Singapore, and Germany heavily investing in the technology [12].

The Chinese government pledged $\$ 245$ million to a seven-year project designed to advance AM technology and Singapore started investing $\$ 500$ million over five years into AM in 2014 [1]. In the 


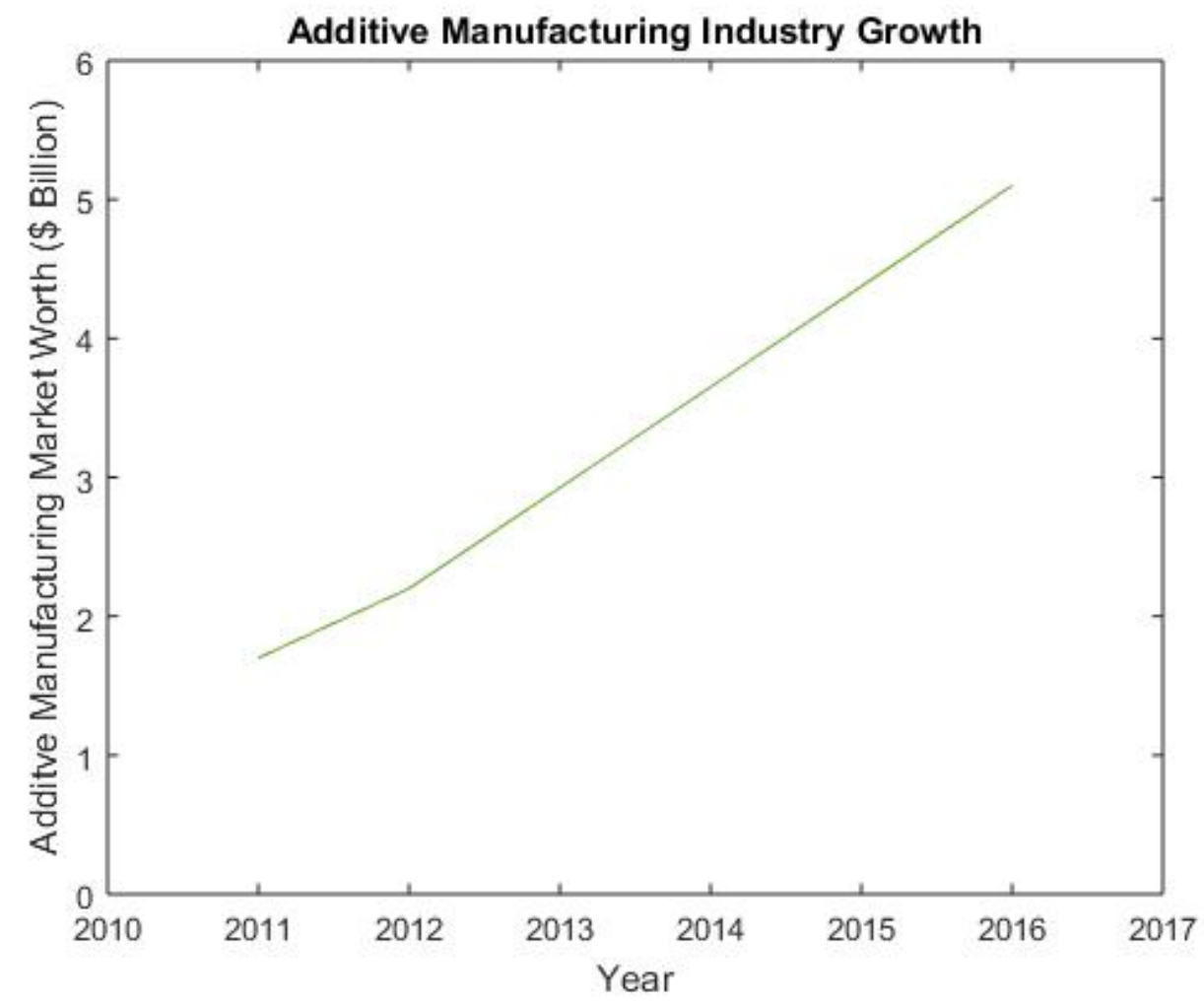

Figure 4: A graph displaying the increasing AM market revenue $[1,12]$.

USA various government funded institutes such as the National Institute of Standards and Technology (NIST) have programs to advance AM technologies in multiple ways including: research process methodology; process optimisation and control; advanced sensor systems; materials characterisation; data formats; and standards development. NIST awarded $\$ 7.4$ million worth of grants in 2013 [12]. Advancements in materials, new design tools and the expiration of key patents has aided in the constant growth of AM.

\subsection{Parameter Monitoring in Additive Manufacturing}

The focus of this research is the development of parameter monitoring systems in AM (specifically DED) processes. Parameter monitoring can be done in many ways, and this chapter aims to encompass most methods, drawing conclusions from multiple studies and establishing gaps in literature. A variety 
of techniques are described in this literature review including pyrometers, cameras (visual, NearInfrared (NIR) and Infrared (IR)), x-rays, incoherent imaging and laser profilers. The integration of these monitoring techniques into AM processes has shown positive results in improving process understanding, build quality, microstructure, mechanical properties, and reducing defects.

\subsubsection{Pyrometry}

Pyrometric devices have been used to measure melt pool surface temperatures to develop a better understanding of AM processes and/or to create control systems, subsequently improving clad quality [13-19]. M. Islam et al. combined a Complementary Metal-Oxide Semiconductor (CMOS) camera and a pyrometer to improve build quality in PBF techniques [17]. The authors used the pyrometer to monitor parameters and successfully reduced the balling phenomena in the PBF process through enhanced parametric understandings.

Furumoto et al. combined pyrometry measurements with a high-speed video camera to measure surface temperatures of the melt pool and record the consolidation phenomena in a PBF process. This system was used in multiple studies to provide a better understanding of the PBF process, allowing the authors to address defect issues such as shrinkage [18, 20, 21].

A. Nassar et al. implemented a two-colour pyrometer into a DED system to develop a closed loop control feedback system that altered the deposition path and hatch spacing to counteract heat accumulation in certain areas of the build [19].

The accuracy of liquid surface temperature measurements using two-colour pyrometers has been reported in detail by Monier et al [22]. The authors successfully used a two-colour pyrometry system consisting of two identical IR cameras. They determined that the designed system can accurately measure the surface temperature of liquid metals for range of materials, and stated that measurements are not effected by chemical composition. This said, their concluding remarks state that the surrounding environment to the subject under measurement can influence the accuracy of measurements. They state that fumes and reflections are likely to cause variations in measurements, both of which are likely to occur in AM processes.

There are different methods of measuring surface temperatures of metals, but two-colour pyrometry 
is the most popular method used in AM. Two colour pyrometry has been successfully used in AM to measure surface temperatures, improve process understanding and create control systems [18-21]. This has subsequently led to the improvement of clad quality and the reduction of defects in AM.

\subsubsection{Cameras}

\subsubsection{Camera Setups and System Architectures}

A range of studies have used cameras in AM processes to monitor a variety of parameters including melt pool dimensions, powder distribution, melt pool signatures, melt pool signals, melt pool splutters and cooling profiles [10, 17, 23-32, 32-61]. Meriaudeau et al. integrated multiple Charged-Couple Device (CCD) cameras into build chambers to distinguish what the key process variables are in AM process [23-25]. They studied the effects of changing variables on final component quality. Measuring parameters throughout AM builds allows users to subjectively change process variables to improve part quality. The trend of identifying key variables in AM processes to create better process understanding and to improve part quality is documented in the following research publications [23-26]. There are over fifty variables in AM processes that are known to effect the final component [3], but reviewing multiple parametric studies it is generally accepted that laser power and deposition speed are the two major parameter settings in AM processes. PMFR is another major parameter in specifically powder blown DED processes.

The installation of cameras into AM processes to monitor parameters throughout build cycles can be done in multiple ways. Hu et al. installed a NIR camera system to monitor melt pool geometry in a coaxial manner, and used a Canny edge detection algorithm to identify the edges of the melt pool [27]. The installation of cameras in a coaxial manner is something that is seen throughout many documented melt pool monitoring systems [10, 26-33, 43-46].

Asselin et al. produced a trinocular camera system that used three cameras equally spaced around the laser cladding head [34]. This system captured images from two out of three cameras depending on the angle of the melt pool. They ran similar image processing techniques on the image feeds using a binarization threshold. Their system is depicted in Figure 5 .

Kruth et al. published multiple papers using a system that combined a CMOS camera with a 


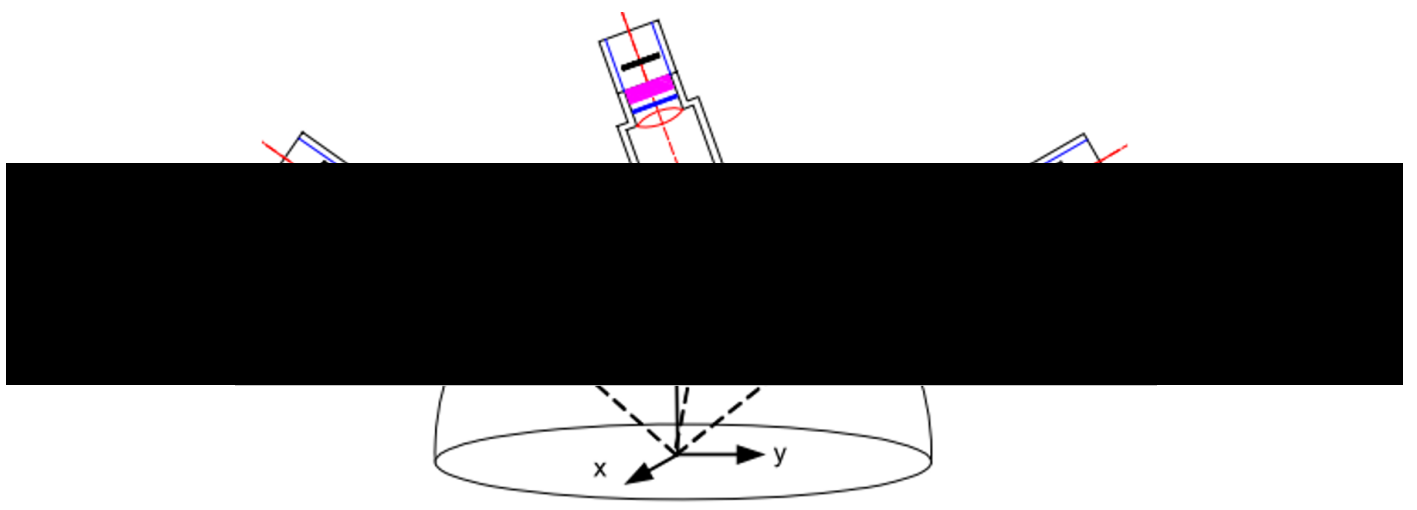

Figure 5: A schematic representation of the trinocular set-up used to perform melt pool measurements in experiments carried out by Asselin et al. [34].

photodiode [10, 28-33]. Both sensors were set-up in a coaxial manner to monitor the melt pool throughout PBF processes. The photodiode in the system is used to integrate all the recorded radiation, whilst the camera is used to extract melt pool geometry. A schematic of their system is detailed in Figure 6.

Craeghs et al. (previously referred to as Kruth et al.) further developed this system by implementing an off-axis camera to monitor powder distribution between different build layers in the PBF process [10]. The authors implemented multiple lighting sources from different directions and were able to identify uneven powder distributions caused by coater blade damage. The off-axis camera schematic is depicted in Figure 7.

This off axis camera position has both advantages and disadvantages. A major advantage is that there is less complications in the installation process when implementing them into PBF machines. Many off-axis cameras used in PBF processes are installed outside the build chamber meaning PBF machines do not need to be modified. These cameras must consider the transmission of specific electromagnetic frequencies through the viewing windows and often must undergo extra image processing steps to translate the angled camera view into a birds-eye perspective, to represent an image like that experienced through a coaxial view. Using cameras in this fashion has given rise to diverse monitoring 


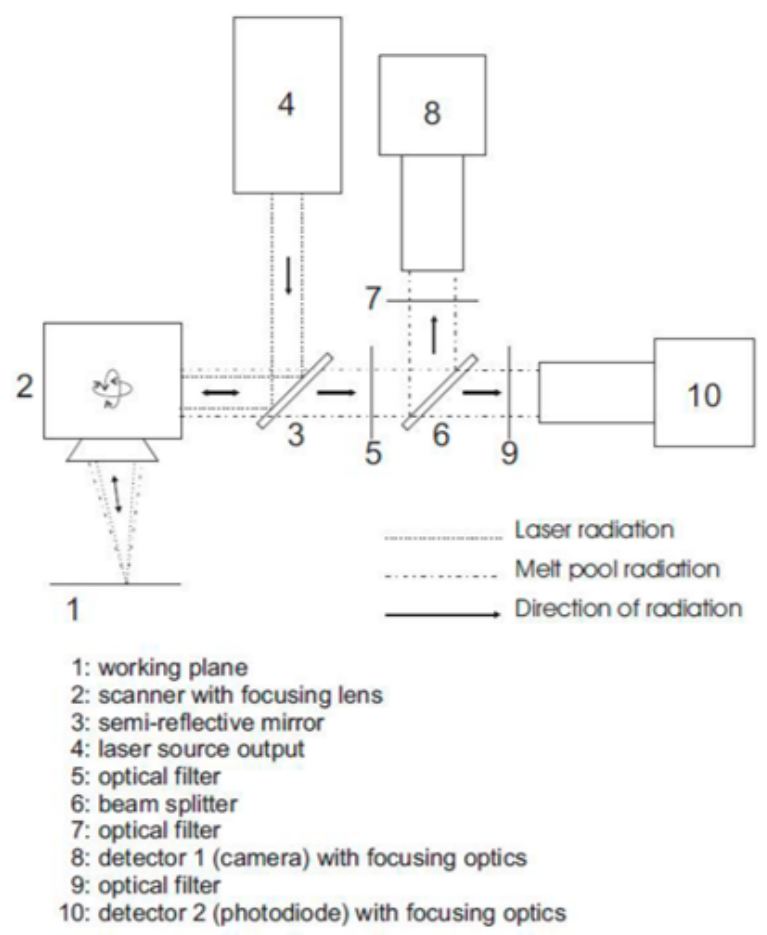

Figure 6: A schematic representation of the CMOS-photodiode parameter monitoring systems used by Kruth et al. [30].

techniques.

Klenzczski et al. used an off-axis camera set-up to develop automatic image processing techniques that detect dimensional accuracy and powder distribution properties $[35,36]$. This was again revisited by Scime and Beuth who designed and compared multiple Machine Learning (ML) techniques to determine which performed best in detecting powder distribution defects [37, 38].

Rodriguez et al. implemented an off-axis IR cameras into an EBM system to monitor parameters throughout build cycles, firstly using a subjective decision made by an operator to approve build quality [39]. The authors then subsequently developed image processing techniques to automate this process [40], introducing a technique to automatically detect geometry deviations and porous regions during builds. This research group used their developed parameter monitoring technique to identify porosity in-situ and perform corrective actions through laser re-melting [41, 42]. 


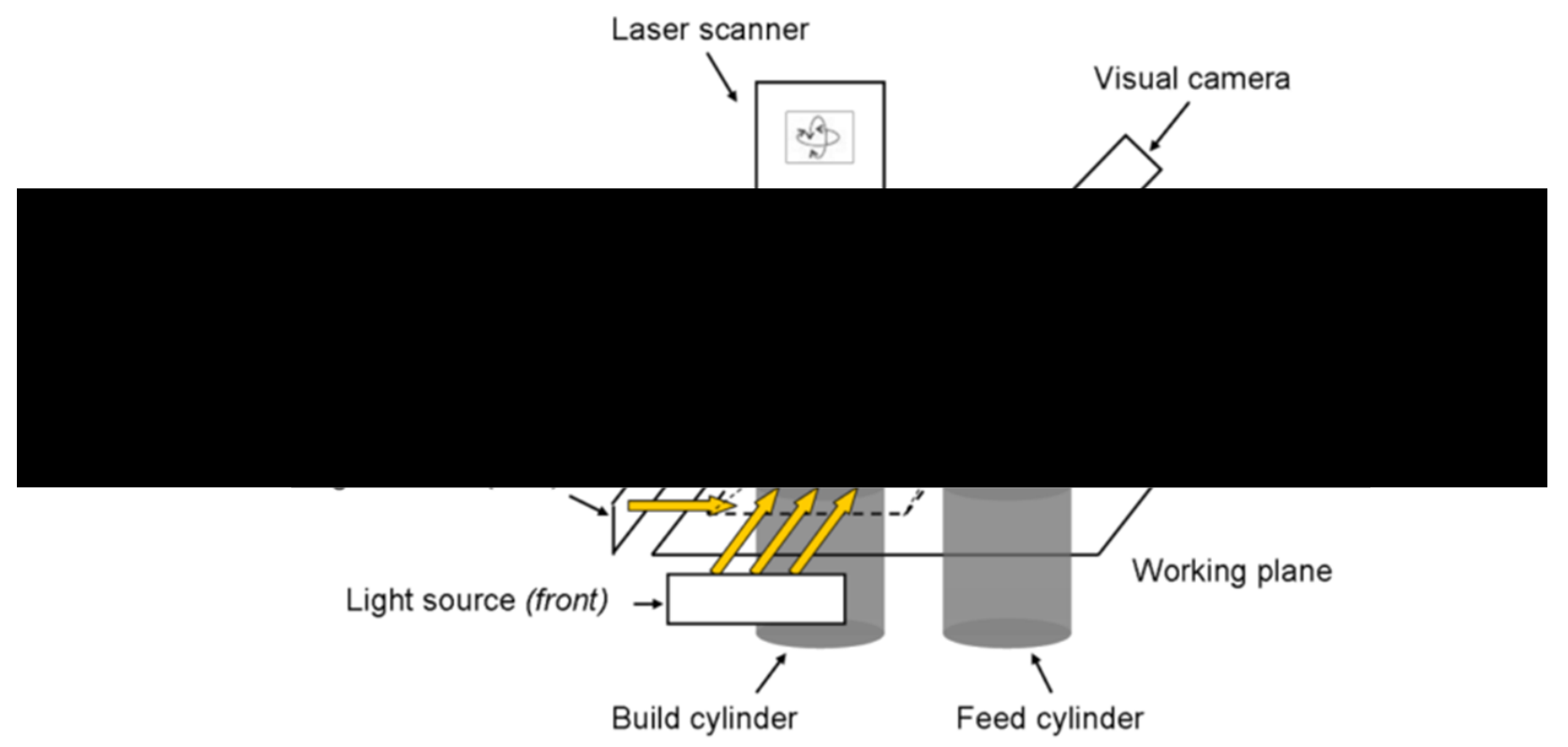

Figure 7: A schematic representation of the off axis powder bed monitoring system used by Craeghs et al. $[30]$.

\subsubsection{Melt Event Monitoring}

There are a range of different parameters within AM processes that have been monitored but a substantial amount of research has focused on capturing and measuring data surrounding the melt pool phenomena [10, 13-17, 17-19, 23-34, 43-58]. The melt pool phenomena is an phase in the AM process where energy meets material and a molten metal pool is formed. The melt pool phenomena is the first instance where key variables interact with each other and monitoring this can provide in-situ information about the build. The melt pool can provide an abundance of information about the quality of deposition and has been used on occasions to optimise parameters, develop control systems and detect defects.

An abundance of literature details the use of visual, NIR, IR and/or thermal cameras to monitor the melt pool phenomena $[10,17,23-34,43-58]$, with a certain literature focusing on specifically melt pool dimensions [10, 27-33, 43-47].

Colodrón installed a coaxial CMOS camera into a DED process to monitor melt pool behaviour 
and applied a threshold value on the image feed before calculating the melt pool width [43, 44]. The width was calculated by measuring the first row that was populated by a white pixel, and the last row populated by a white pixel. The image processing technique was later refined by Araujo et al., and an elliptical approximation was super imposed on the melt pool using moments of the binary image [45]. Calculated melt pool widths of the described image processing techniques are displayed in Figure 8. Hofman et al. used a similar technique to successfully reduce the effects of dilution in a DED process [46].

Clijsters et al. attempted to calculate melt pool dimensions using a NIR camera feed [32]. Their image processing technique corelates a pixel intensity value with the solid-liquidus interface of the melt pool before binarising the image. The width and length of the melt pool is then calculated by calculating the maximum pixel counts in directions parallel and perpendicular to the scanning direction respectively.

Cheng et al. used a NIR thermal imaging camera to record thermal images of the SLM process throughout build cycles [47]. The authors developed a new melt pool dimensioning technique that uses a single emissivity value based on the pixel intensity at a discontinuity located at the liquidus-solidus transition point. This pixel intensity value was used as a binary threshold to allow for the extraction of melt pool dimensions.

Multiple research papers have eradicated the measurement of melt pool dimensions in their system and have instead introduced techniques that measure melting event signals, splatters, and/or signatures throughout AM builds [48-58]. The premise of this technique is that different melting event signals are shown depending on the condition of the melt event. These can be used to predict build quality.

Ye et al. investigated plume and splatter signatures using an NIR camera mounted externally to the build chamber [48]. In their study they varied laser power and scanning speed settings to introduce overheating and underheating conditions - producing defects such as balling. They were able to correlate certain poor conditions to changes in plume and splatter signatures.

Zhang et al. used a high speed camera to monitor melt pool intensity, plume area, plume orientation, spatter number, spatter area, spatter orientation, and spatter velocity [49]. The authors used image processing techniques to extract features and discovered that extracted features are related to 


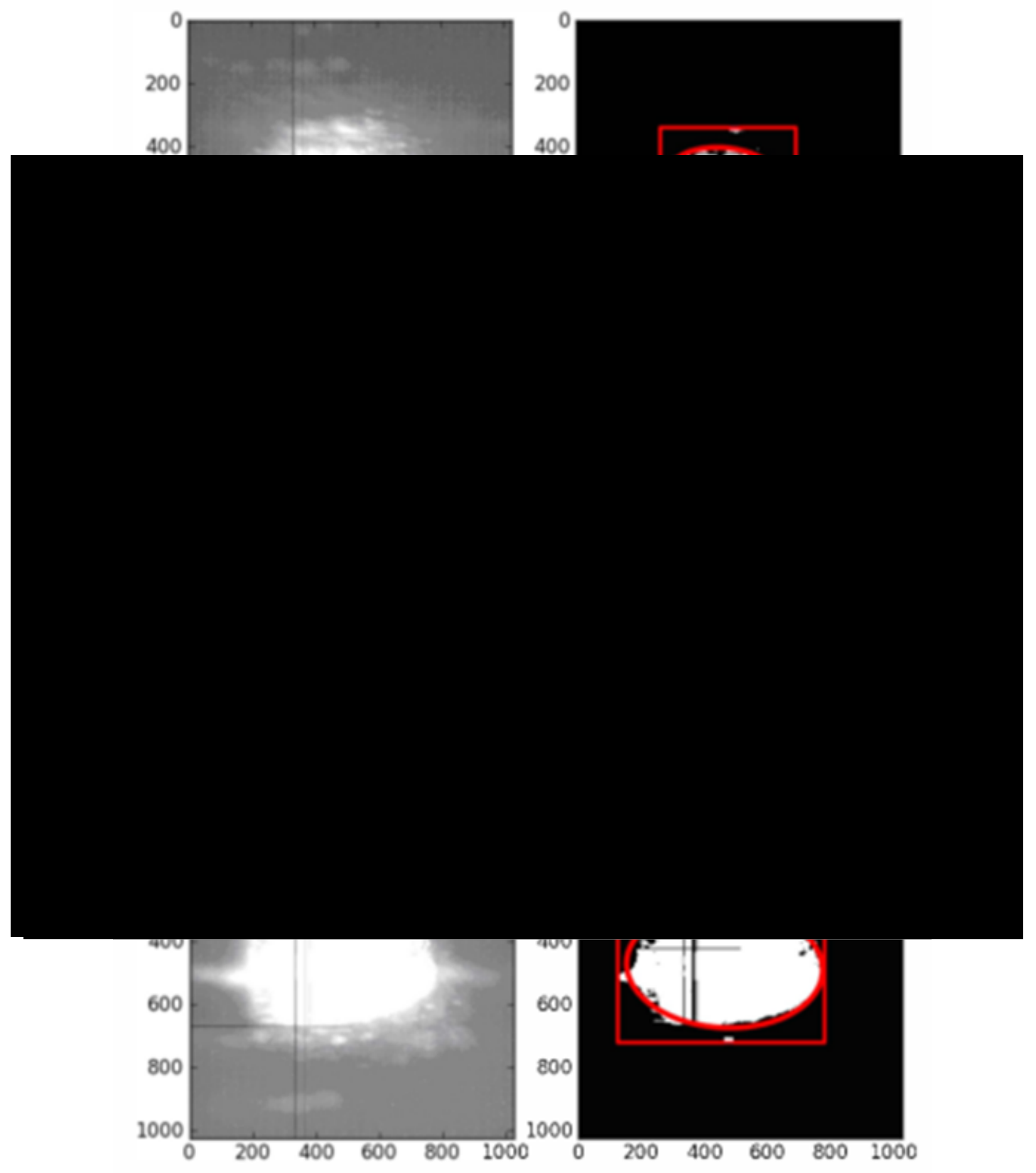

Figure 8: Image processing calculations of melt pool width using both the old (box) and new (ellipse) algorithms on three separate occasions [45]. 
energy input, melt pool status and the formation of defects. Multiple studies have correlated melt pool event signatures to defects or poor melt conditions in built components [48-54], with others using these techniques to predict mechanical properties [55]. Kolb et al. used this technique to develop cavity cleaning methods and to improve the quality overhang structures [56].

Correlating melt pool signatures to clad quality and defect locations can be done using ML $[51,52$, 57, 58]. Zhang et al. compared two intelligent classification methods (Convolutional Neural Network $(\mathrm{CNN})$ and Support Vector Machine (SVM) techniques) to determine their ability to identify anomalies during PBF processes [58]. The CNN methods trained with raw data outperformed the SVM methods trained with extracted features in identifying anomalies.

Khanzadeh et al. used ML to develop a method of being able to monitor defects in real time to allow them to control parameters throughout a DED process [51]. Scime and Beuth conducted a similar study in which they implemented the Bag of Words (BoW) ML technique in combination with Scale Invariant Feature Transform (SIFT) to counteract the varying sizes of the melt pool experienced in PBF processes [52]. Their work allowed them to correlate data captured from a high-speed camera to defects, although their conclusion states uncertainty in the exact relation.

Ye et al. determined Deep Belief Networks (DBN) to be more accurate than both Multi-Layer Perception (MLP) and CNN ML techniques in their research to recognise melt states at various energy densities [57].

The discussed academic advancements are being propelled into industrial applications and commercial products. Concept Laser, a General Electric company, have introduced a range of process monitoring systems with one of them being a melt pool monitoring system [62,63]. The "QM module meltpool 3D" uses coaxial sensors to detect melt pool emissions that are created during the process. Signals emitted from the melt pool are captured and used to monitor melt pool size and intensity. The commercialisation of these products shows that melt event monitoring technique are valid for industrial applications and improving the academic research surrounding these systems will likely have positive effects on industrial applications. 


\subsubsection{Cooling Profiles}

The solidification stage (cooling profile) of the melt pool has also received research dedication [59-61]. Hooper developed and used a two-wavelength imaging system to motor cooling profiles throughout a PBF process to better understand process fundamentals [59].

Zalameda et al. used a NIR camera to image both the melt pool and its solidification period in an EBF system [60]. They measured the transient cooldown phase of the melt pool and were able to use these measurements as a method of Non-Destructive Evaluation (NDE). They report that defects can be detected by measuring the cooldown phase but suggest that this conclusion requires further results.

Farshidianfar et al. combined an off-axis IR camera with a CCD camera to create an imaging system to monitor cooling profiles throughout a DED process [61]. The authors implemented a closed loop feedback control and were able to create desired microstructure by selecting a cooling rate and regulating it with feedback control.

\subsubsection{Other}

Guo et al. developed a system to allow for x-ray imaging of a PBF process to study the dynamics of powder spattering as a function of time [64]. The set-up is depicted in Figure 9. Using this set-up the authors were able to understand the powder splattering phenomena in detail and determined that the process could be optimised by pre-sintering and tuning both layer thickness and environment pressures.

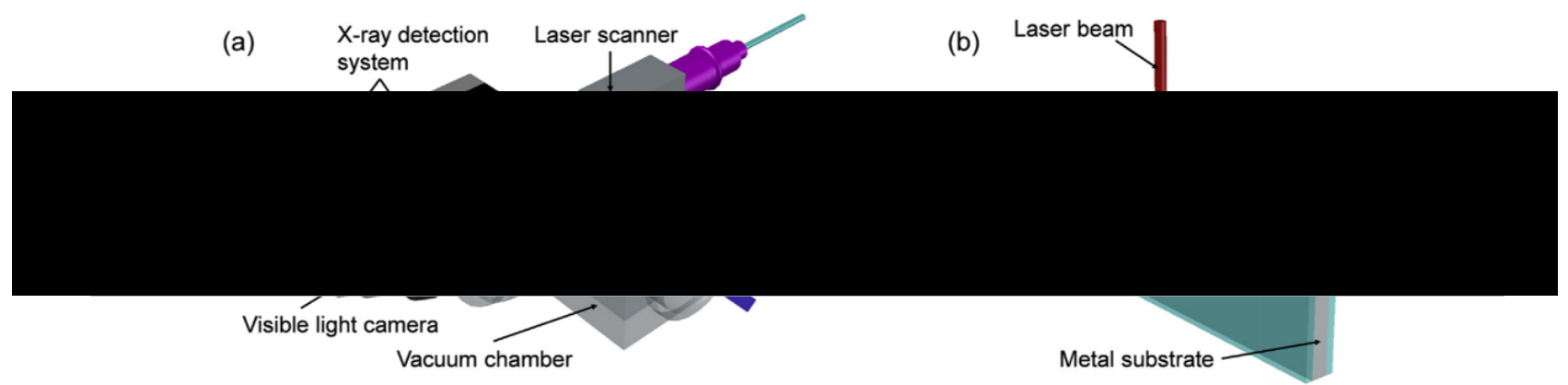

Figure 9: A schematic representing the X-ray imaging systems used to study the dynamics of powder spattering in PBF processes. (a - entire system. b - close up) [64]. 
Kanko et al. developed a in-situ defect detection system using inline coherent imaging to monitor a PBF process [65]. The authors used the technique to provide a more detailed understanding of the PBF process, and state that they were able to identify different material phases, defect locations and poor parameter spaces.

A. Heralić et al. utilised a 3D laser profiler, CMOS camera and oxygen monitor to create a closed loop parameter monitoring system in a wire DED process [66]. The laser profile was used after each full deposited layer and parameters were measured throughout the process. Parameters were altered in-situ to successfully compensate for height deviations measured by the laser profilers. Figure 10 displays the system they developed.

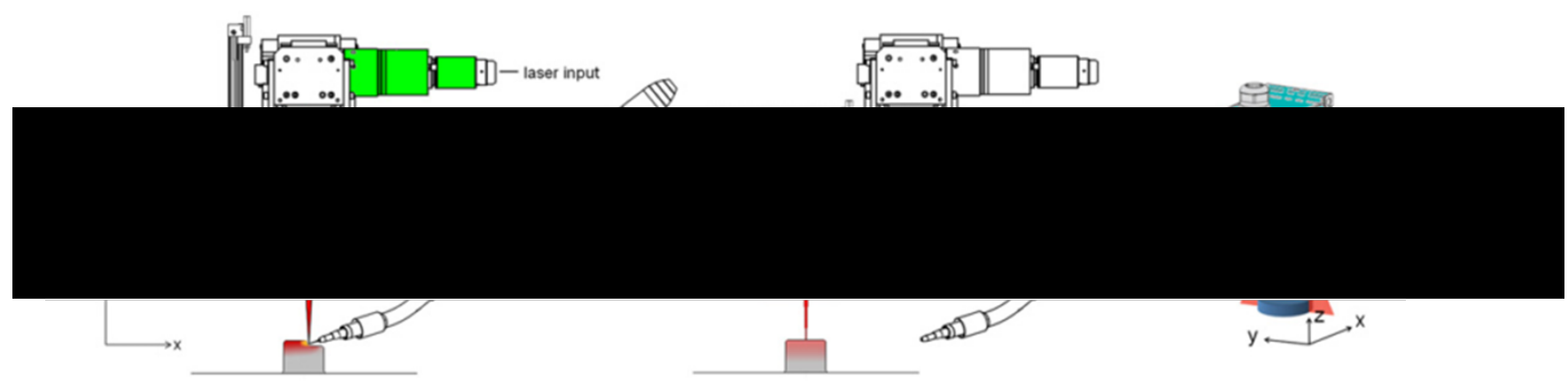

Figure 10: Diagrams representing: The deposition mode of a single layer (Left), the scanning mode after the production of a single layer (Middle), and the coordinate translations during scanning with axis reference (Right) [66].

\subsubsection{Conclusion}

The compilation of literature surrounding parameter monitoring systems in AM has highlighted the benefits of installing them. Installing parameter monitoring systems in to AM machinery grants the user a better understanding of the complex AM process, and can be used to optimise parameter settings, build control systems and detect defects. Control systems and parameter optimisations have been documented in many research papers to directly result in improved quality of AM built components $[10,15-17,19,28-31,33,35,36,39,40,43-46]$. The majority of parameter monitoring systems can be broken down into either pyrometric or camera-based parameter monitoring systems. 
Both systems have allowed for increased understanding of AM processes and subsequently allowed for improved deposition in both PBF and DED systems. Both have seen their use in the development of control systems $[10,15,19,23-25,27-31,33-35,39,40,43-46,67]$.

Cameras have been used to calculate surface temperatures of the melt pool by correlating emissivity values with radiation intensity [10, 23-25, 28-31, 33, 39, 40, 43-46, 68]. This means that they can perform the same measurements as pyrometric techniques but have the added advantage of a high-resolution pixel array. Due to this factor, the focus of this research will look to explore the benefits of camera-based melt pool monitoring systems by furthering the research outlined by previous literature. Camera based systems have been used to monitor powder bed distributions, perform melt pool dimensioning, and observe melt pool splutters, signatures and signals [10, 17, 23-32, 32-58]. This research aims to develop DED systems, meaning that the use of camera systems to monitor powder bed distributions cannot be explored. Whilst event signals and splutters have shown to provide key information about AM processes all related references have been used in PBF systems. Due to the proven implementation of melt pool dimensioning in DED systems [43-46] the focus of this research will be that of melt pool dimensioning.

\subsubsection{State of Knowledge and Literature Gaps}

The state-of-the-art techniques show limitations in the dimensioning of the melt pool for two major reasons:

\subsubsection{Low Quality Melt Pool Images}

Most documented images of melt pools are of low quality, and lack detail about the complex interaction between metal powder, substrate and laser power [27, 34, 45, 46, 69]. Figure 11 shows examples of melt pool images that have been used in the development of control systems, all of which show high levels of saturation.

Saturation of melt pool images can lead to incorrect melt pool measurements. In these images there are no distinct features that provide an indication to a melt pool edge. If a melt pool image contains a large proportion of pure white pixels in it, then there is a high possibility that the user is 

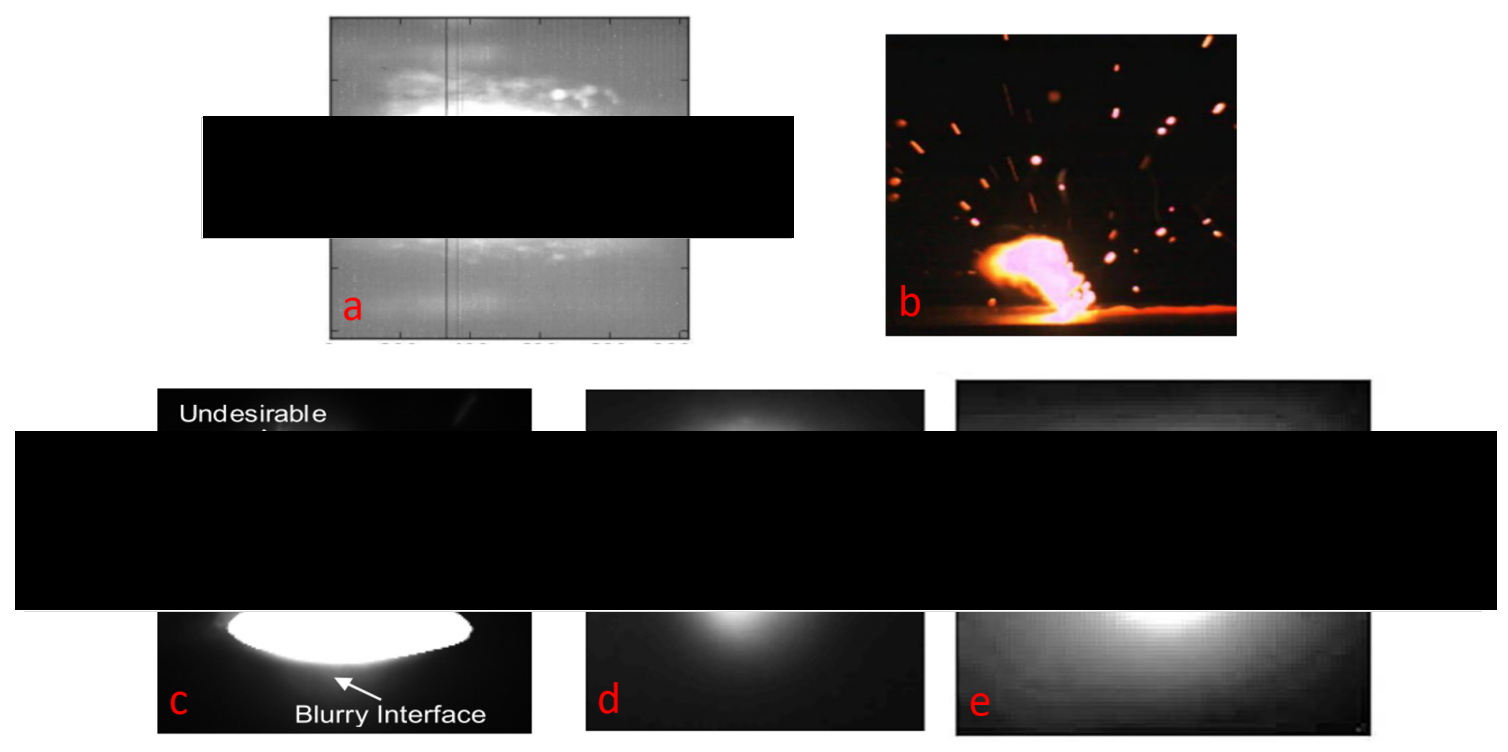

Figure 11: Current state of the art melt pool images: a. [45], b. [69], c. [34], d. [46], e. [27].

not only measuring the molten metal pool, but also the surrounding substrate.

\subsubsection{Emissivity Based Dimensioning Techniques}

Throughout the literature, multiple papers have quoted inaccuracies when using emissivity values in melt pool dimension calculations [39, 40, 47]. Emissivity is a value that is material, angle, and surface dependent, meaning the calculation of such a value for a molten metal is extremely difficult. The melt pool in AM processes is constantly changing material, shape and surface characteristics meaning that emissivity would change throughout deposition. The current state of the art systems use constant emissivity values in the calculation of melt pool width throughout AM builds and do not take into consideration emissivity fluctuations[10, 23-25, 27-31, 33, 34, 39, 40, 43-47, 67].

\subsubsection{Final Review}

The literature has highlighted the key benefits of using melt pool dimensioning systems in AM processes, but has also highlighted some of the current limitations. This work will aim to optimise machine 
vision techniques used for melt pool monitoring in DED processes to provide higher quality imaging of the melt pool. Subsequent work will then use acquired high-quality images to design an improved algorithm that can measure melt pool geometry without the need for emissivity values. These techniques will then be used to better understand the complex parameter interaction in DED processes and allow for the direct comparison between different materials. 


\section{Machine Vision in Laser Metal Deposition Processes}

Current literature cites the use of machine vision techniques as a method of monitoring AM processes. The use of image sensors has allowed for non-contact measurements in harsh environments, and their utility in the field has already proved incredibly valuable.

In the previous chapter, the evolution of image sensing devices used for monitoring AM processes was discussed. This chapter outlines the core concepts of how they work, the effects that optical components have on acquired images, the effects that settings have on acquired images, the limitations that are present when using image sensors in AM systems, and how changing exposure time can allow for high quality melt pool imagery at a variety of laser powers.

This chapter optimises machine vision imaging techniques and produces high quality melt pool images with edge features. This chapter explains the importance of exposure time in melt pool imaging, and documents an equation that can be used to achieve high quality melt pool images at a range of different laser power settings.

\subsection{Components of a Camera}

There are two main components in a camera that are to be briefly discussed. The image sensor is the most fundamental component of a camera - and absorbs EM radiation, converting it into electronic signals. These components however will not be able to accurately form high quality images without help from optics within the camera. Combining these machine vision fundamentals together with a solid understanding of radiation mechanics allows for optimised melt pool imagery.

\subsubsection{Image sensors}

Image sensors are devices that can detect information across the EM spectrum, and work by converting photons into electrical signals. Image sensors have different quantum efficiencies depending on their application and can detect wavelengths from radio waves (used in radar and sonar), through to x-rays 
(used in medical and NDT environments). This research concentrates on the part of the EM spectrum which contains thermal radiation, and especially wavelengths within the band of NIR radiation.

\subsubsection{Visible Light Sensors}

Most image sensors that are on the market today are manufactured to have a high functionality within the visible light spectrum. Due to higher user demand of image sensors within this spectrum, more research and capital investment has been directed to the manufacturing of these photoelectric devices. There are two main visible light image sensor architecture types that are commercially available, CCD and CMOS. These architectures have competed for years, and over time have both been regarded as the flagship sensory device.

CCD image sensors undergo a manufacturing process that allows them to transport charge, generated by incident light, across the chip to be read from a single point. This results in a sensor that has fidelity and high light sensitivity. CCD sensors are known for their high-quality, low noise images, but a disadvantage is that they tend to draw more power and are more expensive [70].

CMOS image sensors undergo the same manufacturing process experienced by most microprocessors, and read off electrical charge differently to a CCD sensor. Each pixel on a CMOS sensor has a built in capacitor and amplifier, meaning that the pixels are read in parallel. CMOS sensors have the advantages of lower power consumption and faster image acquisition capabilities. Their disadvantages however, are that they subject to higher levels of noise [70].

At this present moment, CMOS sensors dominate the market in machine vision applications due to reduced power consumption, cheaper manufacturing costs, and faster acquisition rates. Some applications still use CCD sensors, and they do still have their advantages, but the advantages of using CMOS sensors result in a more prominent use of them in the modern market [71].

\subsubsection{Infrared Sensors}

Infrared (IR) sensor architecture is more complex than that of visible light sensors, and over the years a range of different detectors have been developed. The IR region of the EM spectrum spans from $700 \mathrm{~nm}$ to $1,000,000 \mathrm{~nm}$ ( or $1 \mathrm{~mm}$ ), which covers 3000 times more wavelengths than that of the visible 
spectrum. This range of wavelengths requires different types of sensors for different viewing purposes. Different materials are used for their quantum efficiencies at specific wavelengths [72].

IR detectors can be categorised into two main types, thermal and photon. Thermal detectors are sensors that absorb incident energy and warm up. This in turn changes the electrical resistance of the component, which can be measured and used to provide a graphical representation of EM radiation.

Photon detectors are like image sensors in the visible light spectrum. These detectors absorb photons and produce free electrical carriers as a result - much the same as visible light photons on a silicon chip. These electrical carriers can then be measured, and varying intensities across different pixels can be used to represent an image. These detectors have a quick response time, but often require cooling to eradicate dark currents that cloud the image. These types of detectors include; photovoltaic, photoconductive, photo-emissive and Quantum Well Infrared Photodectors (QWIPs). The materials used for these sensors change depending on the wavelengths that they are designed to observe. Materials include lead sulphide, lead selenide, mercury cadmium telluride and indium gallium arsenide. The list of materials that have been used for different IR sensors is vast. The combination of reduced target market and complex materials results in IR imaging devices being more expensive when compared to their silicon counter parts [73].

\subsubsection{Near-Infrared Sensors}

The majority of NIR sensors are silicon based. Silicon's quantum efficiency is sensitive to wavelengths up to around $1100 \mathrm{~nm}$. Above this wavelength silicon is transparent and allows photons to travel through the material, similar in the way light passes through glass [74]. This means that radiation in the region of 700 to $1100 \mathrm{~nm}$ can be detected through the use of normal silicon-based CMOS and CCD sensors. Though this is true, the quantum efficiency of silicon at wavelengths above $700 \mathrm{~nm}$ is reduced compared to that of wavelengths within the visible light spectrum. Emissions that are dominant in this region can still be detected by enhancing the quantum efficiency of the sensor in this region. This greatly increases the sensitivity to NIR wavelengths [74].

This work focuses on viewing molten metal in AM processes. Peak wavelengths at the temperatures of molten metal reside in the NIR region, therefore making NIR devices suitable for melt pool imagery 
[32]. Working with NIR cameras gives the added advantages of CMOS and CCD technologies including reduced cost, larger pixel array sizes and the absence of active cooling.

\subsubsection{Optics}

Optics in imaging systems are incredibly important. Without the correct optics, the digital representation of EM radiation can be unfocused, contain aberrations, and have radiation from undesired wavelengths. Optics in imaging devices include focusing optics and filtering optics.

Focusing optics in cameras are used to direct EM radiation from the Filed Of View (FOV) onto a small image sensor that is contained in the main body of the device. The material used for the focusing optics is dictated by the EM radiation that the user desires to capture. Whist the image sensor should have a high quantum efficiency across desired wavelengths for optimum sensitivity, the focusing optics used should have little to no interaction with radiation of these wavelengths.

The material's properties dictate the absorption of EM radiation and thus determine whether the given material is transparent for certain wavelengths. A material is transparent to certain wavelengths when photons with those wavelengths are not absorbed. A photon is absorbed in a material when an interacting photon provides the correct energy to excite an electron to a higher energy state. Certain materials do not respond to certain wavelengths because these do not provide the correct energy to cause electrons in the material to cross the band gap. Electromagnetic radiation can also interact with matter to cause changes in vibrational and rotational energy, with bond stretching being an absorption mechanism for IR radiation [75].

Optics that are used for image capture in the visible light spectrum can be used in the NIR spectrum due to similar wavelengths. Again, this provides a lower cost system compared to cameras that operate in the mid and far IR regions, as expensive optics do not need to be purchased [76]. The transmission of focusing optics should be high for the desired wavelengths of capture.

Filtering optics are used in digital imaging to stop certain wavelengths of light reaching the image sensor. In most modern DSLR cameras, there are NIR filters that block unwanted IR radiation and alter visible light images [71]. Types of optical filters include bandpass, longpass, shortpass, colour 
and neutral density. Filters can be used independently or in combination to block out wavelengths that the user does not want to measure [77].

Combining both focusing and filtering optics together can allow for a much higher image quality for melt pool monitoring systems. Using this technology, the high intensity and large range of EM radiation can be controlled to provide high quality images of the melt pool.

\subsection{Camera Settings}

There are various settings on machine vision cameras that can be manipulated to get both higher quality images and higher frame rates. Optimising these settings, once a camera is installed into an AM machine, is something that can drastically improve the performance of the melt pool monitoring system. This section outlines the settings that have a noticeable effect on the AM monitoring system performance.

\subsubsection{Shutter Mode}

There are two types of shutter modes in modern imaging, global and rolling. Most CCD sensors employ global shutters, and inherently CMOS sensors have rolling shutters. A CCD device works by opening the shutter, capturing EM radiation, closing the shutter, and then reading the individual pixels in a serial pattern. This means that all pixels are exposed to the same instance of EM radiation for the same duration, giving geometrically stable images. CMOS technologies differ in the way that they capture EM radiation. CMOS sensors read EM radiation line by line - usually starting from the top of the sensor and moving towards the bottom. This causes a slight variation in time between the readouts of each individual row in the height of an image. Due to this rolling shutter action, images that are acquired can exhibit the "jello effect" [78]. Modern technology has allowed for the use of global shutters on CMOS sensors. This read out pattern requires a more complex design to the CMOS sensor, but has the added advantage of reduced image distortion, whilst retaining the other benefits of using CMOS sensors. The camera selected for melt pool imaging uses a global shutter to reduce image distortion. 


\subsubsection{Area of Interest}

The majority of modern machine vision cameras have an Area of Interest (AOI) feature, also referred to as a Region of Interest (ROI). This self-explanatory name selects an area of an image for focus, and only processes the pixels that are within it. For many machine vision applications, only small parts of an image are required to perform image processing on, and reducing the captured images consequently reduces the amount of processing that the camera must perform. Reducing the processing requirements of the camera results in a higher maximum frame rate. Certain manufactures provide solutions with multiple AOI capabilities. An AOI is used in the developed melt pool monitoring system to increase the cameras frame rate.

\subsubsection{Frame Rate}

A setting that ties in with AOI's is the cameras frame rate. The frame rate of a camera dictates how many frames are taken within a second. Having a high frame rate can allow for a deep understanding of fast processes, and certain machine vision applications require high-speed cameras. The frame rate requirement for a system is dependent on the users' needs. High frame rate cameras are more expensive than their normal counterparts - and computationally expensive image processing techniques can cause bottlenecks soon after frame acquisitions if they are not optimised. Having a high frame rate camera can provide a lot of information about a system, but if the frames are not properly utilised, the user is not taking advantage of the extra overhead cost.

\subsubsection{Exposure Time}

Controlling of the shutter speed/exposure time of image sensors is very important for acquiring high quality images. The exposure time is the amount of time that the shutter of the camera is open, allowing EM radiation onto to the sensor. Increasing the exposure time settings can allow for more radiation to be absorbed by the sensor, thus providing more information. Having an exposure time too high can however saturate the image and take away valuable information by flooding the sensor

with EM radiation. As well as causing image saturation, having a longer exposure can cause image 
distortion, as faster moving objects will appear to stretch across the screen. An example of this effect is pictured in Figure 12 .

Selecting a correct exposure time can give optimum image quality and provide critical information for melt pool monitoring systems. The exposure setting of the camera has proven to be an effective tool in acquiring high quality melt pool images at a range of different laser power settings.

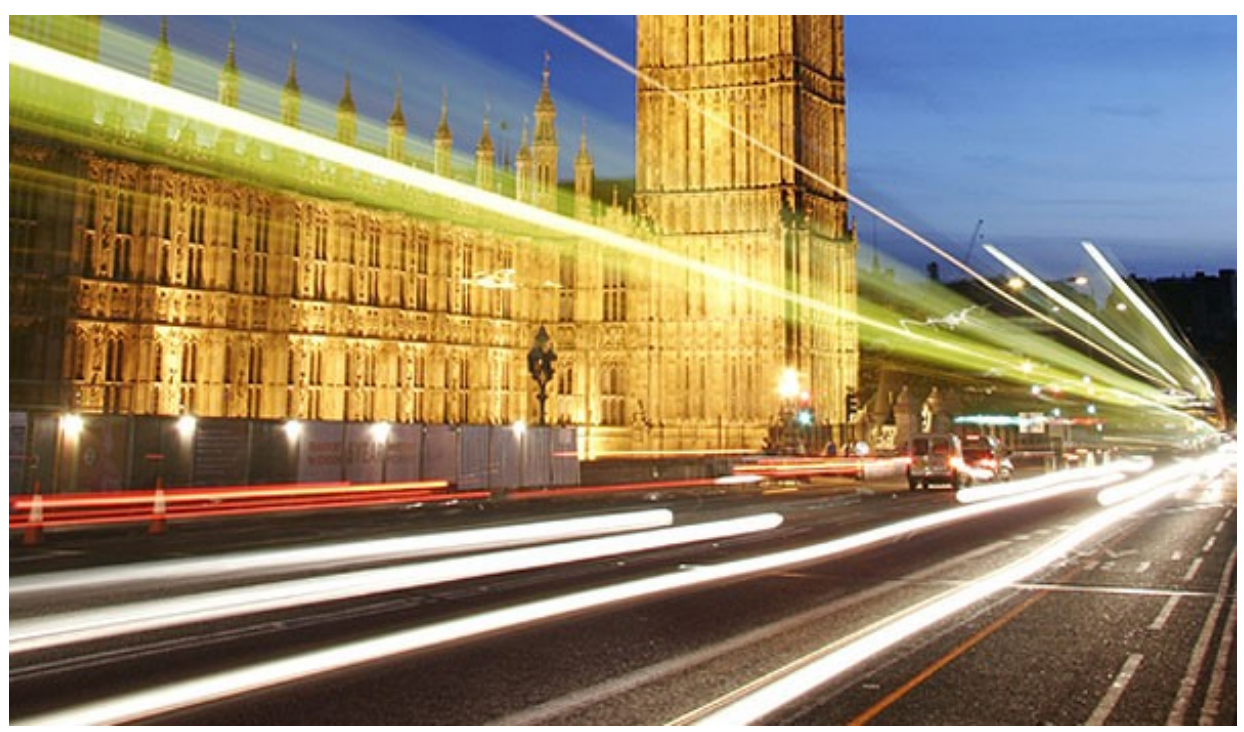

Figure 12: An image displaying the effects of long exposure times on moving objects [79].

\subsection{The Directional Emittance Phenomena}

Saturated melt pool images can lead to incorrect measurements of melt pool dimensions. Figure 11 (Chapter 2) displays melt pool images that have high levels of saturation. If a melt pool image contains a large proportion of pure white pixels in it, then there is a high possibility that the user is not only measuring the molten metal pool, but also the surrounding substrate.

Everything that has a temperature above absolute zero will emit thermal radiation. The region that contains thermal radiation extends from 0.1 - 1000 microns and includes a portion of the ultraviolet 
region, all visible and all IR radiation [73]. This means that the surrounding substrate will emit thermal radiation and will likely be emitting similar wavelengths and intensities due to it being in direct contact with the molten metal pool.

To add to these complications, emissions from vapour plumes that are above the surface of the melt pool can also contribute to the total thermal radiation. The following referenced paper states in their findings that an accurate melt pool width could not be extrapolated from their sensors for this very reason [80]. This plume can not only emit its own thermal radiation, but can also reflect, absorb, transmit, and scatter radiation that is transmitted by both the melt pool and the heated substrate. Powder particles that are blown into (or around) the melt pool can have a very similar effect on thermal radiation.

The calculation of melt pool emissivity to give accurate temperature readings is scientifically challenging. Emittance is the radiation mismatch between an object and a blackbody. Emissivity is the emittance of a particular material under certain conditions. The creation of a molten metal blackbody, to use for emissivity calibration, seems impossible in the physical world at this moment in time. Even if this was possible the emissivity value of a subject under measurement is dependent on both the material, and surface characteristics. These two things are rapidly changing in the complex interaction between blown metallic powder, laser heat input, and substrate throughout the AM process.

Even if the emissivity value of a molten metal pool could be calculated. A major influence of the emitted radiation is dependent on directional emittance. A surface that is not a true black body does not emit radiation at the same intensity in all directions. Emissions are at maximum intensity in the direction perpendicular to the surface and decrease to zero at angles parallel to the surface. This phenomena will be refereed to as the directional emittance phenomena throughout this thesis. Figure 13 displays the ideal blackbody radiation in conjunction with the real body radiation.

Using this theory, a melt pool whose contact angle is larger than approximately $60^{\circ}$, should transmit less detectable thermal radiation than the substrate surrounding the melt pool. However, this is not evident in any of the referenced melt pool images displayed in Figure 11.

A combination of all the issues stated above explains why it is a difficult task to view and measure the melt pool within AM processes. Without visually observing the droplet's surface, calculations of 


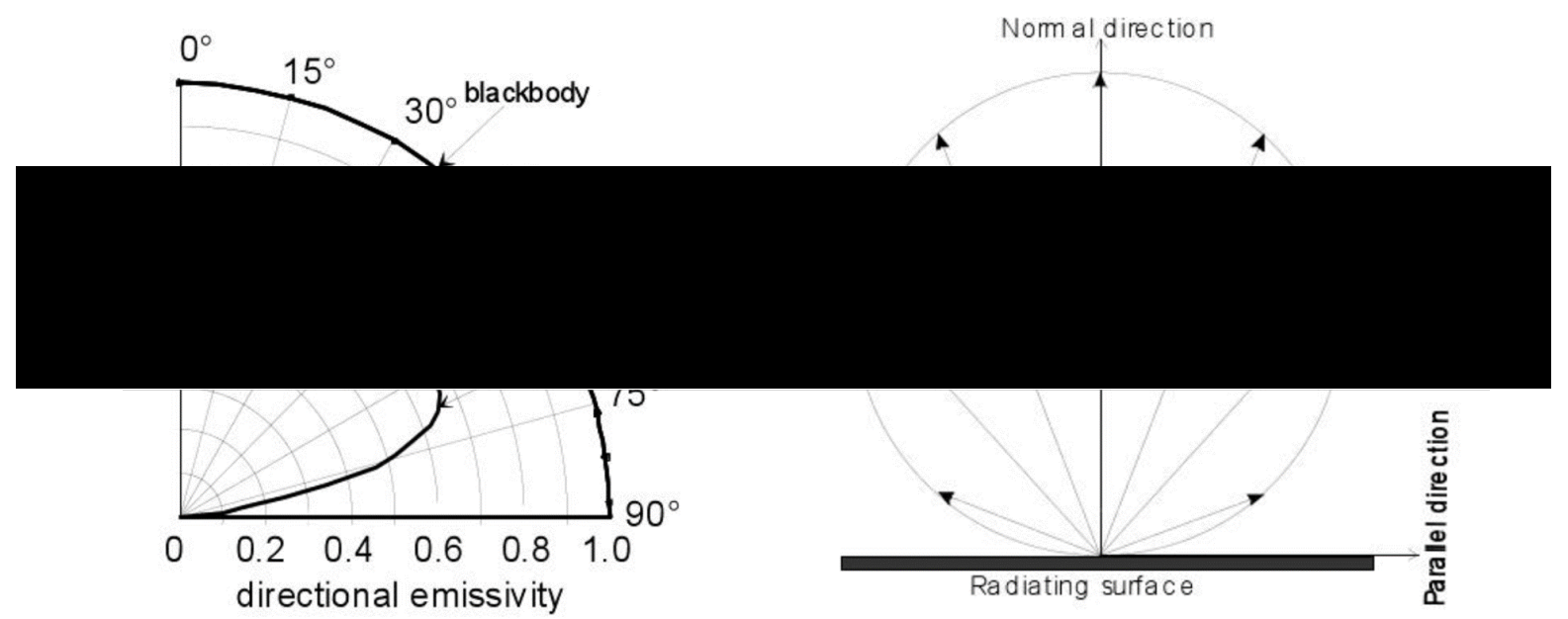

Figure 13: Left: A schematic representation of directional emittance from a flat surface. Right: Directional emittance of a blackbody and real body [73].

melt pool dimensions are not likely to be accurate.

Some notably high quality images have been observed in other AM processes, and these are displayed in Figure 14. The image on the left is taken using a standard high-speed CCD camera whilst the one on the right uses a CMOS camera with background illumination [81, 82]. Details about how these images are achieved are not noted in the referenced documentation [81, 82], although Lui et al. states the use of a very low shutter speed of $1 / 500$ s. It is also noted that both documents use wire deposition techniques, making it notably easier to view the melt pool, as stray powder particles do not inhibit the captured image.

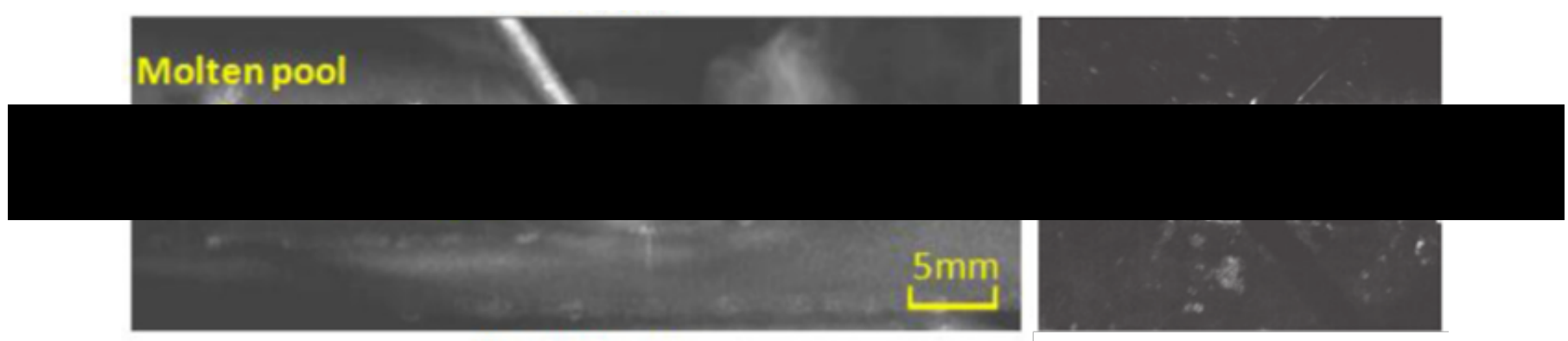

Figure 14: Left: A high quality melt pool image achieved using a high-speed CCD camera [81]. Right: A high quality melt pool image achieved using a high speed CMOS video camera [82]. 
The literature compiled in Chapter 2 provides a detailed analysis of different techniques used to monitor melt pools. The authors of [32] give a good outline on how to acquire quality images in an SLM process (although they do not publish melt pool images). They reference Planck's law and show the ideal radiation distributions for temperatures of 1400,1600 and $2000^{\circ} \mathrm{C}$. These radiation curves show peak wavelengths between 1500-2000nm, with the peak thermal radiation wavelength decreasing with increasing temperature. The author's state that the wavelength of the laser used is $1064 \mathrm{~nm}$, and state that their set-up cannot measure above this wavelength due to the CMOS quantum efficiency ceiling being $1000 \mathrm{~nm}$. The lower bound filter was set to $780 \mathrm{~nm}$ and the upper to $950 \mathrm{~nm}$.

Using this information in conjunction with past melt pool images and thermal radiation knowledge, viewing the melt pool in this region will likely give a clearer image of the melt pool due to radiation being of lower intensity. Capturing thermal radiation of the melt pool at peak wavelengths is challenging, as image sensors are more likely to become saturated.

\subsection{Experimental set-up for advanced melt pool imaging}

\subsubsection{Direct Energy Deposition Machine}

This research focused on the development of DED systems through improved melt pool monitoring and understanding. The DED system used was composed of a Trumpf Trudisk $80025.3 \mathrm{~kW}$ disc laser, a TruControl 1000 controller, a Trumpf BEO D70 processing optics with a motorised collimation laser deposition head, a Reis RV60-40 robot, a Reis RDKVv05 two axis manipulator and a Sulzer Metco $10-\mathrm{C}$ powder feeder with a dual $1.5 \mathrm{~kg}$ hopper arrangement.

The Trumpf BEO D70 processing optics has a module that allows for coaxial real-time viewing of the substrate and melt pool during deposition. The default camera installed on the device was used for laser/nozzle alignment prior to deposition. The camera is a Charged-Couple Device (CCD) camera that operates with high quantum efficiency in the visible light region, which produced fully saturated images when depositing. Internal optics in the laser deposition head filter out the wavelength of the laser light so that no damage is caused to the image sensor. Immediately after starting the cladding process, the image obtained from the coaxial CCD is completely saturated. The causation of this is the 
lack of filters allowing for thermal radiation to be transmitted onto the silicon chip. This abundance of radiation results in complete blindness to the AM process. The CCD camera can easily be removed using a set of Allen keys to allow for the installation of a new CMOS NIR camera. The old camera and exposed optical path are displayed in Figure 15.

\subsubsection{Camera}

Clijsters et al. provided the basic information for the purchasing of new optical equipment [32]. The information provided in their paper led to the purchasing of a NIR camera, that has a heightened sensitivity to EM radiation with wavelengths between 700 and $1000 \mathrm{~nm}$. The camera was purchased from a German company called IDS-imaging. The model of the camera is the U3-3240ML-NIR-GL CMOS camera. The camera has a monochromatic CMOS sensor and has an enhanced NIR response due to an increased pixel well depth [74]. This has the benefit of increasing sensitivity in the NIR region but has the disadvantage that there is some leaking of charge into surrounding pixels that reduces the resolution. The quantum efficiency response of the CMOS sensor is displayed in Figure 16. IDS-imaging base their camera design on sensors produced by $\mathrm{e} 2 \mathrm{v}$, and the sensor used in the NIR CMOS cameras is the EV76C661. More information regarding the camera can be found using the abundance of information on the IDS website [74]. 

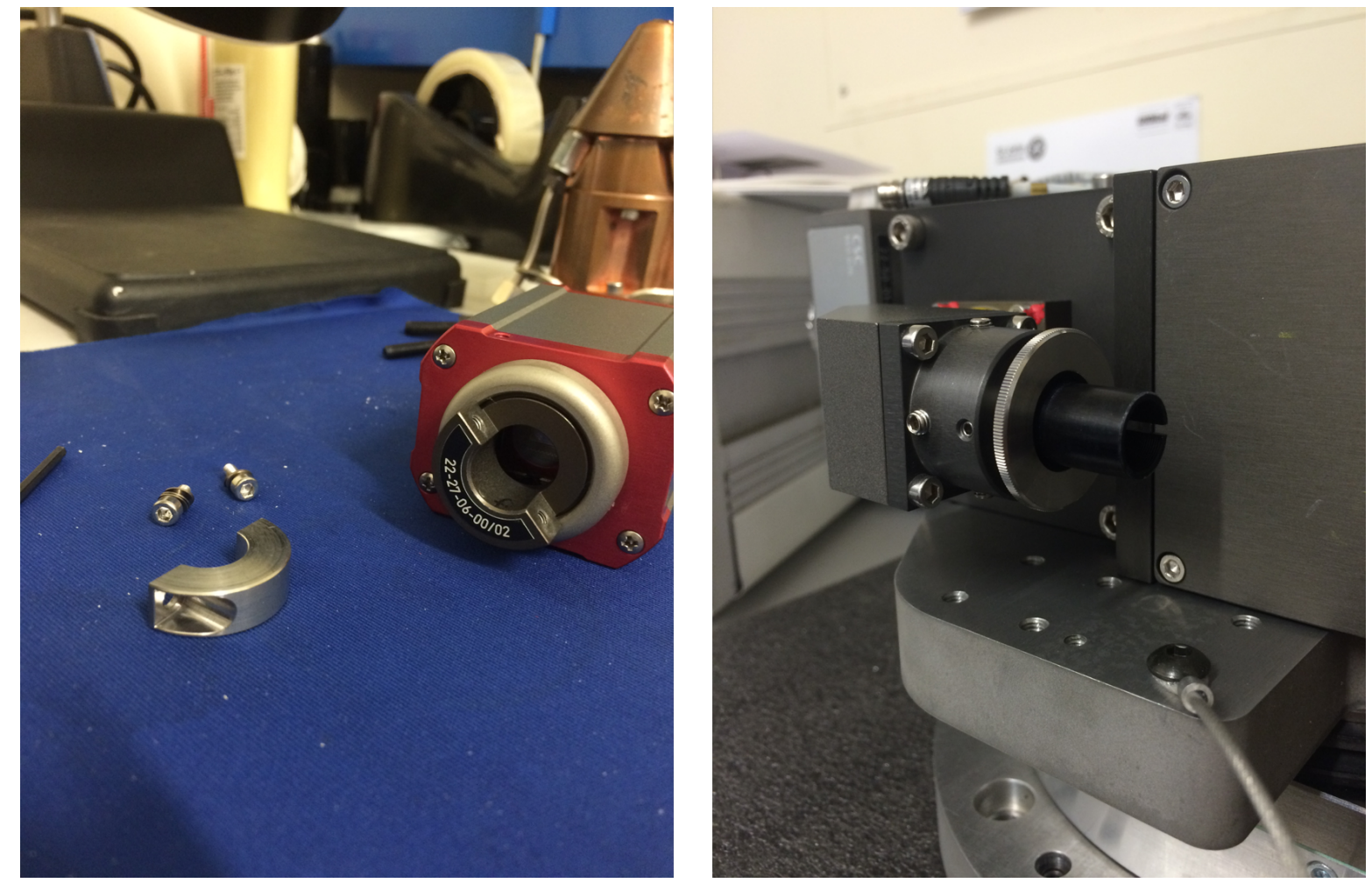

Figure 15: Left: CCD camera with C-mount mounting fixture. Right: Laser cladding head with exposed optical path. 


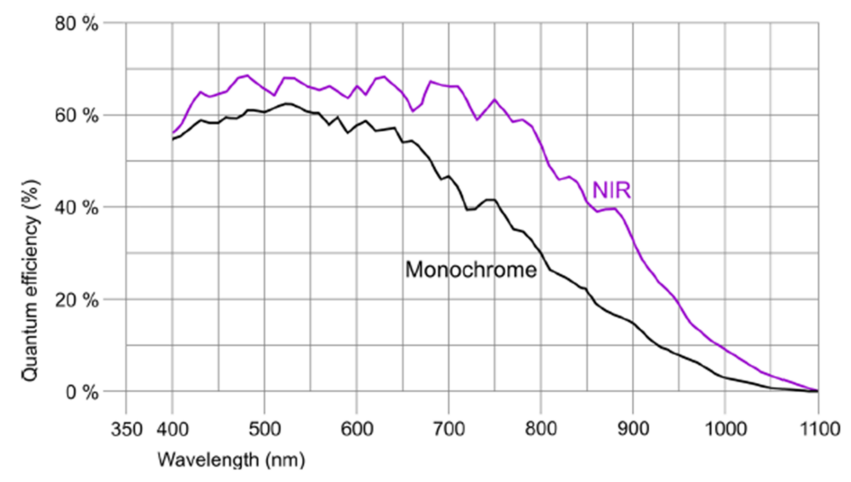

Figure 16: The quantum efficiency of the CMOS sensor used for melt pool imagery [74].

\subsubsection{Filtering Optics}

To aid the camera, a filter was used so that only a narrow band of wavelengths were permitted to strike the CMOS sensor. This reduced all visible light and eradicated IR radiation above 1000nm. A UV/VIS cut off imaging filter with a $135 \mathrm{~nm}$ notch was used. This filter has an optical density of 3.0 for wavelengths of $200-750 \mathrm{~nm}$ and an optical density of 4.0 for wavelengths of $1000-1200 \mathrm{~nm}$. The permitted radiation can be viewed in Figure 17.

The filter used in this set-up was crucial in reducing the total amount of saturation present in melt pool images. The notch filter reflects a large portion of electromagnetic radiation, reducing the total amount of incident radiation on the image sensor. This has the effect of reducing the amount of saturated pixels in melt pool images, which subsequently increases the amount of detail that can be observed at the specific permitted wavelengths. The upper limit spectral range for the filter was selected to increase the dynamical range of captured images. Imaging at wavelengths below $1,000 \mathrm{~nm}$ results in changes in temperature having more of an impact on measured signals [32]. The lower limits of this spectral range was selected to eradicate irrelevant visible light that could cause measurement deviations. Viewing the melt pool within this spectral range has the added benefits of being able to use silicon-based IR CMOS sensors. Advantages of using this technology has been discussed previously in Section 3.1.1.3. 


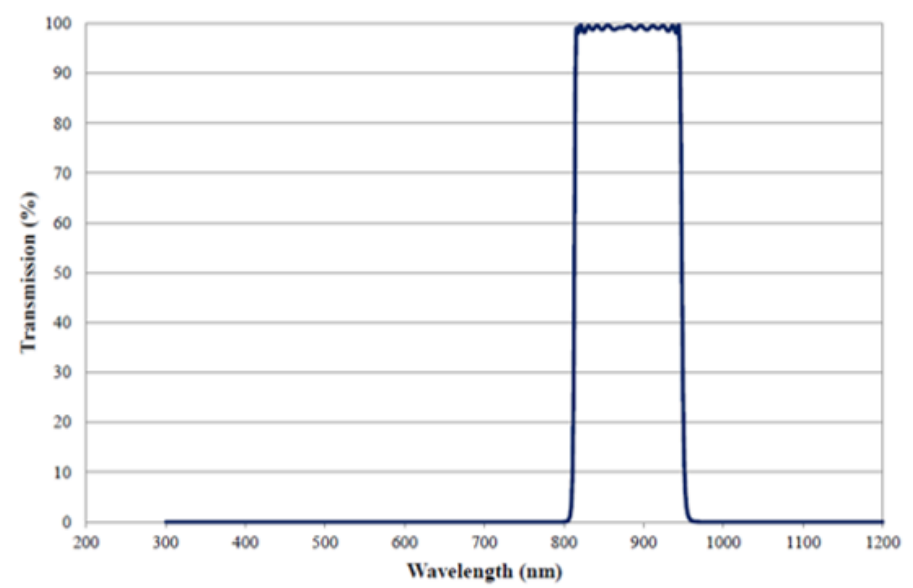

Figure 17: A graph showing the permitted wavelengths allowed through the UV/VIS cut-off filter [77].

\subsubsection{Optics Installation and Imaging Parameters}

The notch filter and NIR CMOS camera were coaxially installed into the Trumpf BEO D70 processing optics laser deposition head to allow for a birds-eye view of the melt pool. The old c-mount that was attached to the original CCD camera was removed, and a simple set of Allen keys allowed for the installation of the new NIR CMOS sensor. The newly installed camera is shown in Figure 18a. The imaging parameters are shown schematically in Figure 18b.

The new camera was connected to a laptop via a USB 3.0 connection and a series of tests were run to find the limitations of the system. Videos were recorded using the described optical set-up and software provided by IDS-Imaging. 

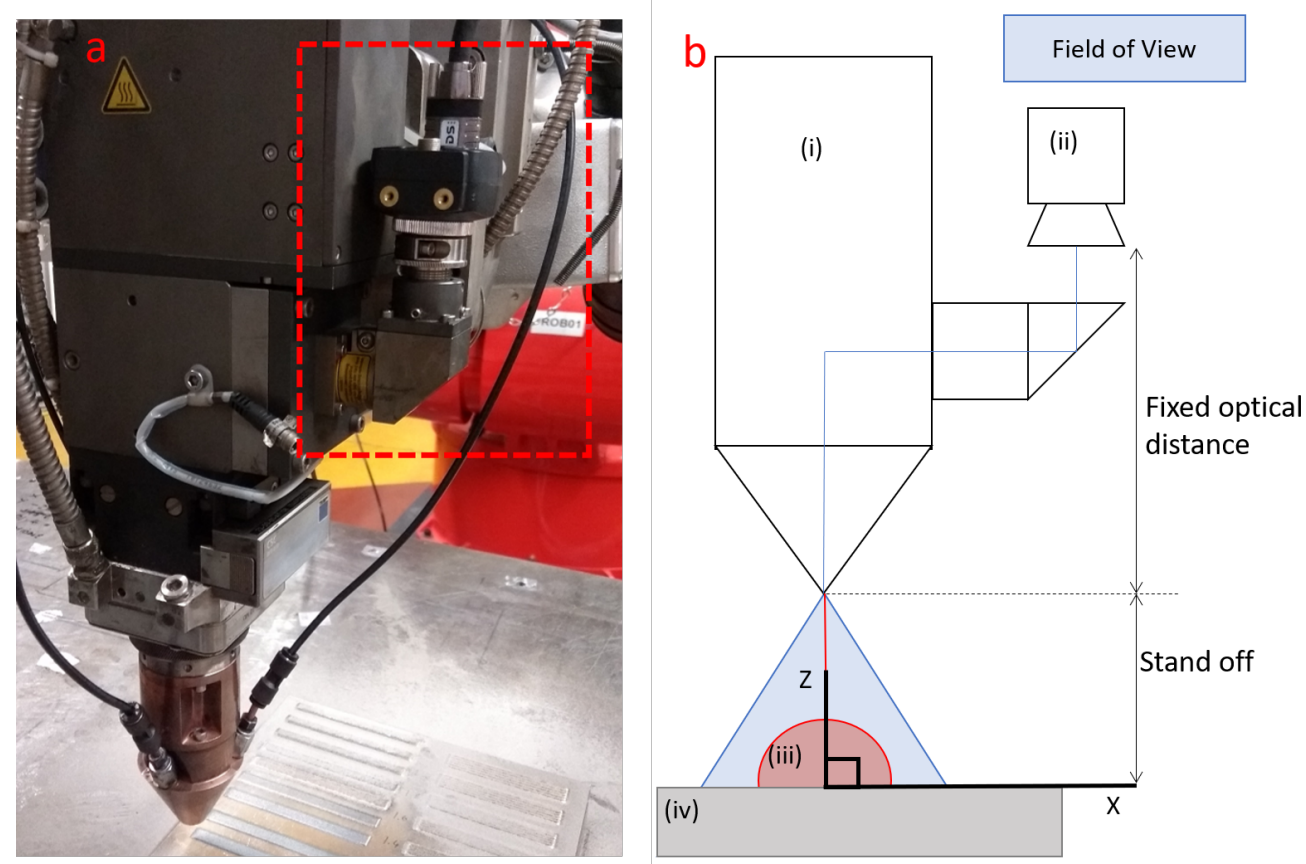

Figure 18: a) An image of the new camera installed on the laser deposition head. b) A schematic representing the stand off and FOV of the camera: (i) Laser deposition head. (ii) CMOS Camera. (iii) Melt Pool. (iv) Substrate

\subsubsection{Field Of View}

The FOV for the camera was restricted by the coaxial view through the nozzle. This was due to the internal optics of the Trumpf BEO D70 laser deposition head not being optimised for the NIR camera used in the experiments. Due to this, a portion of the CMOS sensor imaged the inside of the optical set-up and did not show melt pool information. The optics in the Trumpf BEO D70 processing optics module were fixed and could not be altered. Acquisition of technical information regarding the internal optics of Trumpf BEO D70 laser deposition head was attempted, but due to intellectual property issues this could not be obtained. Optical information of the Trumpf BEO D70 laser deposition head can be found here, but most of this information concerns laser processing optics rather than coaxial monitoring optics [83].

Due to the lack of internal optics information, and the total CMOS sensor capturing a portion of 
the inside of the deposition head, the FOV was calculated based on the total area of substrate that could be seen through the coaxial nozzle at a set stand off. To measure the FOV a ruler was placed on a surface bellow the viewing nozzle at a set stand off distance. A still image was taken, and a simple computer program was written to correlate pixel widths with millimetre measurements. At a stand off of $16 \mathrm{~mm}$, the horizontal and vertical FOV measurements were $6.6 \mathrm{~mm}$ and $6.6 \mathrm{~mm}$ respectively. A $16 \mathrm{~mm}$ stand off was used for all deposition throughout this entire research meaning the FOV did not change.

\subsubsection{Depth Of Field}

Due to the same issues regarding the lack of internal optics information, the Depth Of Field (DOF) could not be calculated in the conventional way (detailed in the following reference [84]). The DOF is the distance between the nearest and furthest objects giving a focused image. To calculate the DOF, the stand off distance was altered during deposition to determine at what point the system was deemed out of focus for the purpose of melt pool dimensioning. This provided an estimation of the systems limitations. The Depth Of Field (DOF) was found to be short for the optical set-up. The stand off was changed to determine the limitations of the system and it was discovered that the image became unfocused at a stand off of $18 \mathrm{~mm}$ and $14 \mathrm{~mm}( \pm 2 \mathrm{~mm})$. The DOF was approximately $4 \mathrm{~mm}$. Depositing at stand off distances outside these extremes produced poor quality melt pool images that lacked edge detail. This narrow DOF can result in poorly focused images if incorrect stand off distances are used, or heat accumulation causes the substrate to warp.

\subsubsection{Measuring Powder Mass Flow Rate}

Monitoring PMFR is an important part of optimising clad quality. The conventional units for measuring the PMFR in DED systems is $\mathrm{g} / \mathrm{min}$ and this section describes how the PMFR is calculated with the given set-up at TWI. Stating PMFR in units of $\mathrm{g} / \mathrm{min}$ allows for experimental repeatability.

The in-house system used to control parameters in this research does not include a method of being able to monitor the PMFR in detail at the time that it is being injected into the melt pool. Systems are available that can monitor the delivered PMFR in detail, but resources were not available 
to purchase a PMFR monitor for this research. Many studies measure the PMFR by the amount of weight delivered for a given time period. This section will outline how the PMFR is delivered, how the PMFR is set, and how this can be converted to grams per minute.

The PMFR parameter is set by selecting a percentage value on the in-house system. This percentage value corresponds to how much of the exit hole in the hopper is open. The higher the percentage, the more powder is allowed to pass through the system to the melt pool. To better quantify this value, a simple experiment was conducted to change percentage value into grams per minute. Many things can effect the total weight of powder that is delivered including the amount of carrier gas used, the density of the powder particles, and the random distribution of powder particle sizes.

To find out how much weight per minute was being distributed by the powder delivery system, a beaker was placed on a set of scales and set to zero before the powder delivery system was turned on. The powder delivery system was then turned on and powder was captured in a beaker on top of a scale. A minute timer was started. Once a minute timer finished the weight of the beaker was recorded. This was repeated for a series of PMFR settings to distinguish how the percentage gauge on the in-house system correlates to $\mathrm{g} / \mathrm{min}$ for the EN25 powder. Table 1 shows the conversion rates between system percentage and $\mathrm{g} / \mathrm{min}$ when using EN25 powder.

Table 1: The conversion rates between machine percentage and grams per minute for EN25.

\begin{tabular}{cc} 
Machine\% & Grams/min \\
\hline 9 & 3.7 \\
9.25 & 3.9 \\
9.5 & 4.0 \\
9.75 & 4.2 \\
10 & 4.3 \\
10.25 & 4.3 \\
10.5 & 4.5 \\
10.75 & 4.7 \\
11 & 4.8 \\
11.25 & 4.8 \\
11.5 & 4.9 \\
11.75 & 5.0 \\
12 & 5.2 \\
12.25 & 5.2 \\
12.5 & 5.4
\end{tabular}




\subsubsection{Powder Material}

EN25 powder was used for all deposition in this chapter. The chemical composition for EN25 is detailed in Table 2.

Table 2: The chemical composition for EN25

\begin{tabular}{ccccccccc}
$\mathrm{Al}$ & $\mathrm{Cr}$ & $\mathrm{Cu}$ & $\mathrm{Mn}$ & $\mathrm{Mo}$ & $\mathrm{Ni}$ & $\mathrm{P}$ & $\mathrm{Si}$ & $\mathrm{C}$ \\
\hline 0.003 & 0.69 & 0.006 & 0.64 & 0.59 & 2.66 & 0.004 & 0.26 & 0.32 \\
& & & & & & & & \\
$\mathrm{~S}$ & $\mathrm{O}$ & $\mathrm{N}$ & $\mathrm{Fe}$ & $\mathrm{TAO}$ & $\mathrm{B}$ & $\mathrm{Mg}$ & $\mathrm{Zr}$ & $\mathrm{Co}$ \\
\hline 0.003 & 0.02 & 0.003 & $\mathrm{Bal}$ & 0.01 & $<0.001$ & $<0.001$ & $<0.01$ & 0.01
\end{tabular}

\subsection{The Effects of Exposure Time on Melt Pool Imagery}

Obtaining an accurate representation of the molten metal pool in AM processes is a difficult task. Imagery of melt pools in referenced literature rarely shows the actual surfaces of a melt pool, and there has been little consideration to how both the substrate and the melt pool plume effect the digital image. Section 3.3 highlighted some key issues when comparing radiation mechanics to current melt pool images, especially when taking into consideration the directional emittance phenomena. To further explain how the directional emittance phenomena should be observed in correctly calibrated melt pool images, a schematic is represented in Figure 19. 


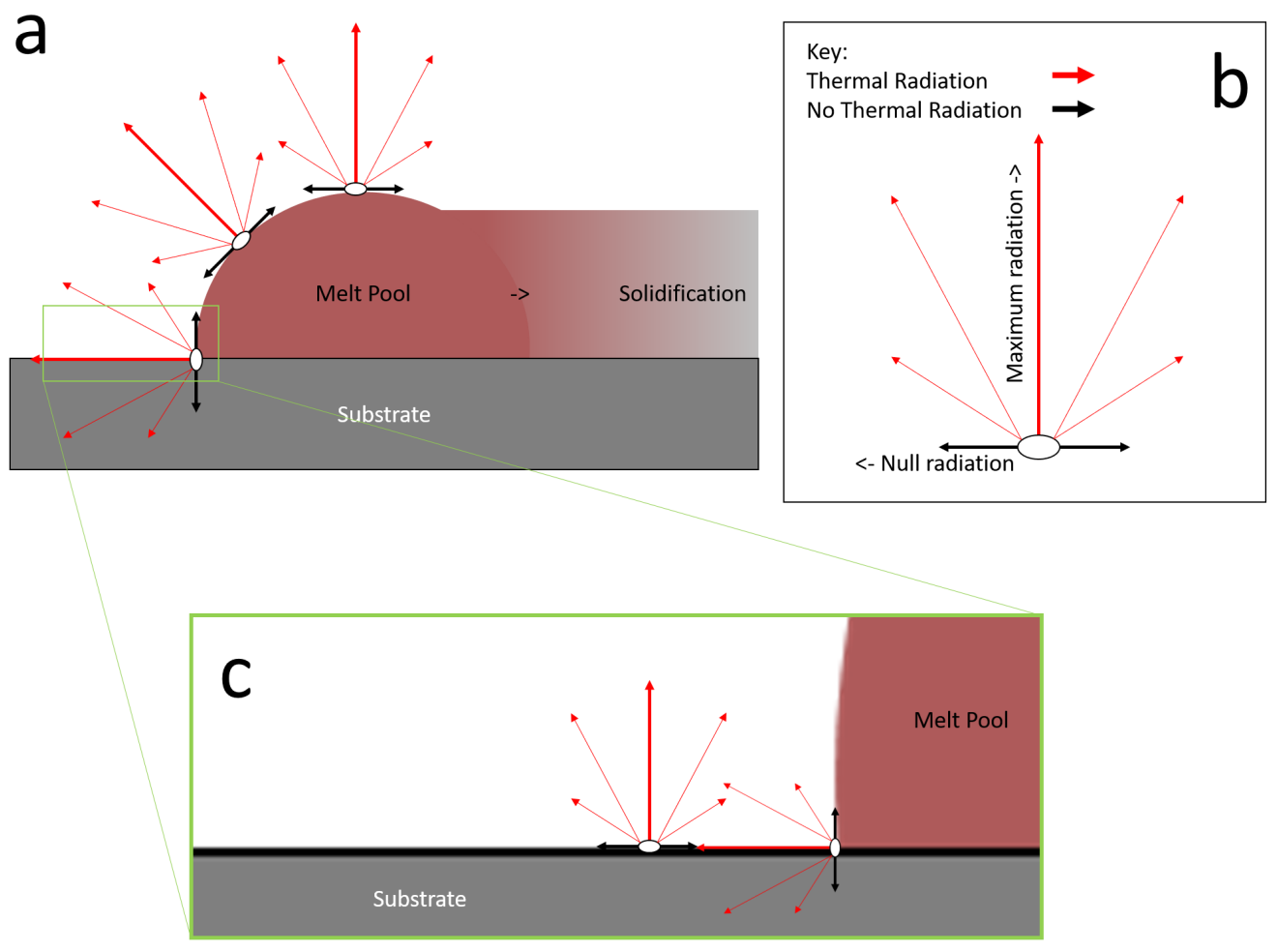

Figure 19: A schematic diagram representing how directional emittance effects digital imaging of melt pools. a) Melt pool, substrate and solidification track schematic. b) Directional emittance phenomena key. c) Close up schematic of the directional emittance phenomena at the melt pool edge.

Figure 19 superimposes the theory of directional emittance onto the melt pool scenario. The melt pool that is depicted in Figure 19 is not a blackbody and will therefore not emit radiation in equal magnitude in all directions. The radiation maxima will be normal to the surface, and radiation will not travel perpendicular to the surface that it is emitting from. Figure 19a shows how radiation from the top surface of the melt pool will emit maximum radiation in the direction of the camera. The melt pool edge shows null radiation upwards, meaning that the CMOS sensor should not receive EM radiation from this area. The surface of the melt pool will show maximum radiation intensity in the direction of travel but will in fact contribute no radiation directly towards the NIR CMOS sensor when a contact angle of $90^{\circ}$ is evident. 
Figure 19c shows an enlargement of the melt pool edge. As can be seen here, radiation from the melt pool will not be emitting in the direction of the CMOS camera, however radiation that is emitted from the substrate will have its maximum directed into the CMOS sensor. Theoretically, assuming a contact angle of $90^{\circ}$, the primary radiation from the substrate directly in front of the melt pool should contribute more towards the digital melt pool representation than radiation that is emitted from the melt pool edge. This phenomenon should be observed in cases where the contact angle is larger than $50^{\circ}$ and should be increasingly prominent as the contact angle increases towards $90^{\circ}$. Calculation of the contact angle in DED processes would be a difficult task and would have to consider not only the surface properties of heated metal materials, but also the forces that are exerted from powder particles, carrier gas, and shielding gas. This contact angle would change for different materials and most other parameters that are regularly changed in AM processes.

Taking into consideration all referenced work and a detailed understanding of both optical devices and radiation mechanics, the acquisition of a high quality melt pool image was attempted. For a qualitative analysis of melt pool images, a single laser power setting of $600 \mathrm{~W}$ was used for all cladding tracks. Four different exposure times were used $(0.5 \mathrm{~ms}, 1.0 \mathrm{~ms}, 1.5 \mathrm{~ms}$ and $2.0 \mathrm{~ms})$, and videos for the different tracks were compared. The direction of travel was fixed. The length of each track produced was $150 \mathrm{~mm}$. The carrier gas was set to $3.5 \mathrm{~L} / \mathrm{min}$. The shielding gas was set to $6 \mathrm{~L} / \mathrm{min}$. The path velocity was $11.25 \mathrm{~mm} / \mathrm{s}$. The laser spot size was $1.5 \mathrm{~mm}$. The PMFR was set to $4.8 \mathrm{~g} / \mathrm{min}$. The powder that was used in the processes was EN25. All settings remained constant throughout the experiment. The IDS video acquisition software was used to capture digital representations of all the cladding tracks for post build analysis. The effects of how exposure time and laser power both effect the digital representation of the melt pool and a qualitative analysis of what the user is seeing is discussed.

Figure 20 shows digital representations of the melt pool throughout 4 different cladding tracks. All the cladding tracks in this figure have the same fixed variables discussed in this section, they also have the exact laser power setting of $600 \mathrm{~W}$. The only variation between these images is the exposure time setting on the camera.

Figure 20a is taken with an exposure time of $0.5 \mathrm{~ms}$. This short exposure time does not allow for 

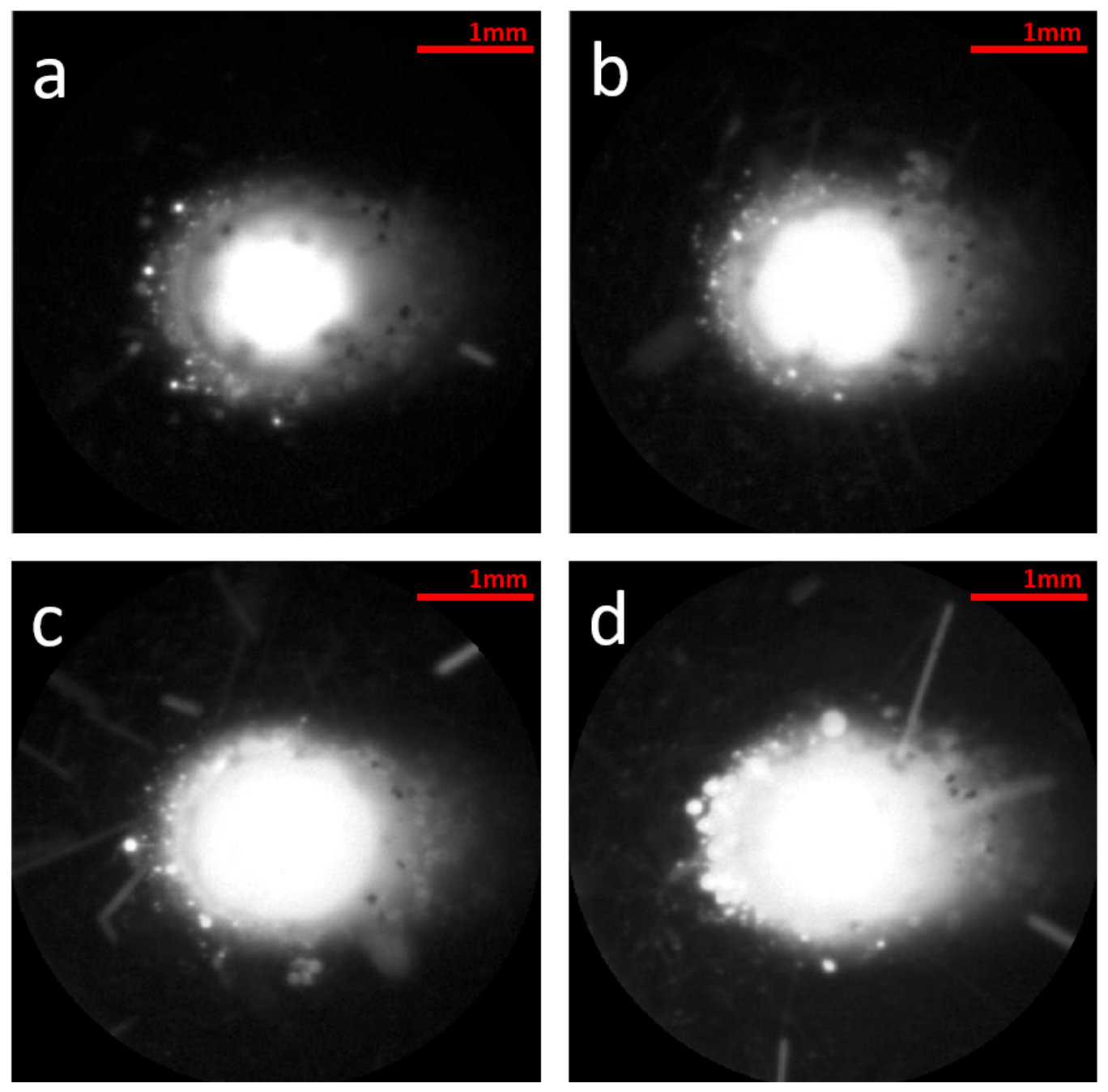

Figure 20: a - exposure time 0.5ms. b - exposure time 1.0ms. c - exposure time 1.5ms. d - exposure time 2.0.ms.

a large amount of radiation to enter the image sensor, resulting in a very low number of saturated pixels. The phenomena discussed in the previous section, regarding darkened regions around the melt pool (directional emittance phenomena), is evident in this image. A dark crescent is present at the front edges of the melt pool giving a clear indication of the melt pool width.

Figure 20b shows a melt pool that has the exact same input parameters but has an exposure time of 1.0ms. Increasing the exposure time allows for a better representation of the directional emittance 
phenomena. This image shows a clear dark region around the edge of the melt pool where the molten metal interacts with the solid substrate. By increasing the exposure time, radiation from the solid substrate surrounding the melt pool becomes more prominent, further highlighting the dark regions of the melt pool surface. The centre of the melt pool accumulates pixels that are fully saturated. This is because lower melt pool surface angles are allowed more time to contribute to the total radiation absorbed by the image sensor.

Figures 20c and 20d show the phenomena of directional emittance to be almost absent. The reason for this is due to a higher exposure time of $1.5 \mathrm{~ms}$ (bottom left) and $2.0 \mathrm{~ms}$ (bottom right). Increasing the exposure time allows for even more light to enter the image sensor, but this has a detrimental effect on melt pool image quality. The edge of the melt pool can no longer be seen due to high image saturation and radiation scattering in the atmosphere above the melt pool. This exposure time allows for the joint contribution of radiation emitted from both the melt pool and substrate. This increased exposure time also appears to make the melt pool look wider. Figure 20c shows a melt pool image that is very similar to those referenced in Figure 11.

If the exposure time of a camera is not properly selected for a certain laser power, then the true melt pool dimensions cannot be extracted. Selecting an exposure time too high will allow for melt pool radiation and substrate radiation to be indistinguishable from one another. Selecting an exposure time too low will make it difficult to distinguish the directional emittance phenomena. Selecting the correct exposure setting on the image sensor will allow for a clear melt pool edge feature. This feature can be used to extract accurate melt pool width measurements.

\subsection{Preliminary Exposure Time Experiment}

\subsubsection{Experimental Overview}

The previous section has identified the importance of exposure times and highlighted the need for optimised camera settings when calculating melt pool dimensions. Changing the exposure time can drastically change the image of the melt pool, and the directional emittance phenomena suggests that the darkened regions are a true representation of the melt pool edge. The previous section identified 
that having an exposure time too high can cause image saturation but having an exposure time too low can result in reduced contrast between the substrate and melt pool. The bright radiation that is emitted from the substrate surrounding the melt pool is a key tool in identifying the width of the melt pool. Selecting the correct exposure time is key when trying to obtain accurate melt pool dimensions.

In previous literature fixed exposure times have been used for all parameters settings. This experiment aimed to establish whether the same exposure time could be used for a range of different laser power settings. A series of different laser power settings are used to produce single line cladding tracks, and the exposure time on the camera was changed leading to the matrix of settings shown in Table 3. This section aimed to discover whether high quality melt pool images can be achieved using a single exposure time.

Table 3: Laser power and exposure time settings for preliminary exposure experiment

\begin{tabular}{cccccccc}
\hline ET & LP & ET & LP & ET & LP & ET & LP \\
\hline 0.5 & 600 & 1.0 & 600 & 1.5 & 600 & 2.0 & 600 \\
0.5 & 800 & 1.0 & 800 & 1.5 & 800 & 2.0 & 800 \\
0.5 & 1000 & 1.0 & 1000 & 1.5 & 1000 & 2.0 & 1000 \\
0.5 & 1200 & 1.0 & 1200 & 1.5 & 1200 & 2.0 & 1200 \\
\hline
\end{tabular}

ET - Exposure Time, LP - Laser Power.

\subsubsection{Method}

This experiment was carried out using the set-up discussed previously in Section 3.4, with the machine depositing single line tracks with lengths of $150 \mathrm{~mm}$. The direction of travel was fixed, the carrier gas was set to $3.5 \mathrm{~L} / \mathrm{min}$, the shielding gas was set to $6 \mathrm{~L} / \mathrm{min}$, the path velocity was $11.25 \mathrm{~mm} / \mathrm{s}$, the laser spot size was $1.5 \mathrm{~mm}$, and the PMFR was set to $4.8 \mathrm{~g} / \mathrm{min}$. The powder that was used in the processes was EN25. All settings remained constant throughout the experiment. Using the IDS-imaging software, videos were recorded for all different power and exposure time settings. After 
recording, videos were reviewed for a subjective analysis.

\subsubsection{Results}

Selecting an exposure time of $2.0 \mathrm{~ms}$ resulted in very little melt pool detail for nearly all laser power settings. The high exposure time caused image saturation for even the lowest laser power setting and resulted in no directional emittance features. Using a laser power setting of $1200 \mathrm{~W}$ in conjunction with this high exposure time resulted in high levels of image saturation and noise from powder particles being projected into the heated region. Figure 21 displays these issues.
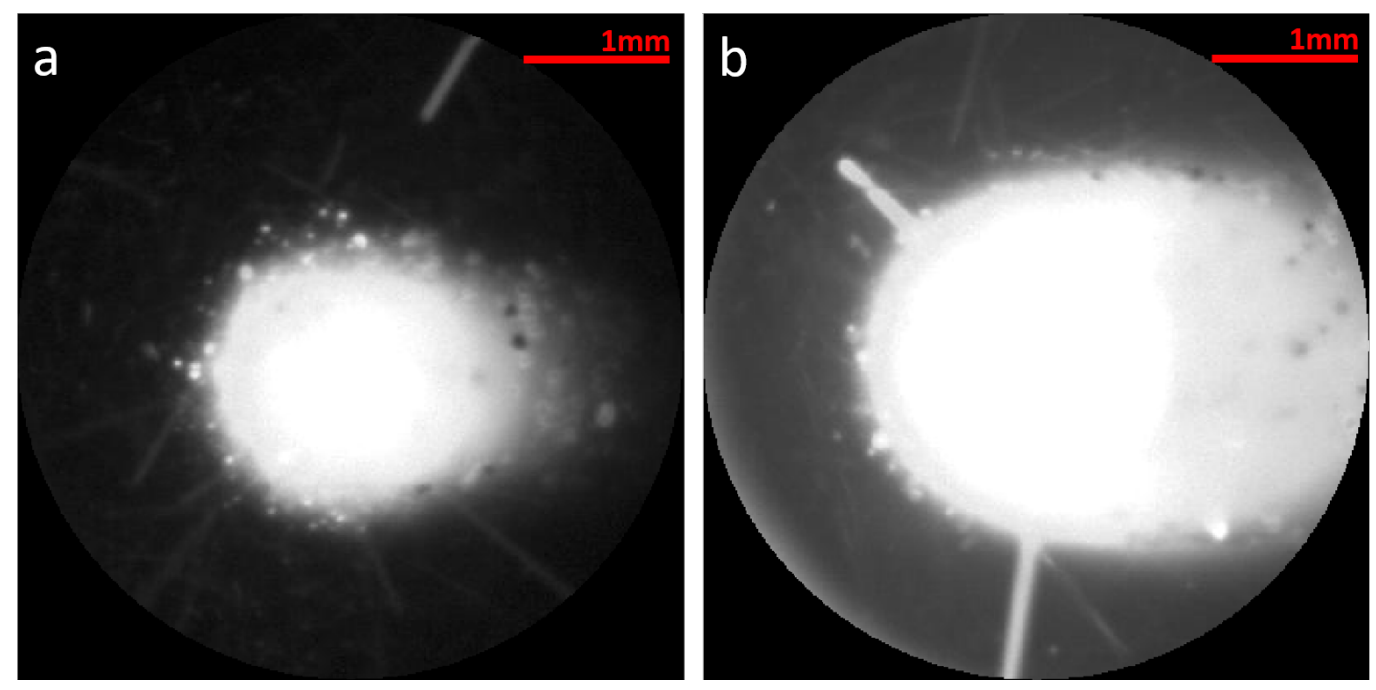

Figure 21: a: A typical melt pool image showing no directional emittance feature (exposure time $2.0 \mathrm{~ms}, \mathrm{LP}-600 \mathrm{~W})$. b: A typical melt pool image showing powder particles emitting large amounts of radiation (exposure time $-2.0 \mathrm{~ms}$, LP - 1200W).

Reducing the exposure time to $1.5 \mathrm{~ms}$ did not have a major impact on the melt pool images and many characteristics that were evident with the higher exposure time of $2.0 \mathrm{~ms}$ were evident again. The higher laser power settings of 1200, 1000, and 800W showed no directional emittance features. However, the dark region began to emerge on the lower power setting of $600 \mathrm{~W}$ in some frames (Figure 22).

The exposure time setting of $1.0 \mathrm{~ms}$ exposed more melt pool detail at all laser power settings. In 


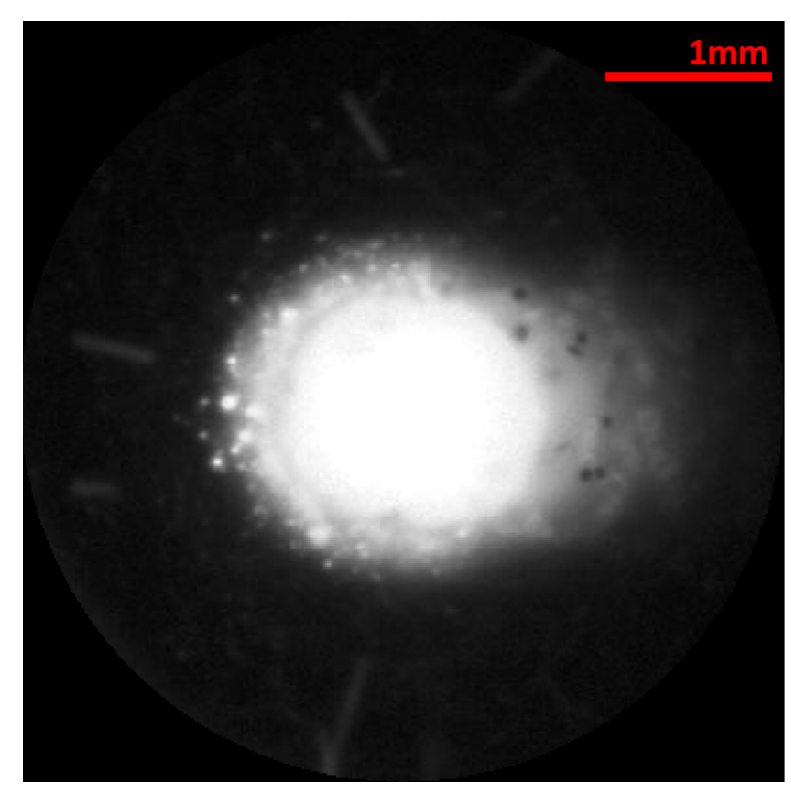

Figure 22: A melt pool still image displaying the melt pool dark region (exposure time - 1.5ms, laser power $600 \mathrm{~W})$.

nearly all frames on all laser power settings, the directional emittance feature surrounding the melt pool was evident. Figure 23 shows the directional emittance feature present in melt pool images at all laser powers.

Although the directional emittance feature can be viewed in all images, the feature is more clear in the lower laser power settings. Comparing the extreme laser power setting of $600 \mathrm{~W}$ and $1200 \mathrm{~W}$ it can be seen that the directional emittance feature is much smaller on higher laser power setting, and the image sensor detects less of the surrounding radiation from the solid substrate. This makes it harder for the user to view the melt pool edge and also for an image processing algorithm to detect. Not only is the melt pool edge clouded, but due to longer exposures there is a substantial amount of noise because of radiating powder particles. Figure 24 displays the issue of powder particles misrepresenting the melt pool.

At an exposure time of $0.5 \mathrm{~ms}$, all videos showed the directional emittance feature. Figure 25 compares still images taken from the 600W deposition tracks. Figure 25a is taken with a lower exposure time of $0.5 \mathrm{~ms}$, and Figure $25 \mathrm{~b}$ is taken with an exposure time of $1.0 \mathrm{~ms}$. The directional 

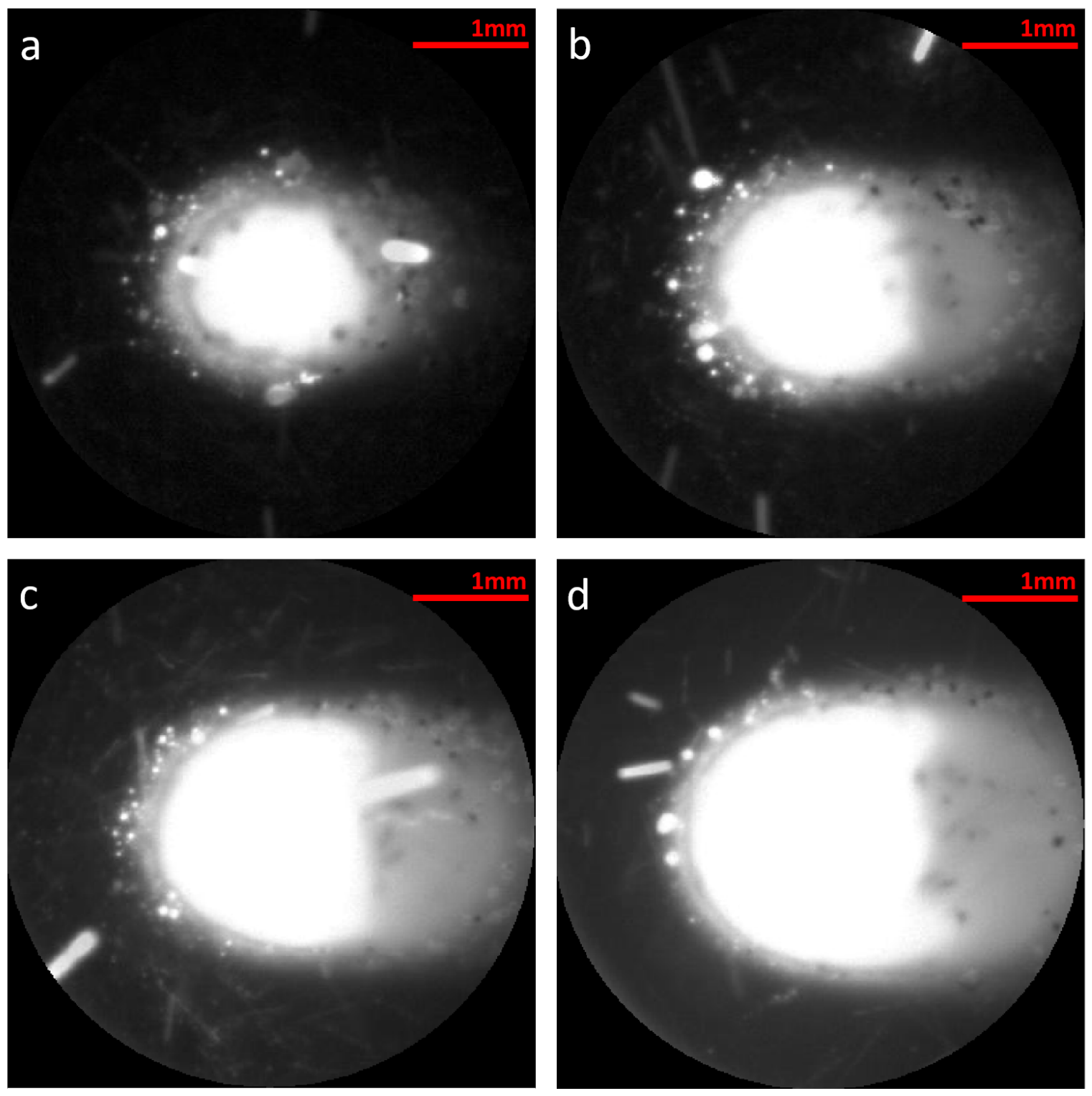

Figure 23: Melt pool images displaying the directional emittance feature. The exposure times for all images are $1.0 \mathrm{~ms}$. The laser powers are: $\mathrm{a}-600 \mathrm{~W}, \mathrm{~b}-800 \mathrm{~W}, \mathrm{c}-1000 \mathrm{~W}, \mathrm{~d}-1200 \mathrm{~W}$.

emittance feature on the $1.0 \mathrm{~ms}$ image is more prominent than the one displayed on the $0.5 \mathrm{~ms}$ image due to substrate surface radiation providing a contrast to the dark region at the edge of the melt pool. Reducing the exposure time too much can reduce the amount of radiation that is detected from the 

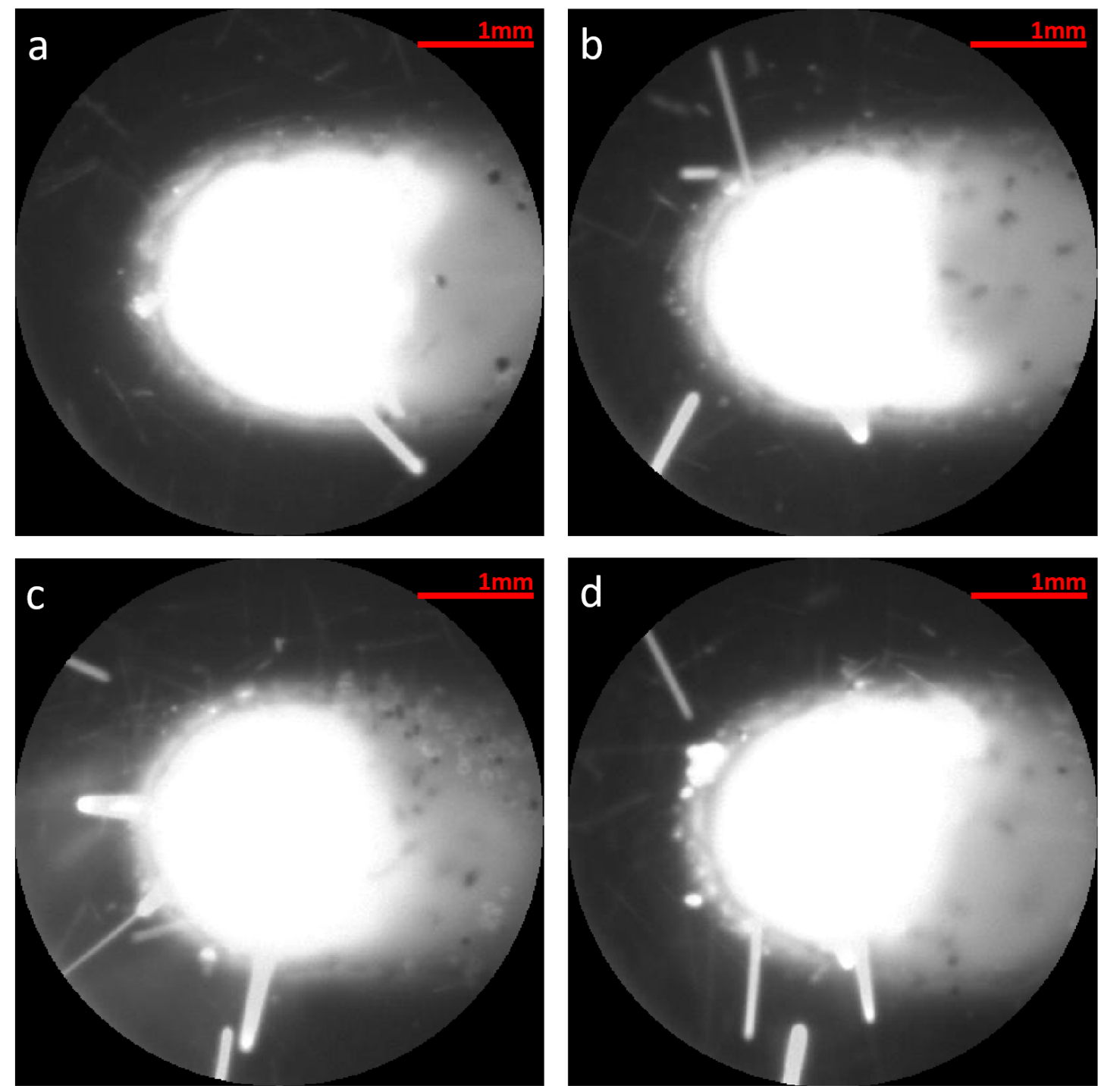

Figure 24: Four still images taken from a recording of a single line deposition (exposure time - 1.0ms, laser power - $1200 \mathrm{~W})$.

emitting substrate, causing the once observed directional emittance feature to blur into the underlying substrate. Although the edge feature can be partially seen in some of the frames, it is not clearly indicated throughout the entire video with the $0.5 \mathrm{~ms}$ exposure time. For this reason, it would be difficult to identify the dimensions of the melt pool using image processing algorithms. Analysing the 
two different exposure settings on the $800 \mathrm{~W}$ power setting provided a similar conclusion.
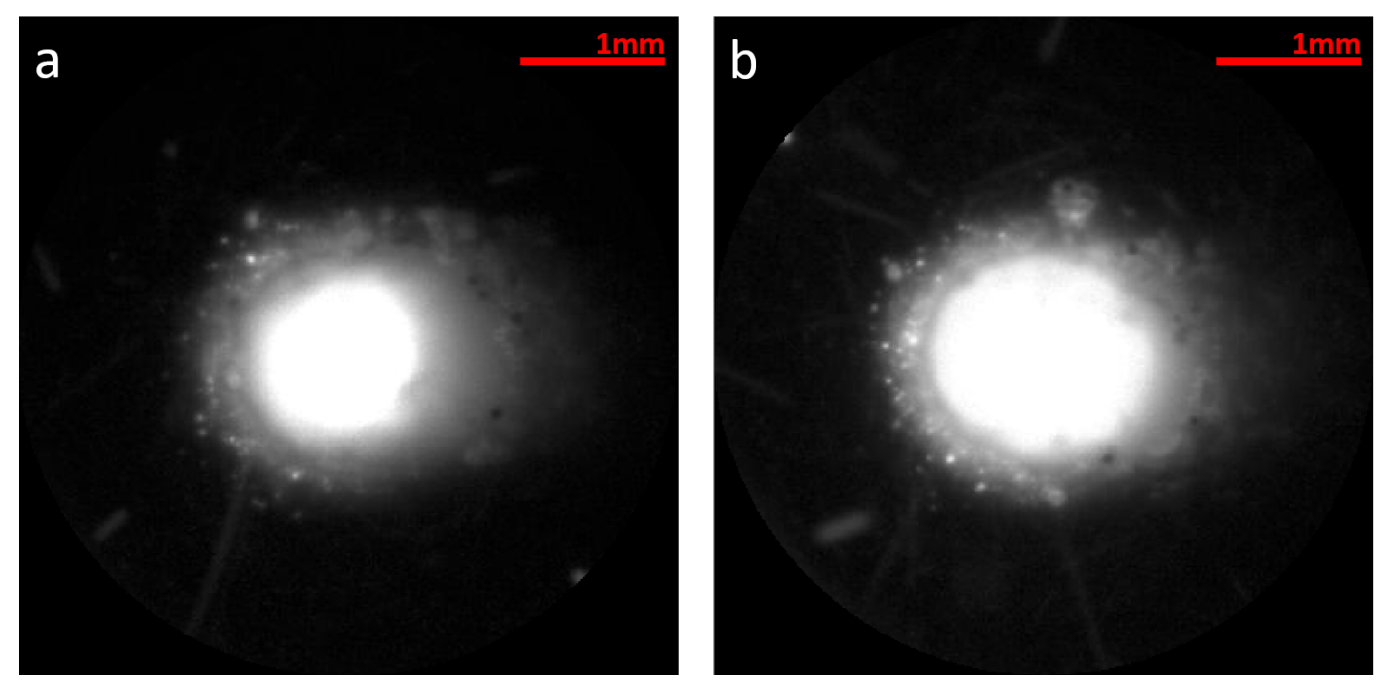

Figure 25: a - A still image from a recording of track deposition (exposure time - 0.5ms, laser power $600 \mathrm{~W}$ ). b - A still image from a recording of track deposition (exposure time - 1.0ms, laser power $600 \mathrm{~W})$.

The higher laser power settings of $1000 \mathrm{~W}$ and $1200 \mathrm{~W}$ showed more detail of the melt pool with the lower exposure time. By reducing the exposure time to $0.5 \mathrm{~ms}$, the number of noisy frames, due to stray powder particles (as seen in Figure 24) was reduced. By keeping a low exposure time, stray particles that were causing unwanted flares are reduced in two ways. Keeping the low exposure time first means that powder particles emitting radiation are less likely to cause high intensity pixel values as less radiation is being received. More importantly though, the size of the powder particles viewed are greatly reduced. The flares that are evident in Figure 24 cover more pixels due to longer exposures. By having a longer exposure time, the particle that is emitting radiation can travel further across the CMOS sensors FOV, causing long flares of saturated pixels in the final image. Figure 26 shows a still melt pool image, at a laser power setting of $1200 \mathrm{~W}$, with an exposure time of $0.5 \mathrm{~ms}$. This figure shows a typical still frame which is subject to flares. The size of the flares are greatly reduced due to lower exposure times. 


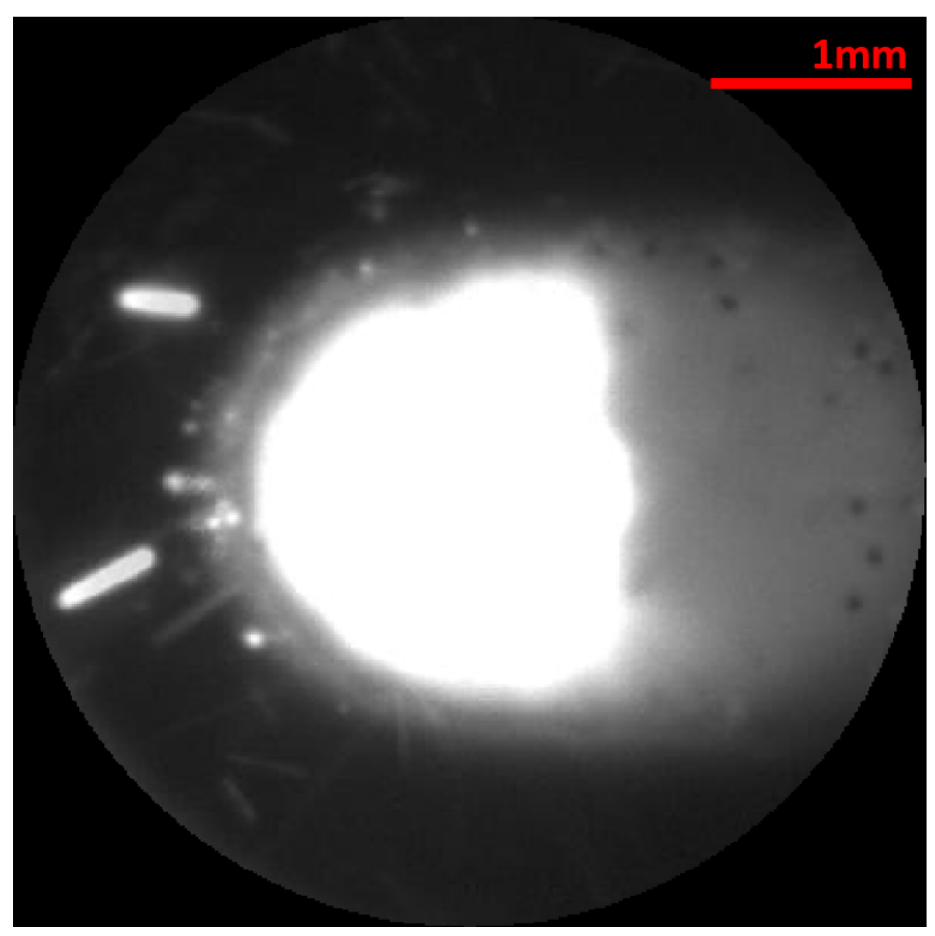

Figure 26: A still image taken from a video recording of track deposition showing a reduced number and magnitude of flares (exposure time - 0.5ms, laser power - 1200W).

\subsubsection{Conclusion}

It has already been established that the correct exposure time should adequately highlight the directional emittance feature. This feature is a clear indication of the melt pools' edge and can be used in conjunction with image processing techniques to give an accurate measurement of the melt pool width. To highlight this feature a correct exposure time must be selected. Not only must this feature be evident, but it should also be prominent to allow for easy detection by new image processing techniques. The more prominent the contrast between the melt pool edge and the substrate, the more accurate and reliable the image processing calculations are likely to be.

This section of work has highlighted the need for an adaptive exposure time that is dependent on the laser power settings used in DED processes. To achieve a high quality melt pool image, a specific exposure time must be selected. This exposure time is dependent on the laser power setting. Exposure 
times too low result in no distinct melt pool features that can be extracted using image processing techniques. Exposure times too high result in undesirable flares and no distinct melt pool features that can be extracted using image processing techniques. Understanding this, the following section has been designed to allow for quality melt pool images to be obtained at all laser power settings.

\subsection{Adaptive Exposure Times for Varying Laser Power Settings}

\subsubsection{Experiment Overview}

There is a clear need to change the exposure time for different laser power setting during AM processes. The aim of this experiment is the development of a machine vision technique that can produce clear melt pool images for a range of different laser power settings. Melt pool monitoring systems need to be able to accurately measure geometric features for a variety of different system settings and materials. A system that is unable to measure these features will not give an accurate representation of the melt pool, and thus cannot determine how the melt pool is behaving in the complex DED process.

\subsubsection{Method}

The set-up used for this experiment is the same as that previously described in Section 3.4. The installed coaxial camera was used to view the melt pool throughout the DED process. A series of different laser power settings were selected, and the exposure time was changed to correct for increases/decreases in the thermal radiation. Single line tracks with lengths of $150 \mathrm{~mm}$ were deposited onto a $15 \mathrm{~mm}$ thick $316 \mathrm{~L}$ stainless steel substrate. The powder that was used in the processes was EN25. Twenty-one different laser power settings were used for deposition starting at $600 \mathrm{~W}$ and ending at $1200 \mathrm{~W}$ with $30 \mathrm{~W}$ increments. For each given laser power setting, three relevant exposure times were selected to determine the interaction between these two variables.

The method of calculating the exposure time for this experiment was arbitrary. It was undertaken by using a subjective decision made by a single operator. The single operator selected three different exposure settings for a given laser power setting to indicate a 'best' exposure time setting, as well as a 'high' and 'low' threshold limit. The exposure time increment for the experiment was $0.025 \mathrm{~ms}$. 


\subsubsection{Exposure Time Criteria}

The best exposure setting was determined when the operator deemed two image features to be at their most prominent. The first feature that the operator looked for was low image saturation in the centre of the melt pool. The second feature that the operator looked for was a prominent directional emittance feature indicating the edge of the melt pool. The exposure time was incremented until the directional emittance feature was most prominent and there was low melt pool image saturation. The best exposure time setting should produce an image that can easily resolve the melt pool edge, whilst simultaneously providing additional information about the molten metal.

The low exposure setting was determined when the operator observed merging between the melt pool and the surrounding substrate. This occurs when the exposure setting is too low, and not enough light is allowed onto the CMOS sensor. The lower bound exposure setting was selected when the given exposure time could resolve the darkened melt pool region for a majority of frames, but an exposure time at one increment below could not.

The high exposure setting was determined when the operator observed merging between the melt pool and the surrounding substrate. This occurs when the exposure setting is too high, causing high image saturation. As the image saturation begins to increase, the melt pool centre and surrounding substrate will be represented by more pixels with maximum values. Continuing to increase the exposure time will cause pixels representing the melt pool centre and heat affected substrate to merge together. This removes the directional emittance feature, indicating the melt pool edge, from the image. The high exposure setting was selected when the given exposure time could resolve the directional emittance feature for a majority of frames, but an exposure time at one increment above could not.

\subsubsection{Exposure Time Acquisition}

To collect the three exposure times for a given laser power setting, single line tracks were deposited onto a fresh substrate in a continuous loop. Repeatedly depositing tracks in quick succession allowed for an iteration of the exposure time setting, whilst simultaneously observing a live image feed of the melt pool deposition track. The observation technique highlighted above was used to give a quantitative 
exposure time value based on the operator's view of the live image feed. The first $150 \mathrm{~mm}$ track was used to extract rough preliminary values for the low, high and best exposure times. Further deposition tracks were then used to verify these values, and subsequently allowed for a more detailed analysis and finer exposure time tuning. At least three $150 \mathrm{~mm}$ deposition tracks were used to validate the exposure time setting for a given laser power once the operator had decided on a value. Repeat measurements were carried out if the validation tracks did not support the observer's initial decision. For each laser power setting a high, low and best exposure time was recorded.

\subsubsection{Experimental Results}

Once all the exposure times were selected and verified in live mode, tracks were deposited and recorded for qualitative analysis. A total of fifteen videos were recorded using the low, best and high exposure times settings for deposited tracks with fixed laser power settings of 600, 750, 900, 1050, and 1200W. The tracks deposited were $150 \mathrm{~mm}$ in length. The still images depicted in Figures 28 and 29 were sourced from these videos.

Figure 27 shows a linear relationship between exposure time and laser power when following the operator's criteria outlined. For the low, best, and high scenarios, decreasing the exposure time allowed for homogeneity in live melt pool image feeds for increasing laser powers. Increasing the laser power causes more thermal radiation to be emitted from both the melt pool and surrounding substrate. This excess radiation can cause image saturation, which is known to cause false readings when image processing algorithms attempt to extrapolate melt pool dimensions. To counter this phenomena, the camera's exposure time is reduced to limit the total amount of radiation that the CMOS sensor is exposed to. The results from the described experiment show that the relationship between exposure time and laser power is linear for the given material deposited at all laser powers between $600 \mathrm{~W}$ and $1200 \mathrm{~W}$. This linear approximation can be used to observe the direction emittance phenomena at a range of laser power settings successfully. Because of this, data from the experiment has been used to create Equation 1. This equation can inform the user what exposure setting should be selected dependent on the laser power setting used whilst depositing with the material EN25. This equation is a line of best fit and is only repeatable for this specific EN25 powder. This is likely to change for both 


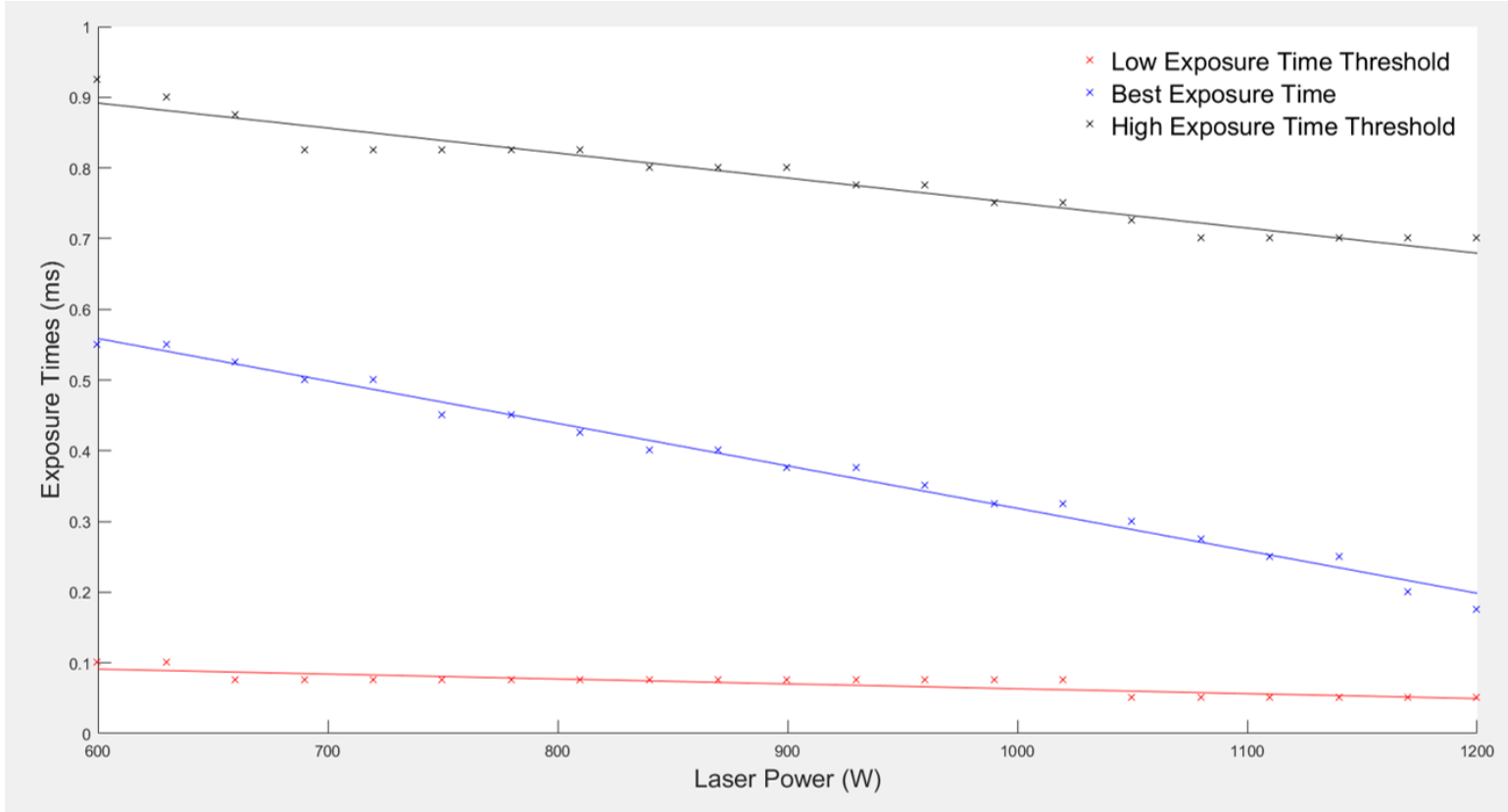

Figure 27: A graph displaying the relationship between the low, best, and high exposure times and laser power settings in DED processes.

different materials and different powder manufacturers.

$$
E=-6.0 \times 10^{-4} \cdot P+0.9192
$$

Where $E$ is the exposure time setting to be used, and $P$ is the laser power setting being used when depositing with the material EN25.

The red line, that indicates the low exposure time threshold, has a much shallower gradient than the other two lines. The incremental step of $0.025 \mathrm{~ms}$ for exposure time resulted in large jumps in melt pool visibility at low exposure time settings. The convergence of the red and blue lines, as the laser power increases, indicates that using a step of $0.025 \mathrm{~ms}$ will likely be inadequate for deposition with higher laser power settings. When operating at higher laser powers (1200W and above) finer tuning of the exposure time may be required. Further analysis of this relationship could provide more information as to exactly why these two lines converge and perhaps expose a different relationship to the assumed 
linear approximation. The causation of different gradients could be down to the observation criteria previously outlined. The three exposure time settings for a given laser power were chosen based on the operator's view of different key features observed in live image feeds. Different criteria for low, best and high exposure time settings are likely to cause a variance in line gradient.

Figure 28 displays images taken from the recorded videos of tracks deposited with laser power settings of $600,750,900,1050$ and $1200 \mathrm{~W}$. These evenly spaced laser power distributions show images with the same key characteristics and similar intensities. None of the images show high melt pool saturation in the centre of molten material. None show high levels of merging between pixels representing the centre of the melt pool and the heat affected substrate. All images show the directional emittance feature. Added features that have not been witnessed in previous images such as dark regions within the melt pools themselves can also be seen. The images, especially those with higher laser power settings, display a melt pool with a trailing tail. Quick analysis of these five images indicates that this tail becomes more prominent with increased laser power. The angle of the tail can also be seen.

Figure 29 displays images taken from low and high exposure time videos for laser powers of 600 , 750, 900, 1050 and 1200W. The low exposure time settings display the last exposure time increment before the directional emittance feature is no longer visible. All images look similar to each another, the directional emittance feature is barely visible, and an image processing algorithm would find it difficult to accurately measure melt pool dimensions on these frames. The high exposure time settings display the last exposure time increment. One increment above this value will see frames having pixels from the melt pool and surrounding substrate merging with each other. These frames lack the directional emittance feature, which is caused by high image saturation. An image processing algorithm would find it difficult to accurately measure melt pool dimensions on these frames. 

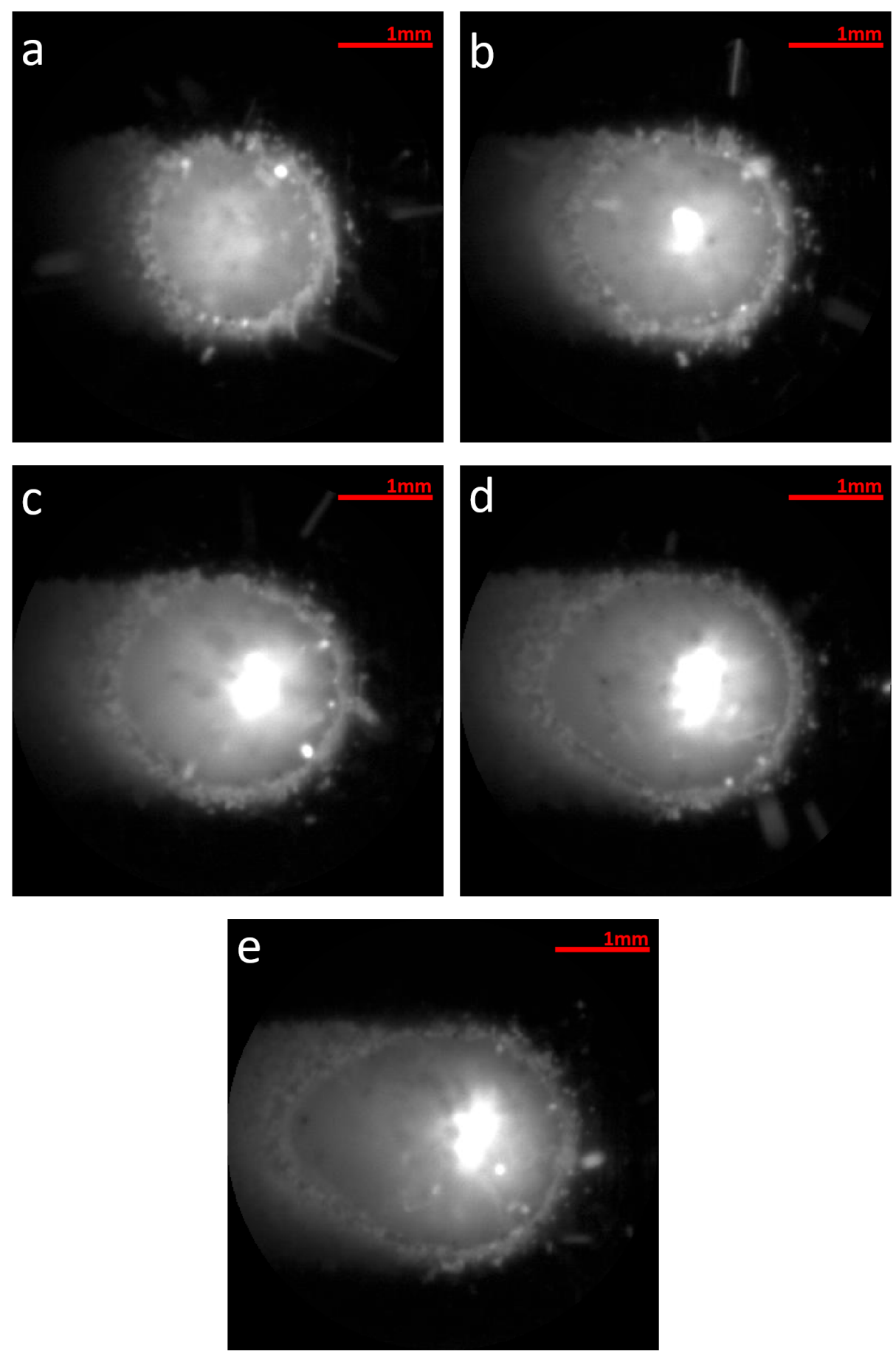

Figure 28: A series of melt pool images for various laser powers taken using the best exposure time. The laser power settings are: a.600W b.750W c. $900 \mathrm{~W}$ d. $1050 \mathrm{~W}$ e. $1200 \mathrm{~W}$ 

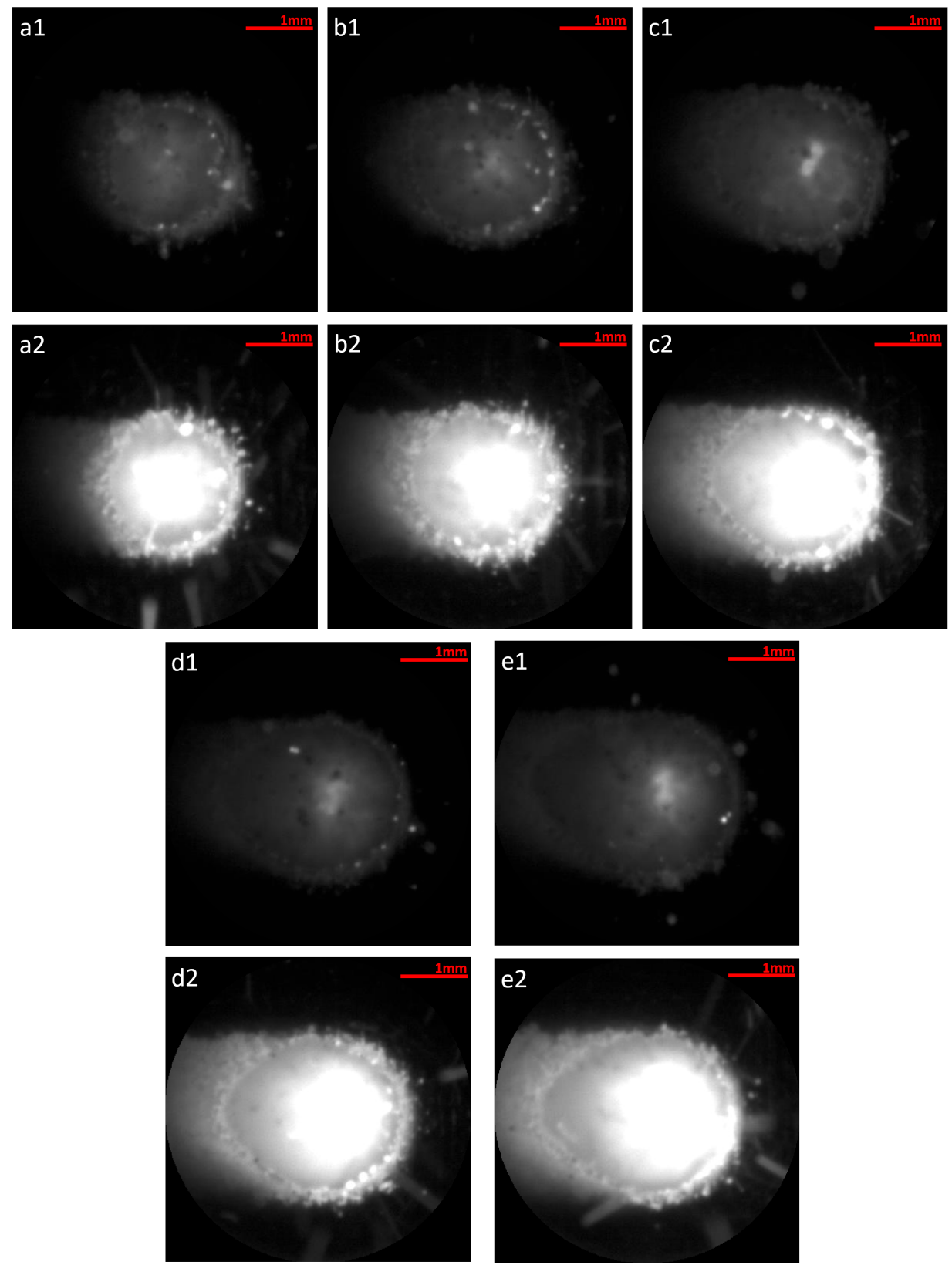

Figure 29: A series of melt pool images for various laser powers taken using the low $(* 1)$ and high $\left({ }^{*} 2\right)$ threshold exposure times. The laser power settings are: a.600W b.750W c. $900 \mathrm{~W}$ d. $1050 \mathrm{~W}$ e.1200W 


\subsubsection{Conclusion}

This experiment has resulted in high quality melt pool images and has demonstrated that improved images can be achieved by reducing the exposure time setting on a CMOS camera for increased laser powers. The relationship between laser power and exposure time for the specific material and laser powers used appears to be linear. For subsequent algorithm development, this linear approximation will be assumed.

The designed experiment uses no image processing algorithms, because at this moment in time no suitable image processing algorithms have been created. The Canny edge image processing algorithm, which will be discussed in detail in the following chapter, cannot determine the size of the melt pool accurately. The algorithm does not take into consideration that the heat affected surface emits radiation at a higher intensity than parts of the molten metal surface in the direction of the coaxial CMOS camera. Rather than clouding the phenomena of directional emittance, this experiment sought to highlight it for subsequent algorithm development. This has not only been successful, but it has also highlighted features within the melt pool that have not been documented previously in DED systems.

This technique has produced high quality images at a range of different laser power settings, but has introduced an element of subjective human decision making. This technique requires an individual to select the best exposure time setting based on his/her opinion, resulting in likely variance between different users and different measurements.

An alternative to this would be to introduce a more autonomous method that adapts exposure time based on image processing techniques. One idea would be to reduce/increase the exposure time setting on the camera until a specified number of pixels are saturated (for example an area between 10 - 1000 pixels in an image). A more complex analysis could use an image processing technique to measure the directional emittance phenomena and change the exposure time to maximise its prominence. Both these techniques would eradicate the need for a subjective decision and would likely increase the systems robustness and image quality.

Selecting a highly accurate quantitative exposure time value for a given laser power using the operator's criteria was difficult, but most importantly not necessary. The new algorithm that is to be 
developed will aim to distinguish the true outline of the melt pool by using the directional emittance phenomena (dark region). The position of the dark region does not change with varying exposure times. Changing the exposure time of the camera when viewing the melt pool in this experiment only changed the prominence of the directional emittance feature, not its location. This experiment does not provide an explicit exposure time that has to be used for a given laser power, but rather a window of exposure times that can be used for a range of laser power settings. The best exposure time is a recommendation to allow for more reliable image processing algorithm results. An ideal algorithm that works based on the directional emittance phenomena should be able to operate within the area underneath the black line and above the red line in Figure 27. The algorithm designed should provide the same dimensions for the melt pool, at a given laser power, for any exposure time between the low and high thresholds. The best value should be used as a guide to allow for optimum melt pool dimensioning and will reduce the amount of incorrect measurements that the algorithm may produce. A system that incorporates this technique will allow for real time melt pool dimensioning independent of both the camera's exposure time, and a fixed threshold value used in the Canny edge image processing method. Future work aims to develop a diverse parameter monitoring system that can work at a range of laser powers and different materials without the need for lengthy calibration procedures.

\subsection{Discussion}

A majority of the melt pool images that have been referenced, and used to dimension the melt pool, show high levels of saturation $[27,34,45,46,69]$. This high level of saturation in melt pool images greatly reduces melt pool detail, and results in a lack of definitive melt pool edge features. This chapter aimed to increase the level of detail, reduce the amount of saturation, and highlight edge features in melt pool images. Melt pool monitoring and melt pool width dimensioning has proved to be an incredibly useful tool in advancing AM capabilities [10, 17, 23-34, 43-58], so it is of high importance that these techniques are accurate and provide high quality melt pool images.

This research used notch filters and low exposure times settings to achieve high quality melt pool images. This research also explored the use of adaptive exposures times to achieve high quality melt 
pool images at a range of different laser power settings. Combining low exposure times, notch filters and adaptive exposure time techniques resulted in high quality melt pool images with low levels of saturation for a range of different laser power settings. These high quality melt pool images show enhanced detail when compared with other start of the art melt pool imagery [27, 34, 45, 46, 69]. Other melt pool monitoring systems claim to be able to measure melt pool width dimensions [10, 23$25,27-31,33,34,39,40,43-47,67]$ but these use constant emissivity values that have been documented to be unreliable [39, 40, 47].

Achieving high quality melt pool images at a range of parameter settings is important to allow for accurate process monitoring. There are many factors within DED processes that are known to effect both the radiation and size of the melt pool. Some of these variables are controllable and other are not. Nozzle blockages and uneven powder distribution can have a major influence on melt pool geometry and can occur randomly throughout the AM processes [23]. Geometric variances including starting and finishing cladding tracks, direction changes, corners, multiple layer builds, overlapping and overhangs also have a major influence on clad properties and are known to effect build quality $[10,15,19,28,30,31,35,36,48,52,57,59,65]$. Observing the effects of these parameters on melt pool geometry can provide a better subjective understanding of the AM process and allow users to predict defects and optimise clad quality [10, 15-17, 19, 28-31, 33, 35, 36, 39, 40, 43-46].

Although results show high quality melt pool images at a range of different laser power settings, the equation created (Equation 1) is only viable for the specific batch of EN25 powder used. Changes in chemical composition and powder particle size would likely require recalibration of the exposure times for different laser power settings. This said, Equation 1 shows a straight line approximation, meaning that recalibration could be easily performed for new materials and powders. The feasibility of optimising exposure times for extreme laser power settings (min and max) and extrapolating between these values to calculate all exposure times settings should be explored.

Limitations of this work are that the calculation of both FOV and DOF could not be conventionally performed due to the unknown internal optics of the Trumpf BEO D70 laser deposition head. Though exact calculations of both the FOV and DOF could not be achieved using conventional techniques (detailed in the following references $[84,85]$ ), good estimations of both FOV and DOF were acquired. 
Future developments of the designed melt pool monitoring system should look to advance the optical set-up used. Optimising the internal optics could allow for more of the CMOS sensor to be utilised in viewing the melt pool. This in turn would lead to better melt pool resolution and detail. An improvement of optics should also look to increase the DOF the system. Increasing the DOF will allow for users to use this system at a larger range of stand off distances, and increase the systems robustness in the event of overbuilds, underbuilds and substrate warping.

The optimisation of system optics will likely require a substantial amount of dedicated research and collaboration with laser deposition head manufactures. Although this is an important aspect of the melt pool monitoring system, this research focuses on the use of optimising exposure times to highlight melt pool details and using these images to design advanced melt pool dimensioning algorithms. Optimising the optical set-up should be performed after the feasibility of these techniques are explored to improve the systems robustness and image quality.

Future work should look to utilise melt pool imaging advancements performed in this chapter by analysing the accuracy of conventional melt pool dimensioning techniques. This analysis can be done by comparing measurements to the newly discovered edge features. In conjunction with this testing, new image processing algorithms should be developed that can more accurately dimension the melt pool by utilising the new edge features.

The automation of the adaptive exposure time technique should also be researched. Currently the system adapts exposure times using Equation 1, which can only be used for the exact powder that it was calibrated for. Creating an automated adaptive exposure time technique would improve system usability and eradicate calibration requirements for new powders/materials. This would also remove the subjective nature of optimum exposure time selection.

To achieve high quality melt pool images, low exposure times and notch filters must be used. To achieve high quality melt pool images at a range of laser power settings, adaptive exposure times must be used. Increasing the quality of melt pool images has highlighted edge features that have not yet been documented in DED systems. Increasing the level of detail in melt pool images will allow for the development of new melt pool dimensioning algorithms. High quality melt pool imagery allows for newly developed algorithms to be directly compared with existing melt pool algorithms, and melt 
pool edge features, to determine dimensioning accuracy.

\subsection{Chapter Conclusion}

This research has contributed towards improving parameter monitoring systems in DED processes by using fundamental radiation mechanics to better understand melt pool imagery. Directional emittance is a phenomena that should been seen in coaxial melt pool images, showing dark regions around the edge of the melt pool, due to high surface angles. With this theoretical understanding, a qualitative analysis of previous literature was conducted, and it was found that no directional emittance features were apparent in any documented melt pool images. It was concluded that these images do not resolve the melt pool edges due to high image saturation caused by non-optimal machine vision techniques. Improving the machine vision techniques resulted in a greater subjective understanding of the process, and higher quality melt pool images.

Preliminary experimentation with camera settings allowed for a clearer view of the melt pool, highlighting directional emittance features. Although clearer images were achieved from the preliminary findings, it was noted that a single exposure time caused drastically different melt pool images for various laser power settings. In this study it was discovered that different exposure times need to be selected to achieve clear melt pool images at different laser power settings.

An in-depth experiment was conducted to distinguish the relationship between the exposure time and laser power settings. For laser powers between 600 and $1200 \mathrm{~W}$, low, best, and high exposure times were calculated. The results indicated a linear relationship between exposure time and laser power. Decreasing the exposure time linearly when increasing laser power allows for high quality melt pool images, with directional emittance features, at a range of laser power settings. The experiment provided a window of exposure times to operate within, and key melt pool features that could be used to calculate melt pool dimensions for all laser powers.

This chapter sought to optimise machine vision imaging techniques used in DED processes. A combination of optical optimisations and adaptive camera settings has allowed for the clear imaging of the melt pool in the DED process at a range of laser power settings. The optimisation of machine vision imaging techniques has provided a solid foundation for subsequent development of advanced 
melt pool dimensioning systems. Future work will utilise these advancements and image processing algorithms will be designed to measure melt pool characteristics with enhanced accuracy. 


\section{Algorithm Development}

\subsection{Introduction}

Achieving high quality images of the melt pool can allow for a better subjective understanding of the complex DED process, but more importantly it provides a solid foundation for image processing techniques to extract melt pool dimensions. Previous techniques have used sub-optimum quality images (as displayed in Figure 11), and image processing techniques that are not deemed to be of high accuracy. The most commonly used technique for conducting melt pool measurements uses a binarisation method, with a user defined threshold to produce a binary image whose feature size is representative of that of the molten metal area. This binarisation threshold is usually dictated by corresponding a pixel value that is representative of the melting temperature of the material using an emissivity value. Pixels above this threshold are deemed to be molten metal and pixels below are deemed to be substrate. This binary image is then subject to image processing techniques to extrapolate the dimensions of the melt pool. This threshold technique has been used to calculate melt pool dimensions in a range of different parameter experiments and for the implementation of closed loop feedback systems in AM processes [10, 24, 27, 30-34, 43-46, 60, 86, 87].

This chapter improves upon current melt pool image processing techniques by developing a new algorithm that detects edge features. The new algorithm more accurately measures melt pool edges when being directly compared to the conventional melt pool image processing technique. This chapter explains why the conventional image processing techniques cannot be used to accurately measure melt pool width.

\subsubsection{Conventional Melt Pool Dimensioning Techniques}

The binary threshold approach is the most commonly used method to measure melt pool dimensions. Of the papers referenced in this work, only the following discuss image processing techniques used for melt pool dimensioning in any detail $[10,24,30,32,34,43-46,60,87]$. Most of the literature utilises the same technique to calculate the dimensions of the melt pool, which can be broken down into the 
following steps:

1. Acquire an 8-bit image of the melt pool.

2. Perform binarisation on the image with a given user defined threshold.

3. Perform erosion on the image to reduce the amount of noise in the image.

4. Perform dilation on the image to increase the size of the melt pool to the same as it was before the erosion process.

5. Use the Canny edge detection method to outline all the remaining features in the image.

6. Extract the vector of the largest object detected using the Canny technique (typically the melt pool).

7. Use the moments of the vectors to work out the Centre Of Mass (COM), length, and width of the melt pool.

Researchers from different organisations sometimes approach the melt pool dimensioning step with minor variances, but all use a user defined threshold to perform the binarisation process. The binarisation process uses an 8-bit grey scale image that consists of 256 different pixel intensity values, ranging from 0-255. Performing a binary threshold on the image requires a user to define a limit, in which all pixel values above the limit become fully saturated (255) and all pixel values below the limit become fully unsaturated (0). After this stage there are only pure black or white pixel values in the remaining image.

In many of the publications referenced in Chapter 2, the authors state that a threshold is used, but do not explain how this threshold is chosen. The value of this threshold is arguably the single most influential value within the image processing algorithm, and directly influences the measured size of the melt pool. Increasing the threshold limit will decrease the size of the melt pool in the binary image leading to smaller measurements. In the following papers the threshold limit is discussed in more detail and it is chosen based on the pixel intensity related to either the liquidus-solidus or melting temperature for the given material $[24,46,60]$. Relating the pixel intensity value to the liquidus-solidus temperature 
requires an emissivity value for the material. Calculating the exact emissivity values for molten metals is complex and many fundamental thermographic concepts are not considered when values are used in documented melt pool monitoring systems. The complexity of non-contact temperature calculations and their tendency to cause inaccuracies in melt pool measurements is documented in the following papers $[39,40,47]$.

The accurate calculation of an emissivity value requires the user to construct a black body out of the material that is to be measured. This is something that is incredibly difficult with present day technology for molten metals, as a black body is a conceptual solid shape and forming this out of liquid metal proves difficult. Small fluctuations in emissivity values used for this calculation will cause a rise in melt pool dimension inaccuracies.

Even if a highly accurate emissivity value can be created for a molten material, this approach does not consider the variations of thermal radiation, in a given direction, caused by different surface angles. The effect that directional emittance has on thermal radiation emitting in a given direction from a surface is discussed in detail in Chapter 3.

Another issue with the current threshold-based image processing technique is the constant need for recalibration with different materials. The technique not only produces inaccurate results over a range of laser power settings, but also requires calibration for all new materials that are used in DED processes.

\subsection{Development of New Algorithm}

The inaccuracies of this commonly used melt pool dimensioning technique focuses this research into creating a more accurate melt pool monitoring system. Advanced melt pool monitoring systems can improve DED processes by providing a better understanding of the complex process and can allow for more accurate control systems. Improving this technique will also allow for a more in-depth parameter correlation study to be conducted, which will provide valuable information about how process variables effect melt pool geometry.

The new algorithm that is developed allows for as much diversification as possible. One of the major advantages of AM is the ability to rapidly produce components with new geometries out of 
new materials. Materials within a hopper on a DED system can change regularly in a industrial environment. The newly developed algorithm should not only perform melt pool measurements over a range of different temperatures but should possess the ability to be used on different materials with little to no calibration. The algorithm was designed using off-line video data that was acquired in the experimental procedures in Chapter 3.

\subsubsection{Frame Averaging}

\subsubsection{Requirements and Observations}

In the development of the new image processing algorithm, frame averaging takes a major role in creating a solid and stable image of the melt pool. Images with low exposure times capture the melt pool radiation over very short periods, allowing for the acquisition of images with intricate melt pool details. Whilst these details can be advantageous for certain situations, when trying to perform feature extraction on the melt pool they can cloud key melt pool characteristics such as the COM and melt pool width.

When attempting to calculate the COM location (Section 4.2.2 discusses this algorithm in detail), issues arose when the algorithm was performed on a single raw image. This is due to a combination of the highly erratic DED process and the low exposure time setting capturing melt pool radiation over a short time period.

In early algorithm development it was noted that imaging the melt pool at such small time intervals showed inconsistencies in radiation emitting from the melt pool's top surface over a number of subsequent frames. In nearly all frames, there is a region of saturated pixels near the centre of the melt pool that indicates a combination of high thermal radiation and low surface angles. This region of saturated pixels changes both its size and location throughout different frames in the video. This can be seen in Figure 30, where adjacent images are taken from a single deposition recording.

The change in location of this saturated region, indicates that the melt pool surface is changing. The shape of the melt pool cannot be simply approximated as a half sphere. This is due to interactions from physical forces causing distortion during the process including powder, carrier gas, and shielding gas injection. Thermal interactions, such as thermal gradients caused by laser movement, may also 

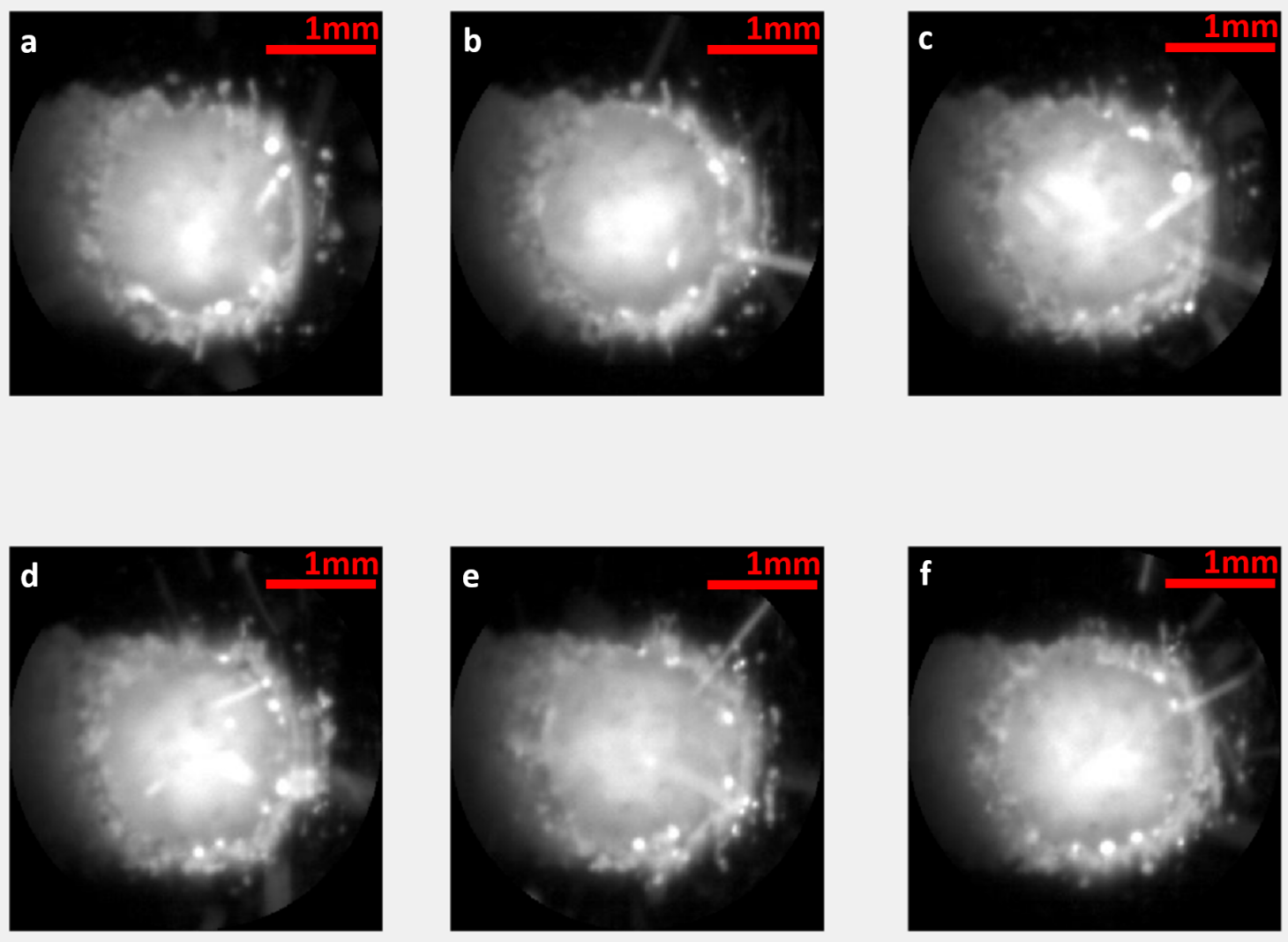

Figure 30: A series of images displaying the change in size and location of the high intensity pixel region at the centre of the melt pool for adjacent frames (exposure time - $0.55 \mathrm{~ms}$, laser power $600 \mathrm{~W})$.

have an influence on the shape of the melt pool. Thermal gradients are also present between solid powder particles and molten metal within the melt pool itself. The complex interactions of these forces with the melt pool would suggest that its surface would not behave identically to a fluid simply under cohesion and abhesion forces. These additional forces are likely to cause fluctuations in the melt pools surface, which would be more evident in images taken with low exposure times. Low exposure time imaging is often used to capture fast moving objects, as the radiation blurs over a smaller period of time. This is why previously documented melt pool images that use longer exposure times see a more 
even distribution of thermal radiation (displayed in Figure 11).

Another issue that occurs when acquiring images with such low exposure times is the large amount of noise present. Figure 30 not only shows the change in size and location of the high intensity pixel region, but also shows two phenomena in the AM process that cloud the edge of the melt pool. The edge of the melt pool is often clouded by un-melted particles just entering the melt pool causing bright spots of radiation, and powder particles with high radiation travelling across the FOV of the CMOS sensor.

At the front edge of the melt pool there are often bright spots that can cause issues when trying to create an image processing algorithm to detect the melt pool edge. These regions of high intensity radiation are thought to be a cause of powder particles entering the melt pool at the specific point in time when the image is acquired. Powder particles that are in contact with the high temperature melt pool region, that are yet to enter the molten material, would likely give off high amounts of radiation in the direction of the camera. This is due to part of their spherical surface emitting maximum radiation in the direction of the CMOS sensor. This theory is displayed using a schematic in Figure 31a.

Figure $31 \mathrm{~b}$ shows a still image with these bright spots present, and several red arrows highlight these features. Interestingly the bright regions only occur around the front of the melt pool, which could be explained by the thermal gradient present resulting in the front of the melt pool being of higher temperature than the rear. This would also explain why there are dark regions in the tail of the melt pool, which may be an indication of un-melted powder particles not having enough energy to completely melt. Powder particles at the tail of the melt pool would be in contact with a surface that has a low surface angle, meaning that the contrast in radiation due to the phenomena of directional emittance is greatly reduced.

Another contributing factor to the bright regions only being located around front edge of the melt pool could be the velocity that the powder particles are travelling at. Powder particles are injected into the melt pool using a carrier gas. Powder particles that are travelling to the centre of the melt pool are likely to have a higher velocity than those that have drifted away from the direction of the projected gas. If a powder particle is travelling at a lower velocity, it is less likely to break the surface tension of the molten material, and more likely to be caught in a single instance of a CMOS image 
capture.
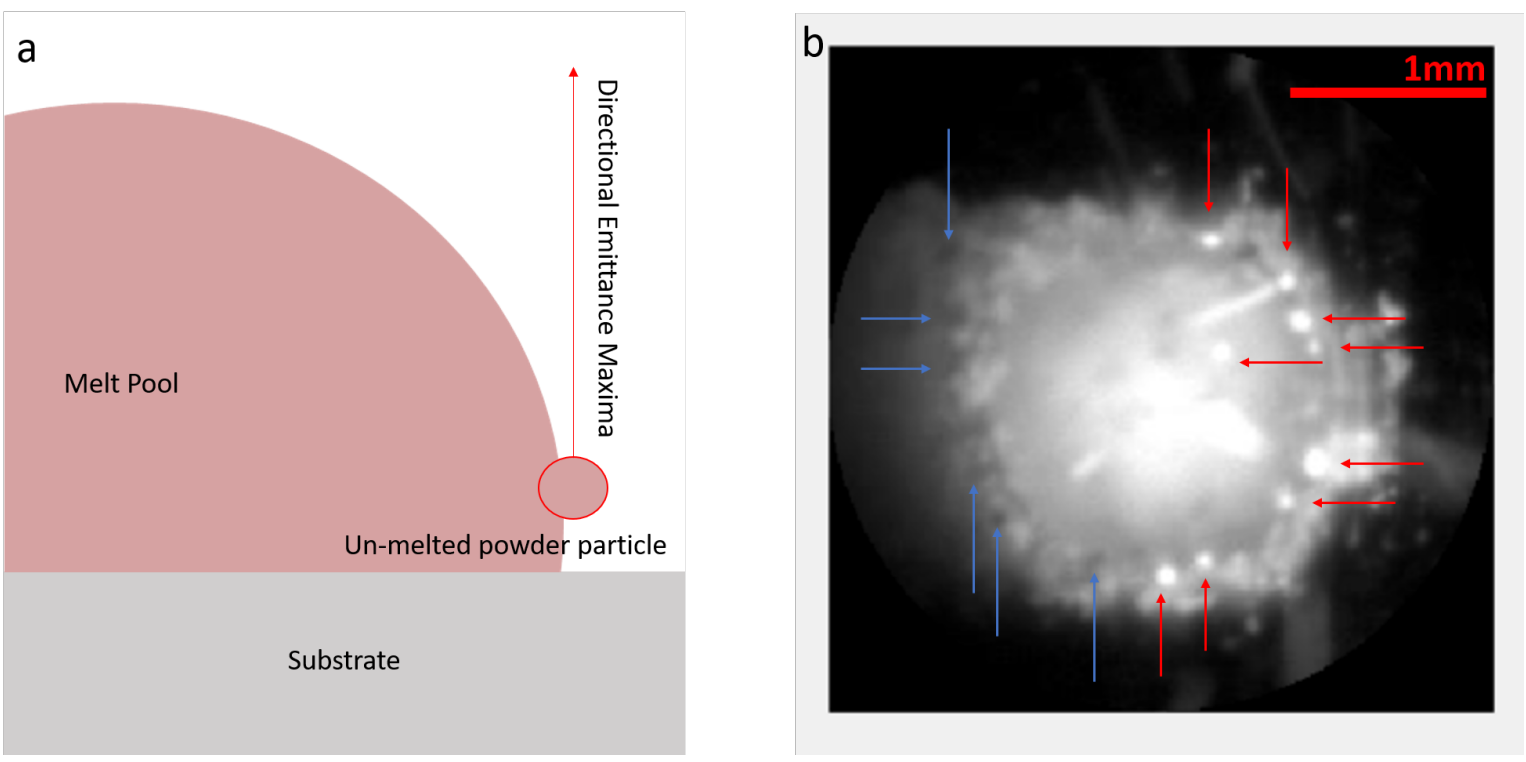

Figure 31: A single frame taken from a melt pool video recording deposition at $600 \mathrm{~W}$ with an exposure time of $0.55 \mathrm{~ms}$. This image highlights bright regions around the edge and in the centre of the melt pool.

Whilst these un-melted powder particles cause issues with image processing and melt pool dimensioning, they are discussed in detail due to the prospect of their study in the future. The number of these particles could be measured using another image processing technique to provide an indication of the percentage of powder that is being absorbed by the melt pool. Low power and high PMFRs are likely to cause a rise in these bright and dark regions, which could be used to provide a powder absorption efficiency of the melt pool. This efficiency could subsequently be used to tailor the input parameters to reduce the amount of wasted powder in AM processes.

High speed powder particles that travel across the CMOS sensors FOV during capture cause long strips of saturated pixels across the melt pool image. These regions of saturated pixels can cloud image processing techniques and give false readings, especially when they cross the melt pool's front surface. These flares are depicted in Figure 32. Both un-melted powder particles and bright flares cause issues 
when trying to extract melt pool dimensions from a melt pool image.

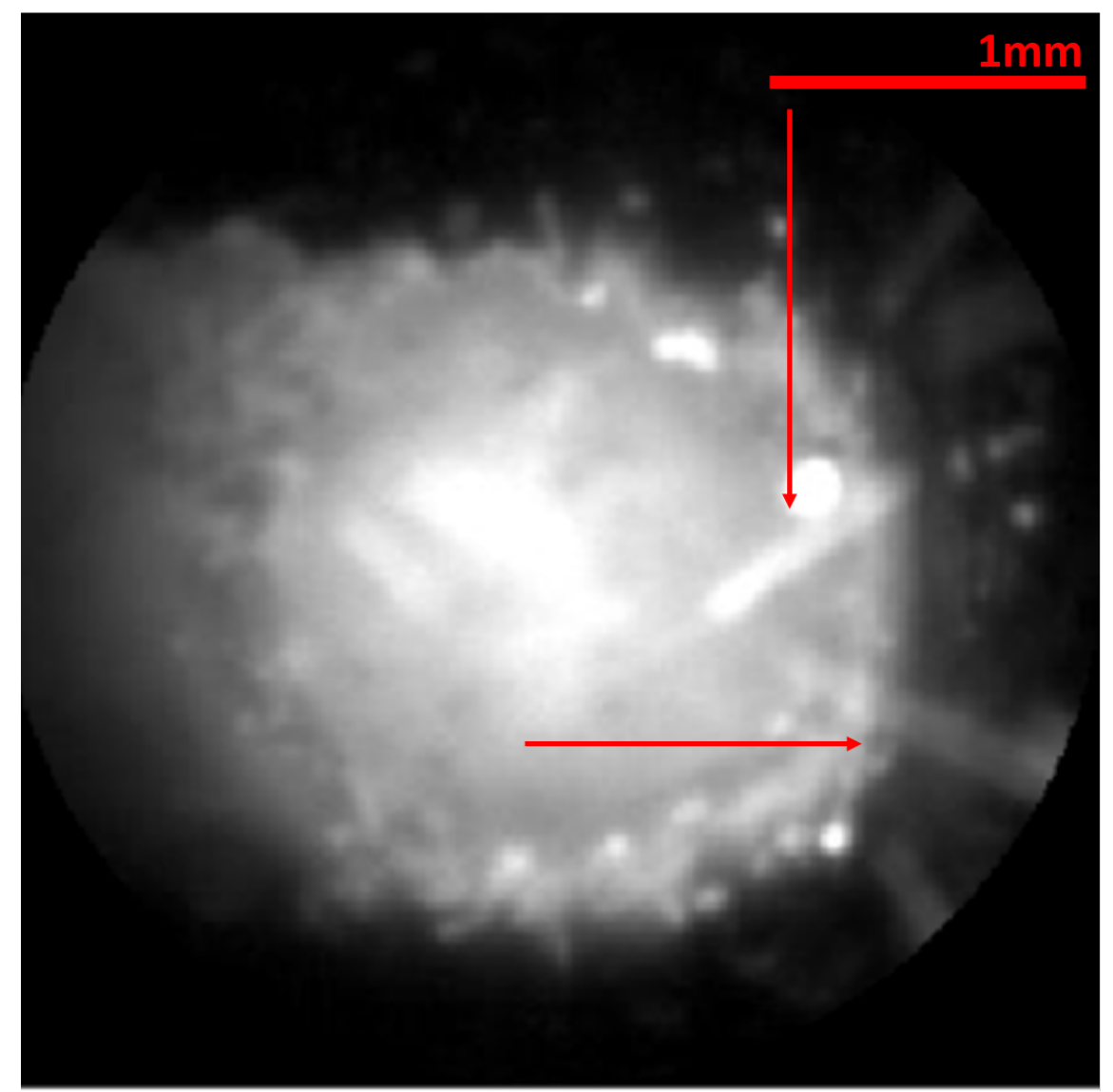

Figure 32: A single frame taken from a melt pool video recording deposition at $600 \mathrm{~W}$ with an exposure time of $0.55 \mathrm{~ms}$. This image highlights long strips of saturated pixels caused by high speed powder particles.

\subsubsection{Method}

To increase the stability of the image's COM and reduce the contribution of noise, frame averaging is carried out on the image feed. This technique requires a video feed with multiple frames and works by adding the pixel intensities (at each given pixel location) across the user defined number of frames to an image holder. The resulting summations are then divided by the total number of frames that have 
been averaged across. Equation 2 shows the mathematical formulation for this.

$$
P_{x y}=\frac{1}{n} \sum_{i=1}^{n} a_{i}
$$

Where $P_{x y}$ is the average pixel value at a given $x, y$ location in the averaged melt pool image, $n$ is the number of frames averaged across, and $a$ is the pixel value at a given $x, y$ location in melt pool image $i$.

The frame averaging technique is performed using frames that have been previously recorded, meaning that all frames before frame number $n$ have slightly different equations. Equation 3 shows the mathematical formulation for this.

$$
P_{x y}=\frac{1}{m} \sum_{i=1}^{m} a_{i}
$$

Where $m$ is the number of frames captured.

The transition of this initial stage (changing from Equation 3 to Equation 2 ) is displayed in Figure 33. Performing the frame averaging technique in this manner allows for the improvement of melt pool image quality without sacrificing frame rate.

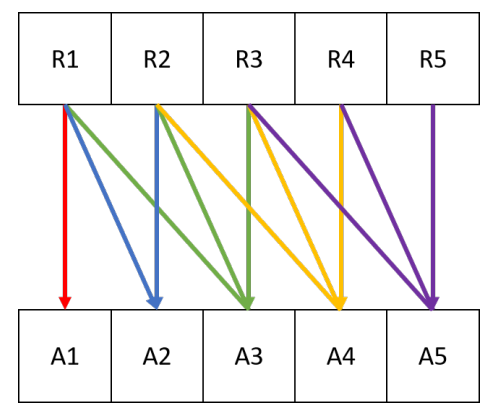

Figure 33: A graphical representation of how the initial frames contribute to the averaging of frames. This diagram displays how raw images contribute to average images using a averaging window width of three. 


\subsubsection{Result}

The result from averaging the melt pool images shows a drastic reduction in the amount of noise, and a more even distribution of highly saturated pixels in the centre of the melt pool. By averaging over a period of time, the noise caused by un-melted and high speed powder particles was greatly reduced, as their contribution to the averaged image is a fraction of what it is in its original image. Features that are not constant throughout the different frames are reduced, and features that stay relatively constant (including the melt pool's edge) are highlighted. The averaging algorithm that was mentioned in 4.2.1.2 was performed on the images displayed in Figure 30 to produce the images in Figure 34.
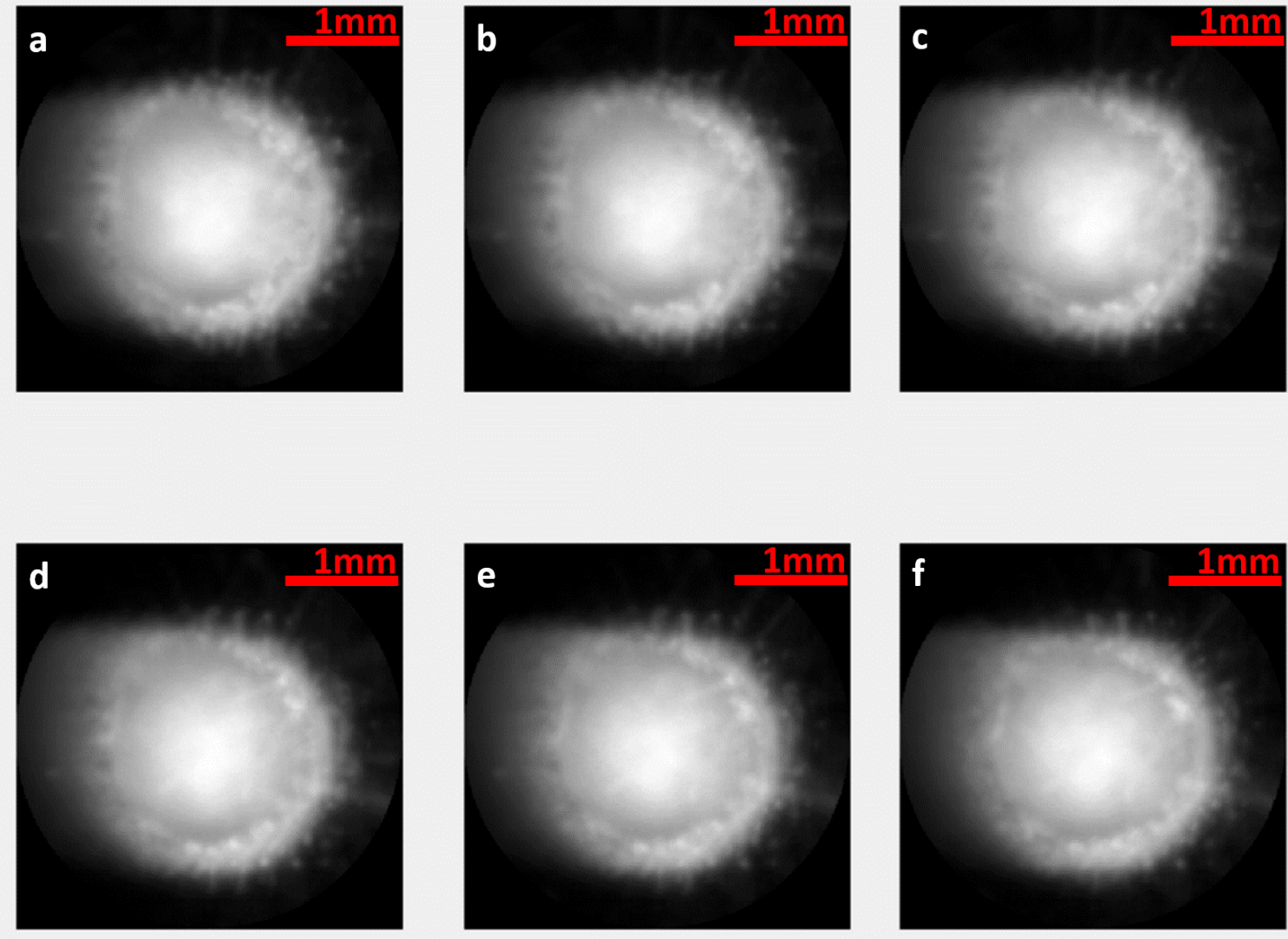

Figure 34: A series of melt pool images that have been subject to the frame averaging technique. 
The averaging technique was applied on the following images with an averaging window $(n)$ of seven. This value provided images with low noise and clear melt pool edges. Averaging a melt pool across multiple images has both advantages and disadvantages. One disadvantage is that this method can detract information from the images that are acquired. This algorithm is developed to extract the width and $\mathrm{COM}$ of the melt pool and by creating an average window we reduce the resolution over time. This said, most control systems that use melt pool dimensioning algorithms utilise the dimensions to counteract issues like heat accumulation. The time scale of heat accumulation is something that is magnitudes longer than the resolution lost over the method of frame averaging, meaning that frame averaging will have little to no effect in controlling process parameters over entire builds.

When $n$ is increased further, it reduces the prominence of certain features that are of importance. The edge of the melt pool, highlighted by the directional emittance feature, becomes blurred and less prominent if the value of $n$ is increased too much. Throughout the DED process when using a pulsed laser, the melt pool will expand and contract due to the cycling of the delivered power. This expansion and contraction changes the location of the directional emittance feature and results in the average image not having an as distinguishable dark region. This subsequently causes difficulty when trying to implement an image processing algorithm to extract melt pool dimensions. Figure 35 displays averaged images of the melt pool using different values of $n$.

The location of the COM was tracked for a series of fifty frames using both the raw image feed and the developed averaging technique. Figure 36 shows that averaging the melt pool using a value of $n=7$ provides much more stability in the COM measurement. The dimensions of the melt pool images used for these calculations were $300 \times 300$ pixels. The images of averaged melt pools in Figure 34 show a much more homogeneous distribution of highly saturated pixels in the centre of the melt pool. This increase in stability when calculating the melt pool's COM will prove useful when trying to develop control systems that can monitor large changes in the COM location over the course of entire builds. 

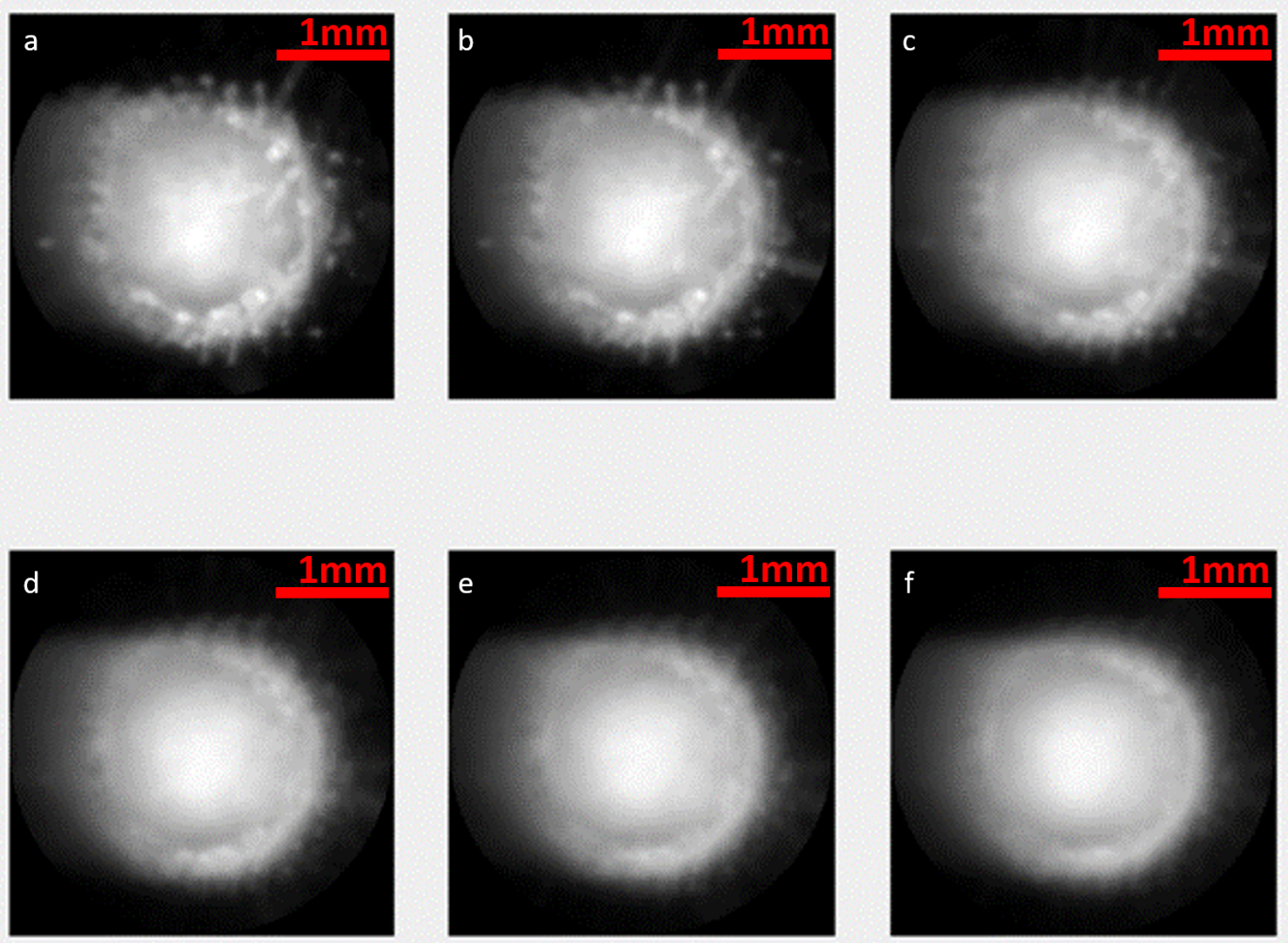

Figure 35: Images displaying the effects of varying $n$ for the averaging of melt pool images. a) $n=2$, b) $\mathrm{n}=3$, c) $\mathrm{n}=7$, d) $\mathrm{n}=10$, e) $\mathrm{n}=15, \mathrm{f}) \mathrm{n}=25$. 


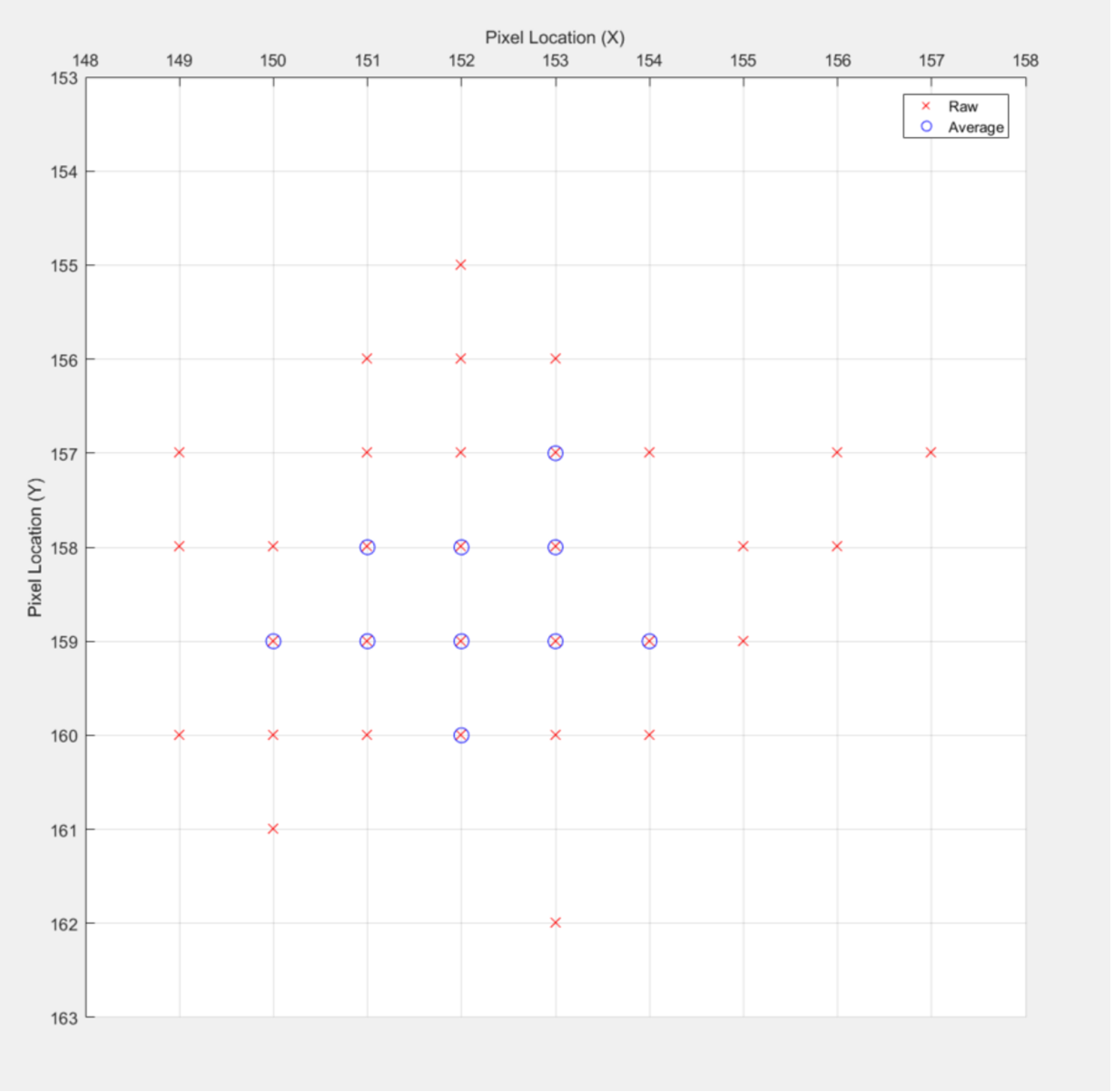

Figure 36: Centre of mass locations over fifty sample frames using both the raw image feed and averaged image feed.

\subsubsection{Conclusion}

Performing frame averaging on the raw image feed has allowed for a stabilisation of the melt pool image's COM, and eradicated features such as high speed and un-melted powder particles. In doing so this has allowed for the development of a new image processing algorithm that can measure the melt pool's width based on the directional emittance phenomena. 


\subsubsection{Centre of Mass Calculation}

Calculating the $\mathrm{COM}$ of the melt pool image can provide both valuable information for the development of control systems, whilst simultaneously providing a starting point for the melt pool width extraction algorithm. Large shifts in the COM could provide indications that the AM build process is not performing in the way that it should, for example when printing overhangs or thin wall structures.

Once an image is acquired that has been subject to frame averaging, the image processing algorithm designed can start the first stages of calculation. The calculation of the COM uses a combination of reduction and scan techniques.

There are two reduction calculations that occur when resolving the COM, one along the length of the columns (X direction) and one along the length of the rows (Y direction). The reduction technique along a given direction adds up all the pixel values in either a given row or column to an array that stores all of these values. Equation 4 shows the mathematical formula for the reduction of all columns. Equation 5 shows the mathematical formula for the reduction of all rows.

$$
R_{x}=\sum_{y=1}^{Y} a_{x, y}
$$

Where $R_{x}$ represents the reduction value for a given column $(x)$ in image $a, y$ represents the column number, $Y$ represents the maximum number of columns, and $a_{x, y}$ represents a pixel at the given $x, y$ location.

$$
R_{y}=\sum_{x=1}^{X} a_{x, y}
$$

Where $R_{y}$ represents the reduction value for a given row $(y)$ in image $a, x$ represents the row number and $X$ represents the maximum number of rows.

Once the two stage reduction process is completed, there are two arrays, one with length $X$ and one with length $Y$. These arrays have fully reduced columns and rows, for all rows and columns respectively. These calculated arrays are represented in Figure 37. 

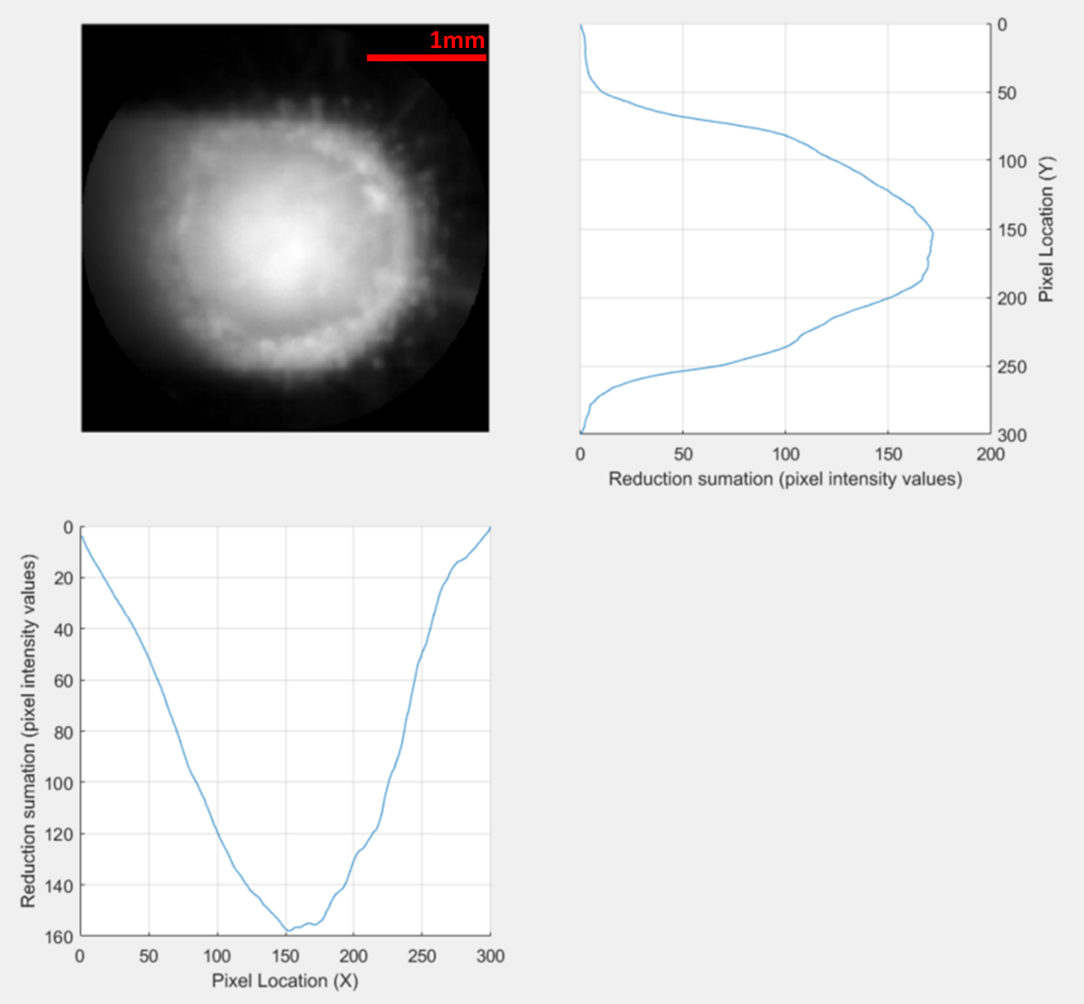

Figure 37: A graphical representation of the reduction of columns and rows for the averaged melt pool image.

The next stage in the calculation of the melt pool's COM uses an inclusive scan operation to alter the reduced arrays. The scan method iterates through the original array, and adds all the elements preceding (and including, due to the scan being inclusive) its iteration position until it reaches the end of the array. This can be thought of as a more complex reduction technique, where at any given point in the array, $(i)$ is a reduction of the elements that precede it $(1-i)$. The mathematical formula for the scan operation on an array is denoted by the following equation.

$$
S_{N}=\sum_{n=1}^{N} R_{n}
$$

Where $S_{N}$ is the resultant scan array with total length $N$, and $R$ is the input array subject to the 
cumulative scan summation.

Using Equation 6 on the two reduction arrays depicted in Figure 37 scan distributions in $\mathrm{X}$ and $\mathrm{Y}$ directions for the melt pool image can be achieved. The two scan distributions are depicted in Figure 38.
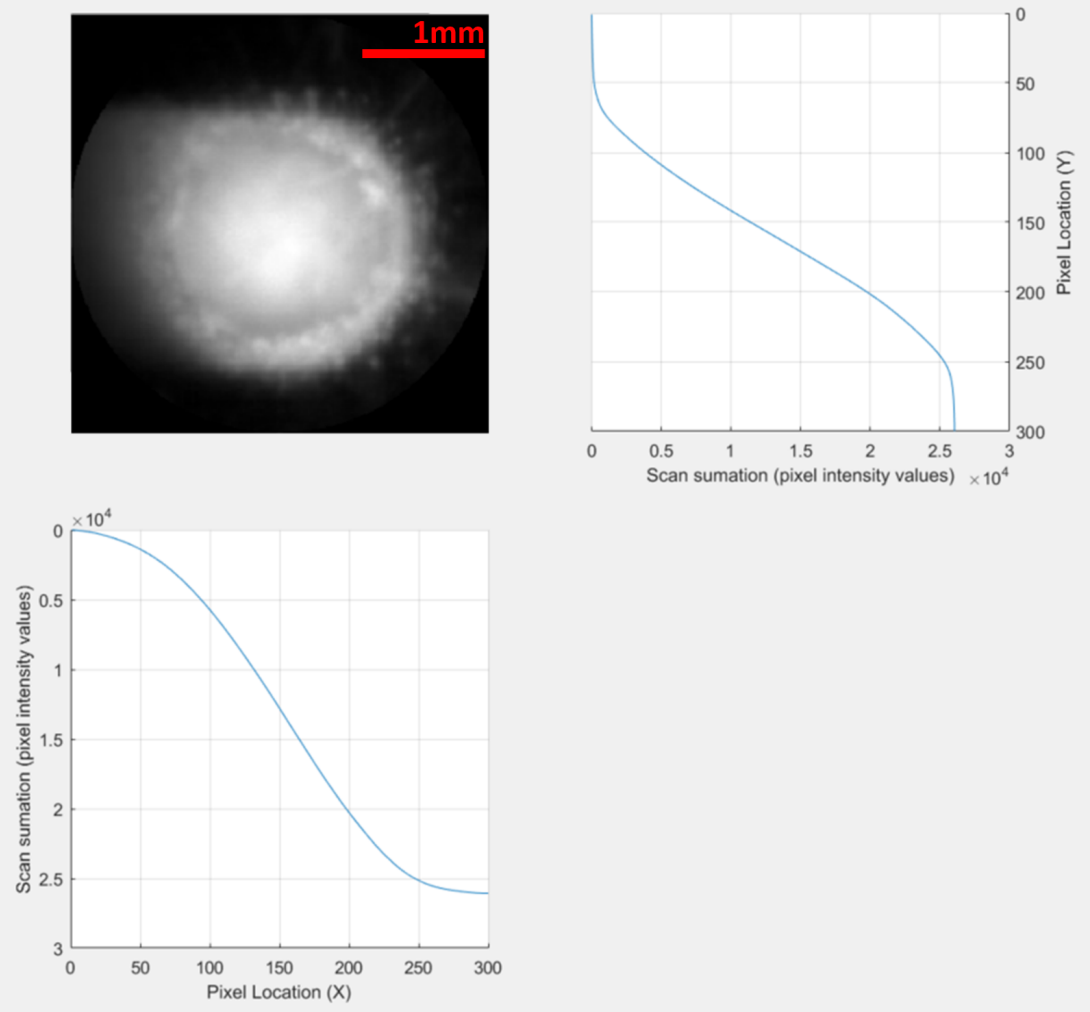

Figure 38: A graphical representation of the scan arrays that were achieved using the reductions arrays subject to Equation 6

From these results we can extrapolate the location of the COM in both the $\mathrm{X}$ and $\mathrm{Y}$ directions. The scan summation in itself has provided the total saturation level of all pixels in the image. This saturation level can then be halved, to find the COM saturation value in both the $\mathrm{X}$ and $\mathrm{Y}$ directions. Finally, an iteration is performed on the relevant scan arrays to find out the location of the COM in 
both the $\mathrm{X}$ and $\mathrm{Y}$ directions. The result for the COM using this method is depicted in Figure 39. The equations for calculating the COM location with regards to the scan arrays produced using Equation 6 are denoted in Equations 7 and 8.

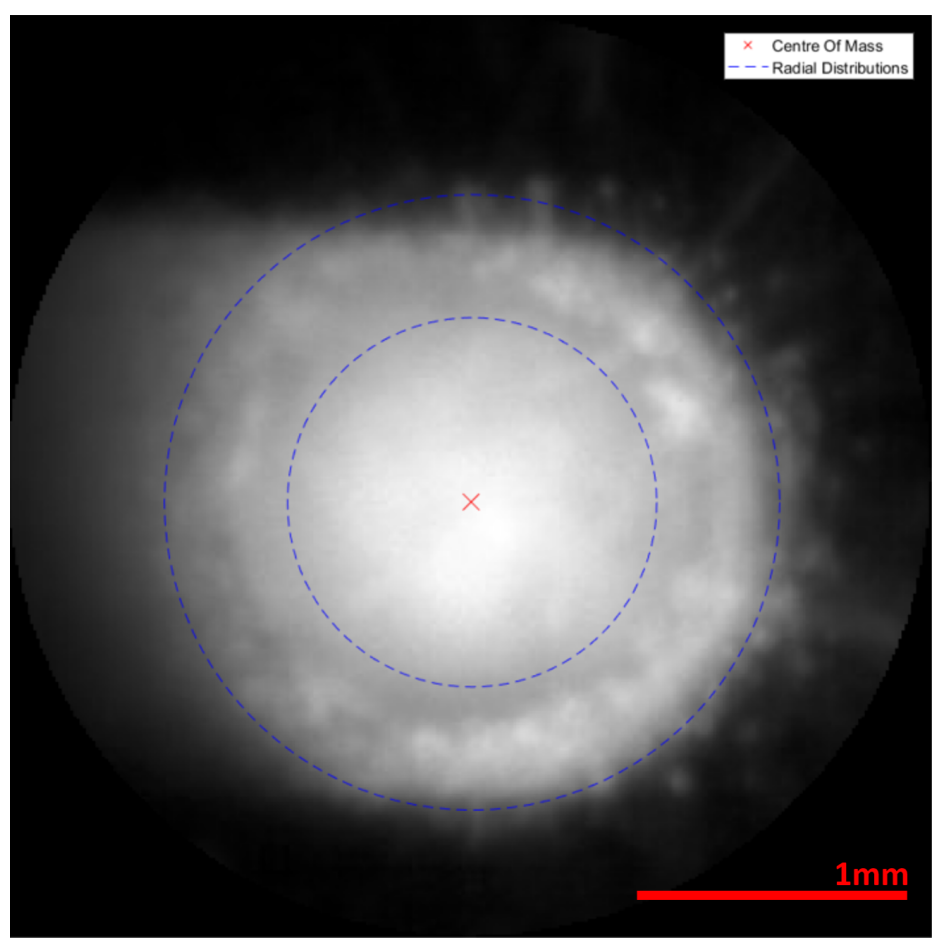

Figure 39: An image of the melt pool with the calculated COM. Radial distributions have been added to more easily distinguish equal distances from the COM location.

$$
\begin{aligned}
& C_{x}=i \quad \text { where } \quad S_{i} \leq \frac{S_{X}}{2}<S_{i+1} \\
& C_{y}=i \quad \text { where } \quad S_{i} \leq \frac{S_{Y}}{2}<S_{i+1}
\end{aligned}
$$

This method has been successfully used to give an approximate location of the centre of the melt pool. Calculating the COM of an image does not necessarily mean the calculation of the centre of the melt pool, but without known values of the melt pool's dimensions the calculation of the melt 
pool centre would be very difficult. The DED process uses a focused source of energy that results in the image showing a radial decay of energy from the centre. Figure 37 shows this phenomena after the reduction of all pixels in both the $\mathrm{X}$ and $\mathrm{Y}$ direction, by displaying two distributions that show strong correlation to a Gaussian distribution. Due to the high amount of symmetry in both the $\mathrm{X}$ and $\mathrm{Y}$ directions this approximation can calculate a position in the image that lies both within the molten metal, and that has relatively even distances to the directional emittance phenomena in multiple directions. This approximation is the anchor point for the subsequent stage in the algorithm that detects the edges of the melt pool.

\subsubsection{Edge Detection}

The calculation of the COM has provided a crucial starting point for melt pool edge detection. Using this approximate centre, and knowing the direction of travel, the edge detection technique can project its readings in multiple directions to calculate melt pool dimensions. The edge detection technique works by calculating two points on the melt pool's surface, in which a subsequent width dimension can be extracted.

\subsubsection{North and South Locations}

The names given to the melt pool edge locations for ease of explanation will be 'North' and 'South' points. The locations of the north and south points are displayed in Figure 40. Calculating the north and south points of the melt pool allows for the calculation of melt pool width. 


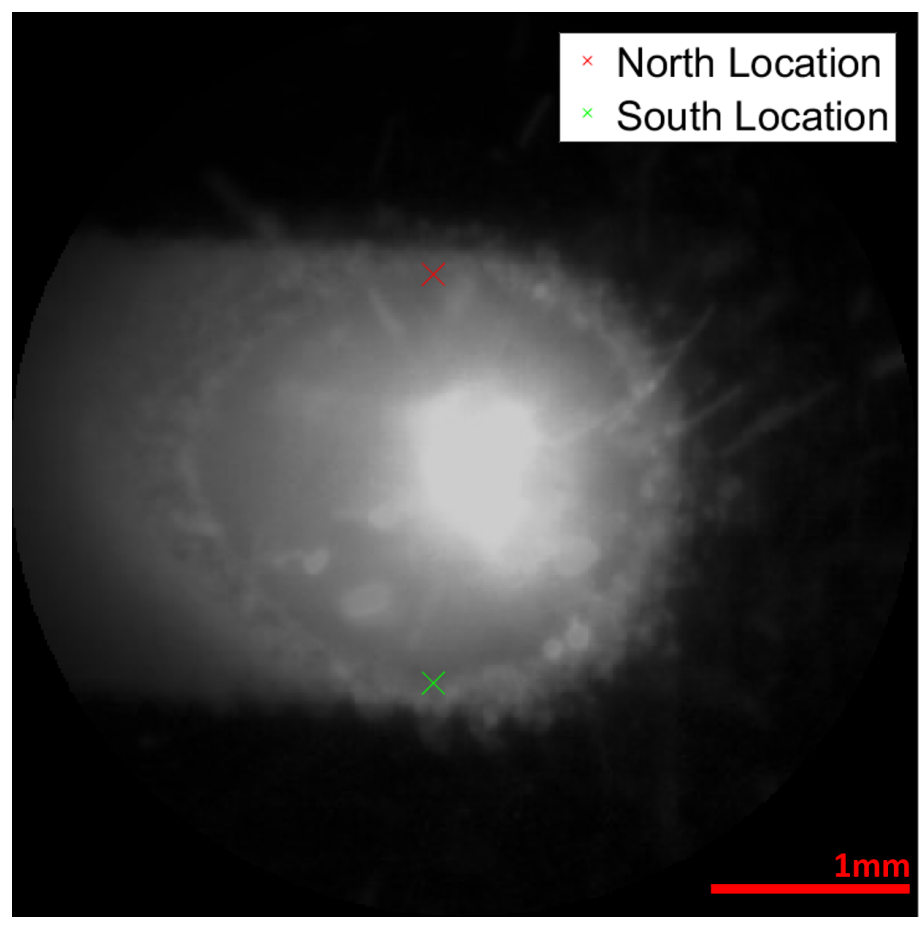

Figure 40: An averaged image of the melt pool showing the north and south locations.

\subsubsection{Raw Signal}

Edge detection algorithms are performed on a set of columns extracted from the melt pool image. The extracted column from a melt pool image is referred to as a signal for ease of explanation in this research. Figure 41 displays a signal extracted from the melt pool image whose location is at the calculated $C_{x}$. This signal using the single column located at the $C_{x}$ is referred to as the raw signal. Dotted lines are used in this diagram to allow for easy viewing of the underlying signal/melt pool image. Dotted lines throughout this research are merely used as a graphical tool to better present data.

The raw signal shows some key characteristics that are representative of the melt pool features. There are high saturation levels in the centre of the signal that show strong radiation levels emitting from the centre of the melt pool. Moving from the centre, a decay in radiation is observed as the pixel 

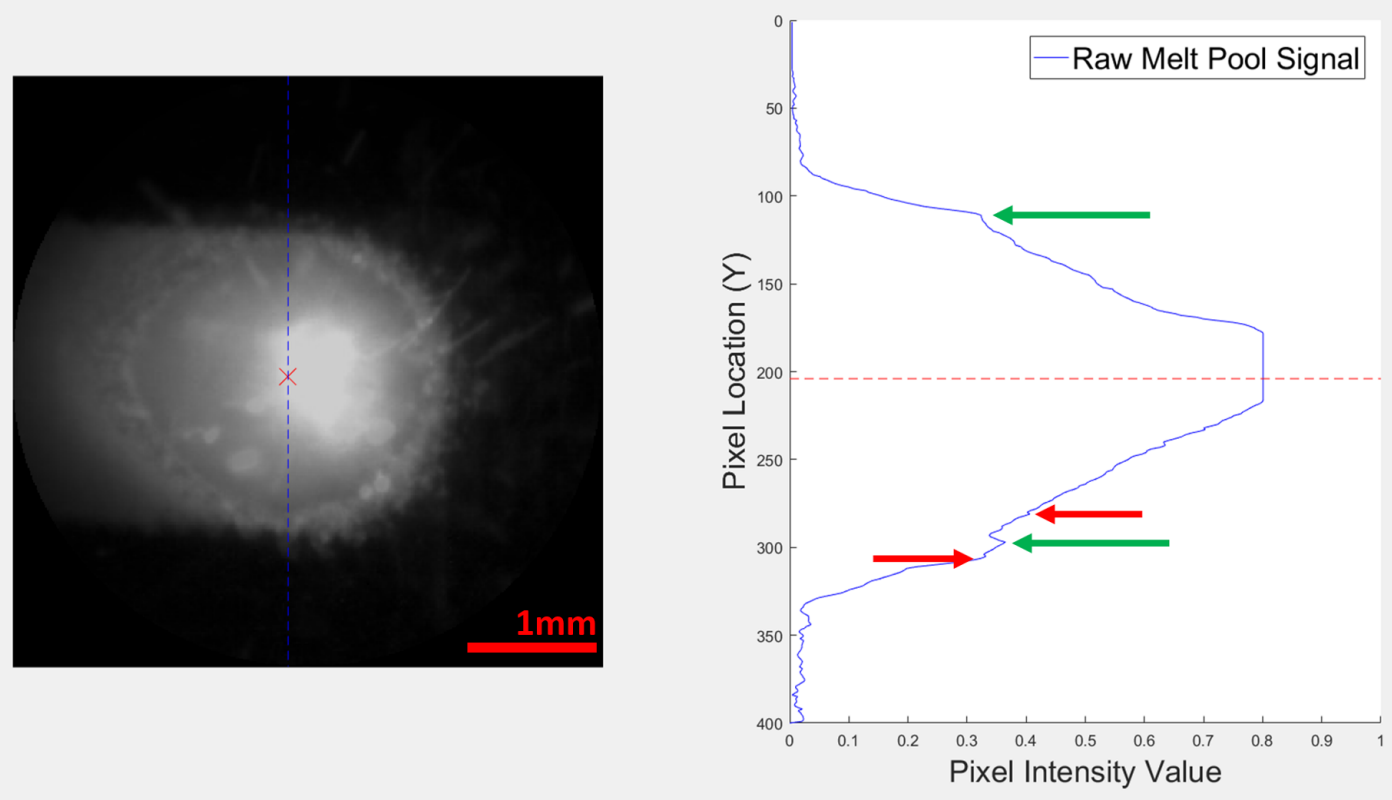

Figure 41: Left: an image of the melt pool after frame averaging has occurred. Right: The raw signal that is extracted from the melt pool along the row at the COM-Y location.

locations are moving away from the central energy source. The movement also sees a reduction in the melt pool angles relative to the CMOS sensor. Distinct features in the signal are highlighted by the green arrows, where the trend in reducing pixel saturation values is broken by a sudden rise in radiation. This feature in the extracted raw signal indicates the directional emittance feature surrounding the edge of the melt pool. The kink in the signal indicates that the surrounded solid substrate emits radiation at a higher intensity than the edge of the melt pool surface. It is believed that this feature in the signal is directly related to the directional emittance phenomena and subsequently the edge of the melt pool. This feature will be used to extrapolate melt pool dimensions.

Whilst the section of the signal, represented by the green arrow, shows a sudden increase in pixel intensity value, there are also other parts of the signal that show an increase. The red arrows in this diagram highlight other regions of the signal where there are sudden changes in pixel intensity values, which could give false readings when calculating the melt pool dimensions. These signals are due to 
noise in the melt pool image, and whilst frame averaging has benefited this signal greatly, the raw signal extracted from the melt pool image still shows noise that is detrimental to the detection of edges. For this reason, further averaging and smoothing is to be carried out, so that the directional emittance feature can be extracted. The raw signal displayed here is not used in the calculation of the north and south locations. It was merely used in the discovery of the directional emittance feature.

\subsubsection{Averaged Signal}

To make the directional emittance feature more distinguishable, filtering methods are used prior to edge detection. Rather than extracting a single line at the calculated $C_{x}$, a narrow band of signals are extracted using a window evenly surrounding the $C_{x}$ location. This window is referred to as the average window. White spots can often land in the dark region surrounding the melt pool at the COM-X location, so by taking an averaging window rather than a singular line, the contribution of random white spots is reduced. The size of the window is kept small in comparison to the size of the melt pool, resulting in the summation of eleven rows (five either side of the COM-X location). The window size of five was selected as lower values caused instability in the calculations and higher values altered the calculated location. Variations are negligible due to the small size of the window and the symmetrical distribution of averaging. An example of the averaged melt pool signal is displayed in

Figure 42. The mathematical formula to calculate the averaged signal is described in Equation 9.

$$
A_{y}=\frac{1}{2 j+1} \sum_{x=C_{x}-j}^{C_{x}+j} a_{x, y}
$$

Where $A_{y}$ is the average signal, $2 j+1$ is the size of the average window, $C_{x}$ is the COM in the $x$ direction, and $a$ is the averaged image.

\subsubsection{Smoothed Signal}

The averaged signal is then subject to a second stage which utilises a smoothing function to remove/reduce remaining noise in the signal. The signal generated from using this equation is referred to as the smoothed signal. Care must be taken when performing this smoothing function on the signal 
as to not shift the signal in a certain direction. Common signal processing functions such as the moving average function (also known as the central moving average function) can be used. This function uses an equal number of data points either side of a central value, calculates the mean using these values, and then replaces the central value with the calculated mean. This technique requires an odd number of total data points in the resulting calculation to eradicate data shifting. The equation for the smoothing function used in the calculation of the north and south melt pool locations is displayed in Equations 10 and 11. The smoothing window size used for this signal was seven. This window size was selected as values below were unable to reduce noise effectively, and values above began to detract from the key directional emittance features.

$$
T_{y}=\frac{1}{2 k+1} \sum_{j=-k}^{k} A_{y+j} \quad \text { when } \quad y=k+1, k+2, \ldots, Y-k
$$

or

$$
T_{y}=A_{y} \quad \text { when } \quad y \leq k \text { and } \quad Y-k<y \leq Y \text {. }
$$

Where $T_{y}$ is the smoothed signal, $2 k+1$ is the size of the smoothed window, $y$ is the location of the cell in the array and $Y$ is the length of the array.

The raw signal, averaged signal and smoothed signal are displayed next to each other in Figure 42 . 

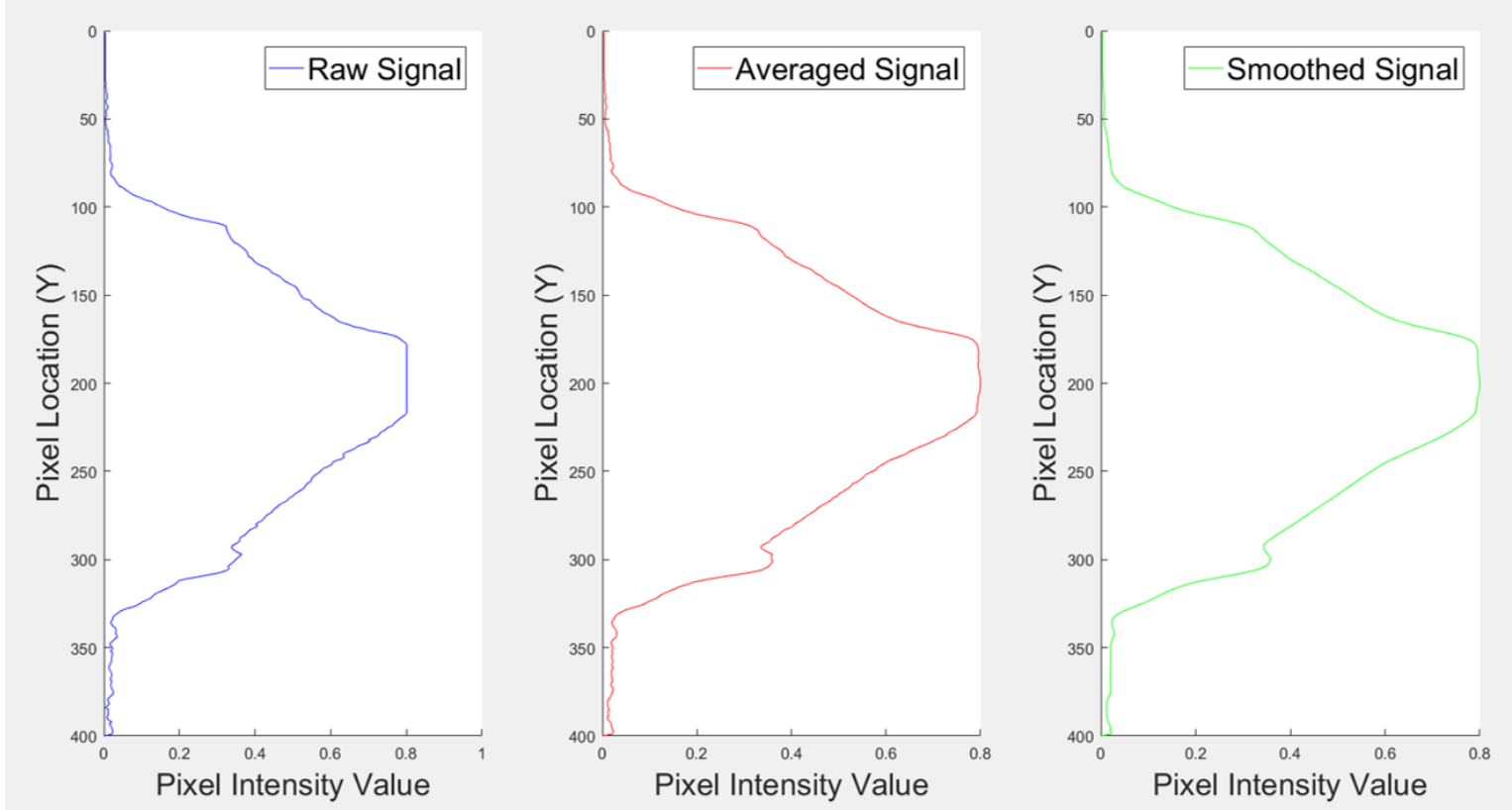

Figure 42: Three graphs displaying the transition between the raw signal, averaged signal, and smoothed signal

All plots in Figure 42 show changes in gradient at distances close to the melt pool edge location. These kinks can be linked to both the directional emittance phenomena and melt pool edge. The averaged and smoothed signals progressively eradicate noise in the signal to further highlight the key changes in gradient.

\subsubsection{Threshold Window}

The next stage in the developed algorithm is the application of thresholds to the signal. Applying a threshold to a signal can be a very effective way of extracting relevant information and filtering out parts that are not needed. The new smoothed signal shows a stable distribution of the melt pool's radiation through the $C_{x}$, but only a small portion of the signal is needed to detect the edge of the melt pool. Two threshold values are used on this signal to create a threshold window. The threshold window has both an upper and a lower threshold value, with the aim of the window being to capture a 
sample of the signal that contains the directional emittance phenomena. The method uses the natural decay of pixel intensity values as iterations move from $C_{x}$, with variables marking pixel locations when thresholds are broken. Two iterations are performed, one in the positive $\mathrm{Y}$ direction, and the other in the negative $\mathrm{Y}$ direction. The iterations compare all pixel intensity values to the upper threshold limit. When a pixel intensity values falls below the threshold limit, the pixel locations in which this occurred are stored. The iteration then proceeds until the lower threshold limits are broken. Again, the pixel locations at these conditions are stored. These upper and lower locations are used to extract a portion of data from the smoothed data signal.

The selection of upper and lower threshold values that are applicable for melt pool dimensioning at all laser powers is difficult. Due to the difference in intensity caused by images with varying exposure times, values for the upper and lower threshold limits were calculated based off the laser power setting used to deposit material. In short, the videos recorded on the lower laser power settings had the directional emittance phenomena occur at higher threshold levels. Threshold values of 0.7 and 0.4 for the upper and lower limits reliably encapsulate the directional emittance phenomena for most (if not all) of the frames in videos recording deposition at $600 \mathrm{~W}$. Increasing the laser power in the newly developed system resulted in a decrease in exposure time. One of the main reasons for doing so is to reduce the number of saturated pixel values in the centre of the melt pool, but this has the effect of reducing the intensity levels that the directional emittance phenomena resides around. Videos recording deposition at a laser power setting of $1200 \mathrm{~W}$ required lower threshold values of 0.5 and 0.3 for the upper and lower threshold limits respectively. The reason for this could be down to the methodology of experimental procedures in Chapter 3, and improvements on experimental design could produce video recordings that show greater stability of the directional emittance phenomena across different laser power settings. The need to change the threshold windows could however just be down to the nature of the melt pool. Increasing laser power not only increases the intensity of radiation received by the CMOS sensor but also increases the size of the melt pool. Increasing the size of the melt pool would increase the number of saturated pixels in a corresponding image due to a larger area within the FOV having low surface angles relative to the sensor.

To automate the selection of the threshold windows as much as possible for a given laser power 
setting, interpolation between the two extremes was conducted. Equations 12, 13, and 14 were created to allow for more autonomous melt pool measuring. These equations calculate a central intensity value $I_{c}$ and use two user defined threshold coefficients to locate the threshold window around the directional emittance phenomena. The user must therefore select an upper and lower threshold coefficient for the lowest and highest laser power settings that are to be subjected to the melt pool dimensioning algorithm. The lower and upper threshold limits are then calculated using Equations 12, 13 and 14.

$$
U=\left(U_{\max }-\left(\frac{P-P_{\min }}{P_{\max }-P_{\min }} \times\left(U_{\max }-U_{\min }\right)\right)\right) \times I_{c}
$$

Where $U$ is the upper threshold for a given laser power $(P), U_{\min }$ is the user defined upper threshold value at the lowest laser power setting, $U_{\max }$ is the user defined upper threshold value at the highest laser power setting, $I_{c}$ is the central intensity, and $P_{\max }$ and $P_{\min }$ are the maximum and minimum operating laser powers respectively.

$$
L=\left(L_{\max }-\left(\frac{P-P_{\min }}{P_{\max }-P_{\min }} \times\left(L_{\max }-L_{\min }\right)\right)\right) \times I_{c}
$$

Where $L$ is the lower threshold for a given laser power $(P), L_{\min }$ is the user defined lower threshold value at the lowest laser power setting, $L_{\max }$ is the user defined lower threshold value at the highest laser power setting.

and:

$$
I_{c}=\frac{1}{2 m+1} \sum_{n=C_{y}-m}^{C_{y}+m} T_{y}
$$

Where $T_{y}$ is a cell in the smoothed signal at a given location $y . C_{y}$ is the COM in the Y-direction and $m$ is the window size selected to calculate the central intensity $I_{c}$. The value for the window size used to calculate $I_{c}$ was twenty-one. This value was selected as values lower gave erratic changes in central intensity for different frames, and values above reduced the overall central intensity value. 
Increasing the size between these two limits allows the algorithm to have a higher probability of the directional emittance phenomena falling within the threshold window. Increasing these limits too much however raise the chances of incorrect melt pool measurements caused by noise. Using a threshold window has vastly reduced the size of the signal that is to be further processed. This results in parts of the signal with high noise not interfering with edge detections. The process of applying a threshold to the smoothed signal for feature extraction is displayed in Figure 43.

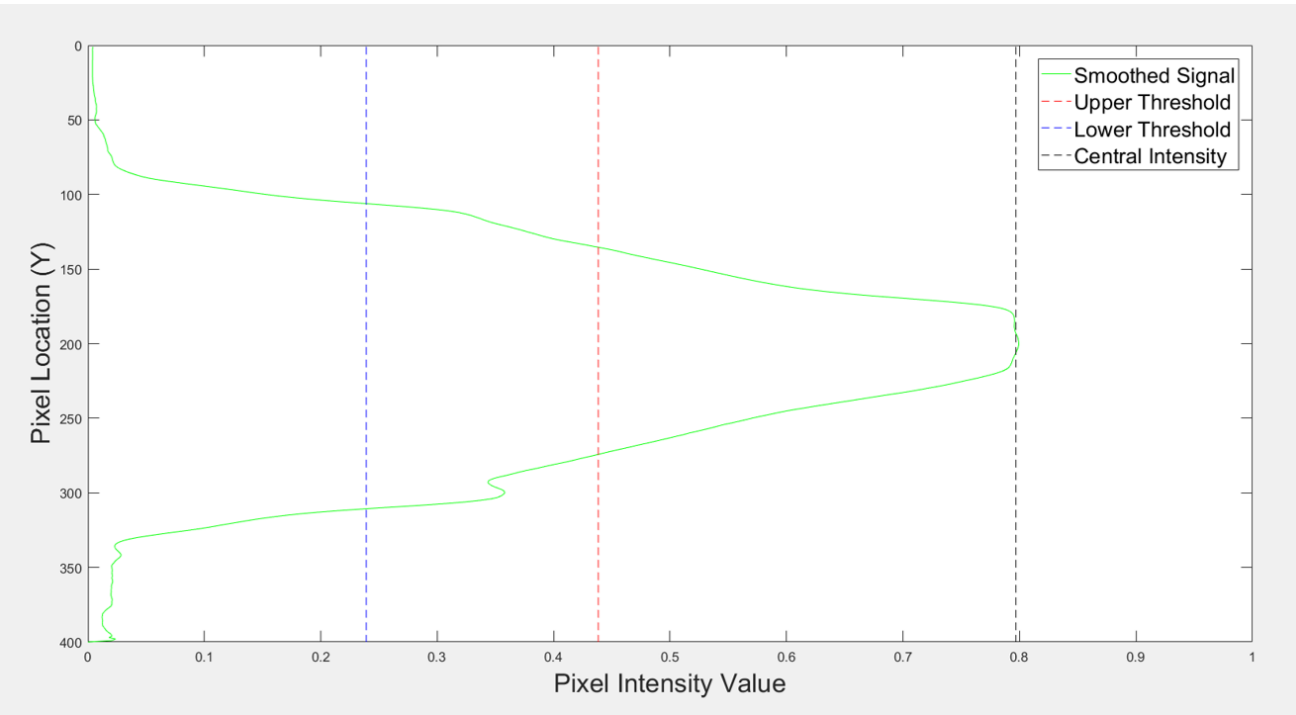

Figure 43: A figure displaying the smoothed signal with the calculated central intensity, lower threshold and upper threshold superimposed.

\subsubsection{Differentiating and extracting}

Once the average image has gone through the relevant averaging signal extraction, smoothing and threshold calculations, it is differentiated to further highlight the directional emittance phenomena. Taking a differential of the windowed signal highlights the feature of interest and makes it more prominent for location calculation. The feature in the differentiated signal is the location of the highest positive gradient (or negative when working in the negative Y-direction). This occurs when the radiation from the substrate changes the normal decaying fashion of the signal.

Once the signal is differentiated, the feature that resembles the edge of the melt pool (caused by 
directional emittance) corresponds to the maximum/minimum value on the differentiated signal when operating in the positive/negative Y-directions respectively. Figure 44 displays the extracted signals for both the north and south locations on the left, with the corresponding differentials on the right. The calculated north and south locations are displayed with the dashed lines for ease of viewing. The calculated north and south locations of the melt pool are plotted on the original image with its corresponding smoothed signal presented in Figure 45. The north and south location values can be used to extract the melt pool width.
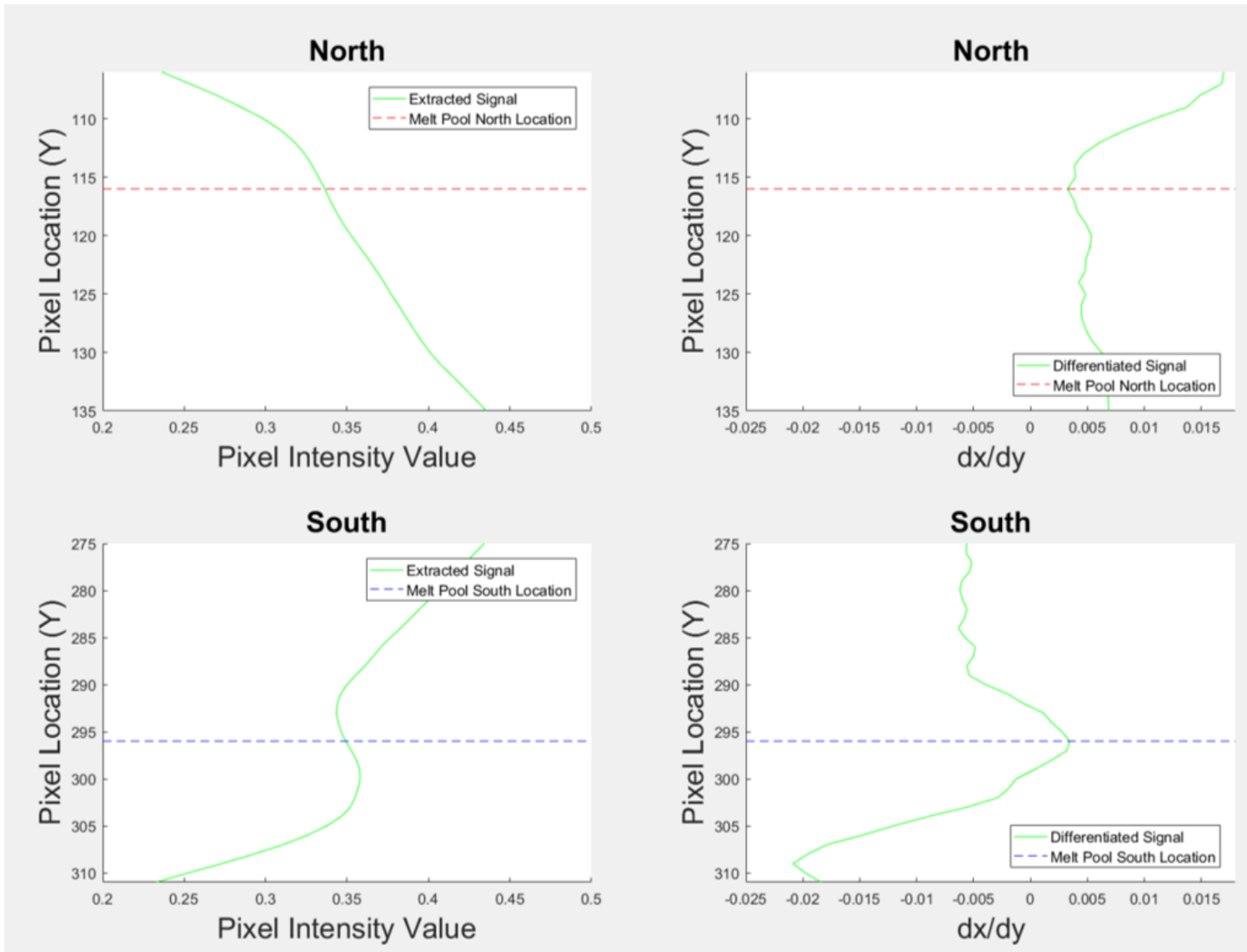

Figure 44: A figure displaying the differentiated signal sections with calculated north and south locations. 

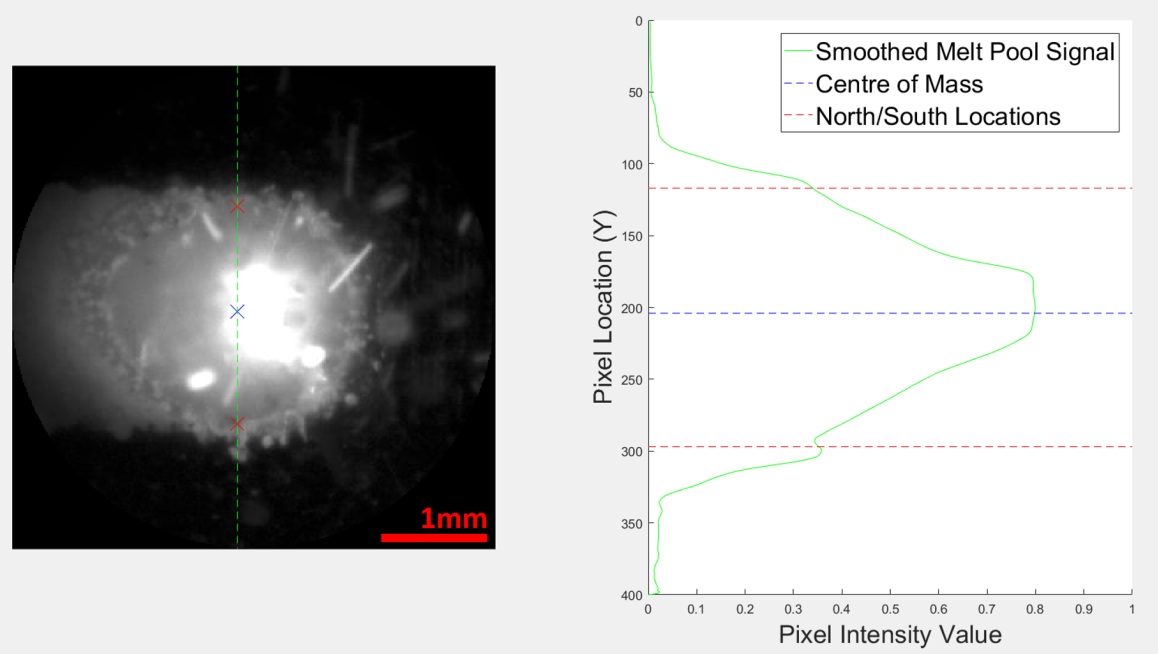

Figure 45: Left: The calculated north and south locations superimposed on the original melt pool image. Right: The corresponding smoothed signal used to extrapolate north and south location values.

It can be seen from this image that the edge of the melt pool is at the same position as the changes in gradient that were highlighted in the raw, averaged and smoothed signal (Figure 42). It has been identified that the kink in the smoothed signal directly relates to both the directional emittance feature and the melt pool edge.

The equations for extracting the north and south locations of the melt pool using the upper threshold, lower threshold and smoothed signal are written in Equations 15 and 16.

$$
\begin{aligned}
& \min _{\substack{y<y<C_{y} \\
L<T_{y}<U}}\left(\frac{\Delta T_{y}}{\Delta y}\right)=z \quad y_{\text {North }} \quad \text { such that } \quad \frac{\Delta T_{y_{\text {North }}}}{\Delta y}=z \\
& \max _{\substack{y: y>C_{y} \\
L<T_{y}<U}}\left(\frac{\Delta T_{y}}{\Delta y}\right)=q \quad y_{\text {South }} \quad \text { such that } \quad \frac{\Delta T_{y_{\text {South }}}}{\Delta y}=q
\end{aligned}
$$




\subsection{System Validation}

This research has witnessed the optimisation of machine vision techniques and the development of a new image processing algorithm to extrapolate melt pool dimensions with improved theoretical understanding. These two techniques have been combined together to produce a system that aims to detect melt pool dimensions at a range of different laser power settings but has yet to undergo validation testing to determine the system's capabilities.

Validating new melt pool dimensioning algorithms is a difficult task to perform accurately and is not something that has been discussed in the field. Many melt pool dimensions have been quoted in previous literature, but there is no way of being able to compare the computed melt pool size with the actual physical molten material. By the time calculations are performed on the image, the melt pool itself has solidified and changed size. This research aims to validate melt pool measurements performed by the newly developed algorithm by comparing results to hand calculations, Canny edge results and track width measurements. The accuracy of the system should be calculated by comparing the image processing calculations with the actual size of the molten melt pool, but harsh environmental conditions including high temperature and stray powder particles prevent these measurements.

\subsubsection{Experimental Aim}

The aim of this experimental section is to distinguish how well the newly designed system can produce melt pool dimensions as compared with the conventional Canny edge detection technique. This is achieved by using the DED system to produce single line cladding tracks and recording the process to produce a database of melt pool videos. These videos will then be subjected to both image processing techniques and compared with each other.

\subsubsection{Experimental Methodology}

\subsubsection{Machine Parameters and Recording Techniques}

This experiment was carried out using the set-up discussed previously in Section 3.4, with the machine depositing single line tracks with lengths of $150 \mathrm{~mm}$. The direction of travel was fixed, the carrier gas 
was set to $3.5 \mathrm{~L} / \mathrm{min}$, the shielding gas was set to $6 \mathrm{~L} / \mathrm{min}$, the path velocity was $11.25 \mathrm{~mm} / \mathrm{s}$, the laser spot size was $1.5 \mathrm{~mm}$ and the PMFR was set to $4.8 \mathrm{~g} / \mathrm{min}$. The powder used for all deposition was EN25. All these settings remained constant throughout the experiment. Using the IDS-imaging software, videos were recorded for multiple laser power settings and exposure times. The laser power setting was iterated from $600 \mathrm{~W}$ to $1200 \mathrm{~W}$ with a total of twenty-one increments $(30 \mathrm{~W})$. To compare the two techniques together, two separate cladding deposition tracks were required for each laser power setting. One was taken using a fixed exposure time for all laser power settings and the other was taken using the adaptive exposure times calculated using Equation 1. The fixed exposure time selected used Equation 1 to find the best exposure time setting for the laser power setting of $600 \mathrm{~W}$. This exposure time was then kept constant throughout the experiment.

The recorded videos for this experiment were then saved to a hard disk so that post event image processing could be carried out. The videos were loaded into a MATLAB environment and were subject to either the Canny edge detection algorithm or the directional emittance algorithm for direct comparison.

\subsubsection{Hand Calculations}

Hand calculations were also performed on the same videos by selecting multiple frames and measuring the melt pool width by eye. A small program was written in MATLAB that allowed the user to extract random frames, and alter the position of dashed lines to aid in the extraction of melt pool width measurements. The dashed lines were moved until they were directly over the directional emittance features. The program then calculated the melt pool width using the dashed line locations. For each video, twenty hand calculations were performed before average values were calculated.

\subsubsection{Track Width Measurements}

Track measurements were also performed on the deposited tracks, to correlate algorithm measurements to actual track widths. Deposited tracks were subject to optical microscope imagery using a VHX-700F digital microscope. Multiple width measurements of deposited tracks (laser power settings of 600, 800, 1000 and $1200 \mathrm{~W}$ ) were performed using the in-built measuring tools in the VHF software. 


\subsubsection{Results}

Conducting this experiment gave rise to a substantial amount of data. For each of the forty-two tracks deposited, around 800 frames were recorded. The data for the tracks can be represented by either comparing individual frames, or average values across the deposited track. The results of this section will firstly compare calculated melt pool widths of individual frames for different laser power settings for both image processing techniques. These frame values will then be used to calculate the average melt pool width across the cladding track to allow for the comparison between both image processing algorithms, hand calculations and track measurements for different laser power settings. Finally, this section will detail image processing calculations on individual frames, and how incorrect measurements can occur.

\subsubsection{Melt Pool Dimensioning - Frame Comparison}

The first analysis conducted was a direct comparison of melt pool signals calculated from the original Canny edge technique and the new directional emittance technique. To compare these signals directly to one another, snippets were extracted from the original full melt pool dimensioning data. Taking snippets from the recordings allowed for an easier comparison, due to all melt pool signals starting and stopping at different times in different videos. Although all tracks were deposited with the same length, the amount of frames that calculated melt pool dimensions varied slightly between each recording. The amount of frames that gave melt pool measurements were calculated for each video and an average of these values were taken for all forty-two recordings. The length of the snippets that were extracted from the original video were half of the average length calculated across all recordings. The deposition start point was determined when the first melt pool measurement was recorded for each individual track. The starting point for snippet extraction began after $25 \%$ of the average track length had passed and finished before the last $25 \%$ of the average track length. This resulted in signals that were not clouded by the unstable nature of the melt pool in the acceleration/stabilisation and deceleration/destabilisation stages of deposition. Figure 46 shows an original melt pool signal, with guide lines to display the extraction process. The frame numbers on the extracted signal are not 
carried over from the original signal and reset to one.
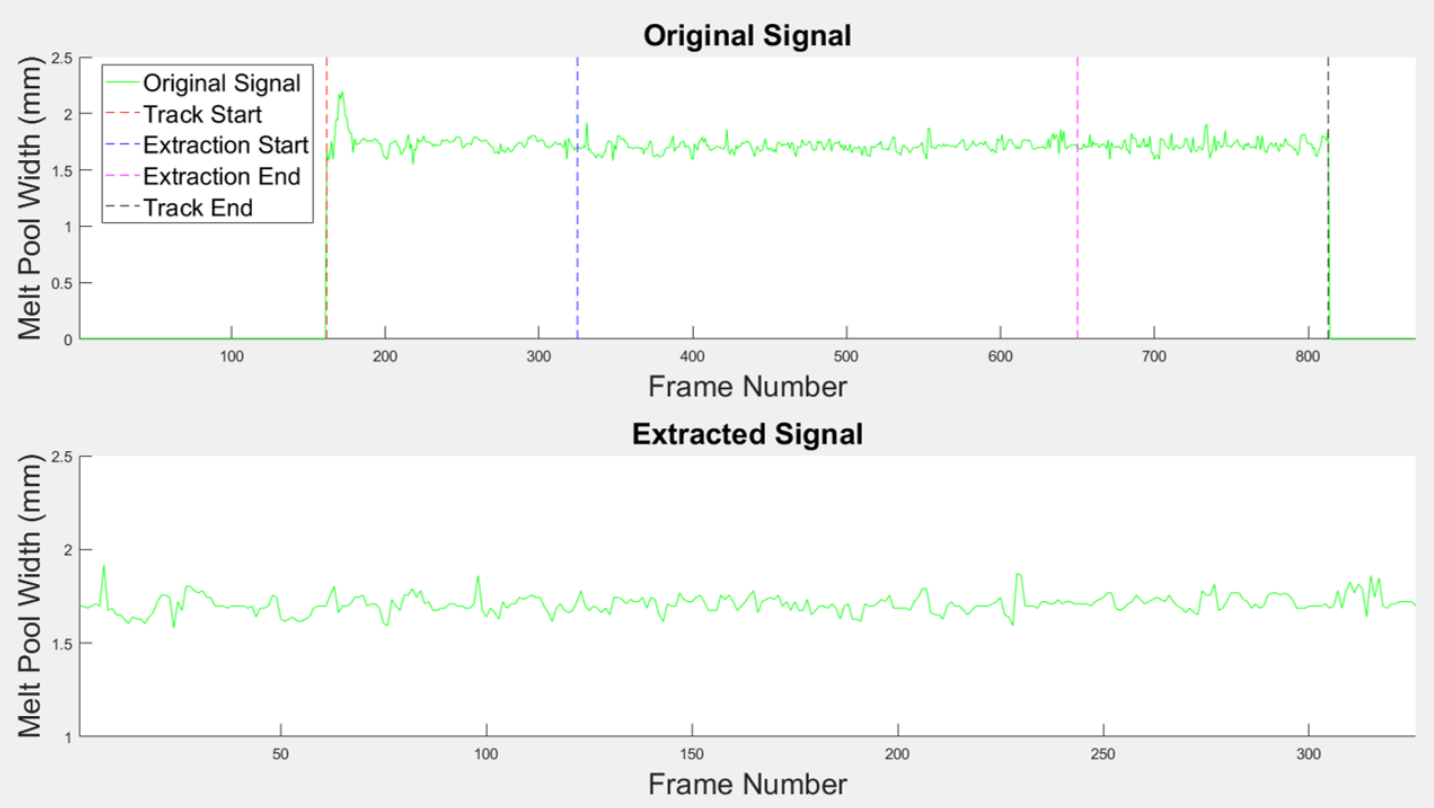

Figure 46: Top: An original signal of the melt pool width calculations for a recording of a track deposition. Bottom: The extracted signal used for melt pool dimensioning comparison.

Using this extraction technique, the signals from the forty-two recordings were compared to each another to establish the stability of the readings and the difference between the two techniques. The results from all signals were similar, and the three comparisons displayed in Figure 47 are solid representations of all signal comparisons.

The melt pool width extracted from videos using both techniques showed similar readings for both the Canny edge and directional emittance techniques. For lower laser power settings, the Canny edge technique gave larger melt pool width values. The Canny edge technique overestimated the size of the melt pool incorrectly for laser power settings of around $750 \mathrm{~W}$. This was due to the technique measuring radiation from both the melt pool and the surrounding substrate. At this point, the average melt pool width values became very similar, with the Canny edge detection technique producing very similar results to the newly designed directional emittance technique.

The extracted signals shown in Figure 47 show that the Canny edge technique produces a much 
$600 \mathrm{~W}$
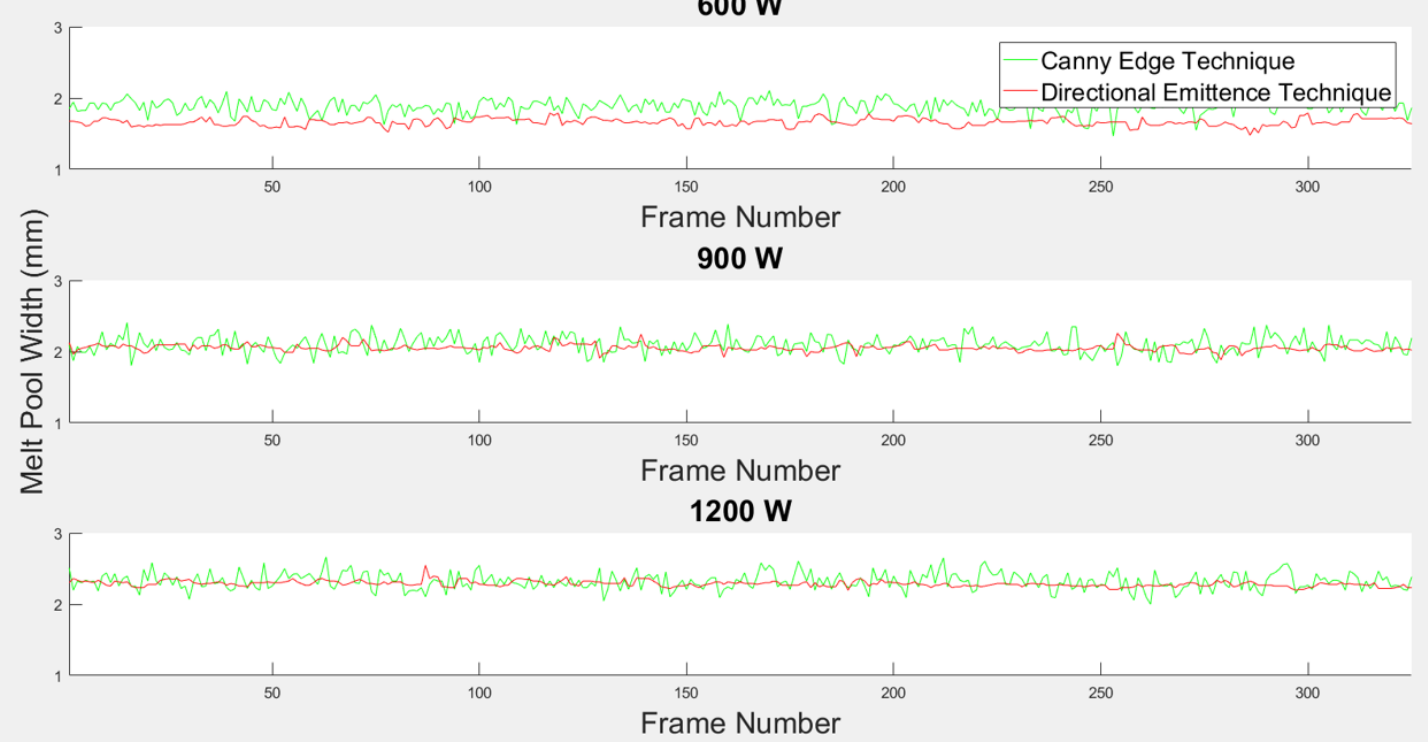

Figure 47: Top: An original signal of the melt pool width calculations for a recording of a track deposition. Bottom: The extracted signal used for melt pool dimensioning comparison.

more erratic signal for the melt pool width. The Canny edge technique shows a greater difference between its maximum and minimum melt pool width values and was greatly influenced by un-melted powder particles and flares. The directional emittance technique displays a much less erratic signal and shows less melt pool width extremes for all laser power settings. The direct comparison between these two image processing techniques for all laser power settings shows that the newly directional emittance algorithm produces less erratic, less extreme and more stable melt pool measurements that are less prone to image noise.

\subsubsection{Melt Pool Dimensioning - Track Comparison}

After comparing individual melt pool track snippets, averages were taken from the snippets to compare both techniques with measured hand calculations. The snippets were used to calculate the average melt pool width by simply adding up all melt pool width values and dividing them by the total number 
of values. Snippets were used so that the acceleration/stabilisation and deceleration/destabilisation sections of the track deposited had no effect on the average end result. The averages were then plotted against each other, to establish how the melt pool width is influenced by the laser power setting. The results of this study are displayed in Figure 48.

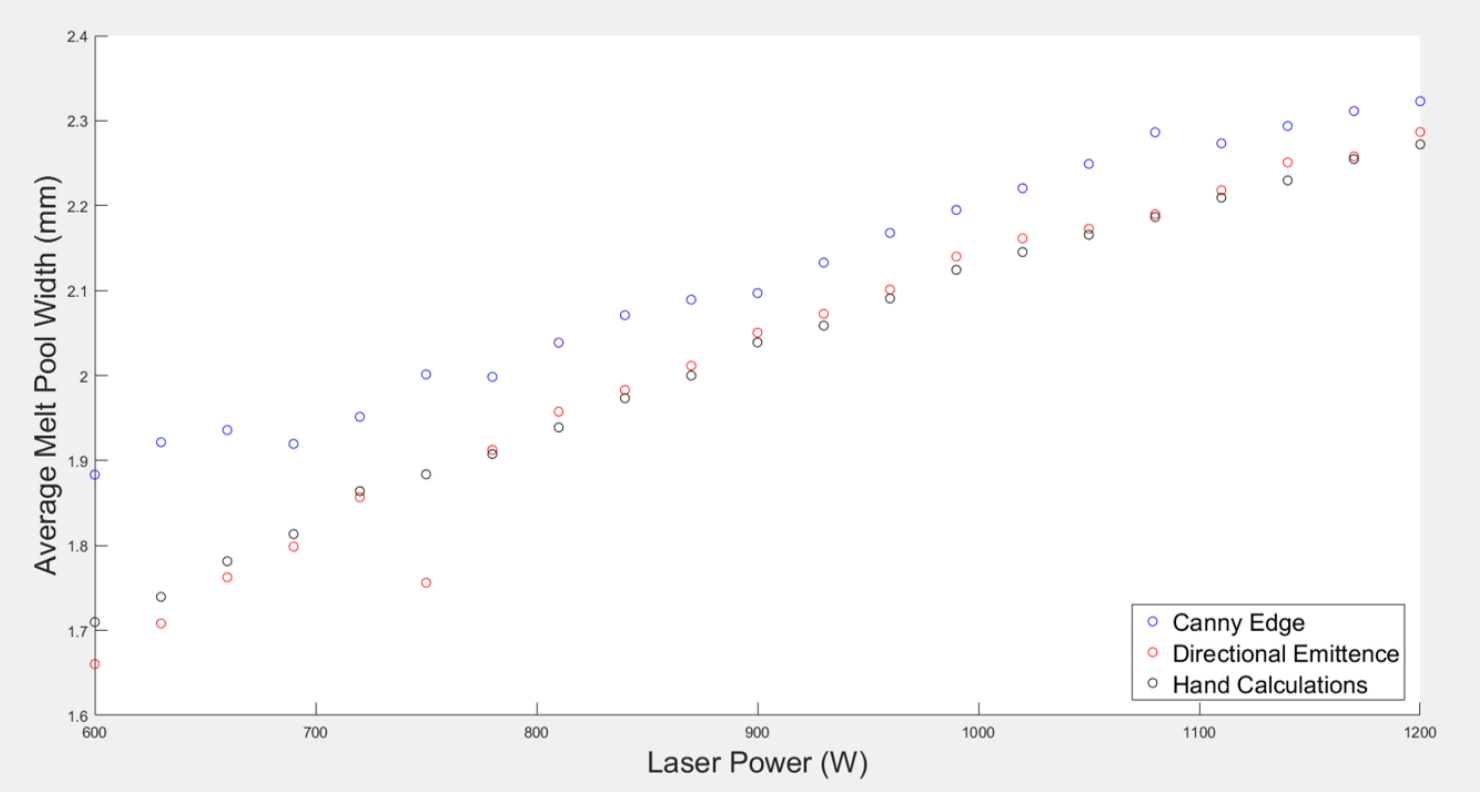

Figure 48: A graph representing the average melt pool width values for a range of different laser power settings.

Figure 48 shows calculated melt pool width averages from the Canny edge, directional emittance and hand calculation techniques. The graph shows that there are large differences between the calculated Canny edge detection values and both the directional emittance and hand calculations for the lower laser power settings. Observing these videos highlighted major issues with using the Canny edge technique to calculate melt pool width values for individual frames. This will be expanded upon in Section 4.3.3.4.

The directional emittance measurements have a very strong relationship with the hand calculations. Both of these techniques display a similar relationship and all data points of the direction emittance measurements (apart from the one anomaly) are closer to the hand calculations than the Canny edge 
measurements. Increasing the laser power does increase the size of the melt pool, but the directional emittance measurements and hand calculations show that this relationship is not a rudimentary linear relationship. The anomaly that is present at a laser power setting of $750 \mathrm{~W}$ for the directional emittance algorithm was removed from the graph. This case is discussed in detail in Section 4.3.3.5. With the anomaly removed, a second order polynomial was fitted to all sets of data to see how well they abide by this trend. The results of this are shown in Figure 49.

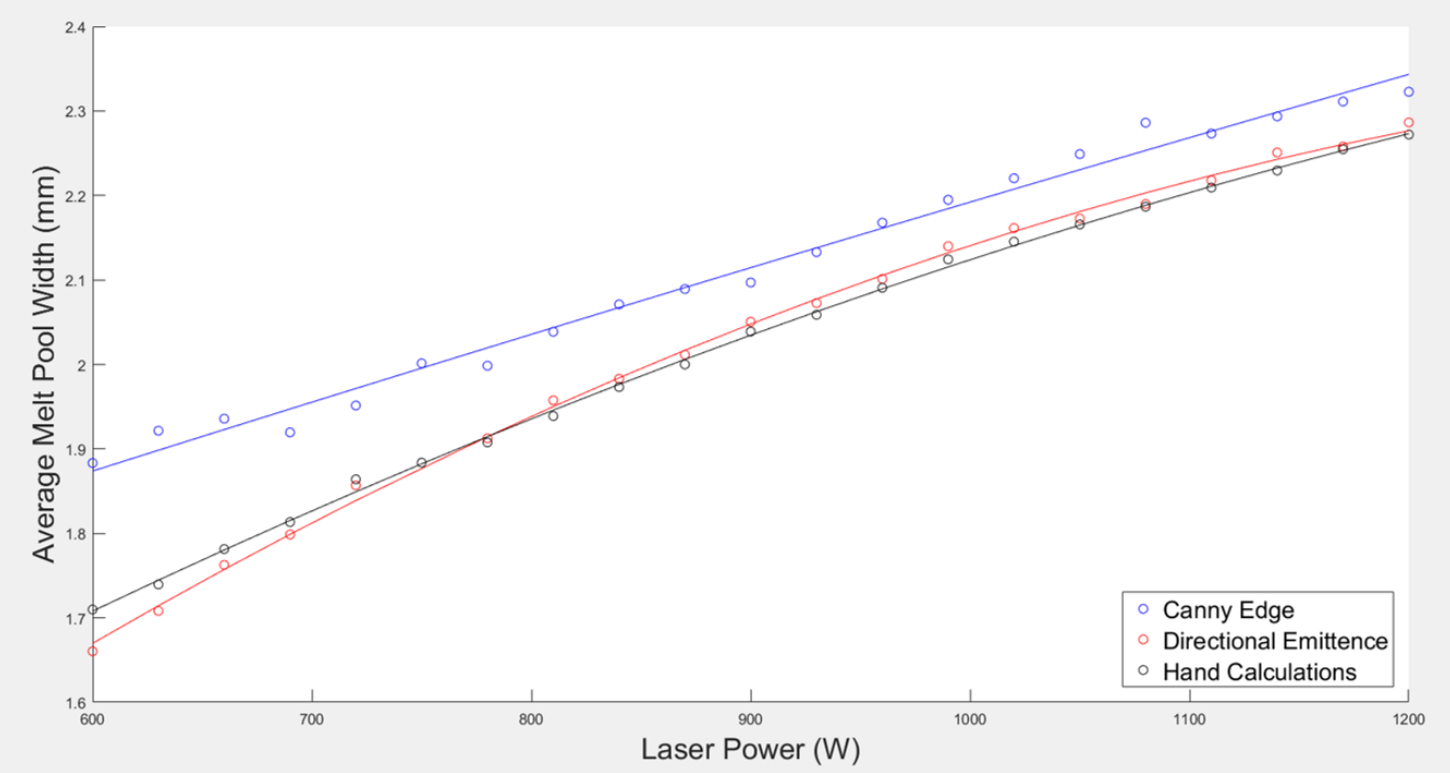

Figure 49: Top: An original signal of the melt pool width calculations for a recording of a track deposition. Bottom: The extracted signal used for melt pool dimensioning comparison.

The Canny edge technique shows more of a linear relationship, and is due to the fact that the algorithm is linked more closely to the intensity of the image, rather than the actual size of the melt pool. The newly designed directional emittance algorithm shows a relationship that is extremely close to the second order polynomial displayed in Figure 49.

The theory behind this polynomial relationship is that increasing the laser power in the DED process will increase the size of the melt pool, but the size of the melt pool is restricted to the amount of powder in the given situation. The melt pool, for this material, begins to establish a stable droplet 
at a laser power setting of around $600 \mathrm{~W}$. This low laser power setting has an abundance of powder particles available to the melt pool, and therefore when the laser power is increased (to around 900W), more of the available powder can be absorbed into the molten melt pool to greatly increase its size. At a laser power setting of around $900 \mathrm{~W}$, the increasing of melt pool width with regards to laser power begins to decrease. This is thought to be because the laser power is no longer the major restriction in the growth of the melt pool, but rather the amount of powder that is being injected. Increasing the laser power beyond this point results in a larger calculated melt pool width, but this could be down to the laser power melting more of the substrate, rather than the injected powder particles. The rate at which the melt pool width grows with increasing laser power settings is reduced when depositing at $900 \mathrm{~W}$ and above. This is true with the specific parameter settings used in this experiment for the material EN25.

To determine the increased accuracy of the newly developed directional emittance image processing algorithm, the hand calculations were deemed to be true values and both the Canny and directional emittance algorithms were directly compared. The directional emittance algorithm calculated melt pool width values within $1 \%$ of the hand calculations. The Canny edge algorithm calculated width values within $4.8 \%$ of the hand calculations.

This graph shows that complex relationships in the DED process can be resolved when more stable and accurate algorithms are used for melt pool measurements. The original Canny edge technique failed to show this second order polynomial relationship. The new directional emittance algorithm displayed a relationship between the width of the melt pool and laser power settings that has not been documented. The new directional emittance algorithm was deemed more accurate than the Canny edge technique when comparing measurements to the hand calculations.

\subsubsection{Correlation With Measured Track Widths}

To further validate the results of the newly developed algorithm computational measurements were directly compared to measured track widths. Figure 50 displays optical microscope images of the track widths with their corresponding measurements. Measurements were taken at different areas along the deposited track and averages were calculated. A total of eighteen measurements for each track were 
used in the calculation of track width averages. Table 4 displays the results.
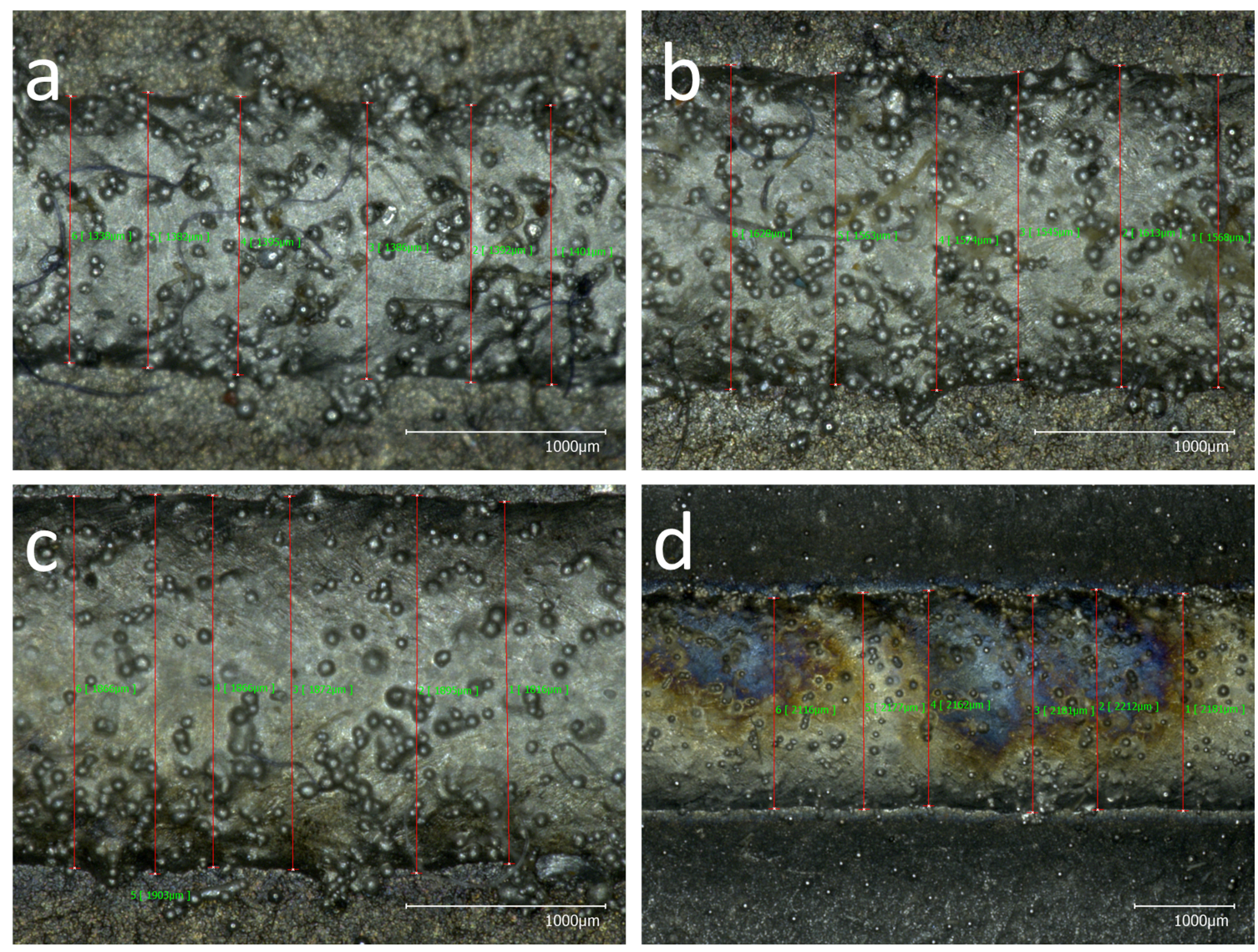

Figure 50: Microscope images with corresponding measurements for different laser power settings. a) $600 \mathrm{~W}$ b) $800 \mathrm{~W}$ c) $1000 \mathrm{~W}$ d) $1200 \mathrm{~W}$

Table 4: Average measured track widths

\begin{tabular}{cc} 
Laser Power Setting $(\mathrm{W})$ & Average Melt pool Width $(\mathrm{mm})$ \\
\hline 600 & 1.402 \\
800 & 1.689 \\
1000 & 1.901 \\
1200 & 2.178
\end{tabular}

To directly compare the results of the track measurements with the different algorithms and hand 
calculations, all data was plotted on the same graph. These results are displayed in Figure 51.

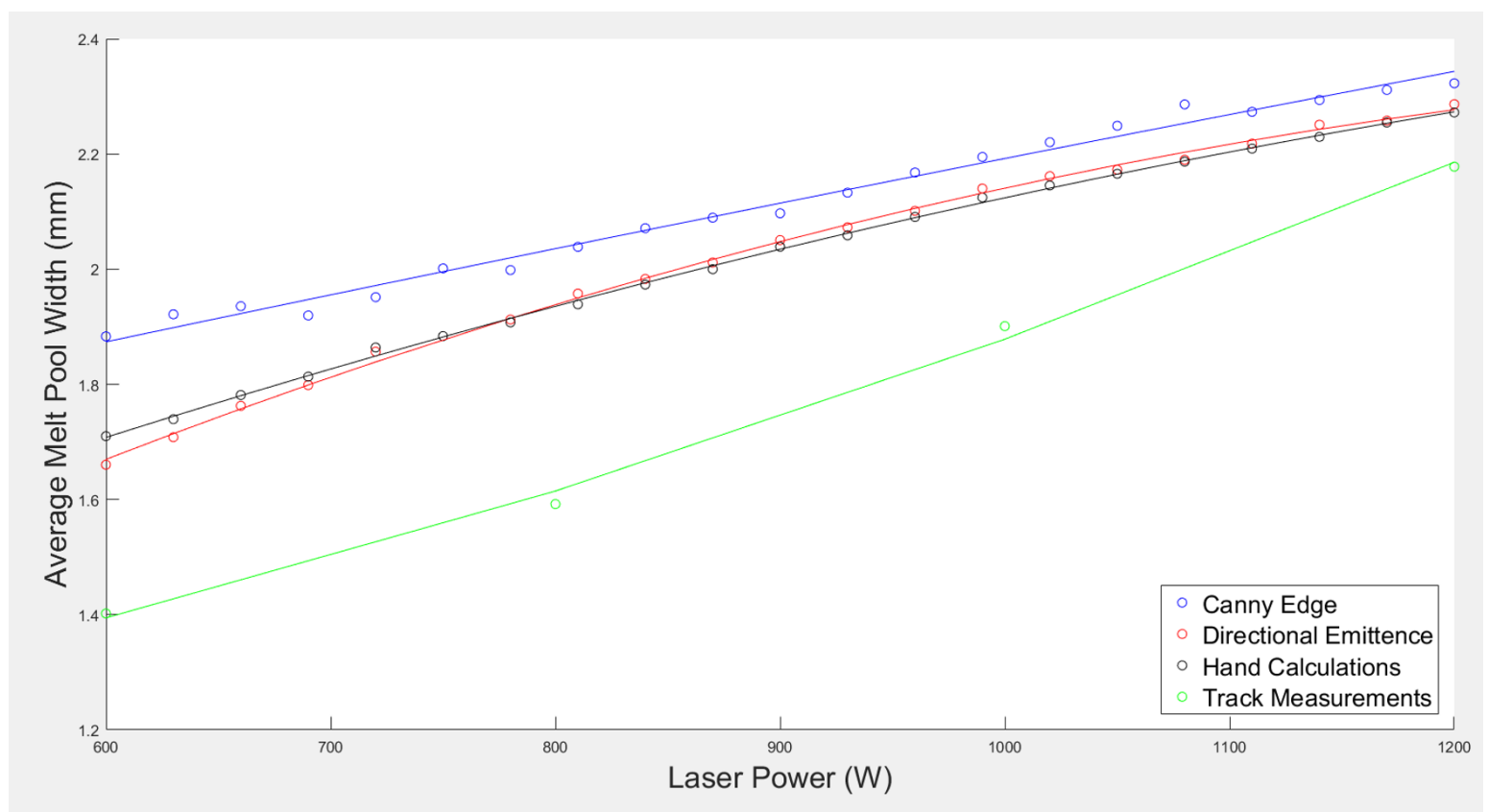

Figure 51: Microscope images with corresponding measurements for different laser power settings. a) $600 \mathrm{~W} \mathrm{b)} 800 \mathrm{~W}$ c) $1000 \mathrm{~W}$ d) $1200 \mathrm{~W}$

Figure 51 clearly shows that track width measurements are lower than melt pool measurements extracted using both image processing algorithms and hand calculations. This is likely due to the shrinkage that occurs during the solidification stage. Shrinkage occurs due to overall reduction in temperature and contraction during phase transition. Due to the large differences in melt pool and track width measurements, it is difficult to use track measurements to establish the accuracy of the image processing techniques. The newly designed image processing technique measurements are closer to that of the track measurements, but this does not necessarily mean the algorithm is more accurate. In one case we are measuring molten metal and in the other we are measuring solid metal at room temperature. The data shown here also indicates that the track measurements show a polynomial relationship but the relationship is unlike that experienced in the newly developed algorithm and hand calculations. Increasing the laser power at higher laser power settings has more of an effect on 
measured track width than increasing laser power at lower laser power settings.

The polynomial relationship of track measurements is thought to occur due to a phenomena that has not been reported in literature. This research labels this phenomena as 'slumping' and it occurs as the melt pool solidifies to form the deposited track. Slumping occurs during the solidification process due to the melt pool being in its liquid state for a period of time. Throughout this time, the forces of gravity act on the melt pool causing it to spread laterally before phase changes stop this movement and solidify the track. At higher laser power settings, the amount of time that the melt pool will be in its liquid state (when slumping can occur) is increased.

This relationship between the track and melt pool width is one that is likely to be complex. Differences in these trends could be a result of both shrinkage and slumping. Depositing at higher laser power setting will increase the amount of time it takes for the melt pool to solidify and thus increase the amount of slumping that can occur. This provides a explanation for the relationship between melt pool and track width calculations, but is something that will require more study. Due to the complex relationship between measured track and melt pool widths, the determination of image processing algorithm accuracy by correlating calculations with measured track widths was deemed unreliable.

\subsubsection{Detailed Canny Edge Frame Analysis}

As previously discussed, establishing how well an algorithm calculates the physical width of the melt pool is difficult. Measurements using emissivity values (including the Canny edge detection technique) have been documented to give readings with low accuracy [39, 40, 47], and physically measuring the melt pool width is impractical. For this reason, this section of work will aim to determine how well the Canny edge algorithm calculates the width of the melt pool in a given image. Slight variances between the physical melt pool and recorded digital images may be present, but with the current state of the art this is the most accurate representation of the melt pool.

The conventional method of calculating melt pool width uses an emissivity based threshold [10, 24, 27, 30-32, 34, 43-46, 60, 86, 87], most commonly followed by a Canny edge detection algorithm $[10,27,31,45]$. Raw melt pool images are subject to a binary threshold with the threshold value 
defined by the user. This threshold value is calculated by correlating a pixel intensity value with the materials melting temperature using an emissivity value. The binary image is then subjected to erosion and dilation before the Canny edge image processing technique is used to identify the melt pool edges. The Canny edge image processing technique is a five step technique that uses a Gaussian filter to smooth the image, finds intensity gradients, applies a non-maximum suppression, applies a double threshold, and then tracks edges using hysteresis to suppress weak edges and highlight strong edges [88]. Details of the Canny edge detection technique are not documented here due to the extensive nature of the technique. The full technique can be found in the following reference [88].

Trying to use the Canny edge technique to calculate the width of the melt pool is incredibly difficult. Due to previous literature highlighting the issues with using emissivity values, and the complexities with achieving these emissivity values, no emissivity values were used to calibrate the threshold value. Instead of using an emissivity value to calculate the binarisation threshold, the binarisation threshold was changed by the user, until measurements calculated by the Canny edge image processing technique were as close to the recorded images of the melt pool as possible. Using this technique to attempt to calibrate the Canny edge image processing technique uncovered fundamental flaws in the technique which are to be discussed.

The original Canny edge detection technique relies on emissivity values, but it was discovered that it also greatly relies on the melt pool being symmetrical in shape and the camera being perfectly aligned to capture a true birds-eye view of the melt pool. This ideal scenario is incredibly difficult (if not impossible) to achieve and the experimental set-up showed that even minute differences in the melt pools relative angle can cause large differences in melt pool imagery. For instance, melt pools shapes can change due to the surface angles of the underlying substrate. In addition the laser beam needs to be exactly perpendicular to the substrates surface and powder must enter the melt pool in a symmetrical manner. Even when care is taken to ensure as much symmetry as possible, particles of different weights and sizes are injected from different angles with random distribution patterns. The substrate being deposited on is not perfectly flat, and even if it was, it will often begin to warp/distort throughout deposition processes. Even if the substrate does not warp, depositing multiple layers on top of one another causes the melt pool to be deposited on uneven surfaces. When a melt pool is deposited 
on top of another track, this track will not be flat and will cause the melt pool to be unsymmetrical. Some deposition techniques introduce a brick-like layering systems to help with build stability, which again causes the melt pool to be unsymmetrical. Overhang structures and depositing material at angles will again cause unsymmetrical melt pool geometry. Trying to align all these factors together in an industrial environment is neither practical nor affordable. A robust melt pool dimensioning algorithm should be able to cope with imperfect environments if it is to be used in industrial applications.

Figure 52 is an original melt pool image with it's corresponding binary image after it has been subjected to the binerisation, erosion and dilation processes. The image that is seen here is typical of the melt pool in the DED process with a laser power setting of $600 \mathrm{~W}$. This image shows the calculated melt pool width using the Canny edge detection technique.

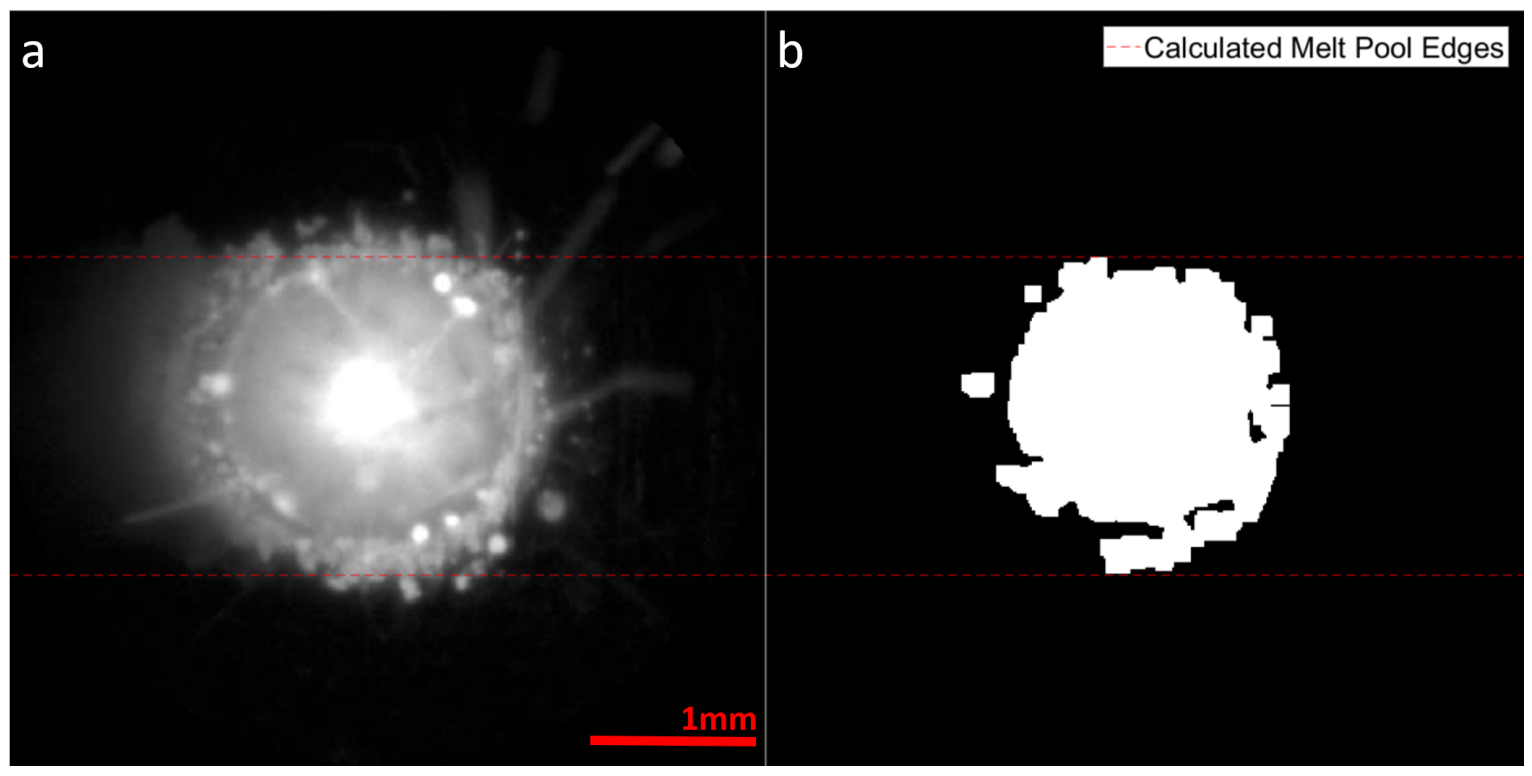

Figure 52: a: An original melt pool image with calculated melt pool edges using the Canny edge detection technique. b: The melt pool binary image after it has undergone the binarisation, erosion and dilation processes.

The resultant melt pool width calculated in this image clearly does not reside around the edge of the melt pool in the picture. The calculation of the melt pool's north location in this image appears to be correct for this given frame, however the south location is completely off. The calculated south 
measurement resides in the region of illuminated substrate. This single frame exposes issues with the Canny edge detection technique that has not been seen before due to the quality of images obtained by previous state of the art systems. Taking a single threshold of an image is not enough in calculating the melt pool width, even if one can calculate an accurate emissivity value. The reason for this is because the melt pool in this image does not show a symmetrical distribution of radiation when moving away from the melt pool centre. Changes in surface angles relative to the cameras FOV cause certain regions of the image to show different intensities for what may be the same temperature. If directional emittance had no effect on radiation being received by the CMOS centre, then the north location and south location would occur at the same threshold level. Real grey bodies do not emit radiation equally in all directions, meaning that the north and south locations will often occur at different pixel intensity levels in the image. Figure 52 shows one correct and one incorrect melt pool edge measurement displaying that the interface between liquid metal and surrounding atmosphere/substrate cannot simply be explained by a singular emissivity or threshold value.

Figure 53 shows another image taken from the exact same video recording, using the same binary threshold. This image backs up this theoretical explanation by showing two incorrect measurements of the melt pool edges. In this image, one of the edges is deemed to be inside the molten metal, whilst the other is deemed to be in the illuminated surrounding substrate. Both calculations are extracted from the same binary threshold image. Figure 54 shows the melt pools north and south locations calculated using the directional emittance technique for the same frames in Figures 52 and 53. 


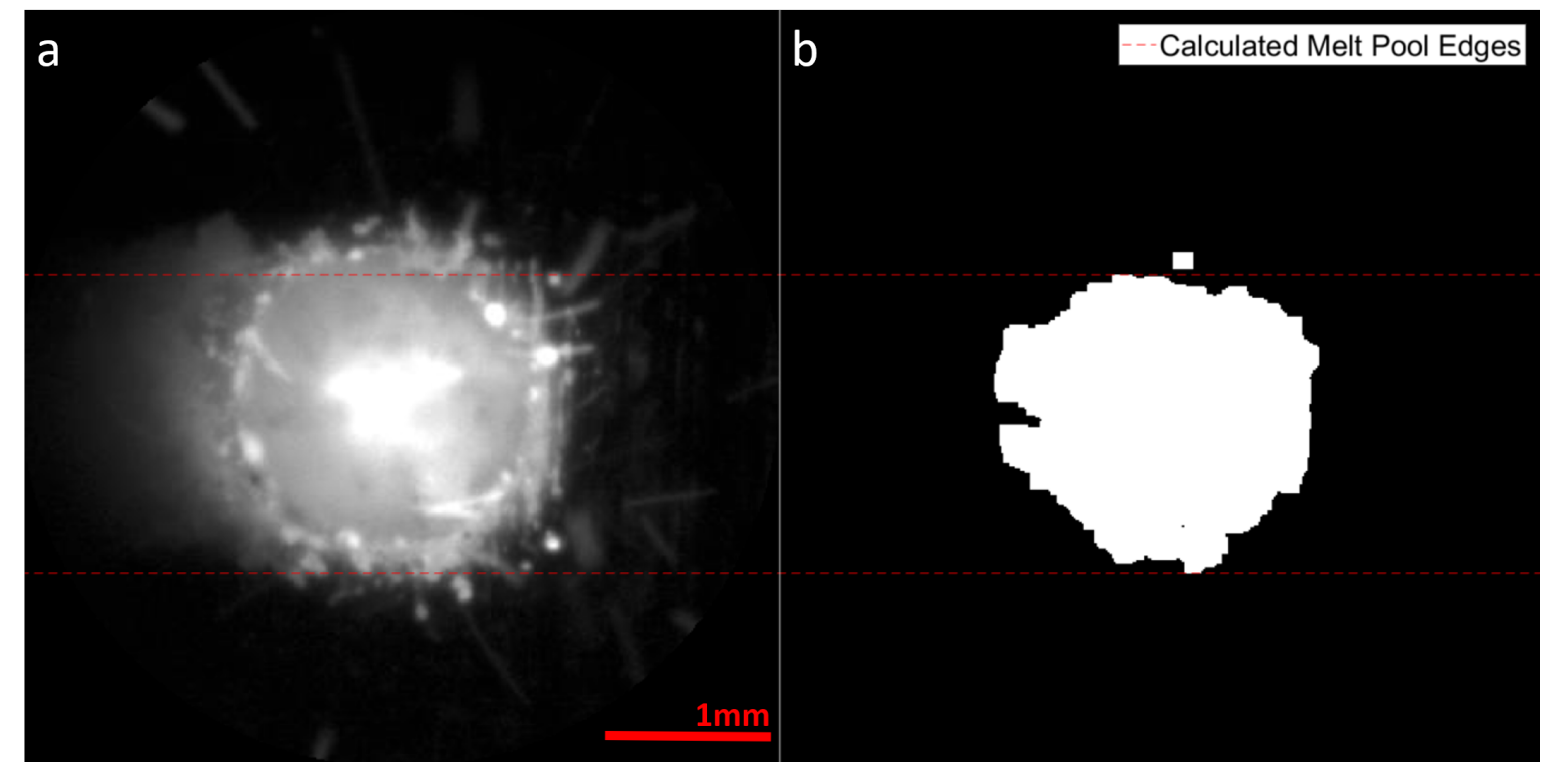

Figure 53: An image showing two incorrect melt pool edge locations using the Canny edge technique. a: Original Image. b: Binary Image.

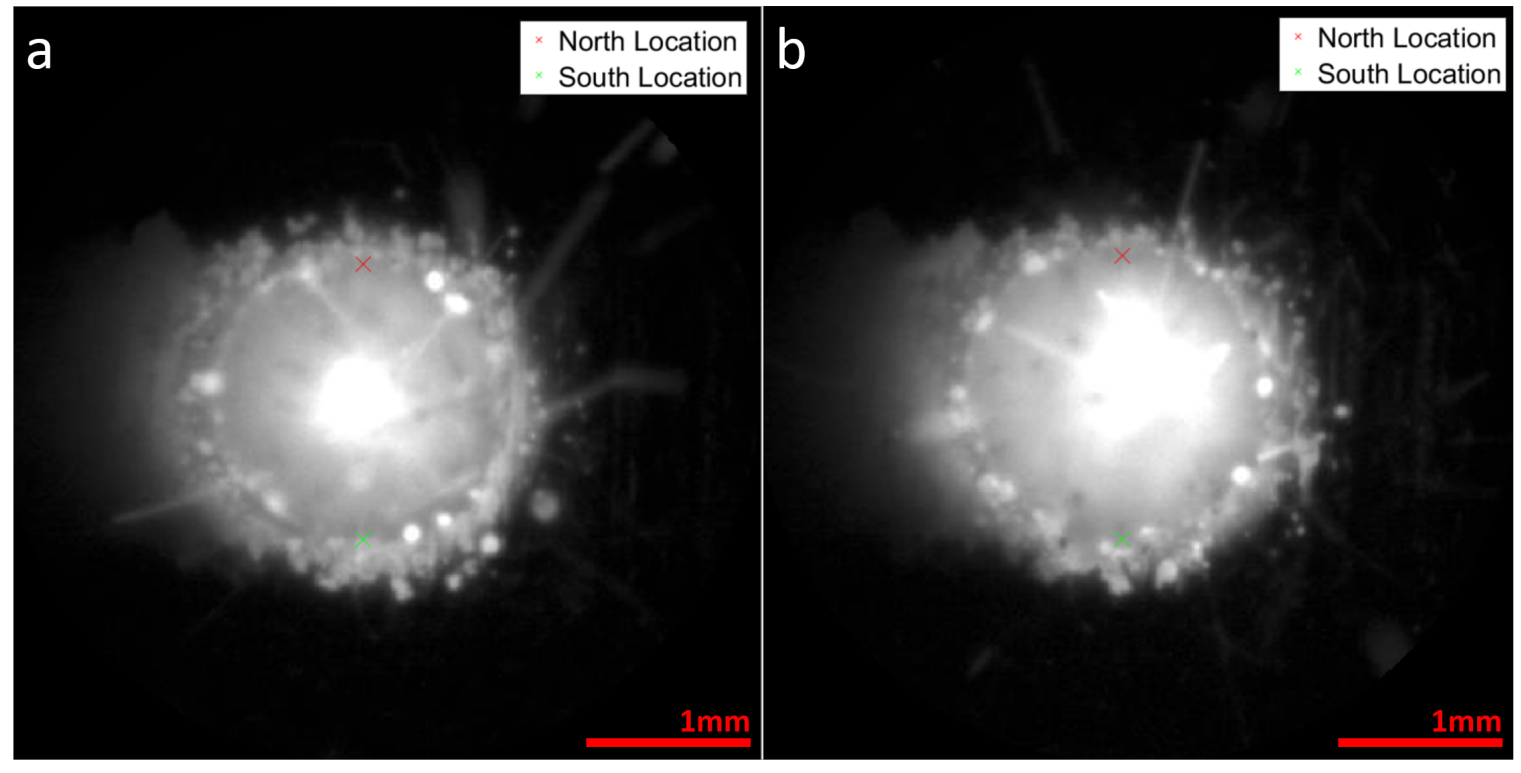

Figure 54: An image showing the north and south locations calculated using the directional emittance technique for frames in Figures 52 (a) and 53 (b). 


\subsubsection{Detailed Directional Emittance Frame Analysis}

All videos that were processed using the directional emittance image processing technique were viewed to monitor consistency in measurements. The new directional emittance image processing technique not only showed a more stable response when comparing melt pool width signals over frames numbers, but also showed good accuracy when individual measurements were compared to hand calculations. Figure 55 shows examples of frames that show accurately measured north and south locations.

From the frames presented, and observing the image processing technique as it was performing on each frame, it was clear that the new image processing technique had few failed frames. The percentage error between the directional emittance and hand calculations also compliments this technique.

Although the image processing technique was deemed to be accurate for most videos, one video in particular provided difficulties. The video recording the track deposition on a laser power of $750 \mathrm{~W}$ produced an anomaly (Figure 48). This recording showed an average melt pool width for the track much lower than expected. When observations of the image processing technique being executed on the video were made, the north location appeared to fluctuate throughout the video. Some of the readings showed the north location to be inside the melt pool itself.

This video recording was analysed in detail to establish the causation of these false melt pool regions. It was found that for this particular video, the directional emittance phenomena was not as prominent as it was in the other recordings. The reduced presence of the directional emittance phenomena in these frames caused the algorithm to locate the north location of the melt pool inside the molten metal region where noise (from stray particles, un-melted powder particles and dark regions) caused rapid changes in intensity levels. The noise in these frames showed greater changes than the edge of the melt pool, leading to false readings. Figure 56 shows an example of this, with the corresponding signal. 


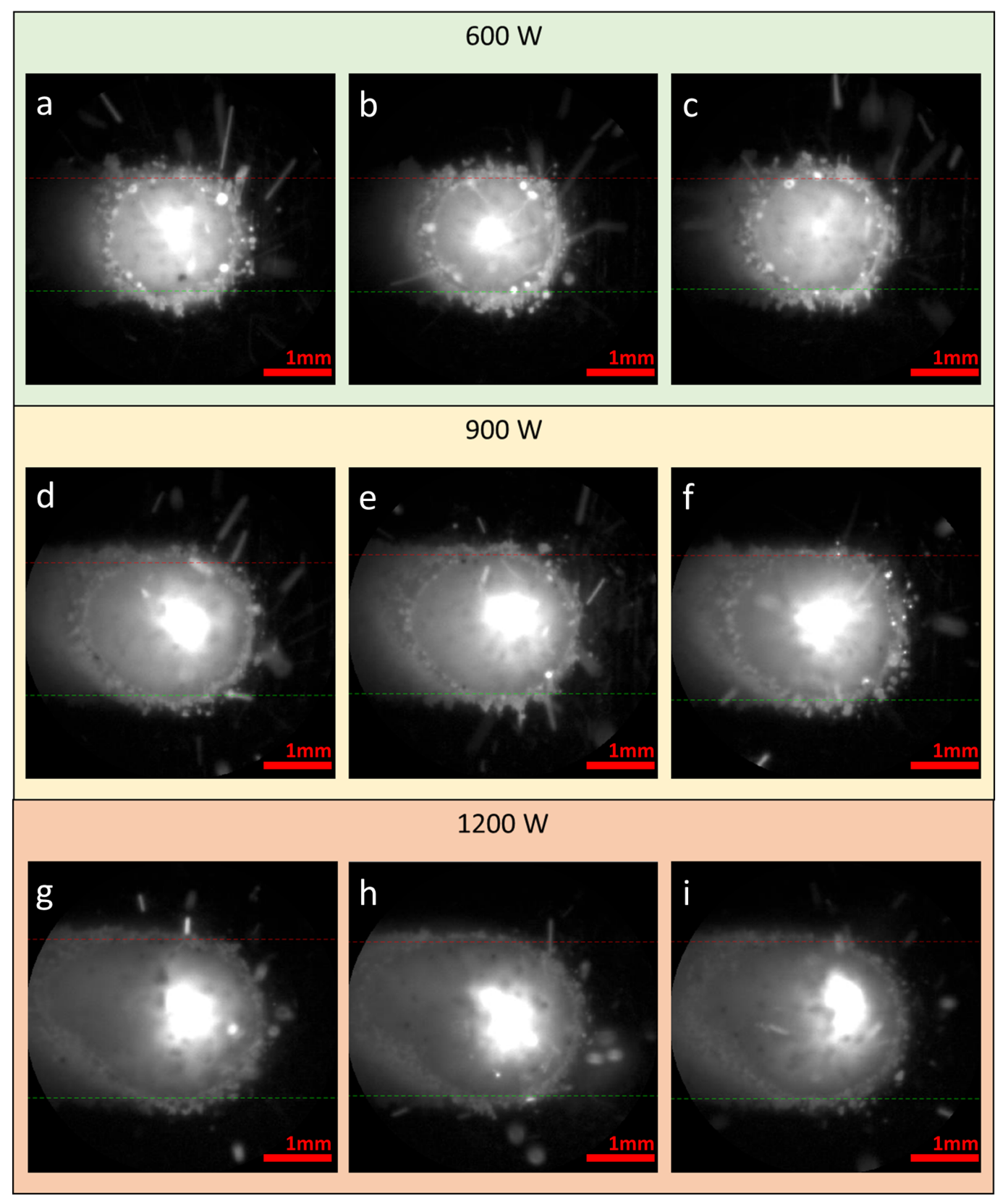

Figure 55: Still frames taken from video recording tracks deposited at 600, 900 and 1200W. Red lines indicate the calculated north location, green lines indicate the calculated south location. 

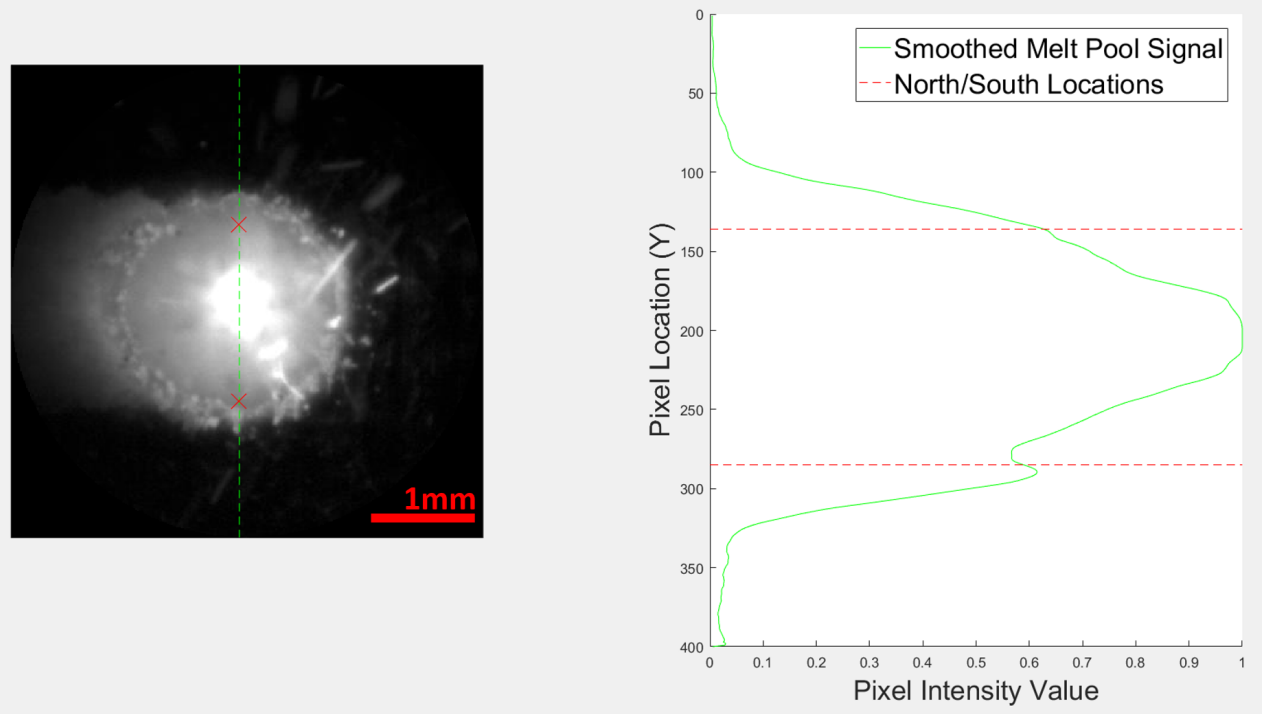

Figure 56: A still frame taken from a video recording with its corresponding smooth signal.

Figure 57 shows a frame with reduced prominence of the directional emittance feature and a lack of a clear melt pool edge. It can also be seen that the corresponding smoothed signal does not have a change in gradient located at the melt pool edge that was witnessed in previous signal extractions (Figure 42). This could be a result of the CMOS sensor not being able to pick up the edge due to incorrect exposure settings and poor focusing. Poor focusing could be a results of the systems small DOF. However, due to the image being in focus, DOF issues are not likely to be the causation.

A more likely explanation is that the actual melt pool is not forming an edge due to unbalanced physical forces. Warped substrate, thermal gradients and uneven powder delivery could all contribute to producing a melt pool of this nature. This lack of melt pool edge formation has resulted in the signal not displaying a kink corresponding to a directional emittance feature. 

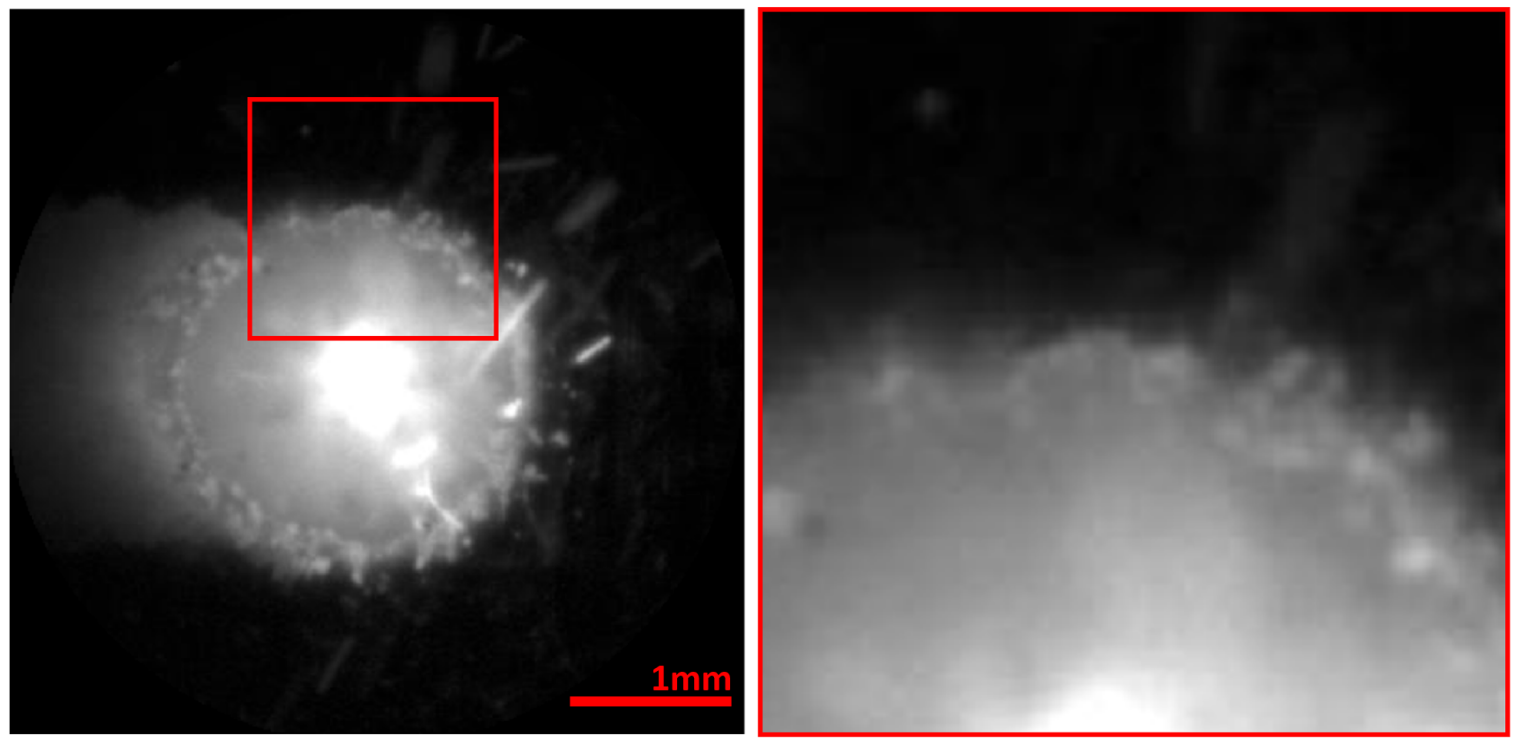

Figure 57: A detailed image of the melt pool displaying the smooth gradient at the edge of the melt pool that the directional emittance image processing technique could not resolve.

\subsection{Discussion}

Research from this chapter aimed to analyse the accuracy of the existing Canny edge melt pool monitoring technique. The research also aimed to develop a new melt pool dimensioning algorithm that was able to determine the width of the melt pool without the need for material dependant emissivity values. Extracting melt pool dimensions without emissivity values will allow users to develop more versatile melt pool monitoring systems that can perform material independent melt pool width measurements. This will allow for a greater uptake of melt pool monitoring techniques in industrial applications. Melt pool monitoring systems with higher accuracy will likely improve process understanding, control system effectiveness and deposition quality.

After the new directional emittance algorithm was developed, both techniques were subject to multiple forms of analysis to determine accuracy and reliability. Melt pool measurements across different laser power settings for both algorithms were directly compared to hand calculations and solidified track width measurements. A detailed analysis was also performed on both techniques by 
extracting individual frames and superimposing measurements onto them. Results showed that the new directional emittance algorithm was more accurate than the conventional Canny edge detection technique. It was discovered that due to the directional emittance phenomena, one cannot simply assume that the melt pool edge emits radiation at the same intensity at every point.

There have been multiple studies that have attempted to calculate melt pool width in AM processes, but all of them have used emissivity based values to correlate the melt pool edge with a pixel value [10, 23-25, 27-31, 33, 34, 39, 40, 43-47, 67]. Other papers have claimed that using emissivity values in this way to correlate a pixel intensity value with temperature is not reliable $[39,40,47]$. This research has expanded on these claims to provide clear evidence. This research has provided fundamental thermographic knowledge in AM processes and has produced a new solution that calculates melt pool width based on the newly discovered edge features.

Whilst this research has uncovered some fundamental floors in emissivity based edge detection techniques, this should not cloud the substantial amount of advancements that the these technique have been used for. Conventional edge detection techniques have been used on multiple occasions to develop advanced AM control systems [23-25, 27, 28, 34, 45], as well as develop a further understanding of parameter interaction in AM builds $[23-25,27,28,34,45,47]$. The newly developed directional emittance algorithm could be used to further understand process parameters, develop enhanced control systems and improve clad quality in the same manner.

Research within this chapter highlighted some complex relationships in the AM process that haven't been documented. The rate at which DED clad tracks shrink during solidification could not be found in literature, but shrinkage rates in PBF processes are said to be influenced by both layer thickness and powder packing density [89]. Performing solidified track width measurements and comparing them to melt pool width measurements highlighted that the solidified track width is influenced by the laser power setting. Although both studies concern different processes, both this research and the referenced literature complement each other by suggesting that shrinkage rates can be influenced by process parameters.

Whilst results show multiple positive outcomes, there are limitations regarding the research conducted in this chapter. The major limitation when determining the accuracy of the melt pool di- 
mensioning system is that there is no way of performing a physical measurement of the melt pool itself. This research was able to determine that the directional emittance algorithm was more accurate than the conventional Canny edge technique, but there was no determination of how accurate the systems were compared to the true melt pool width value. Hand calculations are good indication of system accuracy, but further work should explore physical melt pool width measurements to improve confidence.

Studies have used melt pool monitoring systems to better understand the effects of parameter changes within AM processes [17, 23-25, 27, 28, 30, 33, 43, 45-49, 54]. Future work should utilise the new directional emittance algorithm, with improved accuracy, to provide a more detailed understanding of process parameter interactions in DED processes. Further work should study the effects that changing both PMFR and path velocity has on melt pool dimensions, as improved process understanding often leads to improved deposition quality [17, 23, 25, 43, 48, 49, 54].

Future work should also explore the use of the directional emittance algorithm on multiple materials. The newly developed technique is not reliant on material dependant emissivity values, meaning that the new technique should be able to perform melt pool calculations on new materials with little calibration. Subsequent research should use the directional emittance algorithm to directly compare two similar materials.

Conventional emissivity-based edge detection techniques do not accurately measure melt pool width. The newly developed directional emittance algorithm outperformed the Canny edge detection technique and was considered more accurate. This research has produced a melt pool dimensioning technique, with improved accuracy, that does not require material dependant emissivity values to perform calculations.

\subsection{Chapter Conclusion}

This chapter introduced a novel image processing technique that can more accurately measure the width of a melt pool when compared to the conventional Canny edge technique. The accuracy of the new directional emittance algorithm was tested by comparing measurements to hand calculations and performing single frame analysis. Measurements of solidified tracks were performed using an optical 
microscope, but it was discovered that the correlations between these and the melt pool could not be made due the complex changes in width that occur during the solidification stage.

The results highlighted fundamental issues with the Canny edge detection algorithm and showed that part of the melt pool edges often occur at different thresholds. The new directional emittance algorithm detects the edge of the melt pool using changes in intensity and is not subject to the same issues.

The new directional emittance algorithm combined advancements made in Chapter 3 to provide a robust system capable of calculating melt pool widths over a range of different laser power settings. The new algorithm has also eradicated the need for emissivity values, which are known to be of low accuracy.

The success of this newly developed system has allowed for a greater understanding of the DED process by highlighting the complex interaction between melt pool width and laser power setting. This information can be used by both academic and industrial staff to develop control systems and improve AM components. Melt pool monitoring systems have been used on multiple occasions to provide a greater understanding of the complex AM process and to optimise AM parameters for higher quality manufacturing.

Due to the system's success and initial prospects delivered in the form of greater process understanding, this system will be developed to allow for further use by academic and industrial members. 


\section{A Further Understanding of Laser Metal Deposition Sys- tems}

Chapter 4 detailed the design of a new melt pool dimensioning algorithm that can reliably measure melt pool width without the need for emissivity values. Though live melt pool measurements are useful for subjective defect detection by the machine operator, detailed melt pool analysis can be used for academic purposes to further understand the complex interactions that occur within the DED process. Previous literature has highlighted that the melt pool is one of the key parameters within the DED process and understanding how different parameters change this geometry can aid in the subsequent development of control systems. This knowledge can also be used to help operators more efficiently select optimum system parameters.

This work has shown that the new directional emittance algorithm can more accurately measure melt pool geometry and allowed for the construction of Figure 49, which showed how the melt pool width changes in relation to different laser power settings. This analysis extended the already established understanding that "increasing laser power increases melt pool size" to closely fit this understanding to a second order polynomial.

This chapter utilises the new directional emittance algorithm to provide more DED parameter studies. Literature has highlighted three parameters that majorly influence the behaviour of either the DED process, or the melt pool directly. These three parameters are laser power, path velocity and PMFR. The influence that laser power has on melt pool width has already been encapsulated in Chapter 4. This chapter further studies the complex melt pool interactions by using the same technique to establish how both path velocity and PMFR influence the width of the melt pool.

\subsection{The Influence of Powder Mass Flow Rate on Melt Pool Width}

PMFR is a parameter that is reported to majorly influence the melt pool in DED processes. Multiple papers have reported this $[13,14,23,25,27]$. This study aims to provide a more detailed understanding of this situation by using the new and improved melt pool dimensioning algorithm to distinguish melt 
pool width.

Achieving the correct PMFR in the DED process is key in producing a melt pool cladding track with the desired width. Having a low PMFR can lead to several issues when depositing using the DED method. Low PMFRs result in cladding tracks that have reduced solidified clad widths, which can result in incorrect deposited geometries. Low PMFRs can also result in reduced solidified clad heights. This could again result in manufactured components not meeting the geometric requirements (incorrect cladding thickness's). Reducing the PMFR also influences the amount of excess energy in the DED process. It is known that increasing the laser power will increase the amount of excess energy in the DED process and can lead to unwanted scenarios such as excessive dilution and keyhole formation. Decreasing the PMFR can also have this effect. If less powder is introduced to the melt pool, less laser power energy is used in melting the injected solid powder particles. This can result in excess energy melting more of the underlying substrate.

PMFR settings that are too high can also negatively influence the geometry of the deposited cladding tracks. If too much power is injected into the melt pool, larger melt pools can form resulting in components that do not meet the geometric requirements. Increasing the PMFR can also result in excess waste powder. For a given laser power setting, only a set amount of powder can be melted. The operator cannot expect to constantly increase the PMFR infinitely and expect all powder to be melted and absorbed into the melt pool. Increasing PMFR beyond a specific point will result in expensive excess powder being wasted, increasing the cost of components unnecessarily.

These issues are known to industrial staff but have not been studied in detail due to the lack of high quality melt pool analysis tools. With the introduction of the directional emittance technique, the study of how PMFR directly influences the geometry of the melt pool is carried out. This experiment aims to provide a detailed analysis of how PMFR influences melt pool width, to help industrial and academic staff optimise parameters. This work will aim to provide a better understanding of the DED process so that issues surrounding incorrect cladding geometry, excessive dilution and wasted powder can be reduced/eradicated. 


\subsubsection{Experimental Set-up}

This experiment was carried out using the set-up discussed in Section 3.4, with the machine depositing single line tracks with lengths of $150 \mathrm{~mm}$. The direction of travel was fixed, the carrier gas was set to $3.5 \mathrm{~L} / \mathrm{min}$, the shielding gas was set to $6 \mathrm{~L} / \mathrm{min}$, the path velocity was $11.25 \mathrm{~mm} / \mathrm{s}$, the laser spot size was $1.5 \mathrm{~mm}$, and three laser power settings of 750, 900 and $1050 \mathrm{~W}$ were used. All these settings remained consistent throughout the experiment. Using the IDS-imaging software, videos were recorded for multiple PMFR settings over three different laser power settings. The PMFR setting was iterated from $3.7-5.4 \mathrm{~g} / \mathrm{min}$ (9 to $12.5 \%$ on the machine) using increments of $0.25 \%$. The material used for deposition was EN25. Both the laser power and the PMFR were changed during this experiment to understand the influence of PMFR on melt pool width. The matrix of parameters used for the experiment are displayed in Table 5. Certain PMFR settings have multiple instances in the table due to some powder percentage values having the same $\mathrm{g} / \mathrm{min}$. The original powder percentage values used to perform this experiment can be found in section 3.4.5.

Table 5: The matrix of parameters used in the PMFR experiment.

\begin{tabular}{|c|c|c|c|c|c|}
\hline $\begin{array}{l}\text { Laser Power } \\
\text { (W) }\end{array}$ & $\begin{array}{l}\text { PMFR } \\
(\mathrm{g} / \mathrm{min})\end{array}$ & $\begin{array}{c}\text { Laser Power } \\
\text { (W) }\end{array}$ & $\begin{array}{l}\text { PMFR } \\
\text { (g/min) }\end{array}$ & $\begin{array}{l}\text { Laser Power } \\
\text { (W) }\end{array}$ & $\begin{array}{l}\text { PMFR } \\
(\mathrm{g} / \mathrm{min})\end{array}$ \\
\hline 750 & 3.7 & 900 & 3.7 & 1050 & 3.7 \\
\hline 750 & 3.9 & 900 & 3.9 & 1050 & 3.9 \\
\hline 750 & 4.0 & 900 & 4.0 & 1050 & 4.0 \\
\hline 750 & 4.2 & 900 & 4.2 & 1050 & 4.2 \\
\hline 750 & 4.3 & 900 & 4.3 & 1050 & 4.3 \\
\hline 750 & 4.3 & 900 & 4.3 & 1050 & 4.3 \\
\hline 750 & 4.5 & 900 & 4.5 & 1050 & 4.5 \\
\hline 750 & 4.7 & 900 & 4.7 & 1050 & 4.7 \\
\hline 750 & 4.8 & 900 & 4.8 & 1050 & 4.8 \\
\hline 750 & 4.8 & 900 & 4.8 & 1050 & 4.8 \\
\hline 750 & 4.9 & 900 & 4.9 & 1050 & 4.9 \\
\hline 750 & 5.0 & 900 & 5.0 & 1050 & 5.0 \\
\hline 750 & 5.2 & 900 & 5.2 & 1050 & 5.2 \\
\hline 750 & 5.2 & 900 & 5.2 & 1050 & 5.2 \\
\hline 750 & 5.4 & 900 & 5.4 & 1050 & 5.4 \\
\hline
\end{tabular}

Forty-five single line deposition tracks $150 \mathrm{~mm}$ in length were deposited using the parameters above 
and the optical system recorded all deposition activity. Forty-five video recordings were created for post process analysis and were subjected to the directional emittance image processing technique created in Chapter 4. The video recordings were then reprocessed and snippets were created using the same technique used in results Section 4.3.3. These snippets were used to achieve average melt pool width values for all parameter combinations displayed in Table 5.

\subsubsection{Results}

To easily compare the results of the above matrix, the average melt pool width for all forty-five recordings were plotted on a single graph, as depicted in Figure 58. The results from this plot provide information regarding the influence that PMFR has on melt pool width calculations for different laser power settings.

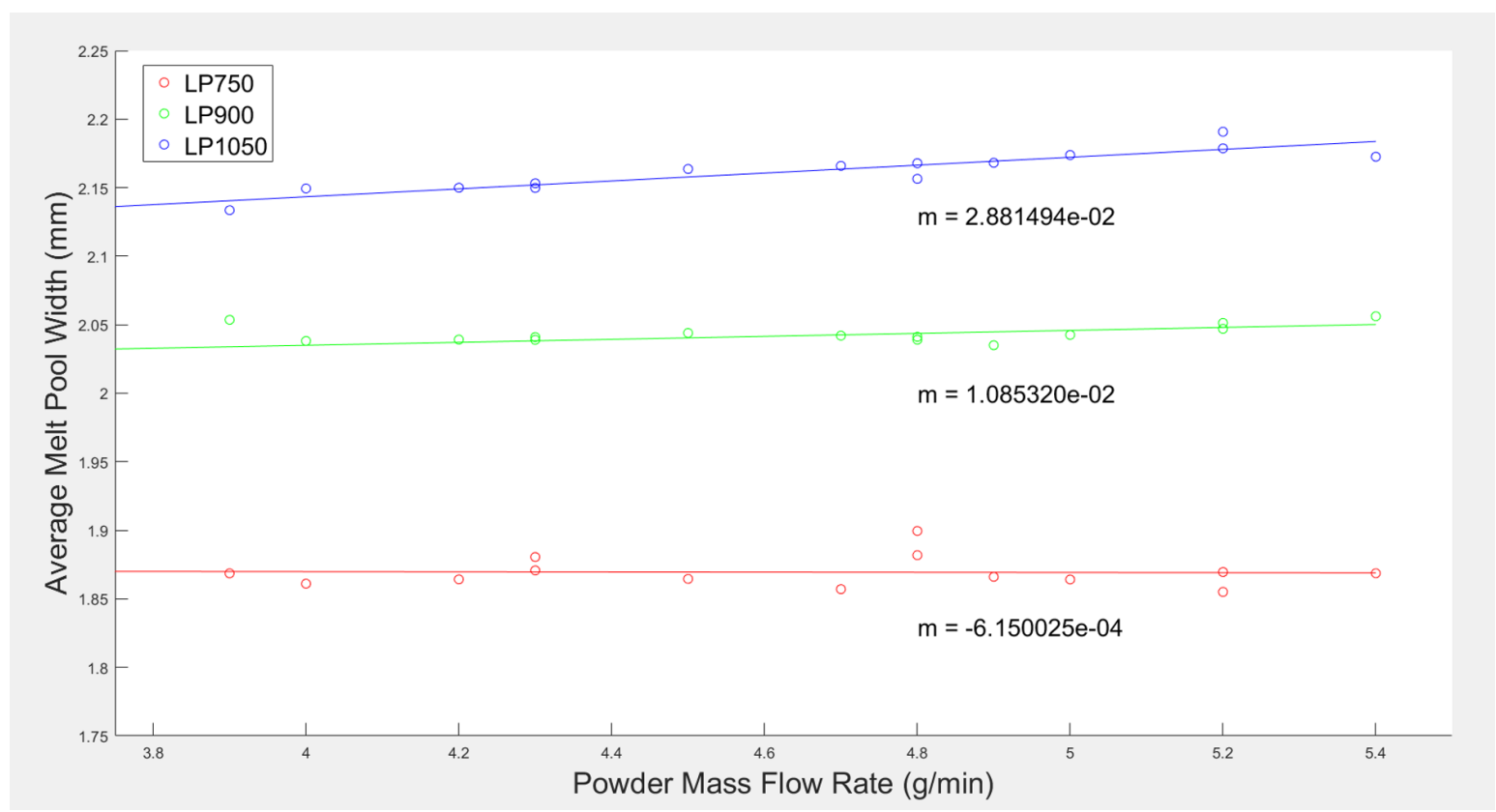

Figure 58: A graph representing all calculated average melt pool values for various parameter settings used in the PMFR experiment.

The effects that changing PMFR has on the melt pool width is dependent on the laser power, and it cannot simply be assumed that the melt pool width increases with increasing PMFR. For the 
highest laser power setting used in this experiment $(1050 \mathrm{~W})$ the melt pool width did increase when an increasing PMFR was used. Having a high laser power setting means that there is a more energy to melt powder and increasing the PMFR in this scenario resulted in more powder being melted and absorbed. This in turn led to an increase in melt pool width.

For the mid laser power setting this was again true. Having a relatively high laser power setting resulted in more powder being melted and accepted into the molten melt pool when the PMFR was increased. Both the mid and high laser power setting scenarios showed that there was excess energy to accept more powder to increase the width of the melt pool. The gradients of the lines for both the mid and high laser power settings differ. The tracks with a laser power setting of $900 \mathrm{~W}$ showed a much shallower increase in melt pool width for the same increase in PMFR. This is most likely due to there being insufficient energy to melt the extra powder as effectively.

The gradient of the line of best fit for the laser power setting of $750 \mathrm{~W}$ is very close to zero. The experimental results showed that increasing the PMFR for a lower laser power setting of $750 \mathrm{~W}$ did not increase the size of the melt pool. The average melt pool width stayed constant as the PMFR was increased. For tracks deposited using the lower laser power, there is larger deviation of calculated melt pool width values from the line of best fit. Using a laser power setting of $750 \mathrm{~W}$ showed more variance between melt pool width calculations which indicated melt pool instability.

The results from this graph clearly indicate the effects that changing PMFR has on the melt pool width cannot be generalised for all parameter settings. The effects that increasing/decreasing the PMFR has on the melt pool is dependent on other parameter settings used. On higher laser power settings there is more excess energy to melt additional powder allowing for the melt pool width to increase. For lower laser power settings, increasing the PMFR did not increase the width of the melt pool, and in-fact showed a decrease in melt pool width. For lower laser power settings there is not enough energy to melt excess powder, so adding more to the situation doesn't increase the size of the melt pool. The results from these graphs further highlight the complex influences that changing parameters have on melt pools in the DED process. The melt pool has shown to be a good indication of how certain parameters affect the DED process, and can be used for parameter optimisation. 


\subsubsection{Discussion}

This study aimed to provide a detailed understanding of how changes in PMFR can effect melt pool geometry. Studies have shown that PMFR is a parameter that is known to greatly effect DED processes $[13,14,23,25,27]$, but these studies have not explored the complex interaction between laser power, PMFR and melt pool width. This information can provide users with greater process understanding, which can subsequently lead to higher quality deposition. Understanding the intricacies of how different parameters interact with each other in the complex DED process is paramount when improving manufacturing capabilities.

This study used the new directional emittance algorithm to determine melt pool width at a range of different PMFR and laser power settings. Single line cladding tracks were deposited using the in house DED system and recorded for post process analysis. Average melt pool widths for each individual laser power and PMFR parameter combination were calculated and displayed in a single plot to allow for detailed analysis. The key finding from this study was that the relationship between laser power, PMFR and melt pool width is complex. The effects that changing PMFR has on melt pool width is dependent on the laser power setting used during deposition.

Previous literature sites the changes that PMFR has on melt pool temperature $[14,23]$, but the exploration of the effects of PMFR on melt pool dimensions has yet to be explored. Authors from both these references $[14,23]$ state that increasing the PMFR results in a decrease in overall temperature, but no further detail on the subject is given. Sun et al. and Meriaudeu et al. both explored the effects of PMFR on solidified track dimensions and stated that PMFR is one of the dominant features effecting these $[24,90]$. Their research shows that increasing the PMFR simply increases the size of the track width, but they do not detail the size of the melt pool throughout the process. Section 4.3.3.3 however details that there are variations between solidified and molten melt pool dimensions, resulting in no direct comparison to literature being obtainable. Results from this research have further explored the effects that changing PMFR has on melt pool behaviour and has revealed new detail on the complex relationship.

The major limitation of this study is the measurement of PMFR. PMFR was set by selecting a 
percentage value on the DED system, which determined how much of the exit hole on the hopper was open. Previous work (Section 3.4.5) has detailed the correlation between the exit hole opening percentage and $\mathrm{g} / \mathrm{min}$ value for the powder delivery, but this value will most likely differ when compared to the actually $\mathrm{g} / \mathrm{min}$ delivered to the melt pool in an instance. Nozzle blockages, variations in powder delivery gas flow and random powder particle distributions will likely cause changes to the PMFR that currently go unnoticed. Measuring the PMFR after it has exited the nozzle will allow for a deeper understanding of the complex parameter interaction and will likely increase confidence in experimental results.

Future work should implement a more detailed PMFR measurement tool to further validate these discoveries. In conjunction with this, a larger range of laser power parameters should be used to further understand the complex interaction between laser powder, PMFR and melt pool width. As more detailed parametric studies are conducted and more data is collected, a new way of analysing data should be explored. Ultimately this data should be linked to track quality and even the resulting microstructure. Using this data with advanced control systems could then allow users to control quality, microstructure and even mechanical properties throughout DED processes.

This research has proved that the new directional emittance algorithm can be used to grant a detailed understanding of the relationship between laser power, PMFR and melt pool width in DED processes. It was discovered that the relationship between these three parameters is complex, and the effects that changing PMFR has on melt pool width is dependant on the laser power setting used during deposition.

\subsubsection{Conclusion}

The newly developed image processing technique was successfully able to measure the melt pool width for various PMFRs and was able to distinguish trends with changing parameters. Increasing the PMFR increased the melt pool width for the higher laser power settings (1050W and 900W) but did not increase the melt pool width for the lower laser power setting $(750 \mathrm{~W})$. The results suggest that a complex relationship between the laser power, PMFR and melt pool width exists. Future work should study this relationship in more detail. 


\subsection{The Influence of Path Velocity on Melt Pool Width}

Path velocity is an AM parameter that has been documented multiple times to directly influence melt pool geometry and the deposited material $[13,15,17,47,86,91]$. Path velocity is an important parameter in the DED process, and increasing/decreasing this value can have both benefits and drawbacks. Finding a balanced path velocity can lead to optimum part quality and reduced deposition times.

Increasing the path velocity in the AM process has the obvious benefit of reducing the amount of time it takes for manufacturing. Increasing the path velocity can allow for an increase in both laser power and PMFR to subsequently increase the amount of material deposited over a period of time. Though benefits can come from increasing the path velocity, increasing the path velocity can also result in the destabilisation of the melt pool. Increasing path velocity can cause melt pool formation to be poor and result in a lack of fusion between the deposited material and the underlying substrate. This study aims to establish how melt pool width is affected by changes in path velocity.

\subsubsection{Experimental Set-up}

This experiment was carried out using the set-up discussed previously in Section 3.4, with the machine depositing single line tracks with lengths of $150 \mathrm{~mm}$. The direction of travel was fixed, the carrier gas was set to $3.5 \mathrm{~L} / \mathrm{min}$, the shielding gas was set to $6 \mathrm{~L} / \mathrm{min}$, the laser spot size was $1.5 \mathrm{~mm}$, and three laser power settings of 750, 900 and $1050 \mathrm{~W}$ were used. The PMFR setting was set to $4.8 \mathrm{~g} / \mathrm{min}$. The material used for deposition was EN25. All these settings remained consistent throughout the experiment. The settings were selected due to them producing stable melt pools. Using the IDS-imaging software, videos were recorded for multiple path velocity settings over three different laser power settings. The path velocity setting was iterated from 9.5 to $13 \mathrm{~mm} / \mathrm{s}$ with a total of fifteen increments $(0.25 \mathrm{~mm} / \mathrm{s})$. Both the laser power and the path velocity were changed during this experiment to understand the influence of path velocity on melt pool width. The matrix of parameters used for the experiment are displayed in Table 6 . 
Table 6: The matrix of parameters used in the path velocity experiment.

\begin{tabular}{|c|c|c|c|c|c|}
\hline $\begin{array}{l}\text { Laser Power } \\
\text { (W) }\end{array}$ & $\begin{array}{l}\text { Path Vel. } \\
(\mathrm{mm} / \mathrm{s})\end{array}$ & $\begin{array}{l}\text { Laser Power } \\
\text { (W) }\end{array}$ & $\begin{array}{l}\text { Path Vel. } \\
(\mathrm{mm} / \mathrm{s})\end{array}$ & $\begin{array}{l}\text { Laser Power } \\
\text { (W) }\end{array}$ & $\begin{array}{c}\text { Path Vel. } \\
(\mathrm{mm} / \mathrm{s})\end{array}$ \\
\hline 750 & 9.5 & 900 & 9.5 & 1050 & 9.5 \\
\hline 750 & 9.75 & 900 & 9.75 & 1050 & 9.75 \\
\hline 750 & 10 & 900 & 10 & 1050 & 10 \\
\hline 750 & 10.25 & 900 & 10.25 & 1050 & 10.25 \\
\hline 750 & 10.5 & 900 & 10.5 & 1050 & 10.5 \\
\hline 750 & 10.75 & 900 & 10.75 & 1050 & 10.75 \\
\hline 750 & 11 & 900 & 11 & 1050 & 11 \\
\hline 750 & 11.25 & 900 & 11.25 & 1050 & 11.25 \\
\hline 750 & 11.5 & 900 & 11.5 & 1050 & 11.5 \\
\hline 750 & 11.75 & 900 & 11.75 & 1050 & 11.75 \\
\hline 750 & 12 & 900 & 12 & 1050 & 12 \\
\hline 750 & 12.25 & 900 & 12.25 & 1050 & 12.25 \\
\hline 750 & 12.5 & 900 & 12.5 & 1050 & 12.5 \\
\hline 750 & 12.75 & 900 & 12.75 & 1050 & 12.75 \\
\hline 750 & 13 & 900 & 13 & 1050 & 13 \\
\hline
\end{tabular}

Forty-five single line deposition tracks of length $150 \mathrm{~mm}$ were deposited using the parameters above and the optical system recorded all deposition activity. Forty-five video recordings were created for post process analysis and were subjected to the new image processing technique created in Chapter 4 . The video recordings were then reprocessed and snippets were created using the same technique used in Section 4.3.3. These snippets were used to achieve average melt pool width values for all parameter combinations displayed in Table 6.

\subsubsection{Results}

To easily compare the results of the above matrix, the average melt pool width for all forty-five recordings were plotted on a single graph. The graph displaying the results is depicted in Figure 59 . The results from this plot provide information regarding the influence that the path velocity setting has on calculated melt pool widths.

For all laser power settings, the average melt pool width decreased when the path velocity was increased. The melt pool width increases when the laser power setting is increased due to higher thermal gradients and larger physical forces exerting themselves along the length of the melt pool. 


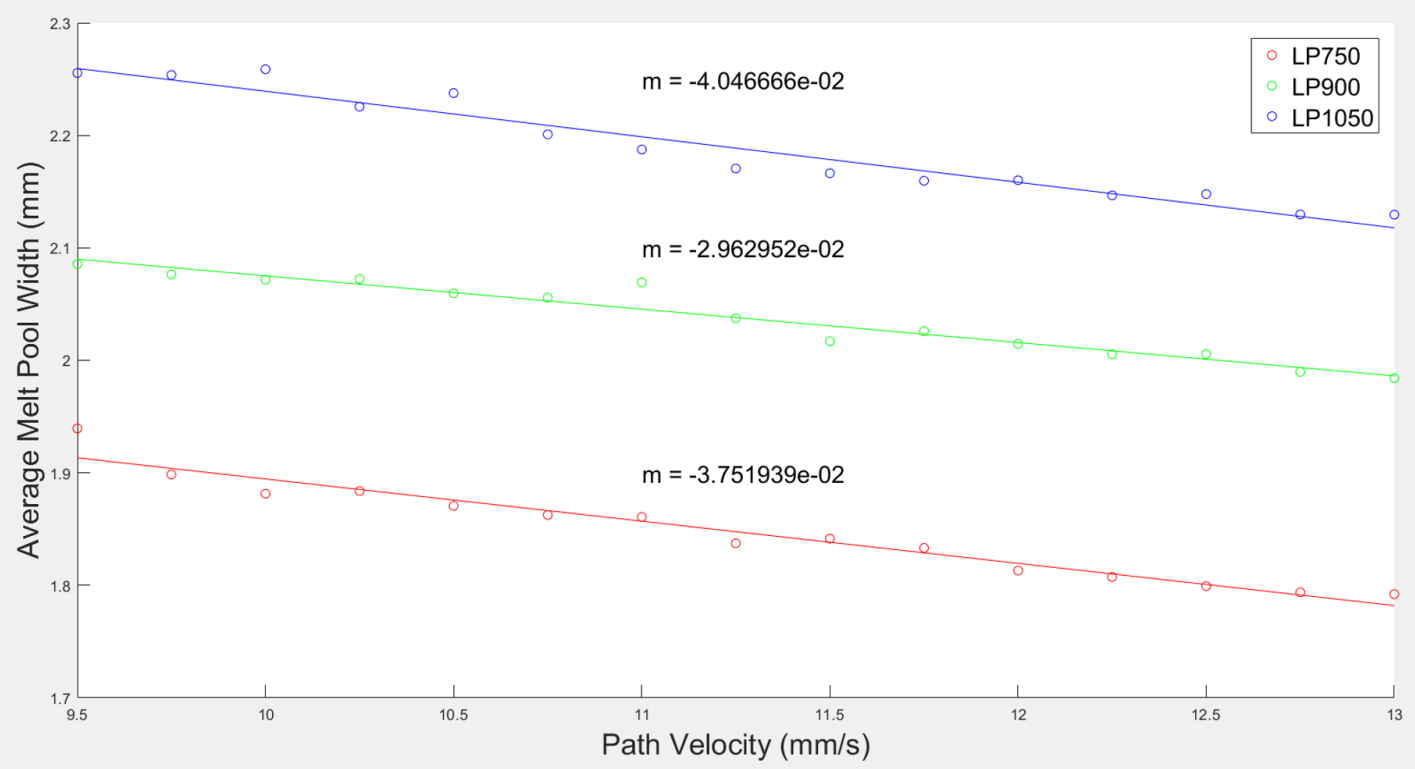

Figure 59: A graph representing all calculated average melt pool values for various parameter settings used in the path velocity experiment.

Increasing the path velocity also results in a decrease in energy density, which in turn results in less power being able to melt the molten powder and substrate to form a wider melt pool.

The gradients of the three lines of best fit have also been displayed on the graph. The lines of best fit all show a negative gradient of similar values. This said, performing the cladding tracks at a laser power of $900 \mathrm{~W}$ showed the shallowest gradient. The difference between this gradient and the other two could be a result of the complex interactions that occur between different parameters. Increasing the path velocity could have different results on the melt pool geometry depending on other parameters that are used in the process. The difference in gradients between these lines are not prominent enough to be able to draw conclusions and more research should be performed to further increase understanding. This can be done by performing the same experiment but increasing the number of iterations of recorded videos over different laser power settings. 


\subsubsection{Discussion}

This experiment aimed to determine how melt pool width changes with different path velocity and laser power settings. Previous literature has used melt pool dimensioning algorithms, with constant pixel intensity values, to determine the edge of the melt pool $[10,23-25,27-31,33,34,39,40,43-$ 47, 67], but these techniques were proved to be inaccurate in previous research (Chapter 4). This experiment was designed to establish whether the new directional emittance algorithm could validate documented results and potentially uncover more detail about how the melt pool changes with varying path velocities. Developing an enhanced understanding of the interaction between laser power, path velocity and melt pool geometry could subsequently lead to improved control systems, higher quality deposition and optimised build times.

This study used the new directional emittance algorithm to determine melt pool width at a range of different path velocity and laser power settings. Single line cladding tracks were deposited using the in house DED system and recorded for post process analysis. Average melt pool widths for each individual laser power and path velocity parameter combination were calculated and displayed in a single plot to allow for detailed analysis. This study was unable to discover any new relationships between laser power, path velocity and melt pool width that have yet to be documented. For all laser power settings, the average melt pool width reduced in size when the path velocity was increased.

Results from this experiment compliment those that have been previously documented. Meriaudeu et al. details the effects that path velocity has on solidified track width and reports that increasing path velocity results in a decrease in track width [24]. Hua et al. details the effects that path velocity has on melt pool temperature. Their results show that increasing the path velocity reduces the temperature of the melt pool during manufacturing [14]. Cheng et al. details the effects of path velocity on melt pool width, and indicates that increasing path velocity results in a decrease in melt pool width [47]. Although all three of these studies measure the effects of path velocity on different parameters, the melt pool width, melt pool temperature and solidified track width are undoubtedly linked. This indicates that scan speed effects melt pool width, melt pool temperature and solidified track width in a similar manner. The experimental results complement what has been published previously in 
literature, demonstrating that the directional emittance algorithm can be used to study parameter interaction.

This study showed that there is a strong relationship between increasing path velocity and decreasing melt pool width. This study also showed that for all the laser power use cases, this statement was true. The limitations of this however are that there are only three laser power settings used in the experiment. The relationship between laser power, path velocity and melt pool width seems apparent in this research, but performing the experiment with more laser power settings could uncover a more detailed relationship.

Future work should look to expand the amount of parameter use cases and increase the total amount of data. Increasing the amount of data collected will allow for the academic solidification of the relationship between path velocity and melt pool width. Subsequent studies should look to then determine whether this relationship is true when changing other parameters such as PMFR, path geometry and even material. This large collection of data should be used to develop advanced control systems that can control multiple parameters throughout builds to optimise deposition quality.

This research has verified, using the new directional emittance technique, that increasing path velocity results in a decrease in melt pool width. This was shown to be the case for three different laser power settings when depositing EN25. This study validates the previously documented understanding of the interaction between melt pool width and path velocity by using a more accurate melt pool dimensioning algorithm and multiple laser power settings.

\subsubsection{Conclusion}

The new directional emittance technique was used in a parametric study to understand the influence that path velocity has on melt pool width. Increasing path velocity resulted in a decrease in melt pool width for all laser power settings. Variations between gradients were evident in the experiment, but no conclusion could be drawn from these differences due to the lack of laser power cases. Further investigations should look to compare more iterations over different laser power settings, to further understand the complex interaction of parameters in the DED process. 


\subsection{Chapter Conclusion}

This chapter implemented the new directional emittance image processing technique (developed in Chapter 4) to further understand process parameter interactions in the DED process. Two separate parameter studies were conducted using the developed technique to determine how these parameters affect the width of the melt pool in the DED process. The directional emittance algorithm was able to more accurately measure the melt pool and produce a more detailed analysis of how both the PMFR and path velocity influence the melt pool's width.

Increasing the PMFR when there is excess energy available (using higher laser power settings) results in the melt pool width increasing as more powder is melted by the excess energy. In this case a larger body of molten material was formed. This was evident on the mid-level laser power settings (900W), but the influence that increasing PMFR had on the melt pool width was reduced when compared with the high laser power setting (1050W). Performing this experiment on the lowest level laser power setting $(750 \mathrm{~W})$ showed results that have not been documented previously and increasing the PMFR at this laser power setting resulted in no increase in melt pool width. Low energy levels were not able to melt the extra powder that was introduced, and resulted in the molten material not growing in size.

Increasing the path velocity showed a decrease in melt pool width for all laser power settings. Differences between the gradients of lines of best fit indicated that the effects of decreasing path velocity has on the melt pool width vary for different parameter settings, but the differences were not as prominent as those displayed in the previous PMFR study. No conclusion could be drawn from this data due to the lack of cases (three laser power settings) and the subtle differences between them. The conclusion drawn from this experiment is that increasing path velocity results in the melt pool width decreasing, but subsequent work should investigate this interaction further.

The directional emittance algorithm has been successfully used to better understand the complex DED process, and has uncovered how certain parameters influence the melt pool in more detail. Research from this chapter, combined with the preliminary laser power study in Chapter 4 has outlined how the laser power, path velocity and PMFR settings effect the melt pool width in DED processes. 
The conclusion drawn from these results is that these three main parameters have a complex interaction with one another and changing one can directly influence how the melt pool reacts to further parameter changes. The new directional emittance algorithm has shown its use in the field of DED as it can provide a more detailed melt pool analysis than conventional emissivity based edge detection techniques. The new algorithm should be used to further study these complex parameter interactions and be used to build complex control systems that can tightly manipulate multiple parameters throughout DED build cycles simultaneously. 


\section{Melt Pool Monitoring of Different Materials}

The new melt pool monitoring system has seen the development of new hardware concepts and algorithms. The new system has been used for academic purposes to better understand EN25 melt pools by conducting a series of parametric experiments. The three main parameters that were chosen to be manipulated in the DED process were the laser power, PMFR and path velocity. The new studies have shown that the interaction between these three parameters, independent of the vast amount of other parameter interactions, is incredibly complex.

The new directional emittance algorithm has allowed for more accurate measurements of the melt pool without the need for emissivity values. Calculating emissivity values for molten metals is incredibly difficult, and it is documented that values are unreliable and inaccurate $[39,40,47]$. The eradication of emissivity values and the introduction of image processing methods that are based on physical features has not only increased the reliability of measurements, but has also opened opportunities to use the new image processing technique to measure different materials. This chapter will develop a calibration procedure that can be used to set up the melt pool monitoring system to measure different materials. This chapter will test the reliability of the melt pool calculations by directly comparing them to images taken from video recordings.

Being able to perform accurate melt pool width measurements on multiple materials without the need for lengthy prior experimentation will allow this system to be used to compare different materials. In a production environment, new materials are often introduced to the DED machine without parameter guidance. The understanding of a material is usually conducted via a trial and error process, using excessive powder and wasting time. A melt pool monitoring system that can accurately measure new powders with little calibration can aid industrial staff in quickly understanding what parameters are optimum for deposition.

To test the feasibility of these statements, a new material was chosen for deposition in this chapter. To limit the number of variables changed in the study, it was decided that deposition should be performed on the same substrate used for all previous experiments. The powder to be introduced in this chapter therefore needed to be compatible with the substrate used in previous ones. A commonly 
used powder in the DED process is Stainless Steel 316L and is the material selected to test the new system. The composition of this material is displayed in Table 7 .

Table 7: Chemical composition of SS 316L powder used in Chapter 6 deposition

\begin{tabular}{lc}
\multicolumn{1}{c}{ Element } & wt\% \\
\hline Carbon & 0.012 \\
Molybdenum & 2.5 \\
Nickel & 12.4 \\
Iron & Balance \\
Manganese & 1.5 \\
Chromium & 16.5 \\
Silicon & 0.6
\end{tabular}

Minor calibrations are made to the monitoring system to allow for melt pool width calculations of multiple materials. This chapter uses the newly developed algorithm to directly compare two different materials together.

This chapter will encompass the methodology and procedures used to calibrate the current melt pool monitoring system to be used on the different materials.

\subsection{Calibration For New Materials}

The new material was introduced into the hopper for experimental deposition. The same camera set-up that has been used for all experiments (explained in Section 3.4) was used to view the melt pool upon deposition. The same exposure times for the given laser power settings were used when depositing with the new 316L powder.

Upon deposition and initial experimental recordings it was found that melt pool images were over saturated with the given exposure time settings. Using the same exposure times that were chosen by the operator in Chapter 3 for EN25 led to all images at all laser power settings being over saturated. Figure 60 compares individual melt pool frames when depositing with EN25 and 316L for multiple laser power settings. 

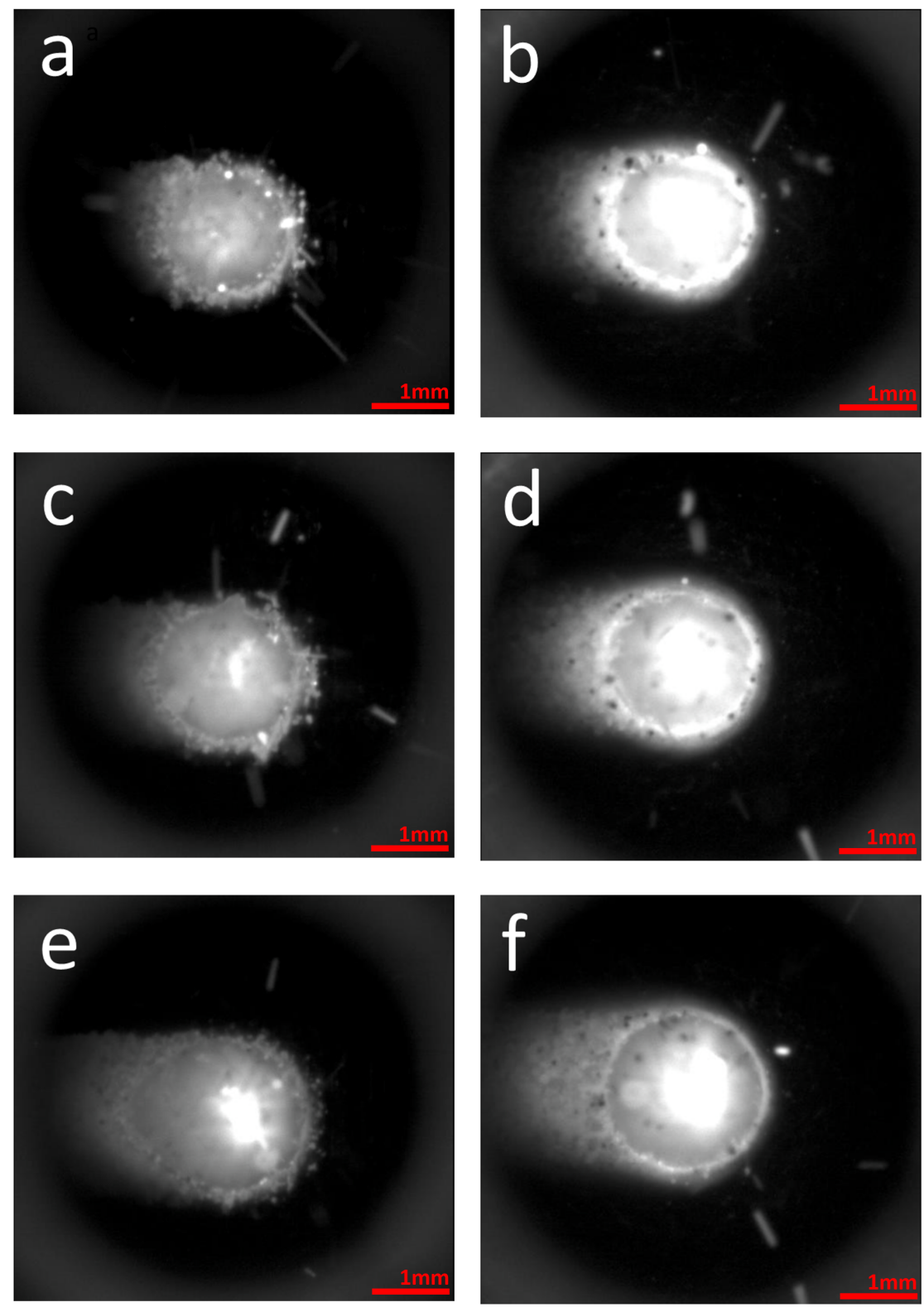

Figure 60: A series of images comparing melt pool saturation levels for a range of different laser power settings. $\mathrm{a} \& \mathrm{~b}=600 \mathrm{~W}, \mathrm{c} \& \mathrm{~d}=750 \mathrm{~W}$, e\&f $=900 \mathrm{~W}$. All melt pools on the left are EN25 melt pools and all melt pools on the right are $316 \mathrm{~L}$ melt pools. 
The saturation of images occurs due to the difference in powder, which can be a result of multiple factors. The first factor is that 316L has a different melting temperature to EN25. Different melting temperatures result in different radiation levels at the same laser power setting. Different radiation levels subsequently cause different pixel intensity readings and could be an explanation for the high saturation levels in the $316 \mathrm{~L}$ melt pool images.

The size of the melt pool also changes between the two materials. Larger melt pools cause larger portions of saturated pixels for fixed exposures times due to a larger surface area contributing radiation at maxima (the directional emittance phenomena). This said, the larger of the two melt pools for a given laser power settings was EN25, suggesting that 316L deposition recordings should have less saturation. Whilst this is clearly not the case, the effect of surface area should still be considered even if it is overcompensated by other contributing factors.

Another factor that can influence the amount of radiation received by the CMOS sensor is the powder particle size. Smaller powder particles tend to melt quicker in the melt pool as they have a larger surface area to volume ratio. Powder with courser powder particles will likely remove more energy from the melt pool area to fully melt individual particles, causing less excess radiation emitting in the direction of the CMOS sensor.

The high levels of image saturation will likely cause errors when the image processing algorithm developed in Chapter 4 is implemented on the video recordings. For this reason, the exposure time settings on the camera was changed and a new method was developed to allow for better quality imagery of melt pools made from different materials.

\subsubsection{Methodology for Calibrating Different Materials}

The new methodology uses the simple straight line approximation similar to the one developed in Chapter 3. The user selects the range of laser powers that they desire to operate in and then optimises the exposure time based on the original observational criteria highlighted in Section 3.7.2. This optimisation process is performed on the lowest and highest laser power settings to be used. The user will then use basic interpolation between these two exposure times to calculate exposure times for all laser power settings. This methodology was used to calibrate the cameras exposure time setting for 
the experimental study of $316 \mathrm{~L}$.

\subsubsection{Experimental Methodology}

This experiment was carried out using the set-up discussed previously in Section 3.4, with the machine depositing single line tracks with lengths of $150 \mathrm{~mm}$. The direction of travel was fixed, the carrier gas was set to $3.5 \mathrm{~L} / \mathrm{min}$, the shielding gas was set to $6 \mathrm{~L} / \mathrm{min}$, the path velocity was $11.25 \mathrm{~mm} / \mathrm{s}$, the laser spot size was $1.5 \mathrm{~mm}$ and the PMFR was set to $4.8 \mathrm{~g} / \mathrm{min}$. All these settings remained consistent throughout the experiment. The material used for deposition was 316L. Using the IDSimaging software, videos were recorded for multiple laser power settings and exposure times. The laser power setting was iterated from $600 \mathrm{~W}$ to $1050 \mathrm{~W}$ with a total of sixteen increments (every $30 \mathrm{~W}$ ). The laser powers for this experiment were narrowed due to the smaller operating window of the material.

To select the correct exposure times, multiple cladding tracks were deposited using the laser power settings of $600 \mathrm{~W}$ and $1050 \mathrm{~W}$. Whilst these tracks were being deposited, suitable exposure time settings were selected using the criteria defined in Section 6.1.1. Using this method, exposure times were created for all laser power settings. These were the exposure times used to record melt pool videos throughout track deposition.

Once all videos had been recorded, the video data was analysed using MATLAB for ease of data representation. All videos were subjected to the directional emittance algorithm and responses for all frames were documented. Snippets were then taken from the melt pool width measurements so that accurate melt pool width averages could be calculated. Details of how snippets were created are explained in Section 4.3.3.

\subsubsection{Results}

\subsubsection{Original Algorithm Run}

Running the original algorithm on the new videos resulted in non-optimal melt pool measurements. The new directional emittance algorithm was implemented on all frames and the operator observed the videos with superimposed north and south locations on the original image feed. It was quickly observed that a section of the frames being processed had incorrect melt pool measurements. Conducting the 
post process analysis using the original algorithm showed that correct measurements could be achieved, but alterations to the code had to be made to make measurements more accurate for all frames. To provide a more detailed analysis of what was happening, diagrams representing certain stages of the algorithm were created. This section provides an analysis of the incorrect measurements received from the new melt pool dimensioning system, and the corrective actions taken.

Breaks were placed throughout the video analysis to stop the algorithm on frames that gave incorrect measurements. The frames that were producing incorrect measurements were correctly analysing the melt pool's north location but were producing south locations that were outside the melt pool. The video recordings that were being used were of good standard, the melt pool showed low levels of pixel saturation in the centre of the melt pool, and the directional emittance phenomena was present.

The cause of the algorithm failing was found to be the calculation of the upper and lower thresholds. The frames that had incorrect measurements overestimated the melt pool due to the directional emittance feature not being located between the upper and lower threshold windows. The equations to calculate the upper and lower threshold are 12 and 13 respectively. For ease of explanation, the smoothed signal used to calculate the north and south locations for an incorrect frame is displayed in Figure 61 . The figure displays a smoothed signal with the directional emittance phenomena outside the threshold windows. The frame that the signal was taken from is displayed in Figure 62 with the measured north and south locations indicated by the dotted red lines. Dotted lines are used in this diagram to allow for easy viewing of the underlying signal/melt pool image.

Figure 61 shows signal characteristics that were not present in previous EN25 images that can cause difficulties in melt pool width extractions. The signal in Figure 61 is far more erratic, which is thought to be caused by differences in material properties. The weldability of $316 \mathrm{~L}$ is considered to be less than that of EN25 meaning that there are likely to be more geometrical fluctuations when using the new material. Differences in chemical compositions are likely to cause changes in melt pool behaviour, and subsequently the extracted signals from melt pool images. The kin $\mathrm{k}$ in the signal that directly relates to the melt pool edge is clouded by noise in the new material.

When the directional emittance feature is outside the threshold window it will not be found in the melt pool north/south location calculation. This will lead to incorrect measurements. The threshold 

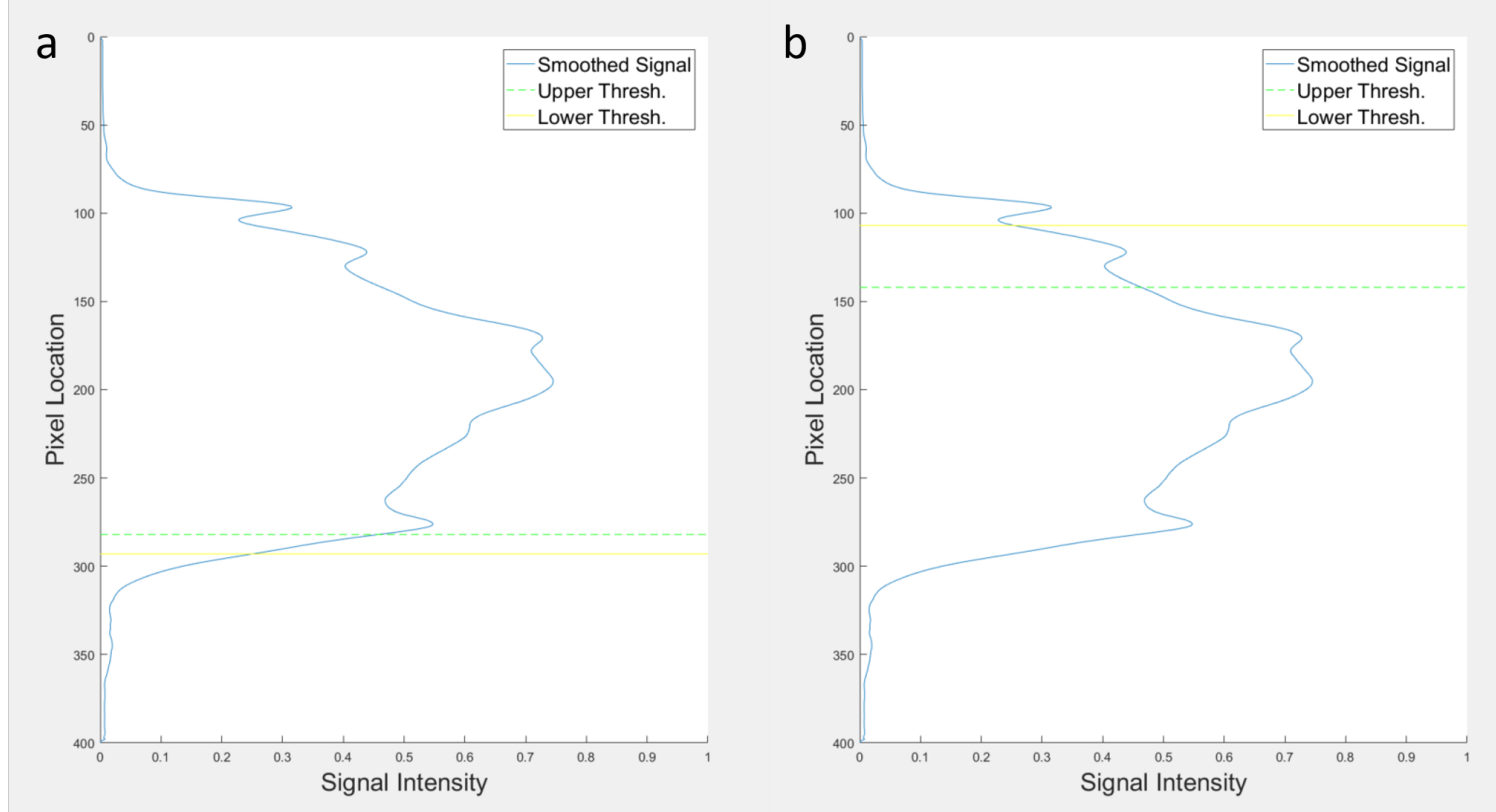

Figure 61: A graph representing part of the image processing algorithm. The smooth signal is extracted from the incorrectly measured frame, and the upper and lower thresholds used to extract the directional emittance signal are displayed. a) Thresholds attempting to measure the south location. b) Thresholds attempting to measure the north location.

window is calculated using Equations 12 and 13, which are dependent on a central intensity calculation. The central intensity value is calculated by taking a section of values of the smoothed signal and averaging them. It should be noted that the central intensity is directly influenced by the exposure time of the camera.

The location of the directional emittance phenomena does not change with different exposure time settings, but the locations of the upper and lower threshold values do. Selecting the correct exposure time is a difficult task to do quantitatively, and for the single material, the straight line approximation was adequate for selecting the correct exposure time. Using the operator's criteria defined in the original experiment for adaptive exposure times (3.7.2) has however led to incorrect measurements of the melt pool using the developed algorithm for the material 316L.

Though the algorithm failed to compute on certain frames in the video recordings for the new $316 \mathrm{~L}$ 


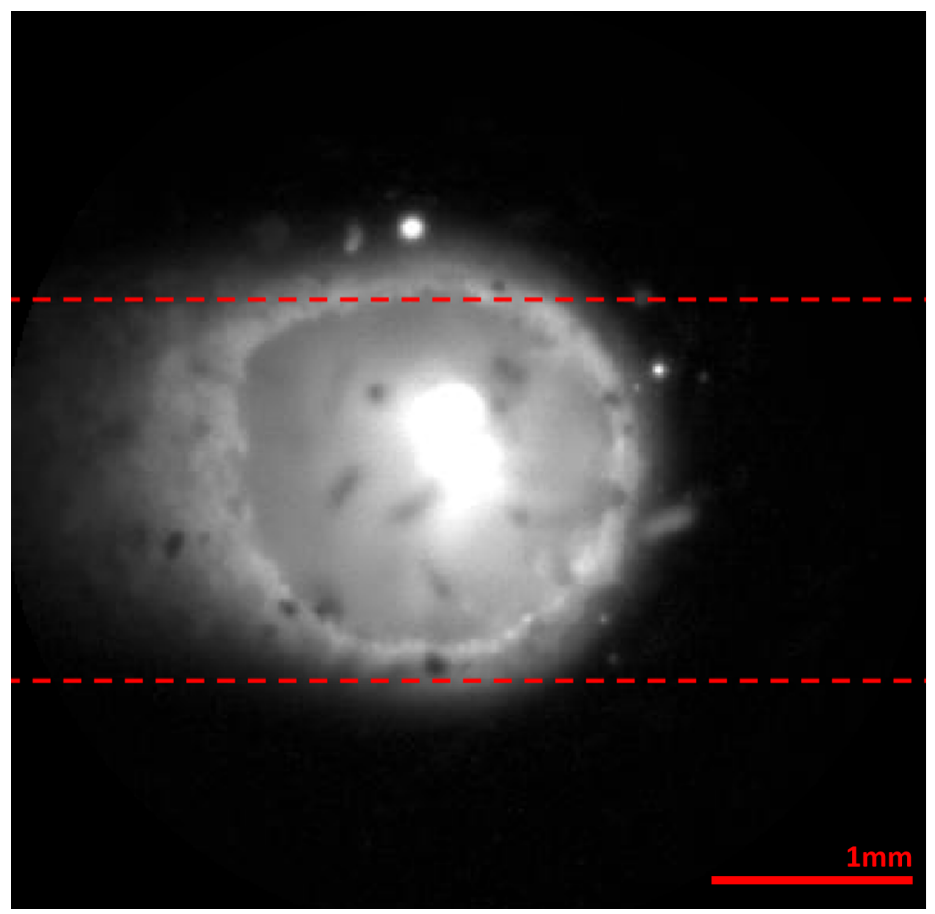

Figure 62: A melt pool image frame that gave an incorrect south location.

material, frames that are correctly measured show potential for the directional emittance algorithm. Frames that were correctly measured showed the directional emittance phenomena between the upper and lower threshold values when the smoothed signal was used for the calculation of the central intensity.

The locations of these windows can be changed by altering some of the fixed variables in Equations 12 and 13 . In these equations there are four fixed variables $\left(U_{\min }, U_{\max }, L_{\max }\right.$ and $\left.L_{\min }\right)$ that directly influence the values calculated for the upper and lower thresholds. The values used for EN25 experiments were adequate for all laser power settings, but these have shown to produce threshold values that do not reliably encapsulate the directional emittance phenomena for 316L. Changing these user defined values resulted in more reliable melt pool measurements and drastically increased the number of correctly analysed frames. The new values were then used to analyse the $316 \mathrm{~L}$ video recordings. These results are documented in Section 6.1.3.2. 
The user defined variables and methodology to select the correct exposure time for a given melt pool scenario have shown their limitation in this example. Whilst data from Section 6.1.3.2 will highlight how modifying the user defined variables can achieve reliable results, this workaround detracts some of the key benefits of using this newly developed system. The complexity of this problem, the limitations of the current algorithm and the potential use in further applications is analysed in more detail in the future work (Chapter 8).

\subsubsection{Optimised Algorithm Run}

The four user defined values $\left(U_{\min }, U_{\max }, L_{\max }\right.$ and $\left.L_{\min }\right)$ were changed, resulting in the upper and lower threshold moving to encompass the directional emittance phenomena. No other alterations were made on the algorithm. The same video recordings of the deposition defined in the methodology section (Section 6.1.2) were used to extract values for the melt pool width. The melt pool width average size was calculated using the techniques explained in Section 6.1.2.

Slightly altering the algorithm resulted in accurate melt pool width calculations. Observations of the algorithm's execution were carried out and it was found that very few (if any) incorrect measurements were apparent. Altering the position of the window using the user defined values resulted in the directional emittance phenomena being between the upper and lower threshold windows. Snippets were extracted from the videos and are displayed for range of laser power settings in Figure 63.

The snippets show stable measurements from the melt pool width. The graph displays slight variations in the melt pool width across the frames, which is expected due to fluctuations in the process. The signal from the highest laser power settings show some deviations from the stable melt pool response and was a result of the directional emittance phenomena being outside the threshold windows. It should be noted that this is a causation of a poorly selected exposure time from the operator causing a very dark melt pool image. Figure 64 compares two melt pool images, both with the same laser power setting. The image on the left is from a recording of EN25 deposition, the image on the right is from a recording of 316L deposition. Directly comparing these two images together further explains some of the difficulties that arose using operator's criteria to select exposure times.

Some frames for the higher laser power settings gave incorrect melt pool dimensioning readings, 


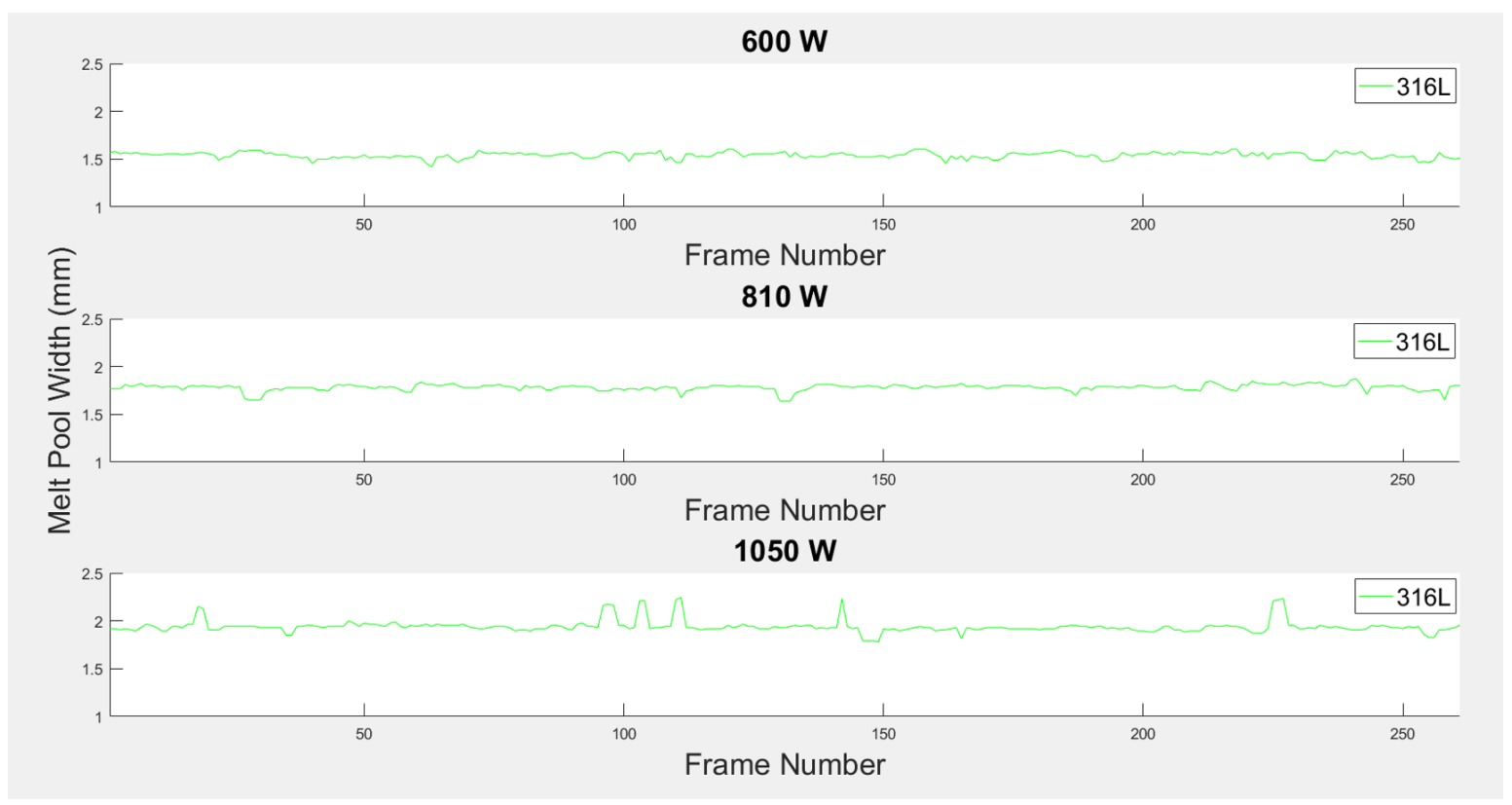

Figure 63: Snippets extracted from the melt pool width calculations for tracks depositing at various laser power settings.
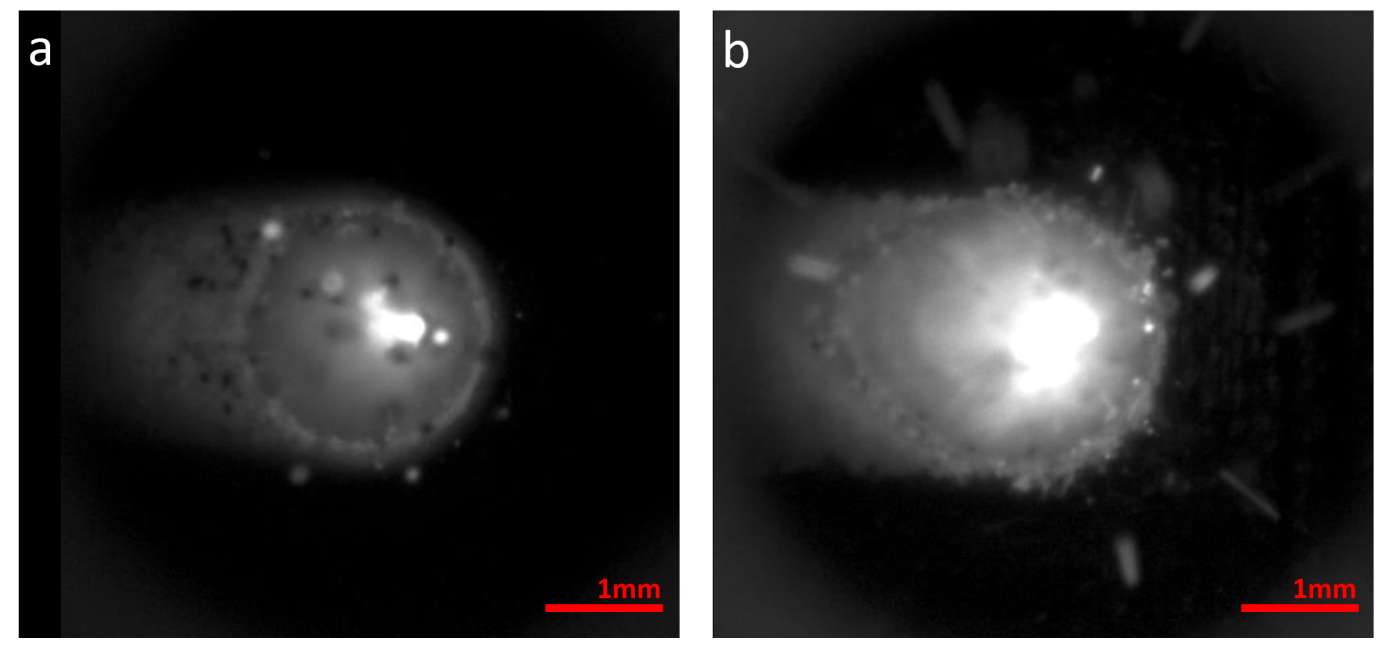

Figure 64: a: A still image taken using the exposure time selected by the operator for the material 316L. b: A still image taken using the exposure time selected by the operator for the material EN25. The laser power setting for both images is $900 \mathrm{~W}$. 
but due to the infrequency of these readings, the snippets were still used to calculate average melt pool widths for the laser power settings. The average melt pool widths were then plotted to observe how melt pool width changes for different laser power settings when depositing with 316L powder. The average melt pool width results are displayed in Figure 65.

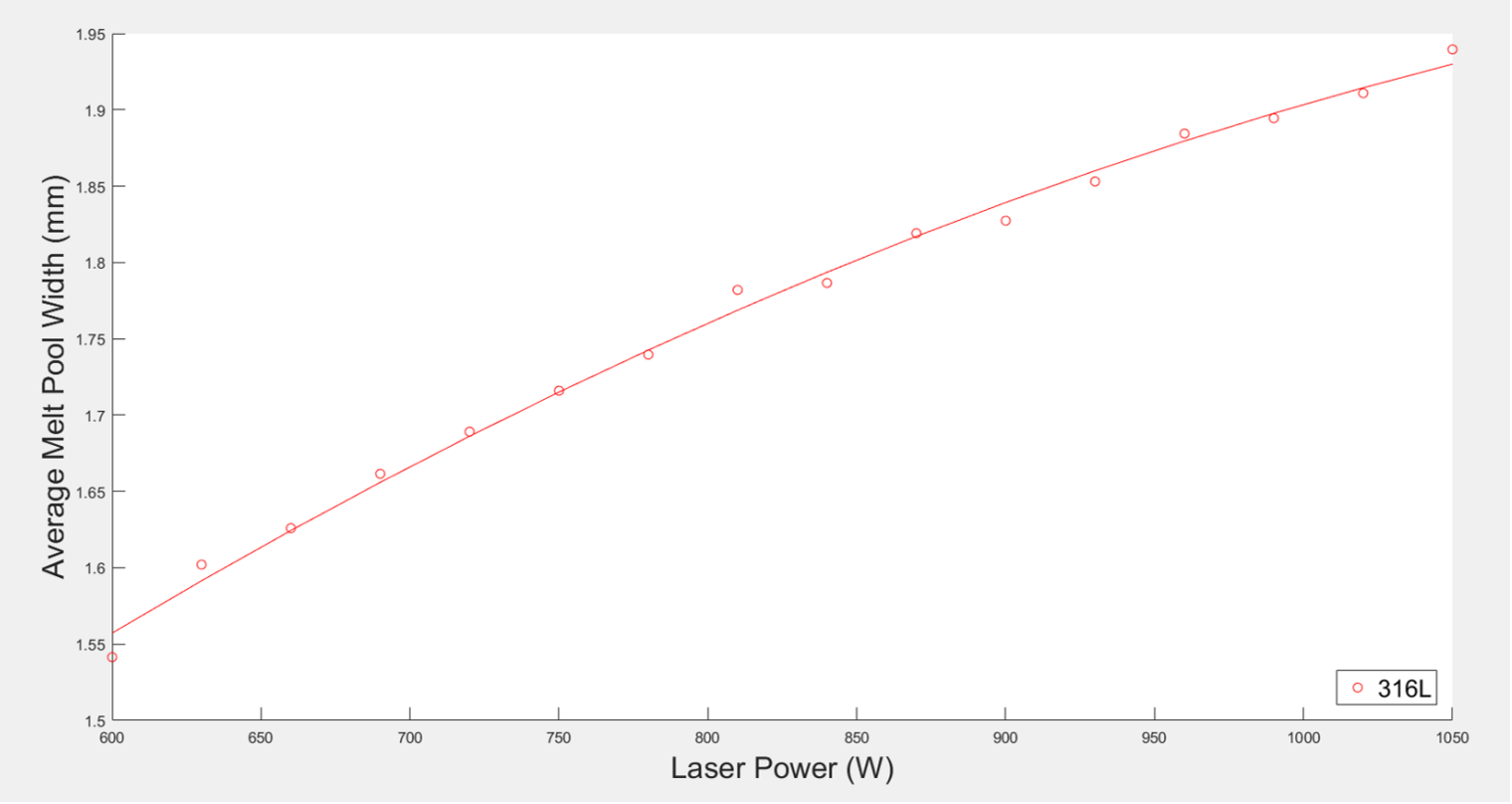

Figure 65: Average melt pool readings over different laser power settings for the material 316L.

Figure 65 displays how the melt pool width changes for increasing laser power. The results from this graph are like those achieved when performing a similar experiment with the material EN25. Increasing the laser power results in an increased melt pool width. This is expected as higher laser power settings result in a higher energy density, subsequently resulting in more powder being able to be melted. The line of best fit used for this graph is a second order polynomial. The second order polynomial fits well to these points with little to no deviation in any of the readings. The gradient of the first portion of the graph is greater than the gradient in the latter. This relationship is thought to be due to the limiting factors changing as the laser power increases. When there is an abundance of powder in the melt pool, increasing laser power will have a more drastic effect on melt pool size. When there is an abundance of laser power in the melt pool situation, the melt pool size becomes limited 
due to a lack of powder.

The average melt pool size over different laser power settings for the material $316 \mathrm{~L}$ was then directly compared to the results achieved from performing the similar experiment on the material EN25. The results from these two experiments are plotted together in Figure 66.

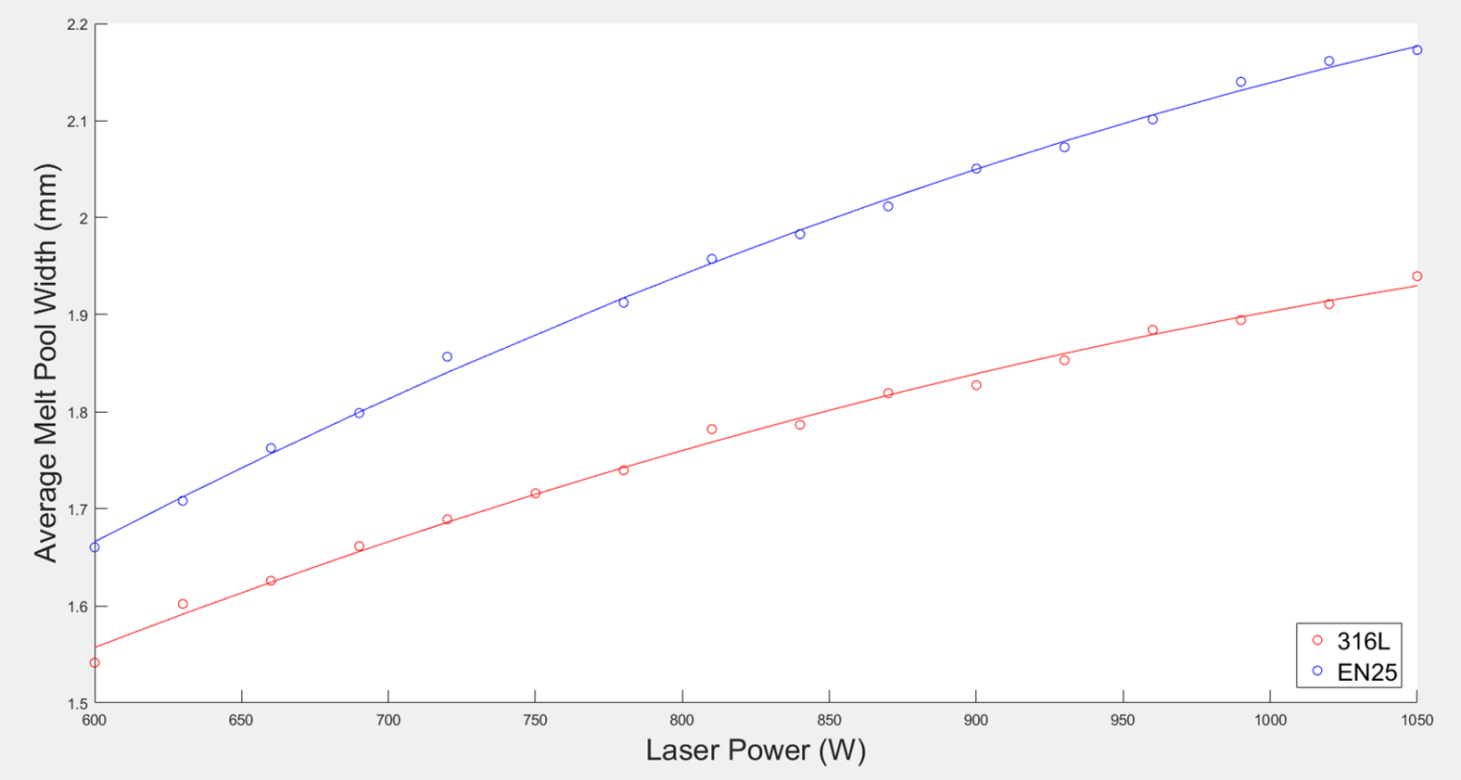

Figure 66: A graph to compare average melt pool widths for 316L and EN25.

Figure 66 directly compares the average melt pool width for 316L and EN25 deposition. It can be seen from this graph that both sets of data complement a second order polynomial line of best fit. Neither of the two sets of data points show much deviance from the line of best fit, and both show a very similar change in gradient when increasing the laser power setting. Depositing with EN25 produces a larger melt pool size using the same laser power settings when compared with the material 316L. This difference in melt pool size could be due to the differences in melting temperatures between the two materials. The difference in size between the EN25 and 316L deposition tracks increases with increasing laser power. EN25 produces a much larger melt pool at higher laser power settings.

Directly comparing these two graphs together can help industrial staff select optimal parameter 
settings for new materials. If the industrial technician has worked previously with one of these materials, and knows the optimal parameter settings for a specific component, then this analysis can be used to tailor existing settings for the new material. An example of this would be if the technician is fluent in producing components made from EN25. Looking at a Figure 66, the technician instantly knows that to achieve a melt pool size similar to the one used for EN25, the laser power must be reduced when using $316 \mathrm{~L}$.

\subsubsection{Conclusion}

Using new user defined values in the directional emittance algorithm has allowed for a new powder to be analysed and compared with EN25. Whilst results from analysing the videos of the higher laser power settings showed some incorrect melt pool widths, most frames in these videos produced accurate melt pool readings. Using the algorithm to produce melt pool widths on the lower laser power setting showed accurate and stable results. Average melt pool values were calculated, which subsequently allowed for the direct comparison of two DED powders, EN25 and 316L. Comparing how melt pool width changes with increasing laser power for these two materials has provided industrial staff with a method of being able to quickly compare new materials to reduce the parameter optimisation procedure.

\subsection{Discussion}

Currently there are no melt pool dimensioning techniques that can work independent of material properties. Conventional melt pool dimensioning techniques correlate pixel intensity values with temperature and perform a binary threshold to highlight melt pool edge features. New materials require the user to calibrate the melt pool monitoring system so that pixel intensity value can again be correlated with melt pool edges. This chapter has provided a method of being able to calculate melt pool width without the need for lengthy calibration procedures or the calculation of material dependent emissivity values. In doing so users can easily change between materials in an industrial environment and swiftly adapt parameters.

To study how the new directional emittance algorithm could be used on different materials, a parameter study similar to the one performed in Chapter 4 was conducted using 316L. The parametric 
study deposited single line tracks with various laser power settings to establish how melt pool width changes. The directional emittance algorithm was able to successfully measure the melt pool width for a range of laser power settings without the need for a lengthy calibration period. Simple changes to the exposure time setting and certain algorithm coefficients allowed the directional emittance algorithm to perform melt pool width calculations on the new material. This allowed for the direct comparison of two materials and for further conformation of how melt pool width varies with laser power alterations.

The conventional image processing techniques, that use binary thresholds, correlate pixel intensity values with the surface temperature of the melt pool to determine the edge $[10,24,27,30-32,34,43-$ $46,60,86,87]$. Correlating these two values together requires the calculation of an emissivity value, which is temperature, material and surface angle dependent. Multiple papers have cited inaccuracies in this technique and it is known that determining accurate emissivity values for molten metals is difficult $[39,40,47]$. For this reason emissivity-based techniques should perform calibration to improve accuracy for each material, which will likely result in complex and lengthy experimental procedures. This chapter has shown that the new directional emittance algorithm can be used on new materials by simply adapting the COMS exposure time setting and certain coefficients in the underlying algorithm, reducing the amount of effort required to perform measurements on new materials.

Although the new directional emittance algorithm was able to adapt to the new material, there was some user interaction required for the technique to work. Both the exposure time and some algorithm coefficients had to be altered, meaning that user interaction would be required for each new material used. This reduces the functionality of the directional emittance algorithm and results in a technique that still requires user calibration for new materials.

Future work should attempt to reduce the amount of user interaction required when using new materials and look to introduce a more autonomous system. The limitations highlighted in Chapter 3, concerning the lack of autonomous exposure time adaptation, have now had an effect on the algorithms performance with new materials. Introducing a method that can automatically select optimum exposure times to highlight the directional emittance features would reduce the amount of user calibration for new materials. This could potentially allow for the original coefficients in the directional emittance algorithm to be used for both materials without the need for change. If this is not feasible, 
a new technique in the algorithm should be created so that the coefficients can adapt automatically for different materials. The aim should be to develop a technique that can accurately measure melt pool width for all parameter and material selections.

Industrial environments witness regular changes in powder material and new materials are constantly being developed for AM processes. The complicated nature of emissivity calculations and changing materials provides a hurdle for the adoption of the conventional Canny edge technique into industrial environments. The applicability of the new directional emittance technique on multiple materials has been explored, and the technique was able to adapt to new materials with minor alterations. This technique shows promise in being able to perform melt pool measurements independent of material properties. This shows an improvement on the current state of the art melt pool monitoring techniques and provides a technique that can be more easily adopted into industrial environments.

\subsection{Chapter Conclusion}

The directional emittance algorithm developed has been successfully used to analyse different materials with very little calibration involved. The developed algorithm was introduced as an alternative to the originally designed Canny edge detection method, due to its prospects of being able to more accurately measure melt pool geometry, without the need for time consuming (and inaccurate) emissivity calculations.

The directional emittance algorithm initially struggled with the new data set, but it is concluded that this is down to the crude nature of the exposure time selection method developed in Chapter 3. This method of calculating exposure times for new laser power settings and materials has been incredibly valuable in the development of this research, but results from this chapter have highlighted the limitations. The original technique of calculating the exposure time based on the operator's decision has allowed for the development of the new algorithm, the implementation of this algorithm into a full working system, and the analysis of multiple DED materials. Whilst this technique has been used to analyse melt pool geometry with little calibration, in the process the user is responsible for both selecting the correct exposure time and user defined threshold values in the algorithm. This method has shown its limitation and subjective human decisions have led to more calibration procedures being 
required. The goal of this technique is for accurate melt pool measurements for all materials across all laser power settings. This goal remains and should be explored in future work.

Results gathered after minor alterations of the developed algorithm showed the potential of this technique. Accurate melt pool measurements have been acquired from recordings of DED using EN25 and $316 \mathrm{~L}$ powders. Average melt pool widths have been calculated for a range of different laser power settings to directly compare the size of the melt pool when manufacturing in different materials. This technique, although there are some minor flaws, has shown that it can be used in a variety of industrial projects to better understand material characteristics and optimise parameter settings. 


\section{Conclusion}

The aim of this research was to improve DED processes. Improving DED processes allows for higher quality manufacturing. This work has been successful in providing a system that is capable of more accurate melt pool measurements. This has subsequently led to a better understanding of the complex interaction between parameters throughout DED processes.

A major contribution to the success of the newly developed melt pool monitoring system is the understanding of the importance of exposure time. Previously documented studies have not focused on optimising melt pool imagery and have often overlooked the importance of tailoring the exposure time to specific melt pool scenarios. Changing the exposure time setting on the machine vision camera led to a reduction in the number of saturated pixels in melt pool images. Using a notch filter and reducing the amount of radiation that is emitted in the direction of the camera's sensor allowed for higher quality melt pool imaging. Higher quality images of the melt pool allowed for a more in-depth understanding of melt pool radiation mechanics and exposed details of the melt pool that have not yet been documented.

The window of exposure times that produce high quality melt pool images is small and dependant on the laser power setting. Increasing the laser power for fixed exposure times results in higher levels of thermal radiation being recoded with machine vision cameras. Increasing the laser power setting led to an increase in saturation levels in melt pool images and resulted in non-optimised imagery. An equation was calculated that adapted the exposure time for a given laser power setting to allow for high quality melt pool imagery at all laser power settings.

Improvements in melt pool imagery led to the discovery of the directional emittance phenomena. The directional emittance phenomena occurs in melt pool imagery as the underlying substrate emits more radiation in the direction of the camera's sensor than parts of the melt pool. Due to high surface angles at the melt pool edge, low levels of radiation are emitted in the direction of the cameras sensor. Due to the low angles of the substrate, radiation from the substrate is emitted at higher concentrations in the direction of the cameras sensor even though the surface temperature is lower. The directional emittance phenomena highlights the edge of the melt pool. This feature is used to calculate the melt 
pool width using a newly developed image processing algorithm.

The newly designed directional emittance algorithm was able to calculate melt pool width without the need for material-based emissivity values. The algorithm used techniques including frame averaging, signal extraction and differentiation to further accentuate the directional emittance phenomena. The new directional emittance algorithm was compared with the conventional Canny edge detection technique and it was discovered that the conventional Canny edge detection technique provided poor measurements. The conventional technique that used emissivity values and a single binary threshold required the edges of the melt pool to radiate at the same level of intensity in the direction of the cameras sensor. It was discovered that different points on the melt pool edge radiate at different intensities due to varying surface angles when attempting to use the Canny edge technique on improved melt pool images. The new directional emittance image processing technique provided more accurate and stable melt pool readings.

The directional emittance algorithm was used to better understand the complex DED process. A series of parametric studies were conducted, and the influence that laser power, translation speed and PMFR settings had on melt pool width was documented. Increasing the laser power setting resulted in an increase in the melt pool width, and the results indicated that this relationship can be approximated as a second order polynomial. Increasing the PMFR was originally thought to increase the melt pool width, but the improved algorithm uncovered a more detailed relationship. Increasing the PMFR at high laser power settings led to an increase in melt pool width, increasing PMFR setting at a low laser power setting resulted in no increase in melt pool width. Increasing the path velocity resulted in a decrease in melt pool width for all laser power settings. The rate that path velocity changed melt pool width was different for different laser power settings, but no further conclusion was drawn.

Further studies using the directional emittance algorithm allowed for the direct comparison between two different materials with little calibration. The new technique does not require the use of emissivity values and therefore can work relatively freely on different materials. Minor calibration procedures allowed for the comparison of melt pool widths for two different materials. Both materials showed a similar relationship to increasing laser power. Both materials fitted well to the second order polynomial line of best fit. 
Advances made in both optical set-up and image processing algorithms were encapsulated together into a single application. An application was developed for both academic and industrial purposes that allowed users to operate optical equipment, perform live melt pool width calculations, and record calculated measurements. The directional emittance image processing algorithm was optimised using GPGPU techniques. This resulted in a performance increase. This work is documented in the Appendix.

All aims that were originally defined in the introduction were achieved. This research has advanced melt pool monitoring systems in three ways: by optimising machine vision techniques, developing an improved image processing algorithm, and optimising the algorithm to be used in industrial environments. These advancements have been successfully used for parametric studies. Although much progress has been made in different research areas surrounding melt pool monitoring, more progress should be made to further advance the melt pool monitoring system. Chapter 8 encapsulates future work ideas regarding melt pool dimensioning, explaining how it can be used to further advance the capabilities of DED processes. 


\section{Future Work}

The concluding remarks from Chapter 7 identified that the new melt pool monitoring system has successfully improved the in-house DED systems at TWI. The new system can provide more information about parameters and materials, as well as providing an application that can allow industrial staff to monitor melt pool dimensions in real time during AM build cycles (detailed in the Appendix). Though these advancements have been helpful in developing DED processes, the real benefits and validity of introducing melt pool monitoring systems into DED systems (and potentially AM in general) currently reside in the future prospects. The use of melt pool monitoring systems to further advance AM capabilities is something that will be discussed in this chapter. After a broader discussion about the slightly distant future of melt pool monitoring systems, a more detailed analysis of the limitations of the current system will be made. Future recommendations for immediate advancements of this system will then be discussed.

The use of melt pool monitoring in AM processes is just one piece of the larger puzzle. The overall aim for nearly all researchers in the field of AM is to improve part quality, reduce defects and increase the amount of control during deposition. The introduction of AM as a technology has granted users more design freedom, which is one of the key reasons for the rapid expansion of the technology. Whilst AM offers huge advantages over conventional manufacturing, the technology is still not without limitations. Certain AM components are not used in some industrial sectors due to the technology's poor repeatability. AM can produce some very high quality components, but when compared with other technologies that can produce single crystal turbine blades it is sometimes overshadowed.

The future of AM should aim to be faster and more robust. Future AM machinery should be able to produce specific microstructures and embed circuits seamlessly into components. AM components should be able to build in multiple materials, and not just similar materials. Builds should be able to contain circuits, metals, ceramics, plastics and smart materials. AM builds should have increased efficiency and less material should be wasted. AM builds should be able to produce components with minimal defects in a repeatable fashion so that they can be used in high end industrial applications.

Advancing AM to perform all these functionalities is a mammoth task for a single researcher. 
To perform such complex builds using AM systems, the system must be incredibly sophisticated and be able to control parameters to a very high precision. Even if a machine can do that, what parameters are required to merge two materials together? What parameters are required to produce a fine microstructure for EN25? or 316L? What parameters should be selected to stop certain materials cracking or having other defects? These are questions that require further research, which is beyond the scope of this work. This area of research is challenging due to the complex interactions between parameters throughout build cycles. The proposed solution to these questions is a detailed parameter monitoring system for further experimental studies.

\subsection{Detailed Parameter Monitoring System}

Melt pool monitoring in DED processes has proven to be useful, but the technique is not capable of providing answers to complex DED questions. The melt pool monitoring system should be accompanied by various other parameter monitoring techniques to provide a more conclusive analysis of exactly how the DED process is executing. The melt pool is important because it is the first instance in the DED process where major parameters interact with each other. It can provide information about build quality and defect formations with the introduction of new image processing algorithms. Melt pool monitoring can be used to monitor the changes of melt pool geometry when new layers are introduced, when heat accumulation happens, when manufacturing different geometries or when transitioning through acceleration/deceleration periods.

Melt pool monitoring cannot fully monitor parameters of DED builds that happen both before and after the molten instance. The flow of powder into the melt pool is something that should be monitored accurately. The PMFR is set on the in-house system as merely a percentage which governs how open a gate in the hopper is. A higher percentage set on the machine results in the gate being opened further. The bigger the opening, the more powder is introduced into the melt pool. This would indicate that the setting on the PMFR would govern a constant flow of powder to the melt pool, but this is certainly not the case. Changes occur during powder delivery due to nozzle blockages and carrier gas fluctuations. Random powder distributions also occur and cause fluctuations in PMFR delivery to the melt pool. These fluctuations can all effect melt pool stability and defects could form as a result. 
Research should link accurate PMFR values to melt pool features. The modular system created in the Appendix was created to allow for the expansion of a full parameter monitoring system. Research should investigate creating an optimised PMFR monitoring system to be integrated in to the existing melt pool monitoring software to improve in-situ parameter monitoring capabilities. Variations in PMFR and their effects on both melt pool geometry and clad quality should be studied.

Melt pool monitoring cannot measure events that occur after the instance captured on the CMOS sensor. Events such as cracking can occur during the melt pool solidification period, and studies have shown that defects can be detected by measuring the transient cool down phase [60]. The aim to control microstructure could also be feasible by introducing a method of measuring the solidification period in detail. Microstructure is well known to be influenced by the solidification period and there could be a potential link in the future between microstructure and measured cooling rates. The introduction of a thermal imaging camera to the currently developed software could open these two research avenues to explore the detection of defects and the design of microstructure. The research techniques of optimising optical hardware, designing algorithms and implementing them into industrial applications used in melt pool research can be adapted to work on trailing thermal imagery. Future research should optimise thermal imaging techniques and use them to study the behaviour of the solidification period. If successful, this research should be implemented into the industrial system developed in the Appendix. Further research should attempt to link melt pool geometry, or changes in melt pool geometry with resultant microstructures and defects.

By individually progressing these techniques and combining them together, a detailed parameter monitoring system can be developed. The newly developed parameter monitoring system will be capable of monitoring the PMFR, melt pool geometry, solidification period, cooling rates and defects formed. Monitoring these individual parameters can provide details of the DED process, but the ability to simultaneously monitor all of them will allow for deep analysis. Indications from the PMFR detector, melt pool monitoring system and thermal imaging cameras can be used together to provide location and likelihood of defects in the solidified material.

This system should be initially used for educational purposes to better understand the complex DED process. This will allow academics to understand exactly what causes instability in DED processes 
and how different parameters effect the melt pool, solidification stage and microstructure. Once this understanding is established, the system can be used in DED builds to produce optimised components and monitor their quality in-situ. This will allow for greater uptake of DED components. Control systems can then be implemented to maintain or adjust process parameters throughout build cycles to further optimise components. The key point in the development of this system is the constant communication between academic and industrial staff.

\subsection{Future Work of Melt Pool Monitoring Systems}

So far this chapter has given a detailed explanation of how to integrate melt pool monitoring into larger systems, but future work on the melt pool monitoring system developed is yet to be defined. Chapter 7 has documented that the new melt pool monitoring system has successfully improved TWI's in-house DED systems and due to this success research should be continued. The new directional emittance techniques developed have shown huge potential, by producing more accurate readings of the melt pool without the need for material dependent emissivity values. Future work should improve upon these advancements.

Calibration requirements for new materials or laser powers should be reduced by optimising the method of calculating exposure times. Chapter 6 highlighted limitations in the newly developed technique and showed that minor algorithm adjustments are required for dimensioning new materials. Research showed that the algorithm can adapt to new materials, but this requires user input. Research should focus on developing a new technique to automatically adapt exposure time. The new method should use an image processing technique to make the directional emittance phenomena maximum by adjusting the exposure time of the camera. Allowing the user to select an exposure time has led to incorrect measurements when using different materials. The new method should be able to automatically select different exposure times for all laser power settings and materials to allow for easy use in industrial environments. The selected exposure time should create the largest possible directional emittance phenomena for the easiest possible detection.

Another way to increase the image quality of the melt pool is by enhancing the optics. The camera selected for the melt pool algorithm development was installed coaxially at a fixed position on the laser 
deposition head. Focusing the camera on the laser deposition head was done using an iterative process by performing multiple deposition tracks and observing image quality. This required multiple deposition runs and minute adjustments of the cameras offset. Focus issues were also witnessed throughout build cycles. When depositing material, the substrate can warp, and non-optimal parameters can cause both overbuilds and underbuilds. When this occurs, images of the melt pool become unfocused, which could lead to incorrect measurements obtained from the image processing algorithms. The DOF should be increased to allow for focused melt pool images at a larger range of offsets. The internal optics should also be optimised so that the FOV covers more of the sensor to increase melt pool resolution. The tolerance envelopes of the new algorithm should be measured, and the optimisation of optical hardware should look to study and improve performance capabilities.

With improved images of the melt pool, more algorithms should be designed to further analyse the complex interaction of parameters and melt pool features. Other features of the melt pool such as dark/bright spots, melt pool length and aspect ratio should be studied further. Further work should study the optimised melt pool images in more detail and extract more features that can be used for control systems and deposition optimisation. The effect that parameters have on other melt pool features should be explored. 


\section{Glossary}

Additive Manufacturing - The process of joining materials to make objects from 3D model data, usually layer upon layer, as opposed to subtractive manufacturing methodologies.

Directional Emittance - The phenomena that radiation emitting from a surface is not equal in all directions for grey bodies.

Infrared Radiation - A type of electromagnetic radiation whose wavelengths range from around 700 nanometers to 1 millimeter.

Machine Vision - a technology and method usually used in industry to provide imaging-based automatic inspection and analysis for a variety of applications.

Near-Infrared Radiation - A type of electromagnetic radiation whose wavelengths range from around 700 nanometers to 1.4 micrometers.

Parallel Programming - A computational method by which many calculations are performed simultaneously.

Thermal Radiation - Electromagnetic radiation that is emitted from a body due to it possessing heat.

Thermography - A test that uses infrared cameras to detect heat emitting from a given object or surface. 


\section{Bibliography}

[1] T. Wohlers and T. Caffery, "Wohlers Report," Wohlers Associates, Fort Collins, Colorado, United States, Tech. Rep., 2015. [Online]. Available: https://wohlersassociates.com/

[2] S. Nathan, "Rolls-Royce breaks additive record with printed Trent-XWB bearing," The Engineer, jun 2015. [Online]. Available: https://www.theengineer.co.uk/issues/june-2015-digi-issue/ rolls-royce-breaks-additive-record-with-printed-trent-xwb-bearing/

[3] T. G. Spears and S. A. Gold, "In-process sensing in selective laser melting (SLM) additive manufacturing," Integrating Materials and Manufacturing Innovation, vol. 5, no. 2, pp. 2-25, 2016. [Online]. Available: http://link.springer.com/10.1186/s40192-016-0045-4

[4] C. Hull, "APPARATUS FOR PRODUCTION OF THREE-DIMENSIONAL OBJECTS BY STEREOLITHOGRAPHY," pp. 1-16, 1986. [Online]. Available: https://patents.google.com/ patent/US4575330A/en

[5] British Standard Institute, "Additive Manufacturing - General Principles - Terminology (BS ISO/ASTM 52900:2015)," British Standards, Tech. Rep., 2015.

[6] D. Locke and A. Candel-Ruiz, "Laser metal deposition defined," Industril Laser Solutions, nov 2010. [Online]. Available: https://www.industrial-lasers.com/articles/print/volume-250/issue-6/ features/laser-metal-deposition.html

[7] L. Technology, "LPW Technology," 2016. [Online]. Available: https://www.lpwtechnology.com/ technical-library/

[8] J. M. Pearce, "Appropedia," 2009. [Online]. Available: http://www.appropedia.org/Laser\{-\} Cladding

[9] D. Miller, E. Morris, and G. Colvin, "Defense Technical Information Center," pp. 38-42, 2016. [Online]. Available: http://www.dtic.mil/docs/citations/AD1029454 
[10] T. Craeghs, S. Clijsters, E. Yasa, and J.-P. Kruth, "Online quality control of selective laser melting," Solid Freeform Fabrication Symposium Proceedings, pp. 212-226, 2011. [Online]. Available: http://utwired.engr.utexas.edu/lff/symposium/proceedingsarchive/pubs/ Manuscripts/2011/2011-17-Craeghs.pdf

[11] A. Staff, "Composites Manufacturing," 2014. [Online]. Available: http:// compositesmanufacturingmagazine.com/2014/10/pros-cons-additive-manufacturing/

[12] S. L. N. Ford, "Additive Manufacturing Technology : Potential Implications for U . S . Manufacturing Competitiveness," Journal of International Commerce and Economics (USA), vol. 6, no. September, pp. 1-35, 2014.

[13] D. Salehi, M. Brandt, and R. Nagarajah, "Optical Monitoring of Nd : YAG Laser Produced Hastelloy C Clad Layers," Profiles in Industrial Research - Knowledge and Innovation 2002, vol. 1, no. 1, pp. 171-180, 2002. [Online]. Available: https://www.researchgate.net/publication/237682249\{-\} Optical \{-\}Monitoring $\left\{{ }_{-}\right\}$of $\left\{\left\{_{-}\right\}\right.$NdYAG $\left\{{ }_{-}\right\} \operatorname{Laser}\left\{\left\{_{-}\right\} \operatorname{Produced}\left\{_{-}\right\}\right.$Hastelloy $\left\{_{-}\right\} \mathrm{C}\left\{\left\{_{-}\right\} \mathrm{Clad}\left\{{ }_{-}\right\}\right.$Layers

[14] T. Hua, C. Jing, L. Xin, Z. Fengying, and H. Weidong, "Research on molten pool temperature in the process of laser rapid forming," Journal of Materials Processing Technology, vol. 198, no. 1-3, pp. $454-462,2008$.

[15] M. Tellez, "Fibre Laser Metal Deposition with Wire : Parameters Study and Temperature Control Thesis submitted to-the University of Nottingham," Ph.D. dissertation, The University of Nottingham, 2010. [Online]. Available: http://eprints.nottingham.ac.uk/12812/1/523510.pdf

[16] M. Pavlov, M. Doubenskaia, and I. Smurov, "Pyrometric analysis of thermal processes in SLM technology," Physics Procedia, vol. 5, pp. 523-531, 2010. [Online]. Available: http://dx.doi.org/10.1016/j.phpro.2010.08.080

[17] M. Islam, T. Purtonen, H. Piili, A. Salminen, and O. Nyrhilä, "Temperature profile and imaging analysis of laser additive manufacturing of stainless steel," Physics Procedia, vol. 41, no. 1, pp. 835-842, 2013. [Online]. Available: http://dx.doi.org/10.1016/j.phpro.2013.03.156 
[18] T. Furumoto, T. Ueda, M. R. Alkahari, and A. Hosokawa, "Investigation of laser consolidation process for metal powder by two-color pyrometer and high-speed video camera," CIRP Annals - Manufacturing Technology, vol. 62, no. 1, pp. 223-226, 2013. [Online]. Available: http://dx.doi.org/10.1016/j.cirp.2013.03.032

[19] A. R. Nassar, J. S. Keist, E. W. Reutzel, and T. J. Spurgeon, "Intra-layer closed-loop control of build plan during directed energy additive manufacturing of Ti-6Al-4V," Additive Manufacturing, vol. 6, no. 1, pp. 39-52, 2015. [Online]. Available: http://dx.doi.org/10.1016/j.addma.2015.03.005

[20] T. Furumoto, M. R. Alkahari, T. Ueda, M. S. A. Aziz, and A. Hosokawa, "Monitoring of Laser Consolidation Process of Metal Powder with High Speed Video Camera," Laser Assisted Net shape Engineering 7 (LANE 2012), vol. 39, no. 0, pp. 760-766, 2012. [Online]. Available: http://www.sciencedirect.com/science/article/pii/S1875389212026259

[21] T. Furumoto, K. Egashira, K. Munekage, and S. Abe, "Experimental investigation of melt pool behaviour during selective laser melting by high speed imaging," CIRP Annals - Manufacturing Technology, vol. 67, no. 1, pp. 253-256, 2018. [Online]. Available: https://doi.org/10.1016/j.cirp.2018.04.097

[22] R. Monier, F. Thumerel, J. Chapuis, F. Soulié, R. Monier, F. Thumerel, J. Chapuis, F. Soulié, and C. Bordreuil, "Liquid metals surface temperature fields measurements with a two-colour pyrometer To cite this version : HAL Id : hal-01576867," Measurement, vol. 101, pp. 72-80, 2017. [Online]. Available: http://dx.doi.org/10.1016/j.measurement.2016.12.031

[23] F. Meriaudeau and F. Truchetet, "Control and optimization of the laser cladding process using matrix cameras and image processing," Journal of Laser Applications, vol. 8, no. 6, p. 317, 1996.

[24] F. M. F. T. D. Grevey and a. B. Vannes, "Laser Cladding process and image processing," Journal of laser in engineering, vol. 6, no. 1, pp. 161-187, 1997. [Online]. Available: http://www.le2i.cnrs.fr/IMG/publications/130\{_\}Lasersinengineering-97.pdf

[25] E. Renier, F. Meriaudeau, and F. Truchetet, "CCD Technology Applied To Industrial Welding Applications," Proceedings of 8th Mediterranean Electrotechnical Conference on Industrial Appli- 
cations in Power Systems, Computer Science and Telecommunications (MELECON 96), vol. 1, no. 1, pp. 1335-1338, 1996. [Online]. Available: https://ieexplore.ieee.org/document/551193

[26] P. Lott, H. Schleifenbaum, W. Meiners, K. Wissenbach, C. Hinke, and J. Bültmann, "Design of an optical system for the in situ process monitoring of Selective Laser Melting (SLM)," Physics Procedia, vol. 12, pp. 683-690, 2011. [Online]. Available: http://dx.doi.org/10.1016/j.phpro.2011.03.085

[27] D. Hu and R. Kovacevic, "Sensing, modelling and control for laser-based additive manufacturing," International Journal of Machine Tools and Manufacture, vol. 43, no. 1, pp. 51-60, 2003. [Online]. Available: https://www.sciencedirect.com/science/article/pii/S0890695502001633

[28] J. Kruth, P. Mercelis, J. V. Vaerenbergh, and T. Craeghs, "Feedback control of Selective Laser Melting," Procedings of the 15th International Symposium on Electromachining, vol. 1, no. 1, pp. 421-426, 2007. [Online]. Available: https://core.ac.uk/download/pdf/34389507.pdf

[29] S. Berumen, F. Bechmann, S. Lindner, J.-P. Kruth, and T. Craeghs, "Quality control of laserand powder bed-based Additive Manufacturing (AM) technologies," Physics Procedia, vol. 5, no. 1, pp. 617-622, 2010. [Online]. Available: https://www.sciencedirect.com/science/article/pii/ S1875389210005158

[30] T. Craeghs, F. Bechmann, S. Berumen, and J. P. Kruth, "Feedback control of Layerwise Laser Melting using optical sensors," Physics Procedia, vol. 5, pp. 505-514, 2010. [Online]. Available: http://dx.doi.org/10.1016/j.phpro.2010.08.078

[31] T. Craeghs, S. Clijsters, J.-P. Kruth, F. Bechmann, and M.-C. Ebert, "Detection of Process Failures in Layerwise Laser Melting with Optical Process Monitoring," Physics Procedia, vol. 39, no. 1, pp. 753-759, 2012. [Online]. Available: http://linkinghub.elsevier.com/retrieve/pii/ S1875389212026247

[32] S. Clijsters, T. Craeghs, S. Buls, K. Kempen, and J. P. Kruth, "In situ quality control of the selective laser melting process using a high-speed, real-time melt pool monitoring system," 
International Journal of Advanced Manufacturing Technology, vol. 75, no. 5-8, pp. 1089-1101, 2014. [Online]. Available: https://link.springer.com/article/10.1007/s00170-014-6214-8

[33] S. Buls, S. Clijsters, and J.-P. Kruth, "Homogenizing the melt pool intensity distribution in the SLM process through system identification and feedback control," Solid Freeform Fabrication Symposium Proceedings, pp. 6-11, 2014. [Online]. Available: http://sffsymposium.engr.utexas. edu/sites/default/files/2014-002-Buls.pdf

[34] M. Asselin, E. Toyserkani, M. Iravani-Tabrizipour, and A. Khajepour, "Development of trinocular CCD-based optical detector for real-time monitoring of laser cladding," Proceedings of the IEEE (Institute of Electrical and Electronics Engineers) International Conference on Mechatronics E Automation, Niagara Falls, Canada, pp. 1190-1196, 2005. [Online]. Available: http: //ieeexplore.ieee.org/xpls/abs\{_\}all.jsp?arnumber $=1626722$

[35] D. R. Tobergte and S. Curtis, "Elevated Region Area Measurement For Quantitative Analysis Of Laser Beam Melting Process Stability," Journal of Chemical Information and Modeling, vol. 53, no. 9, pp. 1689-1699, 2013.

[36] S. Kleszczynski, J. zur Jocobsmuhlen, and J. T. Sehrt, "Error Detection in Laser Beam Melting Systems by High Resolution Imaging," Solid Freeform Fabrication Symposium Proceedings, pp. 1-14, 2012. [Online]. Available: https://www.researchgate.net/profile/Stefan\{_\}Kleszczynski/

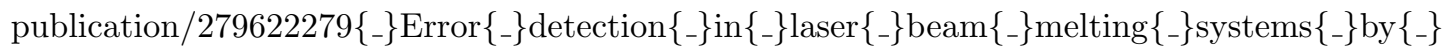
high \{_\}resolution \{_\}imaging/links/5742bf8b08ae298602ee540a.pdf

[37] L. Scime and J. Beuth, "Anomaly detection and classification in a laser powder bed additive manufacturing process using a trained computer vision algorithm," Additive Manufacturing, vol. 19, no. November 2017, pp. 114-126, 2018. [Online]. Available: http://dx.doi.org/10.1016/j.addma.2017.11.009

[38] — - "A multi-scale convolutional neural network for autonomous anomaly detection and classification in a laser powder bed fusion additive manufacturing process," Additive 
Manufacturing, vol. 24, no. October 2018, pp. 273-286, 2018. [Online]. Available: https://doi.org/10.1016/j.addma.2018.09.034

[39] E. Rodriguez, F. Medina, D. Espalin, C. Terrazas, D. Muse, C. Henry, E. Macdonald, R. B. Wicker, E. Paso, L. Martin, and A. Company, "Integration of a Thermal Imaging Feedback Control System in Electron Beam Melting," Solid Freeform Fabrication Symposium Proceedings, pp. 945-961, 2012. [Online]. Available: http://sffsymposium.engr.utexas.edu/Manuscripts/2012/ 2012-72-Rodriguez.pdf

[40] S. Ridwan, J. Mireles, S. Gaytan, D. Espalin, and R. Wicker, "Automatic Layerwise Acquisition of Thermal and Geometric Data of the Electron Beam Melting Process Using Infrared Thermography," Solid Freeform Fabrication Symposium Proceedings, pp. 343-352, 2014. [Online]. Available: http://sffsymposium.engr.utexas.edu/sites/default/files/2014-030-Ridwan.pdf

[41] J. Mireles, C. Terrazas, F. Medina, R. Wicker, and E. Paso, "Automatic Feedback Control in Electron Beam Melting Using Infrared Thermography," International Solid Freeform Fabrication Symposium An Additive Manufacturing Conference, pp. 708-717, 2013.

[42] J. Mireles, S. Ridwan, P. A. Morton, A. Hinojos, and R. B. Wicker, "Analysis and correction of defects within parts fabricated using powder bed fusion technology," Surface Topography: Metrology and Properties, vol. 3, no. August 2015, pp. 1-8, 2015. [Online]. Available: http://dx.doi.org/10.1088/2051-672X/3/3/034002

[43] P. Colodrón, J. Fariña, J. J. Rodríguez-Andina, F. Vidal, J. L. Mato, and M. Á. Montealegre, "FPGA-based measurement of melt pool size in laser cladding systems," Proceedings - ISIE 2011: 2011 IEEE (Institute of Electrical and Electronics Engineers) International Symposium on Industrial Electronics, vol. 1, no. 1, pp. 1503-1508, 2011. [Online]. Available: https: //ieeexplore.ieee.org/document/5984383

[44] P. Colodrón, J. Fariña, J. J. Rodríguez-Andina, F. Vidal, J. L. Mato, and Á. Montealegre, "Performance improvement of a laser cladding system through FPGA-based control," IECON 
Proceedings (Industrial Electronics Conference), vol. 1, no. 1, pp. 2814-2819, 2011. [Online]. Available: https://ieeexplore.ieee.org/document/6119758

[45] J. R. Araujo, J. J. Rodriguez-Andina, J. Farina, F. Vidal, J. L. Mato, and M. A. Montealegre, "FPGA-based laser cladding system with increased robustness to optical defects," IECON 2012 38th Annual Conference on IEEE Industrial Electronics Society, vol. 1, no. 1, pp. 4688-4693, 2012. [Online]. Available: http://ieeexplore.ieee.org/lpdocs/epic03/wrapper.htm?arnumber=6389491

[46] J. Hofman, B. Pathiraj, J. van Dijk, D. de Lange, and J. Meijer, "A camera based feedback control strategy for the laser cladding process," Journal of Materials Processing Technology, vol. 212, no. 11, pp. 2455-2462, 2012. [Online]. Available: http: //dx.doi.org/10.1016/j.jmatprotec.2012.06.027

[47] B. Cheng, J. Lydon, K. Cooper, V. Cole, P. Northrop, and K. Chou, "Melt pool sensing and size analysis in laser powder-bed metal additive manufacturing," Journal of Manufacturing Processes, vol. 32, no. November 2017, pp. 744-753, 2018. [Online]. Available: https://doi.org/10.1016/j.jmapro.2018.04.002

[48] D. Ye, K. Zhu, J. Ying, H. Fuh, Y. Zhang, and H. Geok, "The investigation of plume and spatter signatures on melted states in selective laser melting," Optics and Laser Technology, vol. 111, no. October 2018, pp. 395-406, 2019. [Online]. Available: https://doi.org/10.1016/j.optlastec.2018.10.019

[49] Y. Zhang, J. Y. H. Fuh, D. Ye, and G. Soon, "In-situ monitoring of laser-based PBF via off-axis vision and image processing approaches," Additive Manufacturing, vol. 25, no. October 2018, pp. 263-274, 2019. [Online]. Available: https://doi.org/10.1016/j.addma.2018.10.020

[50] S. Coeck, M. Bisht, J. Plas, and F. Verbist, "Prediction of lack of fusion porosity in selective laser melting based on melt pool monitoring data," Additive Manufacturing, vol. 25, no. October 2018, pp. 347-356, 2019. [Online]. Available: https://doi.org/10.1016/j.addma.2018.11.015

[51] M. Khanzadeh, W. Tian, A. Yadollahi, H. R. Doude, and M. A. Tschopp, "Dual process monitoring of metal-based additive manufacturing using tensor decomposition of thermal image 
streams," Additive Manufacturing, vol. 23, no. August 2018, pp. 443-456, 2018. [Online]. Available: https://doi.org/10.1016/j.addma.2018.08.014

[52] L. Scime and J. Beuth, "Using machine learning to identify in-situ melt pool signatures indicative of flaw formation in a laser powder bed fusion additive manufacturing process," Additive Manufacturing, vol. 25, no. October 2018, pp. 151-165, 2019. [Online]. Available: https://doi.org/10.1016/j.addma.2018.11.010

[53] J. L. Bartlett, F. M. Heim, Y. V. Murty, and X. Li, "In situ defect detection in selective laser melting via full- field infrared thermography," Additive Manufacturing, vol. 24, no. July, pp. 595-605, 2018. [Online]. Available: https://doi.org/10.1016/j.addma.2018.10.045

[54] G. Repossini, V. Laguzza, M. Grasso, and B. M. Colosimo, "On the use of spatter signature for in-situ monitoring of Laser Powder Bed Fusion," Additive Manufacturing, vol. 16, pp. 35-48, 2017. [Online]. Available: http://dx.doi.org/10.1016/j.addma.2017.05.004

[55] M. Bisht, N. Ray, F. Verbist, and S. Coeck, "Correlation of selective laser melting-melt pool events with the tensile properties of Ti-6Al-4V ELI processed by laser powder bed fusion," Additive Manufacturing, vol. 22, no. May 2018, pp. 302-306, 2018. [Online]. Available: https://doi.org/10.1016/j.addma.2018.05.004

[56] T. Kolb, A. Mahr, F. Huber, J. Tremel, and M. Schmidt, "Qualification of channels produced by laser powder bed fusion : Analysis of cleaning methods, flow rate and melt pool monitoring data," Additive Manufacturing, vol. 25, no. November 2018, pp. 430-436, 2019.

[57] D. Ye, J. Ying, H. Fuh, Y. Zhang, G. Soon, and K. Zhu, "In situ monitoring of selective laser melting using plume and spatter signatures by deep belief networks," ISA Transactions, vol. 81, no. July 2018, pp. 96-104, 2018. [Online]. Available: https://doi.org/10.1016/j.isatra.2018.07.021

[58] Y. Zhang, G. Soon, D. Ye, K. Zhu, and J. Y. H. Fuh, "Extraction and evaluation of melt pool, plume and spatter information for powder-bed fusion AM process monitoring," Materials \& Design, vol. 156, no. 1, pp. 458-469, 2018. [Online]. Available: https://doi.org/10.1016/j.matdes.2018.07.002 
[59] P. A. Hooper, "Melt pool temperature and cooling rates in laser powder bed fusion," Additive Manufacturing, vol. 22, no. May 2018, pp. 548-559, 2018. [Online]. Available: https://doi.org/10.1016/j.addma.2018.05.032

[60] J. Zalameda, E. Burke, and R. Hafley, "Thermal Imaging for Assessment of Electron-Beam Free Form Fabrication (EBF3) Additive Manufacturing Welds," Schematic Scholar, 2013. [Online]. Available: https://www.semanticscholar. org/paper/Thermal-Imaging-for-Assessment-of-Electron-Beam-(-3-Zalameda-Burkea/ a2d4f55242c049f9eb24308ec14f851c5d7822ae

[61] M. H. Farshidianfar, A. Khajepour, and A. Gerlich, "Real-time control of microstructure in laser additive manufacturing," International Journal of Advanced Manufacturing Technology, vol. 82, no. July 2015, pp. 1-15, 2016.

[62] C. Laser, "Concept Laser Quality Management Systems," 2019. [Online]. Available: https://www.concept-laser.de/en/products/quality-management.html

[63] Concept Laser, "Achieve the highest possible quality in series production thanks to LaserCUSING," 2019. [Online]. Available: https://www.concept-laser.de/fileadmin/user\{-\} upload/1603\{_\}QM $\left\{{ }_{-}\right\}$Prospect $\left\{{ }_{-}\right\}$EN.pdf

[64] Q. Guo, C. Zhao, L. I. Escano, Z. Young, L. Xiong, K. Fezzaa, W. Everhart, B. Brown, T. Sun, and L. Chen, "Transient dynamics of powder spattering in laser powder bed fusion additive manufacturing process revealed by in-situ high-speed high- energy x-ray imaging," Acta Materialia, vol. 151, no. 1, pp. 169-180, 2018. [Online]. Available: https://doi.org/10.1016/j.actamat.2018.03.036

[65] J. A. Kanko, A. P. Sibley, and J. M. Fraser, "In situ morphology-based defect detection of selective laser melting through inline coherent imaging," Journal of Materials Processing Tech., vol. 231, pp. 488-500, 2016. [Online]. Available: http://dx.doi.org/10.1016/j.jmatprotec.2015.12.024

[66] A. Heralić, A.-K. Christiansson, and B. Lennartson, "Height control of laser metalwire deposition based on iterative learning control and 3D scanning," Optics and 
Lasers in Engineering, vol. 50, no. 9, pp. 1230-1241, 2012. [Online]. Available: http: //linkinghub.elsevier.com/retrieve/pii/S0143816612001017

[67] S. Karnati, N. Matta, T. Sparks, and F. Liou, "Vision-based process monitoring for Laser Metal deposition processes," 24th International Solid Freeform Fabrication Symposium - An Additive Manufacturing Conference, SFF 2013, August 12, 2013 - August 14, 2013, pp. 88-94, 2013.

[68] M. Abdelrahman and T. L. Starr, "Layerwise monitoring of polymer Laser Sintering using thermal imaging," Solid Freeform Fabrication Symposium Proceedings, pp. 244-255, 2014. [Online]. Available: http://sffsymposium.engr.utexas.edu/sites/default/files/2014-021-Abdelrahman.pdf

[69] T. Wang, J. Chen, X. Gao, and W. Li, "Quality Monitoring for Laser Welding Based on High-Speed Photography and Support Vector Machine," Applied Sciences, vol. 7, no. 3, p. 299, 2017. [Online]. Available: http://www.mdpi.com/2076-3417/7/3/299

[70] HowStuffWorks.com, "What is the difference between CCD and CMOS image sensors in a digital camera?" What is the difference between CCD and CMOS image sensors in a digital camera?, 2000. [Online]. Available: https://electronics.howstuffworks.com/cameras-photography/digital/ question362.htm $\{\#\}$

[71] Cannon, "Cannon Website," 2018. [Online]. Available: http://cpn.canon-europe.com/content/ education/infobank/capturing $\left\{{ }_{-}\right\}$the $\left\{{ }_{-}\right\}$image/ $\operatorname{ccd}\left\{{ }_{-}\right\}$and $\left\{{ }_{-}\right\} \operatorname{cmos}\{-\}$ sensors.do

[72] British Standard Institute, "Optics and photonics - Spectral bands (BS ISO 20473:2007)," British Standards, Tech. Rep., 2007. [Online]. Available: https://www.iso.org/standard/39482.html

[73] C. Meola, Infrared Thermography : Recent Advances And Future Trends., 1st ed., C. Meola, Ed. Naples, Italy: Bentham eBooks, 2016, no. 1. [Online]. Available: https://ebooks.benthamscience. com/book/9781608051434/

[74] IDS-Imaging, "IDS-Imaging," 2018. [Online]. Available: https://en.ids-imaging.com/store/ u3-3240ml.html 
[75] J. Flammer, M. Mozaffarieh, and H. Bebie, Basic Sciences in Ophthalmology. Berlin, Heielberg: Springer, 2013. [Online]. Available: http://link.springer.com/10.1007/978-3-642-32261-7

[76] M. Vollmer, K.-P. Möllmann, and J. A. Shaw, "The optics and physics of near infrared imaging," The Education and Training in Optics and Photonics Conference Proceedings, pp. 1-8, 2015. [Online]. Available: http://proceedings.spiedigitallibrary.org/proceeding.aspx?doi=10.1117/12. 2223094

[77] E. Optics, "Edmund Optics," 2018. [Online]. Available: https://www.edmundoptics.co.uk/ optics/optical-filters/imaging-filters/uv-vis-cut-off-m30.5-x-0.50-machine-vision-filter/

[78] L. Universitet, "Linkopings Universitet," 2014. [Online]. Available: http://www.cvl.isy.liu.se/ education/tutorials/rolling-shutter-tutorial/

[79] M. Gregson, "Cool Pumpkins Photography," 2014. [Online]. Available: http://www. coolpumpkins.com/Shutter\{_\}SPeed $\{$ \} Record $\{$ - $\}$ Action.html

[80] B. Cheng, J. Lydon, K. Cooper, V. Cole, P. Northrop, and K. Chou, "Melt Pool Dimension Measurement In Selective Laser Melting Using Thermal Imaging," Solid Freeform Fabrication Symposium Proceedings, pp. 1252-1263, 2017. [Online]. Available: http://sffsymposium.engr.utexas. edu/sites/default/files/2017/Manuscripts/MeltPoolDimensionMeasurementinSelectiveLaser.pdf

[81] S. Liu, W. Liu, M. Harooni, J. Ma, and R. Kovacevic, "Real-time monitoring of laser hot-wire cladding of Inconel 625," Optics and Laser Technology, vol. 62, pp. 124-134, 2014. [Online]. Available: http://dx.doi.org/10.1016/j.optlastec.2014.03.007

[82] Y. Zhang, G. Chen, C. Zhou, Y. Jiang, P. Zhong, and S. Li, "Pores formation in laser-MAG welding of 42CrMo steel," Journal of Materials Processing Technology, vol. 245, pp. 309-317, 2017. [Online]. Available: http://dx.doi.org/10.1016/j.jmatprotec.2017.02.029

[83] Trumpf, "Focusing Optics - Technical Data," 2019. [Online]. Available: https://www.trumpf. com/en \{-\}GB/products/laser/processing-optics/focusing-optics/ 
[84] Nikon, "Depth of Field and Depth of Focus," 2019. [Online]. Available: https: //www.microscopyu.com/microscopy-basics/depth-of-field-and-depth-of-focus

[85] Sciencing, "How to Calculate the Field of View in a Microscope," 2019. [Online]. Available: https://sciencing.com/calculate-field-microscope-7603588.html

[86] H. Krauss, C. Eschey, and M. Zaeh, "Thermography for Monitoring the Selective Laser Melting Process," Solid Freeform Fabrication Symposium Proceedings, pp. 999-1014, 2015. [Online]. Available: http://sffsymposium.engr.utexas.edu/Manuscripts/2012/2012-76-Krauss.pdf

[87] G. Kinsman and W. W. Duley, "METHOD AND APPARATUS FOR REAL-TIME CONTROL OF LASER PROCESSING OF MATERIALS," pp. 1-21, 1996. [Online]. Available: https://patents.google.com/patent/US5517420

[88] J. Canny, "A Computational Approach to Edge Detection," IEEE Transactions on Pattern Analysis and Machine Intelligence, vol. PAMI-8, no. 6, pp. 679-689, 1986. [Online]. Available: https://ieeexplore.ieee.org/document/4767851

[89] M. Mani, B. Lane, S. Feng, S. Feng, and S. Moylan, "Measurement Science Needs for Real-time Control of Additive Manufacturing Powder Bed Fusion Processes," National Institue of Standard and Technology, 2015.

[90] Y. Sun and M. Hao, "Statistical analysis and optimization of process parameters in Ti6Al4V laser cladding using Nd:YAG laser," Optics and Lasers in Engineering, vol. 50, no. 7, pp. 985-995, 2012. [Online]. Available: http://dx.doi.org/10.1016/j.optlaseng.2012.01.018

[91] S. Barua, F. Liou, J. Newkirk, T. Sparks, J. N. Todd, D. Olivier, S. Borros, and G. Reyes, "Vision-based defect detection in laser metal deposition process," Rapid Prototyping Journal Rapid Prototyping Journal, vol. 20, no. 1, pp. 77-85, 2014. [Online]. Available: http://dx.doi.org/ 10.1108/RPJ-04-2012-0036\{\%\}5Cnhttp://dx.doi.org/10.1108/RPJ-01-2013-0012\{\%\}5Cnhttp: //dx.doi.org/10.1108/13552540510573365\{\%\}5Cnhttp://dx.doi.org/10.1108/RPJ-01-2012-0002 
[92] M. Fowler, UML Distilled - A Brief Guide To The Standard Object Modeling Language, 3rd ed. Boston: Addison Wesley, 2006.

[93] Francesco Cervone, "Medium," 2017. [Online]. Available: https://medium.com/ @cervonefrancesco/model-view-presenter-android-guidelines-94970b430ddf

[94] Oracle, "Oracle," 2018. [Online]. Available: https://www.oracle.com/java/

[95] H. Schildt, C++ The Complete Reference, 2nd ed. New York: McGraw-Hill, 1995.

[96] OpenCV, "OpenCV," 2017. [Online]. Available: http://opencv.org/

[97] NVIDIA, "NVIDIA," 2017. [Online]. Available: http://www.nvidia.co.uk/

[98] H. Mujtaba, "WCCF Tech," NVIDIA's Discrete GPU Market Share Climbed To 72.8\% in Q3 2017, AMD's Share Dropped From 30\% To 27\% PC Gaming Demand Leads To Rise in AIB Shipments, 2017. [Online]. Available: https://wccftech.com/ nvidia-amd-discrete-gpu-market-share-report-q3-2017/

[99] M. Harris, S. Sengupta, and J. D. Owens, "Parallel Prefix Sum (Scan) with CUDA Mark," Tech. Rep., 2007. [Online]. Available: http://dl.acm.org/citation.cfm?id=1407436 


\section{A Appendix - System Architecture Design and Algorithm Op- timisation}

This thesis aimed to develop a practical solution to industrial problems in AM. The academic based work contained within has proven that the directional emittance method has an advantage over the Canny edge detection technique, but subsequent work should look to implement this into industrial environments. This appendix contains research surrounding the academic advancements and witnesses the development of algorithm acceleration and software housing.

\section{A.1 Practical Implications of Concept Systems}

Chapters 3 and 4 have detailed improved machine vision techniques and the directional emittance algorithm accordingly. Both chapters have shown positive results and have granted a better subjective understanding of the complex DED process. The aim of this research however extends to improve the capabilities of the in-house DED systems at TWI. The literature review concludes that there are several ways DED systems can be improved, and most systems monitoring parameters. This appendix uses research developments in Chapters 3 and 4 as a foundation to build a system that has advanced parameter monitoring capabilities. This new system will be subsequently used to better understand and improve DED processes and aid industrial staff in monitoring DED parameters in-situ.

This appendix details the software architecture design that is used to house the new melt pool monitoring algorithm. This appendix explores the use of Graphics Processing Units (GPUs) to accelerate the directional emittance algorithm. Finally, this appendix displays the newly developed melt pool monitoring desktop application.

\section{A.2 System Requirements}

Before designing a system, careful consideration needs to be taken of what is required. This section details important functions that the developed system employs as well as concepts that should be incorporated into future systems. The following sections combine fundamental academic knowledge 
with industrial experience to develop a system from academic concepts. The designed system should be used for further academic study of DED processes and look to develop a DED parameter monitoring system for use in an industrial environment.

\section{A.2.1 Parameter Monitoring}

The most fundamental requirement for the developed system is to be able to monitor parameters through the DED process. Choosing the correct parameters for a given application in the DED process requires a skilled operator. Monitoring parameter changes throughout build cycles has proven on multiple occasions to provide greater understanding of the DED process (Please refer to literature conclusion in Chapter 2). This has subsequently helped academics and operators improve DED build quality.

Melt pool dimensions are one of the most discussed parameters in AM processes, and Chapter 2 compiles multiple papers that have studied melt pool characteristics in detail. The designed system should have the primary function of incorporating the directional emittance algorithm developed in Chapter 4. The system should be able to calculate, record and display melt pool width and melt pool centre of mass values in real time. Even without the implementation of a closed feedback loop, monitoring the melt pool has proven to provide a better understanding of AM processes and to improve AM quality. Having a real time, in-situ melt pool monitoring system implemented on the in-house DED machines will allow for a greater understanding of how the melt pool geometry changes throughout DED build cycles.

\section{A.2.2 Control Feedback}

The implementation of a control feedback loop is out of the scope of this research. This research has aimed to better understand the DED process and improve upon existing melt pool monitoring techniques. Although it is not high priority at this point in time, the implementation of a control feedback loop in the future is something that should be considered in the original designing of the architecture.

Implementing a control feedback loop requires the feedback of a parameter (such as the melt pool 
width) to allow the system to take corrective action. Multiple AM control systems have tailored laser power to manipulate melt pool geometry. From the research experiments and extensive literature review, multiple parameters are known to effect the melt pool. The designed system should be used to better understand the complex relationship between parameters such as laser power, path velocity and PMFR, and their effects on melt pool geometry. The designed system should be able to alter melt pool geometry in an effective and efficient way by altering multiple parameters simultaneously.

\section{A.2.3 Defect Detection}

Previous literature has cited that fluctuations in melt pool geometry can be linked with manufacturing defects [33]. Detecting defects within DED builds is an incredibly useful tool when manufacturing, and reduces the amount of material that is wasted by continuing the build on components that would already fail post validation tests.

Defects take many forms in DED builds and include: lack of fusion, excessive dilution, porosity and geometric failures (such as thin wall collapses). Providing a method of detecting defects would be highly advantageous for a DED monitoring systems Advancements on these techniques include hybrid manufacturing (where the defects can be removed and material can be re-deposited) and advanced in-situ NDT validation (where defects are analysed by more advanced NDT techniques), the latter of which can be used to classify defects.

The implementation of these more advanced systems is something that is out of the scope of this research, but the future implementation of a defect detection software module should be considered in the original architecture design. Future systems should look towards detecting defects in-situ to minimise waste material.

\section{A.2.4 Portability}

The AM parameter monitoring system should be designed to be implemented on multiple machines with ease. In academia, research can often become too focused, with systems being able to only perform well in specific laboratory environments. Industrial environments need to be considered, as the end result is to implement the developed academic concepts into production facilities. The components of 
DED machines vary greatly, and the system created should be able to adapt to these changes. This requirement is more dependent on the hardware design rather than software design. The hardware design created in Chapter 3 was kept minimalistic for this reason.

\section{A.2.5 Adaptability}

The field of AM is changing constantly due to its infancy. New literature and concepts are constantly being published with a large portion of these ideas captured in the original literature review (Chapter 2). For this reason, the system to be designed should be able to rapidly adapt to the constant changes of AM techniques.

Not only should adaptability be considered for the entire architecture design, but also in the lower levels of user interface. Having a system that can adapt to new hardware changes and machine development is critical, but similarly the system should be able to adapt to different projects and builds too. The system designed should be used in academic and industrial environments so both can influence development. Having this ability will allow for research to be tailored to suit industrial requirements, and grant technical staff an insight into complex parameter interactions.

\section{A.2.6 Processing Speed}

For the system to display images of the melt pool smoothly, processing individual frames must be completed at a reasonable rate. The future development of a control systems will also require high frame rates. For this reason, a method of accelerating the designed algorithm is required. This method should provide an increase in speed and be adaptable for future changes in the designed parameter monitoring system. Processing speed is discussed in detail within this appendix, and is one of the modules that is to be developed in the original software design.

\section{A.2.7 Data Storage}

Data produced through experimentation using the designed system can be used for subsequent future advancements. Machine learning is an incredibly useful tool that requires large amounts of data to teach computers specific tasks. Machine learning uses algorithms that allow systems to learn from data, 
and then make predictions and decisions. The field of AM is suited to machine learning techniques due to the incredibly complex web of parameters and the huge amount of data that can be collected from AM builds. The interaction between these parameters is incredibly complex and is something that would be almost impossible for a human to understand and categorise into a set of equations. With large amounts of data, relationships between different parameters should be explored using the power of machine learning. For this reason, suitable data storage should be considered to allow for the future development of system capabilities.

Data for this research is currently stored in MATLAB where a data matrix is constructed. A MATLAB scripts executes the newly designed image processing technique on a series of input videos that are labelled in a specific manner to provide information about the AM input parameters. File names have the following format $\mathrm{LP}^{* * * *} \mathrm{EX}^{*} . * * * \mathrm{PV}^{* *} . * * \mathrm{PR}^{* *}$.**. avi. The data matrix stores parameter values for laser power, exposure time, path velocity and PMFR. It also stores measurements for all frames, extracted snippets and calculates melt pool averages for entire clad tracks.

Further development of the current data storage method should look to make data storage easier for the user. Users should be able to easily create, save and load data. The amount of data that has been processed in this research is small, and therefore easy to manage using the current technique. For this reason, it is not of high priority to improve upon the current format.

\section{A.3 System Architecture Design}

System architecture design provides a solid foundation for the development of a system. By correctly planning out the architecture, an overview of the system is created and often leads to more accurate and organised source code.

The requirements defined in Section A.2 give a solid criteria that should be met by the developed system. With the optical hardware already solidified, the next stage of system development should focus on how to tie the optical hardware and directional emittance algorithm to a Graphic User Interface (GUI). Doing this will allow both academic and industrial staff to experiment and manufacture builds using the designed software easily, without the need for understanding core image processing algorithms and data handling. To provide this service, custom software has been written that can 
control the optical hardware and perform image processing to display melt pool data to the user using a GUI.

There are a range of different software architecture patterns that can be used to design software, allowing for more organised coding. The following book categorises these software architecture patterns into five categories (Layered architecture, Event-driven architecture, Microkernel architecture, Microservices architecture pattern, Space-based architecture), each having their individual pros and cons [92]. From these five categories, the layered architecture was the most relevant system architecture pattern. This would allow long term scalability of the developed solution, where critical components (contained within their own layer) could be moved to dedicated hardware developed for that task.

\section{A.3.1 The Layered Architecture Model}

The layered architecture pattern is currently the most common architecture pattern used, and can often be referred to as "n-tier" or "multitier" architecture patterns. It is commonly used in most Java EE applications and known by most software architects, designers and developers.

The layered architecture pattern organises components into horizontal layers with each layer performing a specific role. The number of layers can vary depending on the complexity of the application being developed, with layers forming an abstraction around the work and functions that are within. This abstraction causes separation of concerns among components, resulting in layer-specific components that only deal with logic in that particular layer. This results in the advantages of simpler testing, development, maintenance and governing.

Individual layers within the layered architecture can either be closed or open. Closed layers require the programmer to pass through each individual layer in a system, to gather information that is needed on a different layer. Open layers can allow for quicker response times, but unfortunately can have negative consequences and break the concept of "layers of isolation". This concept means that changes within a specific layer have no (or little) subsequent effect on components within another layer. Interdependencies between components results in inevitable code changes, bug fixes and alterations becoming time consuming and costly. The division of logic into different layers allows users to more easily design and alter code within a specific layer without requiring knowledge of other layers. 
The referenced book encapsulating multiple software architecture patterns compare these architectures on agility, ease of deployment, testability, performance, scalability and ease of development [92]. The author scores the layered architecture model "low" for agility, deployment, performance and scalability, and "high" for the testability and development. The development of this parameter monitoring system is in its infancy, so testability and development are incredibly important at this stage. For these reasons the layered architecture model has been selected for software development.

\section{A.3.1.1 The MVP model}

The MVP model is an architecture pattern that separates the concerns of an applications domain, presentation and user input into specialised components [93], and has been selected as the most appropriate model for this development. There are several patterns commonly accepted under the name "MVP" that all have slight variations to one another. The MVP model is composed of three components that all deal with individual aspects of the program. The model refers to the data and business functionality of the application. This can be for example a method of extracting information from a database, or algorithms that perform calculations and define data. The view is an interface that displays data (originally defined by the model) and contains logic for GUI components. The amount of logic that is within the view varies between different MVP-based patterns but modern MVP patterns tend to reduce logic in the view as much as possible. The presenter collects data from the model and interacts with the view to display data to the user. The presenter also handles events called by the user, interacts with the model, and displays updated data to the user by changing the view.

\section{A.3.2 JavaFX}

Java is a popular object-orientated language among coders and can be used to effectively develop application programs [94]. JavaFX is an open source Java-based framework for developing rich client applications and is the successor to more primitive Java application programming interfaces (API's) such as Java Swing. JavaFX is written in Java, meaning it has access to Java features such as multithreading, generics and lambda expressions. It also has access to FXML, a XML-based scriptable mark-up language to define user interfaces declaratively. JavaFX has the built in capability to handle 
multimedia tasks such as portraying audio and video information. Due to the popularity, cross-platform support, high functionality and cost, it has been chosen to be used for the construction of the view, presenter, and majority of the model components in the system architecture design.

\section{A.3.3 $\mathrm{C}++$ and CUDA $/ \mathrm{C}++$}

One of the core requirements defined for the system was processing speed. To allow for a smooth application of the developed algorithms and future implementation of a closed control feedback loop, image processing speeds need to be fast. Increasing the image processing speed is a trend that has seen much research surrounding it and is documented frequently in Chapter 2. The original image processing algorithm was designed in MATLAB, but other coding languages should be considered when implementing this code into the new system architecture. CUDA/C ++ is a coding language that utilises GPU's for fast algorithm implementation. It is an extension of the $\mathrm{C}++$ language that has CUDA related functions to communicate from the $\mathrm{C}++$ environment (run on the $\mathrm{CPU}$ ) to the CUDA/C++ environment implemented on the GPU. General Purpose programming on Graphics Processing Unit (GPGPU programming) is a method of using GPU's and parallel programming to increase the performance of data processing. This concept is explored in more detail in Section A.4. The image processing algorithm designed in Chapter 4 will be designed in both $\mathrm{C}++$ and CUDA/C++ programming languages to distinguish what technique yields the best performance.

\section{A.3.4 Final Architecture Concept}

The following final architecture concept coalesces the MVP model, previously discussed programming languages, developed hardware (Chapter 3) and directional emittance algorithm (Chapter 4) to design a system concept for future development. The final architecture concept is displayed in Figure A.1. IRIS (Infrared Radiation Inspection System) is the chosen name for the program.

\section{A.3.4.1 IRIS.exe}

IRIS.exe is an application developed to demonstrate the capability of the research presented in this thesis, and is required to develop algorithmic concepts. This executable program encapsulates all 
features. Everything within the grey section is written within JavaFX and all its contents follow the MVP architecture pattern. Starting from the top of Figure A.1, the User Heads Up Display (HUD) displays all information and allows for user interaction. The HUD contains two modules (which are detailed later in this section) that the user can freely change between, all of which run simultaneously and independent from one another. All four of these components within the view are FXML files with no logical elements to them.

The logic for the FXML files is written in the presenter. The two FXML module components that are present within this design are in direct contact with presenter counterparts. The files within the presenter contain all the logic for interaction with their view counterparts. This logic includes code blocks that are executed when events are called by the user, and code blocks that change the GUI based on data changes.

In this design, only one model is present. This model runs on a separate thread and allows the collection of data from the image processing executable. In conjunction with this, the model also feeds data to the presenters to allow for updates in the GUI/HUD. 


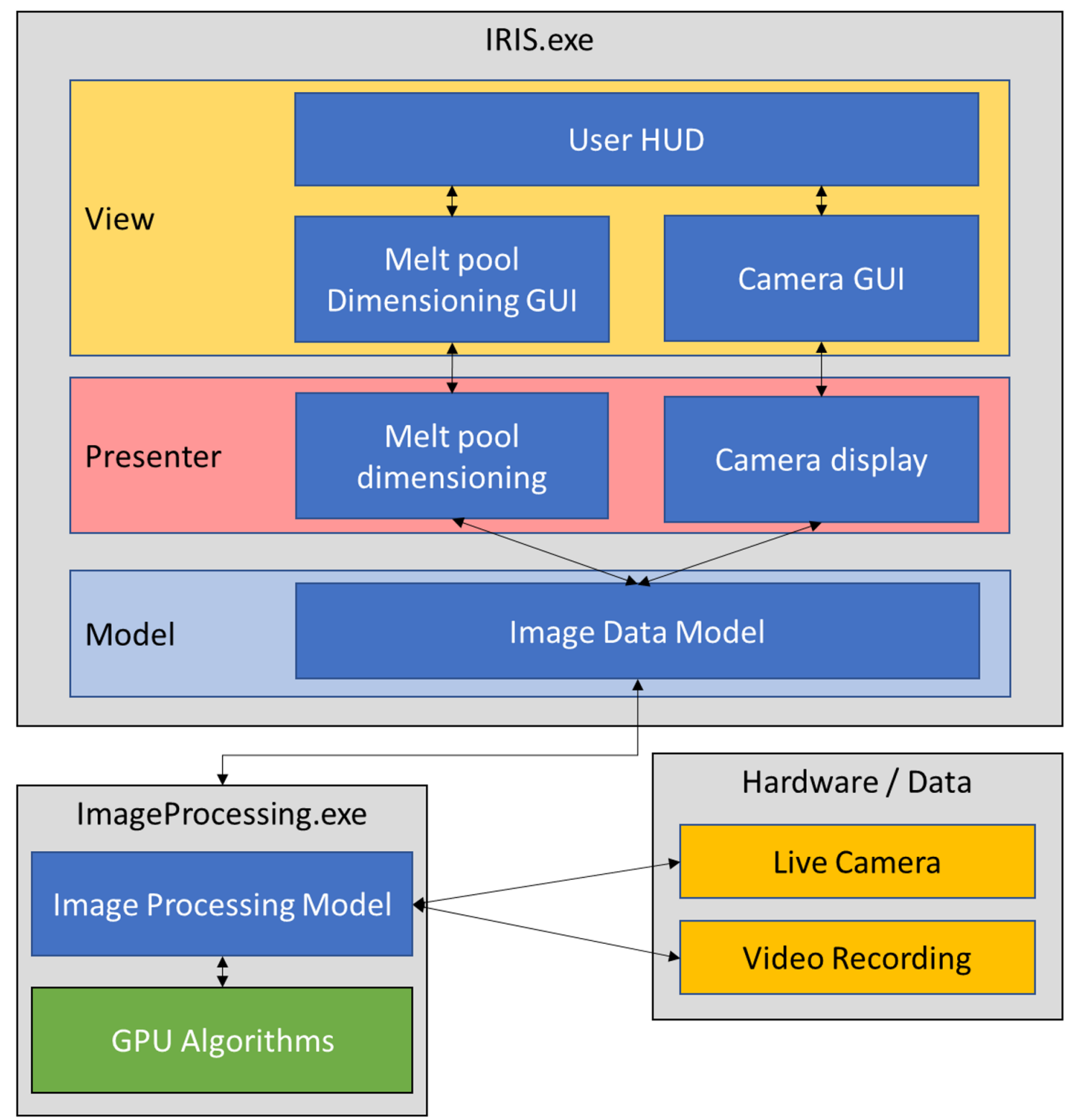

Figure A.1: A schematic diagram representing the architecture structure design to be used for further system development. 


\section{A.3.4.2 ImageProcessing.exe}

ImageProcessing.exe is an executable program that is written in CUDA/C ++ to take advantage of raw processing powder. The IRIS.exe application can launch the ImageProcessing.exe using its own thread to allow for constant data streaming through the standard input/output streams (this could be extended to a TCP stream in future work, where remote processing would be of benefit e.g. cloud based machine learning). The ImageProcessing executable can directly open and alter hardware/data, before manipulating it and implementing GPU algorithms on image feeds to extract melt pool dimensions. The ImageProcessing executable not only sends information to the Java model, but also receives commands given by the user. This design allows the user to be able to alter settings within the ImageProcessing executable from the high level User HUD.

\section{A.3.4.3 Future Design}

The system requirements promote adaptability to allow for the future incorporation of new hardware and modules. Section A.2.5 highlights the fast movement of AM technologies, and explains the importance of a developed system being able to adapt to these changes. The following system has been designed around this concept of adaptability, to allow for concepts in the system requirements to be added to subsequent system versions. The layered architecture design complements this concept, meaning that fluid expansion can occur to encapsulate future modules and/or hardware into the system. The diagram in Figure A.2 shows a future expansion concept. 


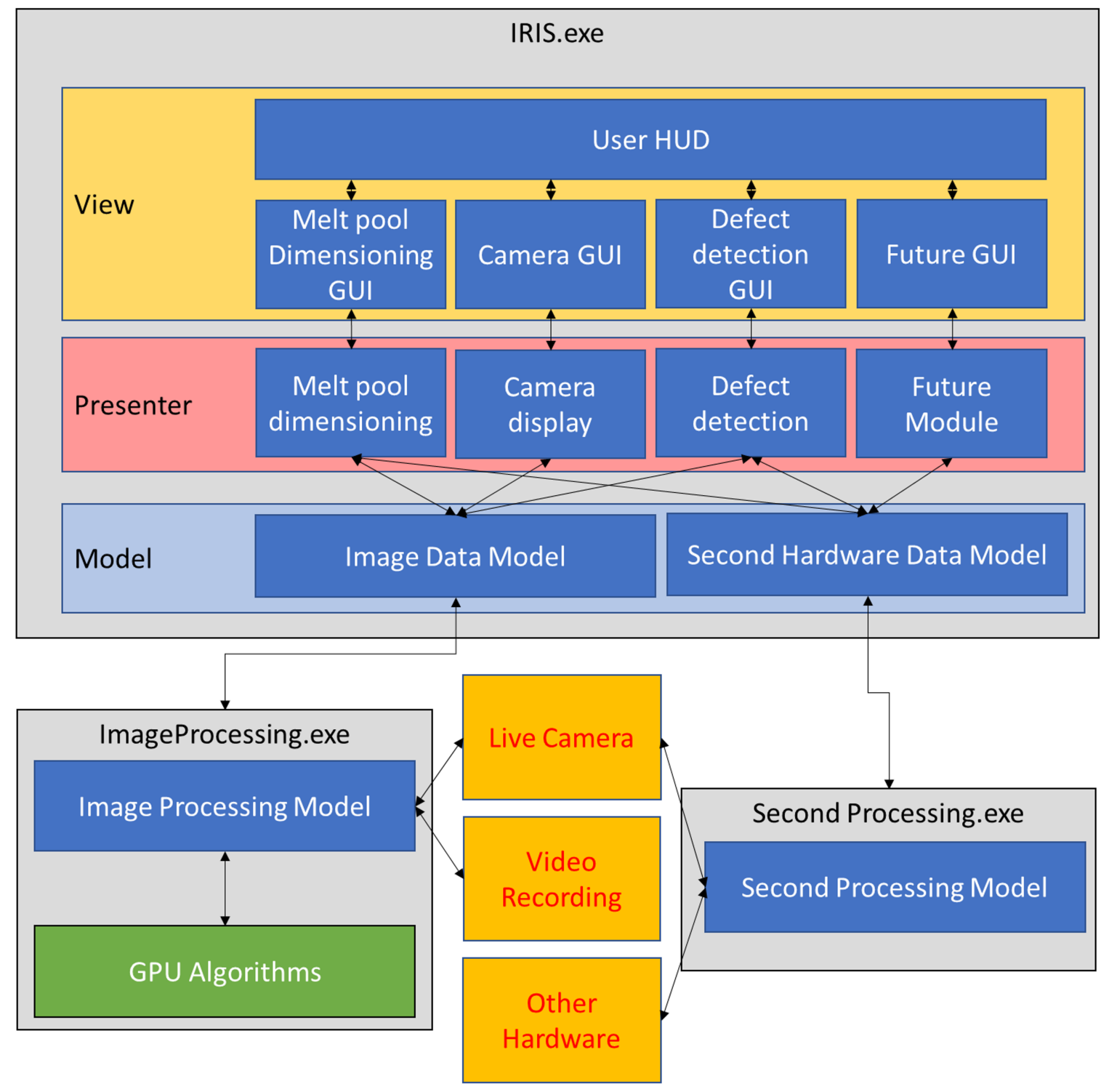

Figure A.2: A schematic diagram representing a future architecture expansion. 


\section{A.3.5 Initial System Modules}

This design encapsulates all features that should be delivered at the end of this research. Whilst all of the system requirements are ideal, carrying all of these forward in the space of a $\mathrm{PhD}$ study is somewhat difficult. Whilst these concepts should be considered in the design now to allow for future implementation, time restrictions dictate that only the most important concepts are incorporated into the initial design. The two core modules here are the camera display and melt pool dimensioning.

\section{A.3.5.1 Camera Display Module}

The camera display is possibly the most fundamental module in the designed system. The first and foremost functionality that the system should have is to display a clear image of the melt pool and allow for in-situ changes to the camera settings. This will grant the user a better understanding of the AM process and allow them to subjectively view the melt pool. Experienced DED technicians can use the camera to monitor discrepancies and drastic melt pool changes, using the HUD to spot errors. Tracking the melt pools behaviour through the AM process results in the user understanding key events that occur in the image feed, which can subsequently be used to develop advanced melt pool image processing algorithms. The algorithms developed and tested in Chapter 4 were created by initially watching the melt pool image feed in this manner.

\section{A.3.5.2 Melt Pool Dimensioning Module}

The second deliverable module from this system should be one that allows for in-situ melt pool measuring that can be displayed to the user. Image processing algorithms developed should be implemented in real time on a live melt pool image feed to allow the user to view melt pool data (in-situ) and store it.

Viewing the information live will again allow the user to subjectively view signal patterns which can subsequently lead to both a better understanding of the melt pool and future algorithm development. Developments are more easily made when there is solid signal familiarity. 


\section{A.4 Algorithm Optimisation}

One of the requirements highlighted in Section A.2, to be incorporated into the original software, was optimum processing speed. At this present moment, the current online processing speed is limited to that of the cameras acquisition rate (currently around $40 \mathrm{fps}$ ). Future work would likely lead to the acquisition of a camera with drastically increased frame rates, which could result in a bottleneck occurring with image processing speed. Post-process image processing is not dependent on camera frame rates, and increasing image processing speed will directly influence the processing speed of post-process video analysis. Image processing on both live and recorded videos would benefit from increasing the performance capabilities of the newly developed image processing technique.

This section of work will now focus on optimising the algorithms developed in Chapter 4. Algorithm optimisation can be performed in many different ways, and this appendix explores optimising the image processing technique using two methods. The directional emittance algorithm is further developed in a $\mathrm{C}++$ environment. Two different algorithms are developed, one using the CPU as a processor and another using a GPU. Both are compared to one another to discover if GPU parallelisation can yield a performance increase. The CPU code is exclusively written in $\mathrm{C}++$ and utilises the OpenCV library for some core image processing functions. The GPU code is exclusively written in $\mathrm{C}++$ and utilises both the OpenCV library for some core image processing functions, and CUDA/C++ extensions. These extensions are used to perform memory allocations, transfer data and launch kernels to perform image processing tasks on the GPU.

\section{A.4.1 C ++ Development}

The first algorithm that was developed after the original prototype was created in $\mathrm{C}++$ using only the $\mathrm{CPU}$ as a processing unit. $\mathrm{C}++$ was chosen as the first language to optimise the original prototype due to its array of benefits. This section details the core language benefits as well as the added OpenCV library used before detailing the performance enhancing methods employed. 


\section{A.4.1.1 Core Language Benefits}

$\mathrm{C}++$ was built upon the foundation of $\mathrm{C}$ by adding features into the language such as object-oriented programming. Though features were added to the language, the core concepts of $\mathrm{C}$ were not abandoned [95]. The $\mathrm{C}++$ programming language is a mid-level programming language that combines features of both low and high level languages. It is a structured language that allows the users to easily break down large blocks of code into more manageable blocks (often functions), making it suited for the implementation of the directional emittance technique. The stages of the new image processing technique are highlighted again:

1. Acquire frame.

2. Convert frame to single channel (only required for video recordings).

3. Circular region of interest.

4. Frame averaging technique.

5. Centre of mass calculation.

6. Extract sector.

7. Smooth sector.

8. Calculate upper and lower thresholds.

9. Differentiate sector (delta sector).

10. Crop delta sector.

11. Calculate north location.

12. Calculate south location.

$\mathrm{C}++$ is a highly portable language meaning that if commercialisation of this software were to happen in the future, the algorithm could be recompiled to run on UNIX, Linux and Windows operating 
systems as well as Intel x32, x64 and ARM based architectures. The language also has an abundance of libraries, which provide support and functionality. External open libraries such as OpenCV can easily be incorporated into $\mathrm{C}++$ languages to allow for easy use of already well established image processing techniques.

All these aspects help when writing a program, but the core reason for using $\mathrm{C}++$ as the programming language of choice is because of its memory management control. $\mathrm{C}++$ allows the user complete control of memory management allowing for the allocation of memory prior to the execution of certain tasks. This means that the required memory for the image processing technique just described can be allocated once at the start of the image processing technique, and reused for every processed frame. $\mathrm{C}++$ can pass large sections of memory (namely the frames) by reference, meaning that pointers can simply be passed around as opposed to large blocks of data. Finally, when $\mathrm{C}++$ is compiled it is turned into machine language that can operate directly with the computer's hardware components. There is no need for a virtual machine to run this code, meaning there is no time spent translating higher level language blocks of code into machine code.

\section{A.4.1.2 OpenCV Library}

The OpenCV library has been invaluable in the optimisation of the new image processing technique. The OpenCV library is a library that has an array of computer vision functions that aid developers in developing $\mathrm{C}++$ code. OpenCV is free for both academic and commercial use and has Java, $\mathrm{C}++$ and Python interfaces, supporting Windows, Linux, OS, iOS and Android [96]. It is designed for real time applications and is written in optimised $\mathrm{C} / \mathrm{C}++$. The library utilises multi-core processing and can be used with OpenCL for hardware acceleration [96].

The library contains many functions that can be used for image processing, but one of the most fundamental components used in this application is its ability to open both cameras and video files. The OpenCV library contains a VideoCapture object that has the basic functionality of opening videos and camera feeds and extracting individual frames. Individual frames are stored in Mat objects which can be manipulated in a variety of different ways. Mat objects can have their channels manipulated, and have certain regions cropped/masked. OpenCV can also display images to the user and can draw 
shapes onto images to display calculated results for development/display purposes.

\section{A.4.1.3 Performance Enhancement Methods}

Development within a $\mathrm{C}++$ environment for this given application without performance enhancing methods will likely yield a speed increase instantly. Though this is the case, certain parts of the code were optimised so that minimal calculations are carried out for each individual frame. Memory management in $\mathrm{C}++$ allows the user to assign blocks of memory and delete them when they no longer have use. Using this technique, all memory is allocated when the algorithm first starts. Memory for all frames, arrays and individual values are assigned before the algorithm is performed on the first frame. This memory allocation means that other processes running on the operating system cannot interfere with memory, and that memory will not be temporarily deleted to allow for the execution of other tasks. The frames were stored in dynamic arrays with the data type of unsigned short. Dynamic arrays are necessary for the given application, as the future software design should allow the user to be able to change the region of interest, thus changing the size of the array. The image data is 8-bit, but unsigned short data types are used as image processing step four requires the frames to be added together before division. Frames are averaged across a window of seven frames, meaning that the data type must be able to store up to seven times the maximum pixel value $(7 \times 255=1785)$.

\section{A.4.2 CUDA $/ \mathrm{C}++$ Development}

GPGPU is a technique that uses GPUs to manipulate data and perform calculations that traditionally would have been performed using a CPU. GPUs were originally designed for the gaming industry, and the purpose of the hardware is to perform thousands of tiny calculations as quickly as possible. In video games, worlds are often created by small triangular shapes, and the graphics cards are required to rapidly translate these triangles and render textures/patterns to surfaces. For this reason the architecture design is optimised for a large magnitude of small calculations [97].

NVIDIA and AMD became the two main GPU manufacturers and NVIDIA soon became the most dominant GPU manufacturer. Their market shares in 2017 were worth over double that of AMD [98]. Their dominance in the industry has led them to be the top manufacturer of desktop GPUs 
for several years. Although NVIDIA saw great success as a GPU designer and manufacturer for the gaming industry, they saw further use of their hardware that extended use into the fields of engineering and science. NVIDIA started to create Compute Unified Device Architecture (CUDA) enabled GPUs that allowed users to program code that would traditionally be run on a CPU, to run on GPUs with increased performance. The CUDA platform can be used with the $\mathrm{C}++$ programming language by providing a software layer that gives direct access to the GPU's virtual instruction set. This allows users to transfer data from RAM (Host), to the GPU's global memory (Device) so that kernels can be launched to perform parallel computations [97].

Computing on the GPU can provide performance acceleration for different processes. NVIDIA's website uses examples such as data science, computational fluid dynamics, numerical analytics, computational finance and image \& computer vision. Parallel computational techniques can be used to increase performance on these calculations and the website boasts the use of its technique in many scientific papers [97].

Due to its successful history of improving performance on image processing techniques, it was decided that this avenue would be explored to distinguish if GPGPU techniques could be used to accelerate the directional emittance image processing technique. GPGPU techniques were implemented on all possible parts of the new directional emittance algorithm, and execution was timed to compare performance.

\section{A.4.3 Experiment - Speed Comparison}

The directional emittance algorithm was written in the $\mathrm{C}++$ programming language using the OpenCV library for a variety of functions. The original MATLAB code was converted to $\mathrm{C}++$ by using the OpenCV library. The OpenCV library provided functions that could handle both live camera feeds and video inputs, as well as providing a variety of already coded image processing functions. OpenCV provided Mat objects that could easily hold images for subsequent manipulation. The library provided a method (cvtColor) that was able to change the default three channel image input into a single 8-bit grey scale image. OpenCV also provided Mask and circle methods, that allowed for the circular ROI to be cast over the original image. 
The directional emittance algorithm was then written using CUDA/C++. The CUDA library provided an array of functions that allowed data to be transferred from host memory to device memory. The function cudaMalloc allows the user to allocate memory on the GPU. The function cudaMemset allows the user to set blocks of memory on the GPU to a set value. cudaMemcpy allows the user to copy memory from the host to the device, with cudaMemcyAsync performing the same function, but allowing for subsequent code to run in parallel to performing device synchronisation. cudaDeviceSynchronize allows the user to synchronise the device. These core functions are used to deal with all data transfers and device synchronisation.

Once data is on the device, kernels can be launched to manipulate data in a parallel fashion. Kernels are written as simple blocks of code, and are launched using triple chevrons in a pattern governed by the launch configuration $(<<<$ gridSize, blockSize $>>>)$. The block size that is launched corresponds to the number of threads launched for a given calculation. The total number of threads that can be launched on current NVIDIA cuda enabled GPUs is 1024. This number is reduced to 512 for some older devices. The grid size then corresponds to the total number of blocks launched. The total number of grids that can be launched is displayed in Figure A.3. The GPU used to increase the performance of the directional emittance algorithm was the GeForce GTX 980M. 


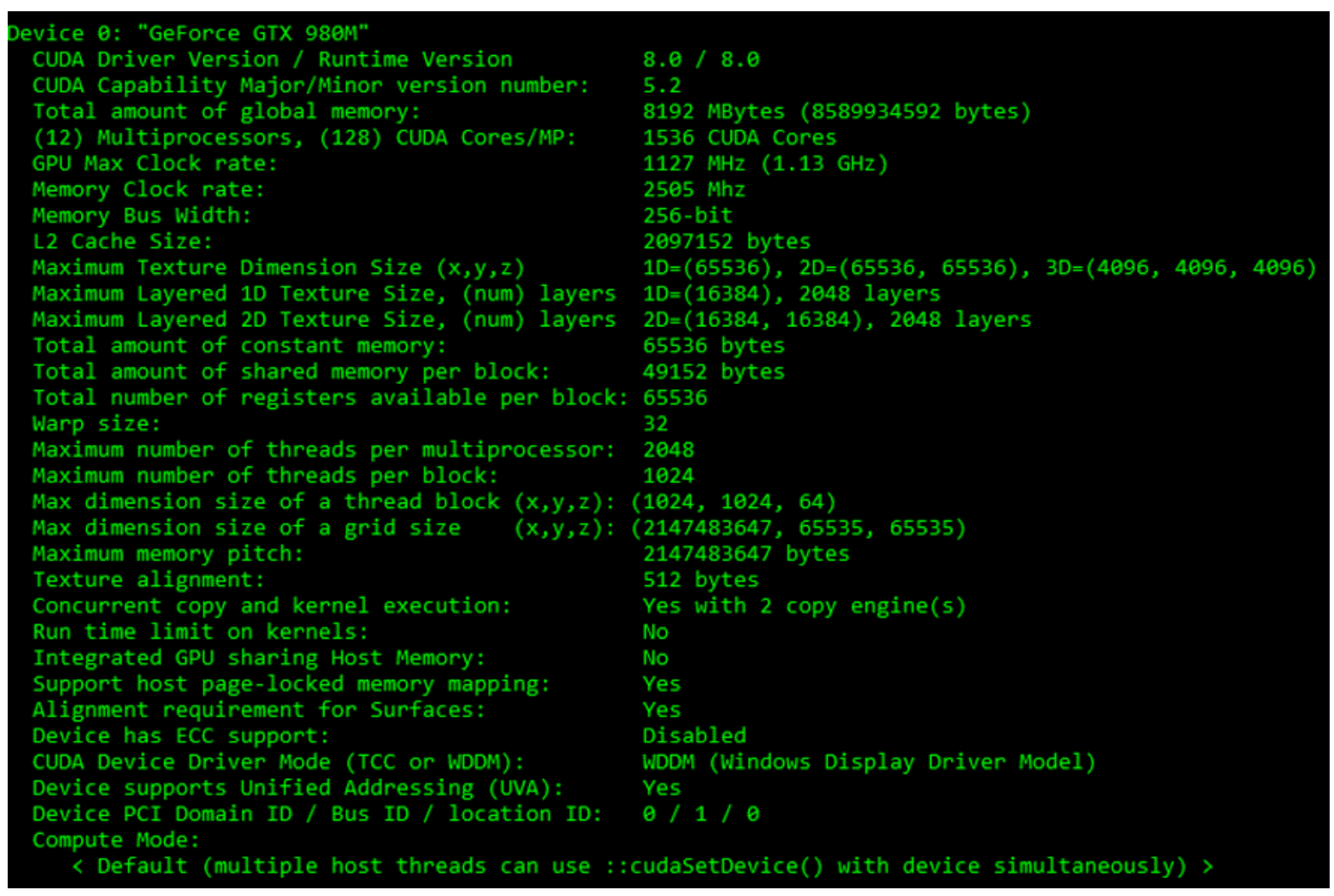

Figure A.3: Information about the GPU used to develop the new image processing algorithm.

\section{A.4.4 Timing Results}

Algorithm timings were performed using the high resolution clock provided in the standard $\mathrm{C}++$ thread library. The high resolution clock class provides access to the current time point and is the clock with the highest precision provided by the standard $\mathrm{C}++$ library. The class allows the user to create time points, and differences between time points can be calculated to determine the duration of computational tasks. The first time point was created before any processing on the first frame was conducted. The second time point was taken once all the frames in the given video were calculated. The total time it took to run the image processing algorithm over the whole video was then divided by the total number of frames in the video (4239). This calculated the average time it took to run 
the directional emittance algorithm on a singular frame for both the $\mathrm{C}++$ and the CUDA/C ++ code. The executable file created was ran multiple times until timing results stabilised. The average time for a single frame to be executed was then recorded for both the developed algorithms. The average time that it took to process frames using the $\mathrm{C}++$ and CUDA/C++ algorithms are displayed in Table A.1.

Table A.1: The average time taken to execute the image process algorithm using CPU and GPU techniques with their corresponding frame rates.

\begin{tabular}{ccc} 
Timing & $\mathrm{C}++$ Algorithm & CUDA $/ \mathrm{C}++$ Algorithm \\
\hline Average Frame Time & $3455 \mu \mathrm{s}$ & $3326 \mu \mathrm{s}$ \\
Frame Rate & $289 \mathrm{fps}$ & $300 \mathrm{fps}$
\end{tabular}

The results display that the CUDA/C ++ implementation of the directional emittance algorithm yielded an increase in performance over the original $\mathrm{C}++$ algorithm. The CUDA library was utilised, and parallel image processing techniques were able to increase the processing speed of the executed algorithm. The CUDA/C++ parallel technique allowed for the frame rate to increase by $11 \mathrm{fps}$. The percentage increase in performance was around $4 \%$ when using the GPU for acceleration of certain image processing functions.

The results showed that there is an advantage of using the CUDA/C++ algorithm instead of the $\mathrm{C}++$ algorithm that runs solely on the CPU. The difference in performance between the two algorithms however is slim. Whilst the CUDA/C++ algorithm showed a performance increase, the advantage of using the CUDA/C++ algorithm was comparability small. NVIDIA quote that the CUDA techniques can be used in many different applications for process acceleration, and although this was true in the case of the directional emittance algorithm, the difference between the CPU and GPU calculations were marginal. This discussion section will give a more qualitative analysis of how the GPU algorithms were implemented, and what implementations saw a speed increase/decrease. This section will walk through the algorithm explanation list explained in Section A.4.1.1. 


\section{A.4.4.1 Frame Acquisition}

The first step in the algorithm is to acquire a frame from the videoCapture object. The videoCapture object is an OpenCV object that can be used to interact with either cameras or recorded video files. This step was identical in both the $\mathrm{C}++$ and CUDA/C ++ versions of the algorithm and CUDA could not be used for process acceleration.

\section{A.4.4.2 Channel Reduction}

The second step in the algorithm was to convert the acquired frame from its default 3-channel input into a single channel 8-bit image. Again, this functionality was performed by an OpenCV function (cvtColor) and CUDA techniques could not be used to grant a performance increase.

\section{A.4.4.3 Circular ROI}

The circular ROI function performed in step 3 was the first function that utilised the CUDA architecture for a performance increase. The ROI function crops parts of the image that are not needed, and subsequently changes regions of the image black to remove the reflected light from the nozzle. This image processing technique was originally performed in the $\mathrm{C}++$ algorithm by creating a rectangle with the desired area and location to extract a portion of the original image. The OpenCV Region function was then used to extract the ROI of the original frame, into a smaller frame for a performance increase. Another Mat object was created (using Mask) with the same dimensions as the new ROI. The OpenCV circle function was used to draw a circle with saturated pixels inside of it and pixels with a value of -1 outside of it. The ROI frame and the mask frame were then combined using the "\&" operator. This resulted in an image that had a reduced size and no reflection radiation from the nozzle.

The CUDA/C++ algorithm utilised the architecture of the GPU to grant a speed performance on this algorithm. Before the start of the first algorithm, a mask was created on the host that consisted of a 401 by 401 array of ones and zeros. The array had ones in the centre of the image in the geometry of a circle, and zeros at the edge of the image where the circle ended. The mask was transferred in the 
GPU set-up period, and stayed on the GPUs memory, being reused for every processed frame. Once the original frame had been copied to the GPU, the ROI kernel was launched to perform the standard ROI functionality. The CUDA/C++ ROI function used a single kernel launch to produce the final circular ROI image. The kernel's input parameters included a memory location for the original image, the ROI mask and the output image where the result would be stored. The kernel also uses the width of the desired ROI image, an X offset and a Y offset. The offsets dictate where the ROI starts. The width of the ROI dictates how large the resulting image would be. The kernel was launched using blocks of 1024 threads in a $32 \times 32$ thread configuration. The amount of blocks (grid size) in both the $\mathrm{X}$ and $\mathrm{Y}$ directions were enough to cover the entire resultant image. This is explained in Equation 17.

$$
G_{x, y}=\frac{L_{x, y}-1}{B_{x, y}+1}
$$

Where $G$ is the grid size in either the $x$ or $y$ direction. $L$ is the length of the image in the $x$ or $y$ direction and $B$ is the block size in the $x$ or $y$ direction.

The kernel was launched with the above configuration, and $x$ and $y$ values were assigned to each thread using both Equations 18 and 19.

$$
\begin{aligned}
& x=\left(b_{i d x . x} \times b_{\text {dim. } . x}\right)+t_{i d x . x} \\
& y=\left(b_{i d x . y} \times b_{d i m . y}\right)+t_{i d x . y}
\end{aligned}
$$

Where $b_{i d x}$ is the block index number in the given $\mathrm{X} / \mathrm{Y}$ direction, $b_{d i m}$ is the block dimension in the given $\mathrm{X} / \mathrm{Y}$ direction, and $t_{i d x}$ is the thread index number in the given $\mathrm{X} / \mathrm{Y}$ direction.

Using the launch configuration above and $x$ and $y$ values for the current thread, each thread first went through the following statement to establish whether the executing thread was required for the 
ROI calculation.

$$
\text { if }(x<w \& \& y<w)
$$

Where $w$ corresponds to the width (or height due to the image being square) of the image. Each thread that passed this statement then performed the following calculation.

$$
R_{x, y}=O_{x+x_{\text {offset }}, y+y_{\text {offset }}} \times M_{x, y}
$$

Where $R$ is the resultant image at the given $\mathrm{X}, \mathrm{Y}$ location, $O$ is the original image at the given $\mathrm{X}, \mathrm{Y}$ location, $x_{\text {offset }} / y_{\text {offset }}$ are the offset values, and $M$ is the mask value at the given X,Y location.

Performing this calculation in the parallel fashion allowed for a performance increase when comparing the CUDA/C++ algorithm with the $\mathrm{C}++$ algorithm.

\section{A.4.4.4 Frame Averaging}

The frame averaging function was another function that benefited from the CUDA/C++ parallel implementation. The frame averaging technique used on the $\mathrm{C}++\mathrm{CPU}$ implementation worked using a series of zeroing, memory copying, adding and dividing functions. A detailed analysis of how this algorithm is designed is described in Section 4.2.1. Before frame manipulation was carried out on the CPU the Mat objects were converted into unsigned short arrays to allow for quicker processing. To average the frames on the CPU, the current input frame was copied to the buffer. This was done using the standard $\mathrm{C}++$ memcpy function. All available frames in the buffer were then added to an unsigned short array containing the cumulative values. The buffer had to be a 16bit unsigned short data type to allow for the summation of multiple 8bit values. Each pixel in the array was then subsequently divided by the total number of frames added from the buffer.

The CUDA/C++ implementation of this function uses the same method of zeroing images, copying memory, adding images together and dividing them, but uses kernels to perform these calculations in a parallel fashion. 
The first step in the CUDA/C++ method is to zero the memory of the average image so that previous images do not interfere with the image to be calculated. This is done using the cudaMemset function on each iteration, and setting all values in the image to zero.

The next step is to then transfer the image to the relevant location in the buffer. The buffer location is calculated using a counter that is reset after the position in the buffer reaches the end. The image is transferred in memory using a kernel with the same launch configuration that was used for the circular ROI function. The block size was $32 \times 32$ and the grid size was calculated using Equation 17. The kernel is launched with this configuration and each thread is assigned an $x$ and $y$ value using Equations 18 and 19.

The kernel's input parameters are the memory address of the input image, the memory address of the desired output image and the size of the image in the $\mathrm{X}$ and $\mathrm{Y}$ directions. Once the kernel is launched, each thread will pass through a conditional statement to ensure that only the desired memory addresses are processed. If the current thread passes through that if statement they will undergo Equation 22.

$$
T_{x, y}=R_{x, y}
$$

Where $T$ is the resultant memory address at the desired buffer location for the given $x, y$ location and $I$ is value at the memory address of the of the input image for the given $x, y$ location.

Once the image is transferred to the buffer a second kernel is launched to compile all images in the buffer into a single unsigned short array. The launch configuration for this kernel was performed in one dimension. The block size was $1024 \times 1$ and the grid size was calculated using Equation 23.

$$
G_{x}=\frac{L_{x}-1}{B_{x}+1}
$$

Where $G$ is the grid size. $L$ is the length of the array and $B$ is the block size.

It is to be noted that there was no difference in speed between the two different launch configurations 
used. This kernel could have easily been launched using the previous launch configuration. Once the kernel is launched, each thread is assigned an $x$ value using Equation 18. Each thread passes through a conditional statement to ensure that no thread that exceeds the size of the image is used in the calculation. Each thread then performs the calculation explained in Equation 24.

$$
S_{x}=\sum_{i=1}^{m} T_{i \times w+x}
$$

Where $S$ is the output summed image at the given memory location $x, T$ is the memory address of the buffer image for a given $x$ location, $m$ is the total number of input images from the buffer, $w$ is the size of the images to be added to the summed image and $x$ is the thread index.

The final stage of the frame averaging function then launches a kernel to divide the summed image into the original 8-bit data range. This is done using the same two-dimensional launch configuration that was used in the transfer image kernel. The block size for this launch configuration was $32 \times 32$. The grid size was calculated using Equation 17. The input parameters for the kernel were the memory address of the input summed image $(S)$, the memory address of the output average image, the value for which each memory address (pixel) is to be divided by and the width and height of the image.

Each thread that was launched was assigned an $x$ and $y$ value calculated using Equations 18 and 19. Each thread was passed through a conditional statement to ensure that no threads that exceeded the size of the image were used. All threads that met these conditions were then subjected to Equation 25.

$$
A_{x, y}=\frac{S_{x, y}}{D}
$$

Where $A$ is the memory address of the resultant image for the given $x / y$ location, $I$ is the memory address of the input image for the given $x / y$ location and $D$ is the total number of images that were originally added to the summed image.

Combining all these image processing kernels together resulted in a speed increase in the frame averaging function used in the directional emittance technique. Performing this calculation in the 
parallel fashion allowed for a performance increase when comparing the CUDA/C ++ algorithm with the $\mathrm{C}++$ algorithm.

\section{A.4.4.5 Centre of Mass}

The third function of calculating the COM was performed using both $\mathrm{C}++$ and CUDA/C ++ techniques, but it was found that the $\mathrm{C}++$ technique was far superior in calculating the $\mathrm{COM}$ than the parallel CUDA technique.

The COM calculation is performed by reducing all rows and columns into two one-dimensional arrays. These two arrays are then subjected to a scan algorithm that provides an accumulative summation of all the cells in the calculated reduced arrays. These arrays are then used to calculate the location, in both the $x$ and $y$ directions, at which half of the pixel intensity of the whole image is met. This algorithm is explained in full in Section 4.2.2.

Both reduction and scan techniques have parallel methods that are documented on the NVIDIA website, that aim to speed up processing by executing many calculations simultaneously. Documentation from the NVIDIA website describes in detail how these techniques can be implemented using CUDA/C++ techniques, but also document limitations of the CUDA implementation relative to the size of data used. A section within the referenced document ([99]) shows the speeds of calculations on the CPU as compared with the CUDA implementation on the GPU for different numbers of elements in the summation. It is shown that for small data sizes (less than 1000 data points), the CUDA parallel implementation of the scan algorithm performed its calculations far slower than the regular CPU implementation. This is because CPUs are far superior at linear processing and the amount of parallelisation that can be achieved with such a small number of elements is minimal. For the documented conventional scan technique, the GPU algorithm's performance exceeds the CPU algorithm's performance when over 65,000 elements are used in the summation. The image that is subject to processing in this system is $401 \times 401$ pixels in size, meaning that the GPU implementation of this technique showed poorer performance when compared to the CPU implementation. Due to the data size in the reduction and scan operations, it was therefore decided that these calculations should be performed on the CPU. 


\section{A.4.4.6 Stages 6 - 12}

Stages 6 through to 12 were all performed on the CPU for the same reasons explained for the COM calculation. All these calculations are either single calculations, or calculations/iterations performed on small arrays of 401 pixels or less. For this reason, implementing any of these techniques on the GPU will have a reduced performance when compared with the standard $\mathrm{C}++$ implementation of the code. For this reason, all of these calculations were performed on the $\mathrm{CPU}$ for both the $\mathrm{C}++$ and the CUDA/C++ implementations of the newly developed algorithm.

\section{A.4.4.7 Detailed Timing and Data Transfer Speeds}

The implementation of the algorithm on the GPU showed an improvement over the standard CPU implementation for both the circular ROI and frame averaging functions. Calculating exactly how long each process took to execute however proved difficult due to the synchronisation processes that occur on the GPU. To optimise the processing time, the amount of synchronisation used on the GPU was reduced due to it being an extremely expensive process. The cudaMemcpy function partnered with the cudaDeviceSynchronize function took far longer to execute than the cudaMemcpyAsync function. For this reason, the cudaMemcpyAsync function was used throughout the main processing loop for data transfer purposes. Implementing this function, instead of the standard cudaMemcpy and cudaDeviceSynchronize, led to a performance increase when using the GPU algorithm. Taking out the cudaDeviceSynchronize functions did however mean that the GPU had full control of when it executed certain kernels and performed device synchronisation. This resulted in the timing of individual functions on the GPU being misleading and often incorrect when using the fully optimised algorithm.

To understand how quickly the kernels were being executed on the GPU compared with the CPU, the cudaDeviceSynchronize function was called prior to the execution of both the circular ROI function and frame averaging function on the CUDA/C ++ implementation. This allowed for more accurate and more precise execution timings. The same timers used for timing whole frame executions (as described in Section A.4.4) were used to time the circular ROI and frame averaging functions executing 
on both the GPU and CPU so that direct comparisons could be made. The same video that was used to calculate whole frame execution times was again used to find out the detailed execution times of the circular ROI and frame averaging functions. The results were therefore averaged over 4239 frames. The results comparing the execution times for both functions on the CPU and the GPU are displayed in Table A.2.

Table A.2: Detailed timing results showing the executing times for both the circular ROI and frame averaging functions.

\begin{tabular}{ccc} 
Function & C++ Algorithm & CUDA $/ \mathrm{C}++$ Algorithm \\
\hline Circular ROI & $184 \mu \mathrm{s}$ & $9 \mu \mathrm{s}$ \\
Frame Averaging & $547 \mu \mathrm{s}$ & $18 \mu \mathrm{s}$
\end{tabular}

The results from Table A.2 show a drastic reduction in execution times when using the GPU as opposed to the $\mathrm{CPU}$ for both the circular ROI and frame averaging functions. The circular ROI function performed over twenty times faster on the GPU and the frame averaging function performed over thirty times faster on the GPU. These timing results show that executing certain image processing functions on the GPU can result in a huge reduction in execution times.

Although operating on the GPUs showed such a drastic reduction in execution time when compared to the CPU algorithms, the overall difference between execution times between the algorithms was far less. The time to execute the circular ROI and frame averaging functions on the GPU was $27 \mu s$. The time to execute the circular ROI and frame averaging functions on the CPU was $731 \mu s$. The difference between these two execution timings is over $700 \mu s$ and the difference between the average whole frame execution time calculated in Section A.4.4 is $129 \mu \mathrm{s}$. This difference in time is due to the data transfer operations that move data from the host to the device, combined with the costly cudaDeviceSynchronize function.

To reduce the expensive data transfer rate as much as possible, all data arrays were converted into unsigned char arrays before being transferred to and from the device. This reduced the overall transfer rates as compared to using an unsigned short data type. For all the timing experiments, the unsigned char data type was used to transfer data to and from the device. 


\section{A.4.5 Conclusion}

Implementing the newly developed algorithm onto the GPU using the CUDA/C++ API allowed for an increase in performance. The GPU implementation of the directional emittance algorithm showed a $4 \%$ increase overall when compared with the $\mathrm{C}++\mathrm{CPU}$ algorithm. The circular ROI and frame averaging functions showed a twenty and thirty times performance increase respectively.

Although a performance increase was achieved by using the CUDA/C ++ API, the performance of the CUDA/C ++ implementation was drastically inhibited by the data transfer rates from both the host to device, and the device to the host. This data transfer rate was reduced by converting the arrays to be transferred into an unsigned char data type.

The performance increase achieved from the GPU, although only small, showed that the technique can be used to accelerate the directional emittance algorithm. The small increase in processing performance means that the GPU algorithm will be implemented into the final design of the prototype melt pool monitoring system.

Further work can extend the performance enhancements achieved from the GPU. The data transfer rates showed to be the major bottleneck in using the CUDA/C++ API to provide performance increase, but a huge reduction in frame execution time was achieved once the data was in GPU memory. Further melt pool image processing techniques used on the same image feed could result in a greater difference in performance when comparing the algorithm with a standard CPU implementation. Any subsequent image processing technique that is complemented by the parallel nature of the CUDA/C ++ API can easily be implemented on the GPU without the need to worry about data transfer rates. Even with data transfer rates, the implementation of the two algorithms onto the GPU showed a performance increase. As more image processing algorithms are developed, the use of GPGPU programming as a method to increase processing performance will become more effective. 


\section{A.5 Graphic User Interface Design}

This appendix has witnessed the development and optimisation of a CUDA/C ++ image processing algorithm that can run on a standard laptop GPU. Using a portable GeForce GTX 980M, a frame rate of 300fps was achieved. This method of calculating the melt pool width has a frame rate that greatly exceeds that of the camera that is currently used in the system, allowing for real time melt pool data acquisition. The newly developed GPU algorithm has capabilities that greatly exceed the current hardware and allows for the future expansion of the current DED system. The new GPU algorithm can also be used to process previously recorded data at a much quicker rate for more swift academic studies.

This new GPU algorithm provides a solid model that a front end application can be developed for. This solid foundation can be used with a JavaFX interface to easily display melt pool behaviour to users.

Using the MVP system architecture described in Section A.3.1.1 an application was developed for both industrial and academic use. The developed system used the CUDA/C++ implementation of the directional emittance algorithm with a JavaFX front end to allow for easy use of the academic developments. The IRIS application developed allows the user to open the USB camera and manipulate camera settings such as the exposure time and area of interest. The programs functionality allows users to play and pause live feeds, and view melt pool width calculations live during deposition. Users can also save data to text files. The designed GUI is displayed in Figure A.4. 


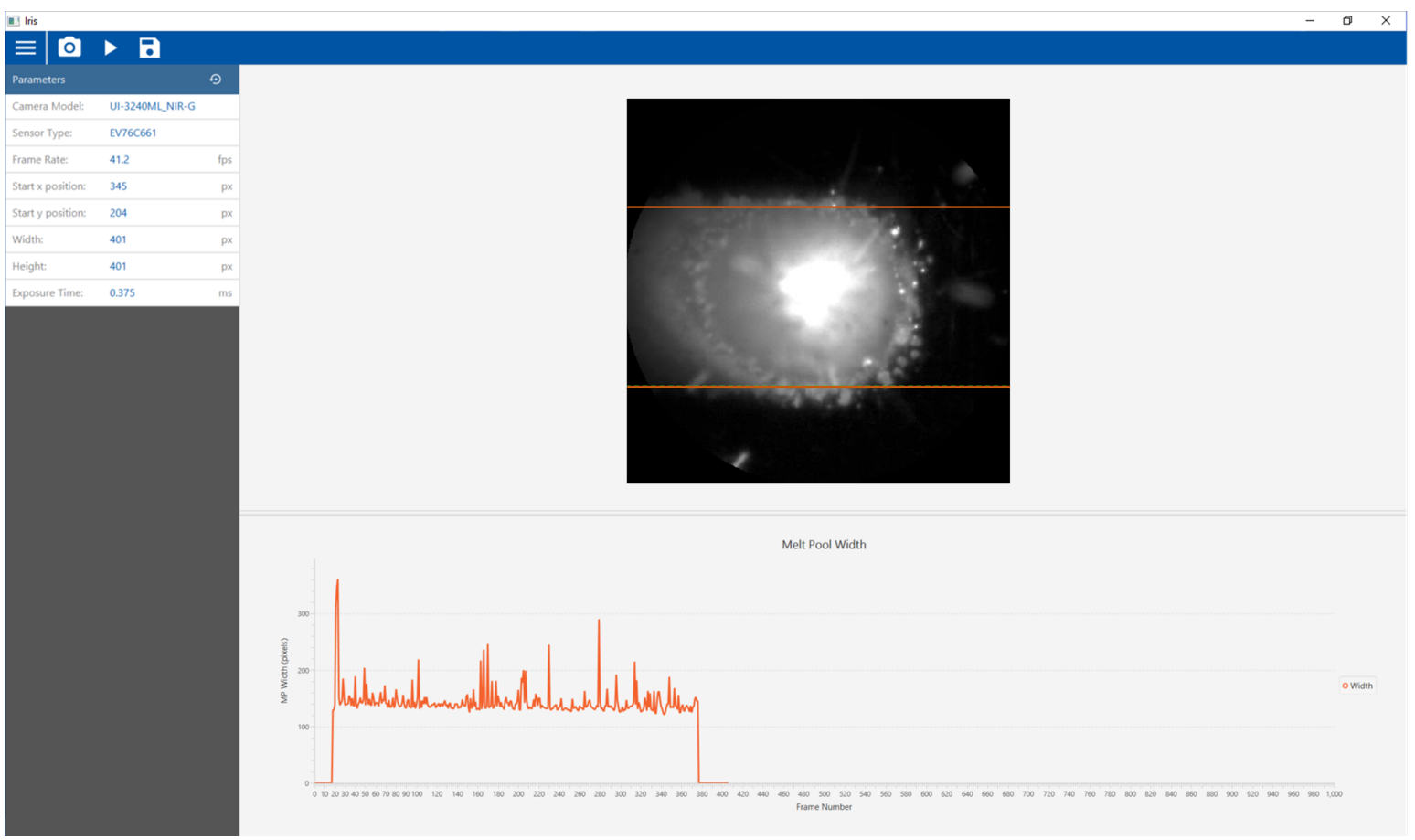

Figure A.4: A screenshot of the final GUI design implemented into TWI's industrial environment.

\section{A.6 Appendix Conclusion}

This Appendix has seen the design of system architecture, optimisation of the directional emittance algorithm and software development for internal use at TWI.

The system architecture design chosen was the MVP model due to its high rating in software development stages. The newly developed melt pool monitoring system is in its infancy and due to the expanding field of AM, the software that houses this newly developed image processing technique should be able to rapidly adapt to changes in the field. New methods of controlling the DED process should easily be incorporated into the software architecture, and the MVP model allows for multiple modules to be developed in conjunction with each other by different developers. This model will allow for more parameter monitoring functions to easily be incorporated into the already existing system.

With the skeleton for the new system designed, the optimisation of the underlying image processing 
function was carried out using GPUs as the main method of data acceleration. The CUDA/C++ API was used to transfer data from the GPU so that kernels could execute parallel versions of the algorithm. Computations were run exclusively on the CPU and were compared with computations that utilised the GPU hardware. It was reported that the GPU hardware offered a $4 \%$ performance increase compared with the exclusive $\mathrm{C}++\mathrm{CPU}$ algorithm.

Although the performance increase of the algorithm was only $4 \%$, the performance increase of the circular ROI and frame averaging functions was twenty and thirty times that of their CPU counterparts. This drastic increase in performance on the GPU was however hindered by the computationally heavy data transfer rates that were required to move image data from host memory to device memory. It was also inhibited by the costly device synchronisation process. Nevertheless, the performance increase resulted in the GPU algorithm being chosen to be incorporated into the system architecture. The performance increase provided by the GPU will likely become more apparent as more image processing techniques are developed for the same image feed.

With the skeleton designed and the directional emittance algorithm optimised, the creation of an inhouse melt pool monitoring system was undertaken. The newly designed melt pool monitoring system is able to measure the melt pool in real time, and provide a live response that the user can monitor. This allows the operator to tailor parameters according to melt pool geometry, providing more control over the DED process. The newly installed camera and melt pool monitoring system also allows the user to easily monitor melt pool fluctuations that often correspond to defaults or discrepancies within the build.

Research from Chapters 3 and 4 have allowed for the development of a full melt pool monitoring system that can be implemented on an array of DED machines. The combination of adaptive exposure times and a new image processing algorithm has allowed for more accurate monitoring of the melt pool in DED processes. The system developed in this appendix has encapsulated these academic advancements into a single system that can be used in practical industrial environments. 
214 